Supporting Information

\title{
Alkynylboration Reaction Leading to Boron-containing $\pi$-Extended cis-Stilbenes as a Highly Tunable Fluorophore
}

\author{
Marina Nogami, ${ }^{+}$Keiichi Hirano ${ }^{*,+}$ Kensuke Morimoto, ${ }^{\dagger}$ Masaru Tanioka, ${ }^{\dagger}$ \\ Kazunori Miyamoto, ${ }^{\dagger}$ Atsuya Muranaka, ${ }^{\dagger}$ and Masanobu Uchiyama ${ }^{*,+,}$,
}

${ }^{+}$Graduate School of Pharmaceutical Sciences, The University of Tokyo, 7-3-1 Hongo, Bunkyo-ku, Tokyo 113-0033, Japan

${ }^{\ddagger}$ Cluster for Pioneering Research (CPR), Advanced Elements Chemistry Laboratory, RIKEN, 2-1 Hirosawa, Wako-shi, Saitama 351-0198, Japan

\section{Table of Contents}

1 General

2-1. Preparation of Alkynamides

2-2. Optimization of Reaction Conditions

S-12

2-3. General Procedure for Alkynylboration of Alkynamides

S-13

2-4. Generality of of $N$-substiuents (Scheme 2)

S-14

2-5. Scope of Acetylenic Terminus (Scheme 3)

S-19

2-6. Transformation of Products (Schemes 4)

$S-26$

2-7. Computational Studies (Figure 4)

S-27

2-8. Electronic Absorption and Fluorescence Spectra (Table 1)

S-29

3 References in Experimental Section 


\section{General}

\section{- Instrumentation.}

NMR spectra were obtained on a Bruker AVANCE III HD 500 spectrometer and a Bruker Ascend 400 spectrometer. Chemical shifts are expressed in $\delta(\mathrm{ppm})$ values, and coupling constants are expressed in hertz $(\mathrm{Hz})$. ${ }^{1} \mathrm{H}$ and ${ }^{13} \mathrm{C} \mathrm{NMR}$ spectra were referenced to tetramethylsilane as an internal standard. The following abbreviations are used: $\mathrm{s}=$ singlet, $\mathrm{d}=$ doublet, $\mathrm{t}=$ triplet, quint $=$ quintet, sep = septet, $\mathrm{m}=$ multiplet, $\mathrm{br} \mathrm{s}=$ broad singlet, and br $\mathrm{d}=$ broad doublet. Melting points were determined with a Yanaco micro melting point apparatus and uncorrected. IR spectra were obtained on a JASCO FT/IR-4700 spectrophotometer. ESI mass spectra were measured on a Bruker micrOTOF-II spectrometer. Electronic absorption spectra were measured using a JASCO V-630 or a JASCO V-670. A n integrating sphere was used to obtain the solid-state absorption spectra and reflectance data were converted to absorbance data according to the Kubelka-Munk equation. Emission spectra were recorded on a JASCO FP-8500 spectrofluorometer. All samples were excited at $300 \mathrm{~nm}$. The absolute fluorescence quantum yield $\left(\Phi_{\mathrm{FL}}\right)$ was measured using an integrating sphere. Yamazen medium pressure liquid chromatography (MPLC) system (EPCLC-W-Prep 2XY A-Type) and JAI Recycling Preparative HPLC (LC-9110 II NEXT, mobile phase: chloroform) were used for purification of products. Data collections for X-ray analysis were performed at $90 \mathrm{~K}$ on a RIGAKU XtaLAB Synergy-S diffractometer.

\section{- Materials.}

Unless otherwise noted, materials were purchased from Wako Pure Chemical Industries, Ltd., Tokyo Chemical Industry Co., Ltd., Sigma-Aldrich Co., LLC., and other commercial suppliers. ${ }^{\mathrm{n}} \mathrm{BuLi}$ in ${ }^{\mathrm{n}}$ hexane was obtained from Kanto Chemical Co., Inc. Alkynylboronate $\mathbf{2 a}$ was purchased from Sigma-Aldrich Co., LLC. (90\% purity). 3-Methoxy-1-propyn-1-ylboronic acid pinacol ester (for 6a), 5-chloro-1-pentynyl-1-boronic acid pinacol ester (for 6b), and 2-((tert-Butyldimethylsilanyl)ethynyl) boronic acid pinacol ester (for 6c) were also purchased from Sigma-Aldrich Co., LLC. Alkynamides $1 \mathbf{a},{ }^{1} \mathbf{1 b},{ }^{1} \mathbf{1 g},{ }^{2} \mathbf{1 h},{ }^{3} \mathbf{1 n},{ }^{4} \mathbf{1 o},{ }^{5} \mathbf{1} \mathbf{p},{ }^{2} \mathbf{4} \mathbf{d}^{1}$ were prepared according to the literatures. $\mathbf{1 j}$ is commercially available from LabNetwork. Anhydrous THF was purchased from Kanto Chemical Co., Inc. All chemicals were of reagent grade and used as received. Air- and moisture-sensitive manipulations were performed with standard Schlenk techniques under argon atmosphere. Medium pressure liquid chromatography was performed using the disposable Hi-flash premium column from Yamazen Co., and preparative thin-layer chromatography was carried out using Merck Silica Gel $60 \mathrm{PF}_{254}$, and thin-layer chromatography was carried out on $0.25 \mathrm{~mm}$ Merck silica gel plates (60F-254). 


\section{Experimental Details}

\section{2-1. Preparation of Alkynamides}

\section{General Protocol A:}

To a solution of the corresponding propynoic acid (11 mmol, 1.1 eq.) in $\mathrm{CH}_{2} \mathrm{Cl}_{2}(20 \mathrm{~mL})$ were added a solution of 4-dimethylaminopyridine ( $1 \mathrm{mmol}, 0.1$ eq.) and dicyclohexylcarbodiimide (DCC; $11 \mathrm{mmol}, 1.1$ eq.) in $\mathrm{CH}_{2} \mathrm{Cl}_{2}(10 \mathrm{~mL}$ ) drop wise at $0{ }^{\circ} \mathrm{C}$, then the mixture was stirred for ca. $10 \mathrm{~min}$. A solution of the corresponding primary amine $(10 \mathrm{mmol}$, 1.0 eq.) in $\mathrm{CH}_{2} \mathrm{Cl}_{2}(10 \mathrm{~mL})$ was added dropwise. The resultant mixture was stirred at room temperature for $16 \mathrm{~h}$. The crude mixture was filtered through a short pad of silica gel and eluted with ether. The filtrate was pre-adsorbed on silica gel and chromatographed with MPLC (eluted typically with a mixture of ${ }^{n}$ hexane and EtOAc). If required, materials were further purified either by recrystallization or with the recycling preparative HPLC prior to use.

\section{General Protocol B:}

Reaction setup was the same as General Protocol A, except for the use of EDCI instead of DCC. Triethylamine (1.2 eq.) was also used. Reaction was quenched with $1 \mathrm{~N} \mathrm{HCl}$ aq., phases were separated and the organic layer was washed with $\mathrm{H} 2 \mathrm{O}$ ( $x_{2}$ ) and brine ( $x_{1}$ ), then dried over $\mathrm{Na}_{2} \mathrm{SO}_{4}$. After filtration, the filtrate was pre-adsorbed on silica gel and purification was conducted in the same manner as General Protocol A.

\section{General Protocol C:}

To solution of the corresponding (2,2-dibromovinyl)arene in THF $(0.32 \mathrm{M})$ was added nBuLi in " hexane (2.1 eq.) at $78{ }^{\circ} \mathrm{C}$ and the resultant solution was stirred for $45 \mathrm{~min}$. Methyl chloroformate (1.2 eq.) was added dropwise at the same temperature before the reaction was allowed to warm to $\mathrm{rt}$ gradually. After being stirred for $16 \mathrm{~h}$ at $\mathrm{rt}$, the reaction was quenched by sat. $\mathrm{NH}_{4} \mathrm{Cl}$ aq. and the product was extracted with EtOAc (x 3). The combined organic phase was dried over $\mathrm{Na}_{2} \mathrm{SO}_{4}$, filtered, and concentrated in vacuo to afford the crude methyl ester.

This material was dissolved in MeOH/THF (1:1, $0.25 \mathrm{M})$ and treated with a solution of $0.73 \mathrm{M} \mathrm{NaOH}$ aq. (1.5 eq.) and stirred until the ester was disappeared by TLC analysis. Organic volatiles were removed in vacuo and the aqueous residue was washed ether (x 1), acidified with $1 \mathrm{~N} \mathrm{HCl}$ aq to $\mathrm{pH} 1$, then extracted with $\mathrm{CHCl} 3(\mathrm{x} 3)$. The combined organic layer was washed with brine ( $\mathrm{x}$ 1), dried over $\mathrm{Na}_{2} \mathrm{SO}_{4}$, filtered, and concentrated in vacuo to afford the crude carboxylic acid. The crude acid was transformed to the corresponding amide following General Protocol B.

\section{General Protocol D:}

To solution of the corresponding alkyne in THF $(0.5 \mathrm{M})$ was added $\mathrm{nBuLi}$ in ${ }^{\mathrm{n}}$ hexane $\left(1.0\right.$ eq.) at $-78^{\circ} \mathrm{C}$ and the resultant solution was stirred at $0{ }^{\circ} \mathrm{C}$ for $30 \mathrm{~min}$. The solution was cooled to $-78{ }^{\circ} \mathrm{C}$ and methyl chloroformate $(1.2$ eq.) was added dropwise at the same temperature before the reaction was allowed to warm to rt gradually. After being stirred for $16 \mathrm{~h}$ at $\mathrm{rt}$, the reaction was quenched by sat. $\mathrm{NH}_{4} \mathrm{Cl}$ aq. and the product was extracted with EtOAc ( $\mathrm{x} 3$ ). The combined organic phase was dried over $\mathrm{Na}_{2} \mathrm{SO}_{4}$, filtered, and concentrated in vacuo to afford the crude methyl ester. Following transformations leading to the corresponding alkynamide is conducted according to General Protocol B.

Most of the amides were obtained as a stereoisomeric mixture and the NMR signals of the minor isomers are expressed by italicized values. 


\section{$N$-isobutyl-3-phenylpropiolamide (1c): General Protocol A}

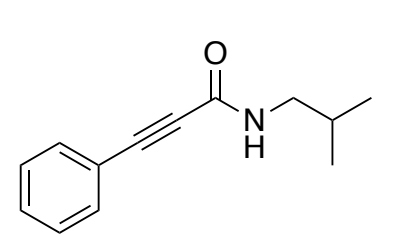

White solid, $76 \%$ (1.52 g) (10.0 mmol scale)

Ratio of streoisometric mixture: $1: 7.0 .{ }^{1} \mathbf{H}$ NMR $\left(400 \mathbf{~ M H z}, \mathbf{C D C l}_{3}\right): \delta 0.96(\mathrm{~d}, J=6.7 \mathrm{~Hz}$, $6 \mathrm{H}), 0.98(\mathrm{t}, J=6.7 \mathrm{~Hz}, 6 \mathrm{H}), 1.85$ (apparent sep, $J=6.7 \mathrm{~Hz}, 1 \mathrm{H}$; minor isomer overlapped), $3.19(\mathrm{t}, J=6.5 \mathrm{~Hz}, 2 \mathrm{H}), 3.31(\mathrm{t}, J=6.7 \mathrm{~Hz}, 2 \mathrm{H}), 6.11(\mathrm{br} \mathrm{s}, 1 \mathrm{H}$; minor isomer overlapped), 7.33-7.44 (m, 3H; minor isomer overlapped), 7.52-7.56 (m, $2 \mathrm{H}$; minor isomer overlapped).

${ }^{13} \mathbf{C}$ NMR (100 MHz, $\mathbf{C D C l}_{3}$ ): $\delta$ 19.8, 20.0, 28.4, 29.5, 47.2, 50.9, 80.8, 83.1, 84.4, 90.8, 120.2 (minor isomer overlapped), 128.4, 128.5, 129.9, 130.2, 132.4 (minor isomer overlapped), 153.5, 156.4. IR (ATR-IR): 3249, 3057, 2959, 2927, 2871, $2217,1626,1541,1490,1466,1443,1388,1369,1289,1219,1158,914,756,689 \mathrm{~cm}^{-1}$. HRMS (ESI): $\mathrm{m} / z$ calculated for $\mathrm{C}_{13} \mathrm{H}_{15} \mathrm{NNaO}^{+}[\mathrm{M}+\mathrm{Na}]^{+}=224.1046$, found: 224.1045 .

\section{N-benzyl-3-phenylpropiolamide (1d): General Protocol A}<smiles>O=C(C#Cc1ccccc1)NCc1ccccc1</smiles>

White solid, $76 \%$ yield $(793.6 \mathrm{mg})(5.0 \mathrm{mmol} \mathrm{scale})$

Spectral data are in agreement with the literature. ${ }^{6}$

$\mathrm{N}$-isopropyl-3-phenylpropiolamide (1e): General Protocol A

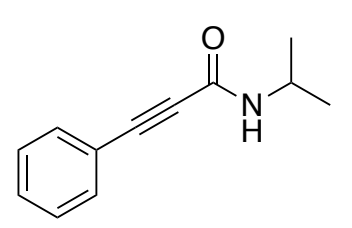

Yellowish oil, 37\% yield $(644.6 \mathrm{mg})(10 \mathrm{mmol})$

Spectral data are in agreement with the literature. ${ }^{7}$

N-cyclopropyl-3-phenylpropiolamide (1f): General Protocol B

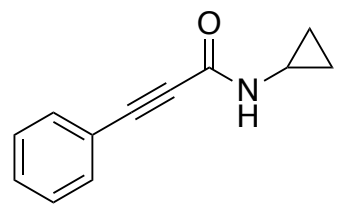

Off-white solid, $24 \%$ yield $(220.9 \mathrm{mg})(5 \mathrm{mmol})$

Ratio of streoisometric mixture: 1 : 4.4. ${ }^{1} \mathbf{H}$ NMR (400 $\left.\mathbf{~ M H z}, \mathbf{C D C l}_{3}\right): \delta 0.60-0.64(\mathrm{~m}, 2 \mathrm{H})$, 0.72-0.76 (m, 2H), 0.81-0.91 (m, 2H; minor isomer overlapped), 2.77-2.87 (m, $1 \mathrm{H}$; minor isomer overlapped), 6.29 (br s, $1 \mathrm{H}$; minor isomer overlapped), 7.32-7.44 (m, $3 \mathrm{H}$; minor isomer overlapped), 7.50-7.52 (m, 2H), 7.56-7.58 (m, 2H). ${ }^{13} \mathbf{C}$ NMR (100 $\left.\mathbf{~ M H z , ~} \mathbf{C D C l}_{3}\right): \delta$ 6.7, 7.9, 22.8, 24.2, 81.9, 83.0, 84.7, 91.3, 120.1, 120.4, 128.4, 128.5, 130.0, 130.2, 132.4, 132.5, 154.7, 158.2. IR (ATR-IR): 3233, 3015, 2220, 1624, 1532, 1303, 1232, 1201, 992, 913, 756, 688, $602 \mathrm{~cm}^{-1}$. HRMS (ESI): $m / z$ calculated for $\mathrm{C}_{12} \mathrm{H}_{11} \mathrm{NNaO}^{+}[\mathrm{M}+\mathrm{Na}]^{+}=$ 208.0733, found: 208.0743 . 
<smiles>O=C(C#Cc1ccccc1)NCCO[Na]</smiles>

White solid, $56 \%$ yield $(516.1 \mathrm{mg})(2.67 \mathrm{mmol} \mathrm{scale})$

Ratio of streoisometric mixture: 1 : 8.6. ${ }^{1} \mathbf{H}$ NMR (400 $\left.\mathbf{~ M H z}, \mathbf{C D C l}_{3}\right)$ : $\delta$ 1.05-1.17 (m, $21 \mathrm{H}$; minor isomer overlapped), $3.50(\mathrm{q}, J=5.3 \mathrm{~Hz}, 2 \mathrm{H}), 3.64(\mathrm{q}, J=5.3 \mathrm{~Hz}, 2 \mathrm{H}), 3.82$ $(\mathrm{t}, J=5.3 \mathrm{~Hz}, 2 \mathrm{H}), 3.84(\mathrm{t}, J=5.3 \mathrm{~Hz}, 2 \mathrm{H}), 6.12(\mathrm{br} \mathrm{s}, 1 \mathrm{H}), 6.37(\mathrm{br} \mathrm{s}, 1 \mathrm{H}), 7.33-7.43$

(m, 3H; minor isomer overlapped), 7.51-7.56 (m, 2H; minor isomer overlapped). ${ }^{13} \mathbf{C}$

NMR (100 MHz, $\mathbf{C D C l}_{3}$ ): $\delta 11.78,11.82,17.9$ (minor isomer overlapped), 42.0, 45.8, 61.8, 62.6, 80.8, 82.9, 84.6, 90.6, 120.18, 120.23, 128.4, 128.5, 129.9, 130.2, 132.4 (minor isomer overlapped), 153.4, 155.8. IR (ATR-IR): 3266, 3061, 2942, 2891, 2865, 2222, 1634, 1541, 1459, 1364, 1300, 1219, 1105, 1070, 915, 882, 756, 687, $658 \mathrm{~cm}^{-1}$. HRMS (ESI): $m / z$ calculated for $\mathrm{C}_{20} \mathrm{H}_{31} \mathrm{NNaO}_{2} \mathrm{Si}^{+}[\mathrm{M}+\mathrm{Na}]^{+}=368.2016$, found: 368.2017 .

2-((triisopropylsilyl)oxy)ethan-1-amine was prepared according to the patent. ${ }^{8}$

\section{3-phenyl-N-(prop-2-yn-1-yl)propiolamide (1k): General Protocol B}

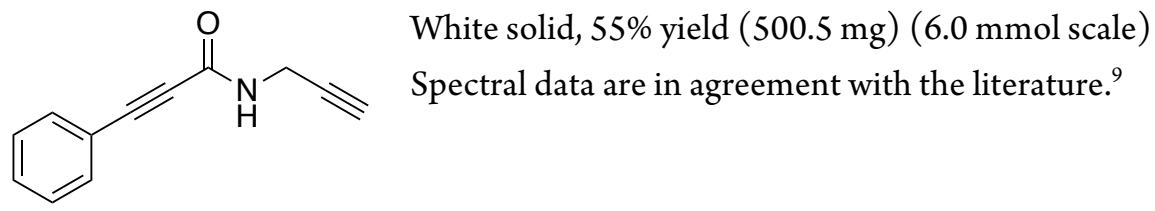

\section{(R)-3-phenyl-N-(1-phenylethyl)propiolamide (11): General Protocol B}

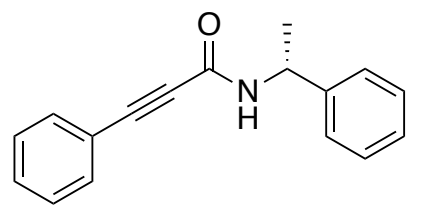

Off-white solid, 51\% yield $(639.4 \mathrm{mg})(5.0 \mathrm{mmol})$

Spectral data are in agreement with the literature. ${ }^{10}$

\section{$N$-methoxy-3-phenylpropiolamide (1m): General Protocol B}

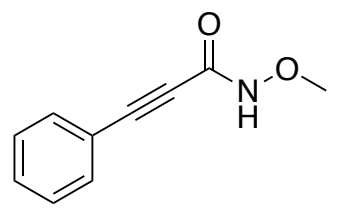

Slightly yellow oil, $76 \%$ yield $(916.2 \mathrm{mg})(7.0 \mathrm{mmol} \mathrm{scale})$

${ }^{1}$ H NMR (400 MHz, CDCl 3 ): $\delta 3.86$ (s, 3H), 7.36 (br t, $\left.J=7.3 \mathrm{~Hz}, 2 \mathrm{H}\right), 7.44$ (br t, $J=7.3 \mathrm{~Hz}$, 1H), 7.53 (br d, $J=6.4 \mathrm{~Hz}, 2 \mathrm{H}$ ), 8.95 (br s, $1 \mathrm{H}$ ). ${ }^{13} \mathbf{C}$ NMR (100 MHz, $\mathbf{C D C l}_{3}$ ): $\delta 64.8,87.8$, 119.6, 128.5, 130.5, 132.6, 152.0. IR (ATR-IR): 3166, 2972, 2938, 2817, 2213, 1636, 1507,

1491, 1443, 1304, 1217, 1064, 971, 934, 914, 783, 756, $688 \mathrm{~cm}^{-1}$. HRMS (ESI): $m / z$ calculated for $\mathrm{C}_{10} \mathrm{H}_{9} \mathrm{NNaO}_{2}{ }^{+}[\mathrm{M}+\mathrm{Na}]^{+}=198.0525$, found: 198.0528 .

\section{$N$-mesityl-3-phenylpropiolamide (1q): General Protocol A}<smiles>Cc1cc(C)c(NC(=O)C#Cc2ccccc2)c(C)c1</smiles>

Off-white solid, $26 \%$ yield $(340.1 \mathrm{mg})(5.5 \mathrm{mmol} \mathrm{scale})$

Ratio of streoisometric mixture: $1: 2.4 .{ }^{1} \mathbf{H}$ NMR $\left(400 \mathbf{M H z}, \mathbf{C D C l}_{3}\right): \delta 2.26(\mathrm{~s}, 6 \mathrm{H}), 2.27$ (s, 6H), $2.30(\mathrm{~s}, 3 \mathrm{H}), 2.32(\mathrm{~s}, 3 \mathrm{H}), 6.91(\mathrm{~s}, 2 \mathrm{H}), 6.95(\mathrm{~s}, 2 \mathrm{H}), 7.13-7.16(\mathrm{~m}, 3 \mathrm{H}), 7.21(\mathrm{br}$ $\mathrm{s}, 1 \mathrm{H}), 7.23-7.26(\mathrm{~m}, 2 \mathrm{H}), 7.32-7.35(\mathrm{~m}, 1 \mathrm{H}), 7.35-7.39(\mathrm{~m}, 2 \mathrm{H}), 7.41-7.46(\mathrm{~m}, 1 \mathrm{H})$, 7.60-7.57 (m, 2H). ${ }^{13} \mathbf{C}$ NMR (100 MHz, $\left.\mathbf{C D C l}_{3}\right): \delta 18.3,18.5,20.9,21.0,81.2,83.1,85.2$, 
89.7, 120.06, 120.09, 128.3, 128.5, 128.9, 129.0, 129.9, 130.1 (minor isomer overlapped), 131.3, 132.6, 132.7, 135.3, 136.8, 137.6, 138.0, 151.9, 156.6. IR (ATR-IR): 3209, 2971, 2921, 2858, 2214, 1607, 1508, 1442, 1375, 1311, 1298, 1235 , 1193, 1027, 969, 912, 842, 757, 732, $689 \mathrm{~cm}^{-1}$. HRMS (ESI): $\mathrm{m} / z$ calculated for $\mathrm{C}_{18} \mathrm{H}_{17} \mathrm{NNaO}^{+}[\mathrm{M}+\mathrm{Na}]^{+}=286.1202$, found: 286.1212 .

\section{N-ethyl-3-(naphthalen-1-yl)propiolamide (4a): General Protocol C}

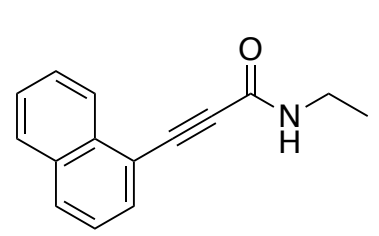

White solid, $43 \%$ yield over 3 steps ( $709.9 \mathrm{mg})$ ( $7.4 \mathrm{mmol} \mathrm{scale})$

Ratio of streoisometric mixture: $1: 7.9 .{ }^{1} \mathbf{H}$ NMR (400 $\left.\mathbf{~ M H z}, \mathbf{C D C l}_{3}\right): \delta 1.23(\mathrm{t}, J=7.3 \mathrm{~Hz}$, $3 \mathrm{H}), 1.28(\mathrm{t}, J=7.2 \mathrm{~Hz}, 3 \mathrm{H}), 3.44(\mathrm{dq}, J=1.4,7.3 \mathrm{~Hz}, 2 \mathrm{H}$ ), 3.60 (apparent quin, $J=7.2 \mathrm{~Hz}$, $2 \mathrm{H}), 6.07$ (br s, 1H), 6.31 (br s, $1 \mathrm{H}), 7.41$ (dd, $J=0.9,7.3 \mathrm{~Hz}, 1 \mathrm{H}), 7.45$ (dd, $J=0.9,7.3 \mathrm{~Hz}$,

$1 \mathrm{H}), 7.50-7.62(\mathrm{~m}, 2 \mathrm{H} ; 2 \mathrm{H}$ of minor isomer overlapped), $7.75(\mathrm{dd}, J=1.0,7.1 \mathrm{~Hz}, 1 \mathrm{H}), 7.80$ $(\mathrm{dd}, J=1.0,7.2 \mathrm{~Hz}, 1 \mathrm{H}), 7.84(\mathrm{br} \mathrm{d}, J=7.8 \mathrm{~Hz}, 1 \mathrm{H}), 7.88$ (br d, $J=8.3 \mathrm{~Hz}, 1 \mathrm{H}$; minor isomer overlapped), 7.92 (br d, $J=$ $8.3 \mathrm{~Hz}, 1 \mathrm{H}), 8.30$ (br d, $J=8.2 \mathrm{~Hz}, 1 \mathrm{H}$; minor isomer overlapped). ${ }^{13} \mathbf{C} \mathbf{N M R}\left(\mathbf{1 0 0} \mathbf{~ M H z}, \mathbf{C D C l}_{3}\right): \delta 14.5,16.0,34.9,38.3$, 82.6, 85.3, 87.7, 89.0, 117.76, 118.81, 125.0, 125.1, 125.6, 125.8, 126.7, 126.8, 127.3, 127.5, 128.3, 128.5, 130.5, 130.8, 132.1, 132.4, 132.9, 133.0, 132.3 (minor isomer overlapped), 153.4, 156.2. IR (ATR-IR): 3247, 3057, 2975, 2934, 2873, 2207, 1625, 1586, 1541, 1396, 1300, 1262, 1229, 1148, 933, 913, 800, $772 \mathrm{~cm}^{-1}$. HRMS (ESI): $m / z$ calculated for $\mathrm{C}_{15} \mathrm{H}_{13} \mathrm{NNaO}^{+}[\mathrm{M}+\mathrm{Na}]^{+}=246.0889$, found: 246.0900 .

\section{N-ethyl-3-(naphthalen-2-yl)propiolamide (4b): General Protocol C}

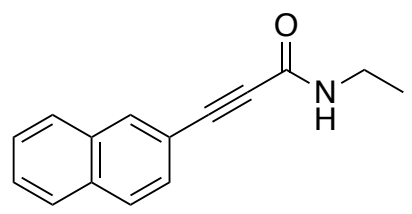

White solid, 17\% yield over 3 steps (378.9 mg) (10.2 mmol scale)

Ratio of streoisometric mixture: $1: 7.9 .{ }^{1} \mathbf{H}$ NMR $\left(400 \mathbf{~ M H z}, \mathbf{C D C l}_{3}\right): \delta 1.23(\mathrm{t}, J=7.3 \mathrm{~Hz}$, $3 \mathrm{H}), 1.31(\mathrm{t}, J=7.2 \mathrm{~Hz}, 3 \mathrm{H}), 3.43(\mathrm{dd}, J=1.3,7.3 \mathrm{~Hz}, 2 \mathrm{H}), 3.60$ (apparent quin, $J=7.1$ $\mathrm{Hz}, 2 \mathrm{H}), 5.72(\mathrm{br} \mathrm{s}, 1 \mathrm{H}), 5.95(\mathrm{br}, 1 \mathrm{H}), 7.51-7.58(\mathrm{~m}, 3 \mathrm{H} ; 3 \mathrm{H}$ of minor isomer overlapped), 7.80-7.84 (m, 3H; 3H of minor isomer overlapped), 8.08 (s, $1 \mathrm{H}), 8.12$ (s, $1 \mathrm{H}) .{ }^{13} \mathbf{C}$ NMR (100 MHz, $\left.\mathbf{C D C l}_{3}\right): \delta 14.6,16.0,34.9,38.3,80.9,83.3,84.8,91.1,117.4,117.5,126.85,126.93,127.6,127.7,127.80,127.83,127.98$, 128.03, 128.10 (minor isomer overlapped), 128.27, 128.34, 132.6 (minor isomer overlapped), 133.3, 133.4, 133.5, 133.6, 153.3, 156.1. IR (ATR-IR): 3267, 3056, 2975, 2934, 2211, 1624, 1536, 1439, 1355, 1296, 1269, 1251, 1212, 1178, 1149, 1128, 1057, 970, 935, 897, 862, 818, 746, $705 \mathrm{~cm}^{-1}$. HRMS (ESI): $m / z$ calculated for $\mathrm{C}_{15} \mathrm{H}_{13} \mathrm{NNaO}^{+}[\mathrm{M}+\mathrm{Na}]^{+}=$ 246.0889, found: 246.0901 .

\section{3-(benzo $[d][1,3]$ dioxol-5-yl)-N-ethylpropiolamide (4c): General Protocol C}

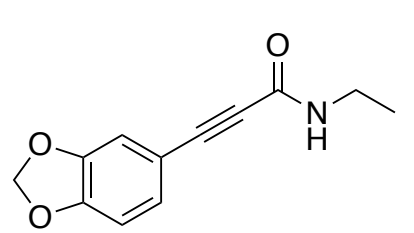

Yellowish solid, 52\% yield over 3 steps ( $848.1 \mathrm{mg})$ ( $7.48 \mathrm{mmol} \mathrm{scale})$

Ratio of streoisometric mixture: 1 : 7.9. ${ }^{1} \mathbf{H}$ NMR $\left(400 \mathbf{~ M H z} \mathbf{C D C l}_{3}\right): \delta 1.20(\mathrm{t}, J=7.3 \mathrm{~Hz}$, $3 \mathrm{H}), 1.26(\mathrm{t}, J=7.2 \mathrm{~Hz}, 3 \mathrm{H}), 3.38(\mathrm{dq}, J=1.4,7.3 \mathrm{~Hz}, 2 \mathrm{H}), 3.52$ (apparent quin, $J=7.2 \mathrm{~Hz}$, $2 \mathrm{H}), 5.77(\mathrm{br} \mathrm{s}, 1 \mathrm{H}), 5.95(\mathrm{br} \mathrm{s}, 1 \mathrm{H}), 6.00(\mathrm{~s}, 2 \mathrm{H}), 6.02(\mathrm{~s}, 1 \mathrm{H}), 6.78(\mathrm{~d}, J=8.0 \mathrm{~Hz}, 1 \mathrm{H})$, $6.80(\mathrm{~d}, J=1.5 \mathrm{~Hz}, 1 \mathrm{H}), 6.95(\mathrm{~d}, J=1.4 \mathrm{~Hz}, 1 \mathrm{H}), 6.98(\mathrm{~d}, J=1.4 \mathrm{~Hz}, 1 \mathrm{H}), 7.07$ (dd, $J=1.6$, $8.0 \mathrm{~Hz}, 1 \mathrm{H}), 7.12(\mathrm{dd}, J=1.5,8.0 \mathrm{~Hz}, 1 \mathrm{H}) .{ }^{13} \mathbf{C} \mathbf{N M R}\left(\mathbf{1 0 0} \mathbf{M H z}, \mathbf{C D C l}_{3}\right): \delta 14.6,16.0,34.9,38.3,79.6,81.9,84.7,91.1$, 101.6, 101.7, 108.6, 108.7, 112.1, 112.2, 113.25, 113.33, 127.9, 128.1, 147.5, 147.6, 149.4, 149.7, 153.5, 156.3. IR (ATR-IR): 3248, 3049, 2977, 2899, 2211, 1734, 1616, 1541, 1503, 1488, 1441, 1341, 1303, 1238, 1148, 1104, 1035, 961, 
932, 896, 860, 810, 733, 613, 571, 538, $510 \mathrm{~cm}^{-1}$. HRMS (ESI): $\mathrm{m} / z$ calculated for $\mathrm{C}_{12} \mathrm{H}_{11} \mathrm{NNaO}_{3}{ }^{+}[\mathrm{M}+\mathrm{Na}]^{+}=240.0631$, found: 240.0639 .

\section{N-ethyl-3-(4-(methylthio)phenyl)propiolamide (4e): General Protocol C}

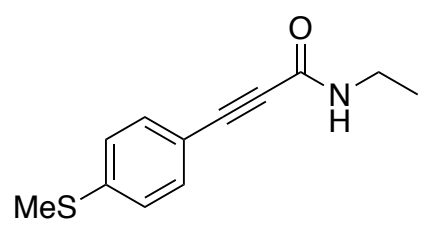

White solid, 33\% yield over 3 steps ( $485.2 \mathrm{mg})(6.79 \mathrm{mmol} \mathrm{scale})$

Ratio of streoisometric mixture: $1: 8.3 .{ }^{1} \mathbf{H}$ NMR (400 $\left.\mathbf{~ M H z}, \mathbf{C D C l}_{3}\right): \delta 1.21(\mathrm{t}, J=7.3$ $\mathrm{Hz}, 3 \mathrm{H}), 1.26(\mathrm{t}, J=7.2 \mathrm{~Hz}, 3 \mathrm{H}), 2.49(\mathrm{~s}, 3 \mathrm{H}), 2.50(\mathrm{~s}, 3 \mathrm{H}), 3.40(\mathrm{dd}, J=1.4,7.3 \mathrm{~Hz}, 2 \mathrm{H})$, 3.53 (apparent quin, $J=7.2 \mathrm{~Hz}, 2 \mathrm{H}), 5.83(\mathrm{br} \mathrm{s}, 1 \mathrm{H}), 6.00(\mathrm{br} \mathrm{s}, 1 \mathrm{H}), 7.18(\mathrm{~d}, J=8.5 \mathrm{~Hz}$, $2 \mathrm{H}), 7.20(\mathrm{~d}, J=8.5 \mathrm{~Hz}, 2 \mathrm{H}), 7.42(\mathrm{~d}, J=8.5 \mathrm{~Hz}, 2 \mathrm{H}), 7.46(\mathrm{~d}, J=8.5 \mathrm{~Hz}, 2 \mathrm{H}) .{ }^{13} \mathbf{C}$ NMR

(100 MHz, $\mathbf{C D C l}_{3}$ ): $\delta 14.6$ (minor isomer overlapped), 14.9, 15.9, 34.8, 38.2, 80.8, 83.2, 84.4, 90.7, 125.4 (minor isomer overlapped), 132.65, 132.74, 142.0, 142.4, 153.3, 156.1. IR (ATR-IR): 3265, 3058, 2974, 2933, 2925, 2873, 2249, 2210 , 1627, 1589, 1540, 1491, 1458, 1437, 1399, 1357, 1302, 1225, 1184, 1150, 1089, 1013, 948, 913, 817, $529 \mathrm{~cm}^{-1}$. HRMS (ESI): $m / z$ calculated for $\mathrm{C}_{12} \mathrm{H}_{13} \mathrm{NNaOS}^{+}[\mathrm{M}+\mathrm{Na}]^{+}=242.0610$, found: 242.0621 .

\section{3-(4-(diphenylamino)phenyl)- $\mathrm{N}$-ethylpropiolamide (4f): General Protocol D}

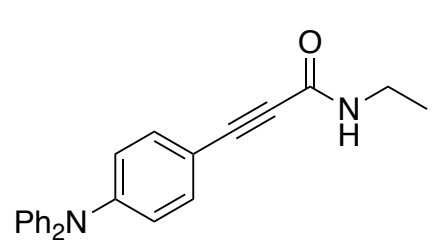

Orange solid, 64\% yield over 3 steps ( $803.7 \mathrm{mg})$ ( $4.0 \mathrm{mmol} \mathrm{scale})$

Ratio of streoisometric mixture: $1: 7.4 .{ }^{1} \mathbf{H}$ NMR (400 $\left.\mathbf{~ M H z}, \mathbf{C D C l}_{3}\right): \delta 1.20(\mathrm{t}, J=7.2$ $\mathrm{Hz}, 3 \mathrm{H}), 1.25(\mathrm{t}, J=7.2 \mathrm{~Hz}, 3 \mathrm{H}), 3.39(\mathrm{dq}, J=1.4,7.2 \mathrm{~Hz}, 2 \mathrm{H}), 3.52$ (apparent quin, $J=$ $7.2 \mathrm{~Hz}, 2 \mathrm{H}), 5.69(\mathrm{br} \mathrm{s}, 1 \mathrm{H}), 5.88(\mathrm{br} \mathrm{s}, 1 \mathrm{H}), 6.94(\mathrm{~d}, J=8.8 \mathrm{~Hz}, 2 \mathrm{H}), 6.96(\mathrm{~d}, J=8.8 \mathrm{~Hz}$, $2 \mathrm{H}), 7.08-7.12(\mathrm{~m}, 6 \mathrm{H} ; 6 \mathrm{H}$ of minor isomer overlapped), 7.27-7.31 $(\mathrm{m}, 4 \mathrm{H} ; 4 \mathrm{H}$ of minor isomer overlapped), 7.34 (d, $J=8.8 \mathrm{~Hz}, 2 \mathrm{H}), 7.38(\mathrm{~d}, J=8.8 \mathrm{~Hz}, 2 \mathrm{H}) \cdot{ }^{13} \mathbf{C}$ NMR (100 $\left.\mathbf{~ M H z}, \mathbf{C D C l}_{3}\right): \delta 14.6,34.8,82.6$, 85.4, 111.8, 120.9, 124.2, 125.6, 129.5, 133.6, 146.7, 149.5, 153.7. IR (ATR-IR): 3262, 3061, 3036, 2975, 2933, 2875, 2202, 1624, 1586, 1541, 1505, 1487, 1457, 1314, 1280, 1225, 1188, 1175, 1153, 1075, 1028, 941, 910, 829, 755, 730, 696, $647,619,567,538,510 \mathrm{~cm}^{-1}$. HRMS (ESI): $\mathrm{m} / z$ calculated for $\mathrm{C}_{23} \mathrm{H}_{20} \mathrm{~N}_{2} \mathrm{NaO}^{+}[\mathrm{M}+\mathrm{Na}]^{+}=363.1468$, found: 363.14687 .

\section{3-(4-(9H-carbazol-9-yl)phenyl)-N-ethylpropiolamide (4g): General Protocol D}

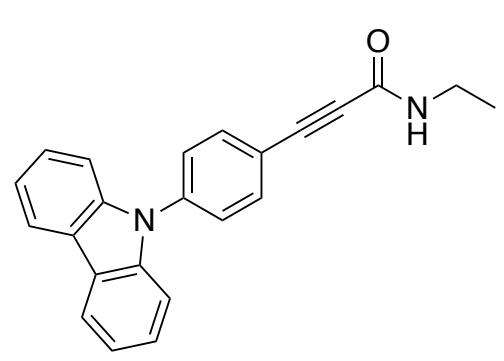

Brown solid, $49 \%$ over 3 steps $(824.6 \mathrm{mg})(5.0 \mathrm{mmol})$

Ratio of streoisometric mixture: $1: 8.2 .{ }^{1} \mathbf{H}$ NMR (400 $\left.\mathbf{M H z}, \mathbf{C D C l}_{3}\right): \delta 1.24(\mathrm{t}, J=7.3$ $\mathrm{Hz}, 3 \mathrm{H}), 1.31(\mathrm{t}, J=7.2 \mathrm{~Hz}, 3 \mathrm{H}), 3.43(\mathrm{dq}, J=1.3,7.3 \mathrm{~Hz}, 2 \mathrm{H}), 3.59$ (apparent quin, $J=7.1 \mathrm{~Hz}, 2 \mathrm{H}), 5.90(\mathrm{br} \mathrm{s}, 1 \mathrm{H}), 6.05(\mathrm{br} \mathrm{s}, 1 \mathrm{H}), 7.28-7.34(\mathrm{~m}, 2 \mathrm{H} ; 2 \mathrm{H}$ of minor isomer overlapped), 7.38-7.44 (m, 4H; 4H of minor isomer overlapped), 7.59 (d, J=8.5 Hz, $2 \mathrm{H}), 7.61(\mathrm{~d}, J=8.5 \mathrm{~Hz}, 2 \mathrm{H}), 7.75(\mathrm{~d}, J=8.5 \mathrm{~Hz}, 2 \mathrm{H}), 7.79(\mathrm{~d}, J=8.5 \mathrm{~Hz}, 2 \mathrm{H}), 8.13$ (br d, $J=7.7 \mathrm{~Hz}, 2 \mathrm{H} ; 2 \mathrm{H}$ of minor isomer overlapped). ${ }^{13} \mathbf{C}$ NMR (100 MHz, $\mathbf{C D C l}_{3}$ ): $\delta$ 14.5, 16.0, 34.9, 38.3, 81.4, 83.6, 83.8, 89.8, 109.6 (minor isomer overlapped), 118.81, 118.89, 120.37 (minor isomer overlapped), 120.4, 120.5, 123.65, 123.68, 126.1 (minor isomer overlapped), 126.7, 126.8, 134.0, 134.1, 139.3, 139.5, 140.2 (minor isomer overlapped), 153.1, 155.9. IR (ATR-IR): 3248, 3052, 2975, 2934, 2873, 2214, 1625, 1600, 1541, 1510, 1478, 1448, 1362, 1335, 1315, 1297, 1225, 1171, 1149, 1120, 1106, 1057, 1016, 937, 915, 836, 804, 748, 722, $700,624,565,539 \mathrm{~cm}^{-1}$. HRMS (ESI): $m / z$ calculated for $\mathrm{C}_{23} \mathrm{H}_{18} \mathrm{~N}_{2} \mathrm{NaO}^{+}[\mathrm{M}+\mathrm{Na}]^{+}=361.1311$, found: 361.1305 . 


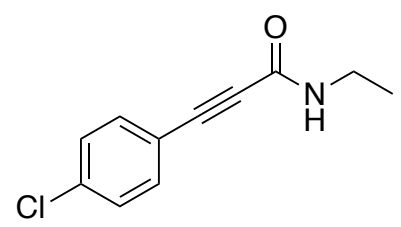

White solid, $21 \%$ yield over 3 steps ( $533.8 \mathrm{mg})$ ( $7.93 \mathrm{mmol} \mathrm{scale})$

Ratio of streoisometric mixture: $1: 9.0 .{ }^{1} \mathbf{H}$ NMR $\left(400 \mathbf{M H z} \mathbf{C D C l}_{3}\right): \delta 1.21(\mathrm{t}, J=7.3 \mathrm{~Hz}$, $3 \mathrm{H}), 1.26(\mathrm{t}, J=7.2 \mathrm{~Hz}, 3 \mathrm{H}), 3.40(\mathrm{dq}, J=1.4,7.3 \mathrm{~Hz}, 2 \mathrm{H}), 3.52$ (apparent quin, $J=7.2 \mathrm{~Hz}$, $2 \mathrm{H}), 5.95(\mathrm{br} \mathrm{s}, 1 \mathrm{H}), 6.09$ (br s, $1 \mathrm{H}), 7.33(\mathrm{~d}, J=8.6 \mathrm{~Hz}, 1 \mathrm{H}), 7.36(\mathrm{~d}, J=8.6 \mathrm{~Hz}, 1 \mathrm{H}), 7.44$ $(\mathrm{d}, J=8.6 \mathrm{~Hz}, 1 \mathrm{H}), 7.49$ (d, $J=8.6 \mathrm{~Hz}, 1 \mathrm{H}) .{ }^{13} \mathbf{C}$ NMR (100 MHz, $\left.\mathbf{C D C l}_{3}\right): \delta$ 14.5, 15.9, 34.9, 38.3, 81.4, 83.1, 83.9, 89.3, 118.66, 118.71, 128.9, 129.0, 133.6, 133.7, 136.3, 136.6, 153.0, 155.8. IR (ATR-IR): 3276, 3058, 2979, 2932, 2872, 2249, 2218, 1623, 1590, 1541, 1488, 1397, 1355, 1299, 1218, 1150, 1088, 1011, 948, 913 , $829,702,529 \mathrm{~cm}^{-1}$. HRMS (ESI): $m / z$ calculated for $\mathrm{C}_{11} \mathrm{H}_{10} \mathrm{ClNNaO}^{+}[\mathrm{M}+\mathrm{Na}]^{+}=230.0343$, found: 230.0350 .

\section{N-ethyl-3-(4-(trifluoromethyl)phenyl)propiolamide (4i): General Protocol D}

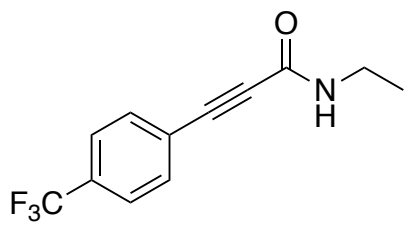

White solid, $23 \%$ yield $(545.1 \mathrm{mg})(7.64 \mathrm{mmol}$ scale $)$

Ratio of streoisometric mixture: $1: 9.6 .{ }^{1} \mathbf{H}$ NMR $\left(\mathbf{4 0 0} \mathbf{~ M H z}, \mathbf{C D C l}_{3}\right): \delta 1.23(\mathrm{t}, J=7.2 \mathrm{~Hz}$, $3 \mathrm{H}), 1.28(\mathrm{t}, J=7.2 \mathrm{~Hz}, 3 \mathrm{H}), 3.42(\mathrm{dq}, J=1.4,7.2 \mathrm{~Hz}, 2 \mathrm{H}), 3.54$ (apparent quin, $J=7.2$ $\mathrm{Hz}, 2 \mathrm{H}), 5.85$ (br s, $1 \mathrm{H}), 6.02(\mathrm{br} \mathrm{s}, 1 \mathrm{H}), 7.62-7.66(\mathrm{~m}, 4 \mathrm{H} ; 4 \mathrm{H}$ of minor isomer overlapped). ${ }^{13} \mathbf{C}$ NMR (100 $\mathbf{~ M H z}, \mathbf{C D C l}_{3}$ ): $\delta 14.5,34.9,82.4,84.8,123.5$ (q, $J=270.9$

$\mathrm{Hz}), 124.1,125.4(\mathrm{q}, J=3.8 \mathrm{~Hz}), 131.6(\mathrm{q}, J=32.7 \mathrm{~Hz}), 132.6,152.7 .{ }^{19} \mathbf{F}$ NMR $\left(376 \mathbf{~ M H z}, \mathbf{C D C l}_{3}\right): \delta-63.12,-63.11$. IR (ATR-IR): 3290, 2991, 2949, 2888, 2222, 1623, 1541, 1457, 1402, 1320, 1292, 1225, 1164, 1119, 1106, 1067, 1012, 957, 843, 740, 670, 598, $564 \mathrm{~cm}^{-1}$. HRMS (ESI): $\mathrm{m} / z$ calculated for $\mathrm{C}_{12} \mathrm{H}_{10} \mathrm{~F}_{3} \mathrm{NNaO}^{+}[\mathrm{M}+\mathrm{Na}]^{+}=264.0607$, found: 264.0609 .

ethyl 2-(3-(ethylamino)-3-oxoprop-1-yn-1-yl)benzoate (4j)

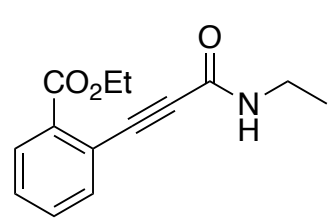

In analogy to the protocol by T. Eckert and J. Ipaktshi, ${ }^{[11]}$ ethyl 2-iodobenzoate $(1.0 \mathrm{mmol}$, $0.25 \mathrm{~g}$, 1.0 eq.), $\mathrm{N}$-ethylpropiolamide ( $4 \mathrm{mmol}, 0.39 \mathrm{~g}$, 4 eq.) was dissolved in THF $(3.0 \mathrm{~mL})$ in an oven-dried $20 \mathrm{~mL}$ Schlenk tube. Then, $\mathrm{PdCl}_{2}\left(\mathrm{PPh}_{3}\right)_{2}(0.02 \mathrm{mmol}, 14 \mathrm{mg}, 2 \mathrm{~mol} \%), \mathrm{CuI}$ (0.04 mmol, $7.6 \mathrm{mg}, 4 \mathrm{~mol} \%$ ), and $\mathrm{K}_{2} \mathrm{CO}_{3}\left(2.0 \mathrm{mmol}, 0.28 \mathrm{~g}\right.$ ) were added and heated to $65^{\circ} \mathrm{C}$ for $22 \mathrm{~h}$. After being cooled to rt, the mixture was diluted with EtOAc and filtered through a short pad of silica gel. Purification by silica gel column chromatography (pre-adsorped, hex/EtOAc 3:1 to 2:1) gave an off-white solid, $70 \%$ yield (193.9 mg).

Ratio of streoisometric mixture: $1: 8.9$ (minor isomer was expressed by italic)

${ }^{1}$ H NMR (400 MHz, $\left.\mathbf{C D C l}_{3}\right): \delta 1.21(\mathrm{t}, J=7.2 \mathrm{~Hz}, 3 \mathrm{H}), 1.26(\mathrm{t}, J=7.2 \mathrm{~Hz}, 3 \mathrm{H}), 1.41(\mathrm{t}, J=7.2 \mathrm{~Hz}, 3 \mathrm{H}), 1.43(\mathrm{t}, J=7.2$ $\mathrm{Hz}, 3 \mathrm{H}), 3.41(\mathrm{dd}, J=1.3,7.2 \mathrm{~Hz}, 2 \mathrm{H}), 3.41(\mathrm{dq}, J=1.3,7.2 \mathrm{~Hz}, 2 \mathrm{H}), 3.63$ (apparent quin, $J=7.2 \mathrm{~Hz}, 2 \mathrm{H}), 4.40(\mathrm{q}, J=$ $7.2 \mathrm{~Hz}, 2 \mathrm{H}), 4.40(\mathrm{q}, J=7.2 \mathrm{~Hz}, 2 \mathrm{H}), 5.80(\mathrm{br} \mathrm{s}, 1 \mathrm{H}), 6.19(\mathrm{br} \mathrm{s}, 1 \mathrm{H}), 7.45-7.49(\mathrm{~m}, 1 \mathrm{H}), 7.50-7.54(\mathrm{~m}, 1 \mathrm{H} ; 2 \mathrm{H}$ of minor isomer overlapped), 7.64-7.66 $(\mathrm{m}, 1 \mathrm{H}), 7.69-7.71(\mathrm{~m}, 1 \mathrm{H}), 8.00-8.02\left(\mathrm{~m}, 1 \mathrm{H} ; 1 \mathrm{H}\right.$ of minor isomer overlapped). ${ }^{13} \mathbf{C}$ NMR (100 MHz, $\left.\mathbf{C D C l}_{3}\right): \delta$ 14.2, 14.6, 34.8, 61.5, 82.9, 87.5, 121.0, 129.5, 130.6, 131.9, 132.8, 134.8, 153.3, 165.4. IR (ATR-IR): 3283, 3069, 2979, 2935, 2902, 2875, 2217, 1715, 1628, 1524, 1486, 1445, 1366, 1286, 1254, 1218, 1136, 1080, 1041, 1016, 942, 856, 801, 757, 698, $666 \mathrm{~cm}^{-1}$. HRMS (ESI): $m / z$ calculated for $\mathrm{C}_{14} \mathrm{H}_{15} \mathrm{NNaO}_{3}{ }^{+}[\mathrm{M}+\mathrm{Na}]^{+}=$ 268.0944, found: 268.0949 . 


\section{3-(4-cyanophenyl)-N-ethylpropiolamide (4k): General Protocol B}

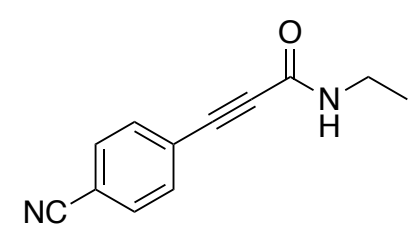

Slightly yellow solid, $28 \%$ yield $(42.1 \mathrm{mg})(0.76 \mathrm{mmol} \mathrm{scale})$

Ratio of streoisometric mixture: 1 : 9.6. ${ }^{1} \mathbf{H}$ NMR $\left(400 \mathbf{~ M H z} \mathbf{C D C l}_{3}\right): \delta 1.23(\mathrm{t}, J=7.3 \mathrm{~Hz}$, $3 \mathrm{H}), 1.28(\mathrm{t}, J=7.2 \mathrm{~Hz}, 3 \mathrm{H}), 3.42(\mathrm{dd}, J=1.4,7.3 \mathrm{~Hz}, 2 \mathrm{H}), 3.54$ (apparent quin, $J=7.2$ $\mathrm{Hz}, 2 \mathrm{H}), 5.87$ (br s, $1 \mathrm{H}), 5.96(\mathrm{br} \mathrm{s}, 1 \mathrm{H}), 7.61(\mathrm{~d}, J=8.5 \mathrm{~Hz}, 2 \mathrm{H}), 7.66(\mathrm{~d}, J=8.5 \mathrm{~Hz}, 2 \mathrm{H}$; $2 \mathrm{H}$ of minor isomer overlapped). ${ }^{13} \mathbf{C}$ NMR (100 $\left.\mathbf{~ M H z}, \mathbf{C D C l}_{3}\right): \delta 14.6,16.0,35.0,38.3$, 81.9, 83.9, 86.3, 87.9, 113.5, 113.7, 118.0 (minor isomer overlapped), 125.08, 125.15, 132.20, 132.24, 132.88, 132.91, 152.4, 155.1. IR (ATR-IR): 3218, 3046, 2982, 2938, 2877, 2858, 2228, 1622, 1558, 1507, 1457, 1437, 1305, 1231, 1152, 938, 913, 848, 831, 730, $557 \mathrm{~cm}^{-1}$. HRMS (ESI): $m / z$ calculated for $\mathrm{C}_{12} \mathrm{H}_{10} \mathrm{~N}_{2} \mathrm{NaO}^{+}[\mathrm{M}+\mathrm{Na}]^{+}=221.0685$, found: 221.0682 .

\section{N-ethyl-3-(4-nitrophenyl)propiolamide (41)}

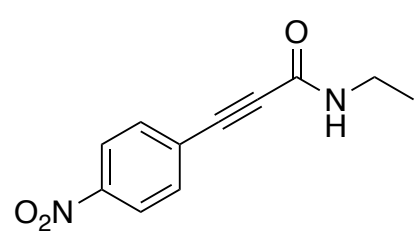

In analogy to the protocol by T. Eckert and J. Ipaktshi, ${ }^{11} 1$-iodo-4-nitrobenzene $(1.0 \mathrm{mmol}$, $0.25 \mathrm{~g}, 1.0$ eq.), $N$-ethylpropiolamide $(4.0 \mathrm{mmol}, 0.39 \mathrm{~g}, 4$ eq.) was dissolved in THF ( 3.0 $\mathrm{mL})$ in an oven-dried $20 \mathrm{~mL}$ Schlenk tube. Then, $\mathrm{PdCl}_{2}\left(\mathrm{PPh}_{3}\right)(0.02 \mathrm{mmol}, 14 \mathrm{mg}, 2$ mol\%), $\mathrm{CuI}(0.04 \mathrm{mmol}, 7.6 \mathrm{mg}, 4 \mathrm{~mol} \%), \mathrm{K}_{2} \mathrm{CO}_{3}(2.0 \mathrm{mmol}, 0.28 \mathrm{~g})$ were added and heated to $65^{\circ} \mathrm{C}$. After stirring for $12 \mathrm{~h}$, the reaction was cooled, diluted with EtOAc, and filtered through a short pad of silica gel. Purification with preparative recycling HPLC followed by silica gel column chromatography (pre-adsorped, hex/EtOAc 2:1 to 3:2) gave a white solid, 47\% yield (102.5 mg).

Ratio of streoisometric mixture: $1: 10.1{ }^{1}{ }^{\mathbf{H}} \mathbf{N M R}\left(\mathbf{4 0 0} \mathbf{~ M H z}, \mathbf{C D C l}_{3}\right): \delta 1.23(\mathrm{t}, J=7.3 \mathrm{~Hz}, 3 \mathrm{H}), 1.29(\mathrm{t}, J=7.2 \mathrm{~Hz}, 3 \mathrm{H})$, $3.43(\mathrm{dq}, J=1.4,7.3 \mathrm{~Hz}, 2 \mathrm{H}), 3.55$ (apparent quin, $J=7.2 \mathrm{~Hz}, 2 \mathrm{H}), 5.95($ br s, $1 \mathrm{H}), 6.08($ br s, $1 \mathrm{H}), 7.68(\mathrm{~d}, J=8.9 \mathrm{~Hz}$, $2 \mathrm{H}), 7.72(\mathrm{~d}, J=8.9 \mathrm{~Hz}, 2 \mathrm{H}), 8.23(\mathrm{~d}, J=8.9 \mathrm{~Hz}, 2 \mathrm{H}), 8.26(\mathrm{~d}, J=8.9 \mathrm{~Hz}, 2 \mathrm{H}) .{ }^{13} \mathbf{C}$ NMR $\left(\mathbf{1 0 0 ~} \mathbf{M H z}, \mathbf{C D C l}_{3}\right): \delta 14.5$, 16.0, 35.0, 38.3, 81.5, 84.3, 86.9, 87.5, 123.68, 123.73, 126.88, 126.98, 133.20, 133.24, 148.1, 148.2, 152.3, 155.0. IR (ATR-IR): 3280, 3106, 3073, 2978, 2940, 2880, 2852, 2219, 1629, 1593, 1542, 1517, 1457, 1401, 1375, 1344, 1287, 1223, 1152, 1108, 1011, 953, 913, 857, 749, 686, $520 \mathrm{~cm}^{-1}$. HRMS (ESI): $m / z$ calculated for $\mathrm{C}_{11} \mathrm{H}_{10} \mathrm{~N}_{2} \mathrm{NaO}_{3}{ }^{+}[\mathrm{M}+\mathrm{Na}]^{+}=$ 241.0584, found: 241.0587.

\section{N-ethyl-3-mesitylpropiolamide (4m): General Protocol D}

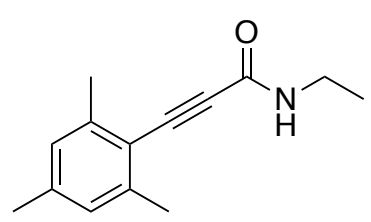

White solid, $65 \%$ yield over 3 steps $(927.4 \mathrm{mg})(7.0 \mathrm{mmol} \mathrm{scale})$

Ratio of streoisometric mixture: $1: 5.3 .{ }^{1} \mathbf{H}$ NMR $\left(400 \mathbf{~ M H z} \mathbf{C D C l}_{3}\right): \delta 1.21(\mathrm{t}, J=7.2 \mathrm{~Hz}$, $3 \mathrm{H}), 1.26(\mathrm{t}, J=7.2 \mathrm{~Hz}, 3 \mathrm{H}), 2.28(\mathrm{~s}, 3 \mathrm{H}), 2.30(\mathrm{~s}, 3 \mathrm{H}), 2.41(\mathrm{~s}, 6 \mathrm{H}), 2.44(\mathrm{~s}, 6 \mathrm{H}), 3.40(\mathrm{dq}$, $J=1.4,7.2 \mathrm{~Hz}, 2 \mathrm{H}$ ), 3.55 (apparent quin, $J=7.2 \mathrm{~Hz}, 2 \mathrm{H}), 5.94(\mathrm{br} \mathrm{s}, 1 \mathrm{H}), 6.00$ (br s, $1 \mathrm{H}$ ), 6.86 (s, 2H), 6.90 (s, 2H). ${ }^{13} \mathbf{C}$ NMR (100 $\left.\mathbf{~ M H z}, \mathbf{C D C l}_{3}\right): \delta 14.5,15.9,20.7,20.8,21.29$, 21.33, 34.7, 38.1, 82.5, 88.3, 88.9, 90.6, 117.0 (minor isomer overlapped), 127.7, 127.8, 139.5, 140.0, 141.4, 141.7, 153.7, 156.6. IR (ATR-IR): 3247, 3044, 2975, 2933, 2921, 2875, 2203, 1625, 1540, 1473, 1457, 1377, 1355, 1308, 1284, 1223, 1148, 1034, 962, 930, 852, 790, 726, $669 \mathrm{~cm}^{-1}$. HRMS (ESI): $m / z$ calculated for $\mathrm{C}_{14} \mathrm{H}_{17} \mathrm{NNaO}^{+}[\mathrm{M}+\mathrm{Na}]^{+}=238.1202$, found: 238.1204. 


\section{N-ethyl-3-(thiophen-3-yl)propiolamide (4n): General Protocol D}

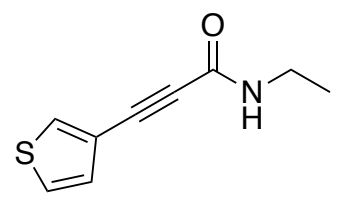

Slightly yellowish solid, $24 \%$ over 3 steps ( $428.8 \mathrm{mg}$ ) ( $10 \mathrm{mmol} \mathrm{scale})$

Ratio of streoisometric mixture: 1 : 8.6. ${ }^{1} \mathbf{H}$ NMR $\left(400 \mathbf{~ M H z}, \mathbf{C D C l}_{3}\right): \delta 1.20(\mathrm{t}, J=7.3 \mathrm{~Hz}, 3 \mathrm{H})$, $1.25(\mathrm{t}, J=7.2 \mathrm{~Hz}, 3 \mathrm{H}), 3.39(\mathrm{dq}, J=1.4,7.3 \mathrm{~Hz}, 2 \mathrm{H}), 3.52$ (apparent quin, $J=7.2 \mathrm{~Hz}, 2 \mathrm{H}$ ), $5.94($ br s, $1 \mathrm{H}), 6.07$ (br s, $1 \mathrm{H}), 7.17(\mathrm{dd}, J=1.1,5.0 \mathrm{~Hz}, 1 \mathrm{H}), 7.21(\mathrm{dd}, J=1.1,5.0 \mathrm{~Hz}, 1 \mathrm{H})$, $7.30(\mathrm{dd}, J=3.0,5.0 \mathrm{~Hz}, 1 \mathrm{H}), 7.33(\mathrm{dd}, J=3.0,5.0 \mathrm{~Hz}, 1 \mathrm{H}), 7.64(\mathrm{dd}, J=1.1,3.0 \mathrm{~Hz}, 1 \mathrm{H}), 7.69(\mathrm{dd}, J=1.1,3.0 \mathrm{~Hz}, 1 \mathrm{H})$. ${ }^{13}$ C NMR (100 MHz, $\left.\mathbf{C D C l}_{3}\right): \delta$ 14.5, 15.8, 34.8, 38.2, 79.8, 80.6, 83.0, 86.1, 119.3, 119.4, 125.8, 126.0, 128.4, 129.8, 132.1, 132.5, 153.3, 156.1. IR (ATR-IR): 3253, 3107, 3083, 3050, 2976, 2934, 2875, 2217, 1621, 1540, 1509, 1457, 1448, 1377, 1360, 1282, 1222, 1173, 1148, 1080, 1058, 971, 898, 868, 824, 783, 692, 670, 625, $602 \mathrm{~cm}^{-1}$. HRMS (ESI): $m / z$ calculated for $\mathrm{C}_{9} \mathrm{H}_{9} \mathrm{NNaOS}^{+}[\mathrm{M}+\mathrm{Na}]^{+}=202.0297$, found: 202.0298 .

3-(benzo $[b]$ thiophen-2-yl)- $N$-ethylpropiolamide (4o): General Protocol C

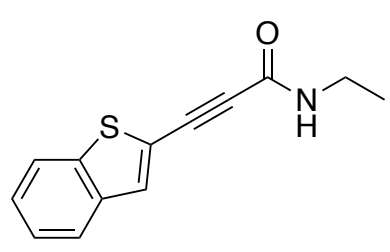

Off-white solid, $9 \%$ yield over 3 steps ( $143.1 \mathrm{mg})(7.2 \mathrm{mmol} \mathrm{scale})$

Ratio of streoisometric mixture: 1 : 9.7. ${ }^{1} \mathbf{H}$ NMR (400 $\left.\mathbf{M H z}, \mathbf{C D C l}_{3}\right): \delta 1.22(\mathrm{t}, J=7.3 \mathrm{~Hz}$, $3 \mathrm{H}), 1.28(\mathrm{t}, J=7.2 \mathrm{~Hz}, 3 \mathrm{H}), 3.42(\mathrm{dq}, J=1.4,7.3 \mathrm{~Hz}, 2 \mathrm{H}), 3.54$ (apparent quin, $J=7.2 \mathrm{~Hz}$, $2 \mathrm{H}), 5.90$ (br s, $1 \mathrm{H}), 6.10$ (br s, $1 \mathrm{H}), 7.36-7.43$ (m, $2 \mathrm{H} ; 2 \mathrm{H}$ of minor isomer overlapped), $7.61(\mathrm{~s}, 1 \mathrm{H}), 7.66(\mathrm{~s}, 1 \mathrm{H}), 7.76-7.81\left(\mathrm{~m}, 2 \mathrm{H} ; 2 \mathrm{H}\right.$ of minor isomer overlapped). ${ }^{13} \mathbf{C}$ NMR (100 MHz, $\mathbf{C D C l}_{3}$ ): $\delta$ 14.5, 15.9, 34.9, 38.2, 78.2, 84.3, 85.8, 88.1, 119.7, 119.9, 122.07, 122.11, 124.3, 124.4, 125.0, 125.1, 126.4, 126.5, 132.2, 132.5, 138.5 (minor isomer overlapped), 140.9, 141.1, 152.8, 155.5. IR (ATR-IR): 3262, 3057, 2975, 2933, 2874, 2207, 1626, 1541, 1508, 1456, 1435, 1355, 1333, 1278, 1191, 1153, 1069, 1014, 916, 860, 836, 746, 724, 696, $560 \mathrm{~cm}^{-1}$. HRMS (ESI): $\mathrm{m} / z$ calculated for $\mathrm{C}_{13} \mathrm{H}_{11} \mathrm{NNaOS}^{+}[\mathrm{M}+\mathrm{Na}]^{+}=252.0454$, found: 252.0460 .

\section{3-(dibenzo $[b, d]$ thiophen-2-yl)-N-ethylpropiolamide (4p): General Protocol C}

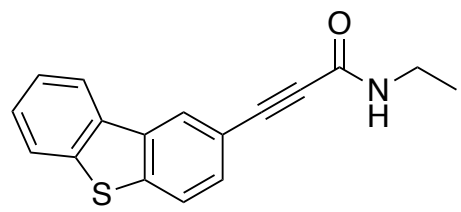

Off-white solid, $39 \%$ yield over 3 steps ( $353.1 \mathrm{mg})$ ( $3.23 \mathrm{mmol} \mathrm{scale})$

Ratio of streoisometric mixture: $1: 8.3 .{ }^{1} \mathbf{H}$ NMR (400 $\left.\mathbf{M H z}, \mathbf{C D C l}_{3}\right): \delta 1.24(\mathrm{t}, J=7.2$ $\mathrm{Hz}, 3 \mathrm{H}$ ), $1.30(\mathrm{t}, J=7.1 \mathrm{~Hz}, 3 \mathrm{H}$ ), 3.44 (apparent quin, $J=6.7 \mathrm{~Hz}, 2 \mathrm{H}$ ), 3.41 (apparent quin, $J=6.8 \mathrm{~Hz}, 2 \mathrm{H}), 5.95$ (br s, $1 \mathrm{H}), 6.18(\mathrm{br} \mathrm{s}, 1 \mathrm{H}), 7.43-7.49(\mathrm{~m}, 2 \mathrm{H} ; 2 \mathrm{H}$ of minor isomer overlapped), 7.53-7.59 (m, $1 \mathrm{H}$; minor isomer overlapped), 7.76-7.83 (m, $2 \mathrm{H} ; 2 \mathrm{H}$ of minor isomer overlapped), 8.06-8.12 (m, $1 \mathrm{H}$; minor isomer overlapped), $8.28(\mathrm{~s}, 1 \mathrm{H}), 8.31(\mathrm{~s}, 1 \mathrm{H}) .{ }^{13} \mathbf{C}$ NMR (100 MHz, $\left.\mathbf{C D C l}_{3}\right): \delta$ 14.6, 16.0, 34.9, 38.3, 80.7, 83.1, 84.7, 91.1, 116.0, 116.1, 121.6, 121.7, 122.77, 122.81, 122.86, 122.94, 124.79, 124.84, 127.36, 127.44, 129.9, 130.0, 134.5 (minor isomer overlapped), 135.56, 135.63, 139.6 (minor isomer overlapped), 141.3, 141.6, 153.4, 156.1. IR (ATR-IR): 3248, 3059, 2975, 2934, 2875, 2212, 1624, 1541, 1466, 1432, 1295, 1278, 1264, 1229, 1200, 1149, 1079, 1024, 949, 911, 886, 851, 813, 762, 730, 628, $611 \mathrm{~cm}^{-1}$. HRMS (ESI): $\mathrm{m} / z$ calculated for $\mathrm{C}_{17} \mathrm{H}_{13} \mathrm{NNaOS}^{+}[\mathrm{M}+\mathrm{Na}]^{+}=302.0610$, found: 302.0609 .

\section{3-(cyclohex-1-en-1-yl)-N-ethylpropiolamide (4r): General Protocol D}

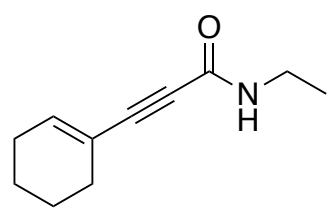

Off-white solid, $67 \%$ yield over 3 steps ( $1.0 \mathrm{~g})$ ( $10 \mathrm{mmol}$ scale)

Ratio of streoisometric mixture: $1: 6.8 .{ }^{1} \mathbf{H}$ NMR (400 $\left.\mathbf{~ M H z}, \mathbf{C D C l}_{3}\right): \delta 1.17(\mathrm{t}, J=7.3 \mathrm{~Hz}, 3 \mathrm{H})$, $1.21(\mathrm{t}, J=7.2 \mathrm{~Hz}, 3 \mathrm{H}), 1.57-1.67(\mathrm{~m}, 4 \mathrm{H} ; 4 \mathrm{H}$ of minor isomer overlapped), $2.10-2.18(\mathrm{~m}, 4 \mathrm{H}$; $4 \mathrm{H}$ of minor isomer overlapped), 3.35 (dd, $J=1.4,7.3 \mathrm{~Hz}, 2 \mathrm{H}$ ), 3.44 (apparent quin, $J=7.2 \mathrm{~Hz}$, 
2H), $5.78(\mathrm{br} \mathrm{s}, 1 \mathrm{H}), 5.86(\mathrm{br} \mathrm{s}, 1 \mathrm{H}), 6.32-6.34(\mathrm{~m}, 1 \mathrm{H}), 6.38-6.40(\mathrm{~m}, 1 \mathrm{H}) .{ }^{13} \mathbf{C}$ NMR $\left(\mathbf{1 0 0 ~} \mathbf{~ M H z}, \mathbf{C D C l}_{3}\right): \delta$ 14.6, 15.9, 21.2 (minor isomer overlapped), 22.0 (minor isomer overlapped), 25.87, 25.92, 28.3 (minor isomer overlapped), 34.8, 38.2, 78.5, 81.0, 86.5, 93.0, 118.8, 118.9, 140.0, 140.6, 153.8, 156.5. IR (ATR-IR): 3247, 3051, 2976, 2931, 2860, 2841, 2828, 2203, 1619, 1532, 1436, 1377, 1360, 1287, 1264, 1184, 1148, 1058, 918, 843, 799, 698, 651, $568 \mathrm{~cm}^{-1}$. HRMS (ESI): $m / z$ calculated for $\mathrm{C}_{11} \mathrm{H}_{15} \mathrm{NNaO}^{+}[\mathrm{M}+\mathrm{Na}]^{+}=200.1046$, found: 200.1048 .

(E)-N-ethyl-5-(4-(trifluoromethyl)phenyl)pent-4-en-2-ynamide (4s): General Protocol C

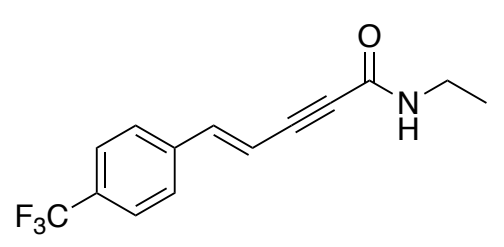

Off-white solid, $6 \%$ yield over 3 steps $(75.5 \mathrm{mg})$ ( $4.89 \mathrm{mmol} \mathrm{scale})$

Ratio of streoisometric mixture: $1: 8.0 .{ }^{1} \mathbf{H}$ NMR (400 $\left.\mathbf{~ M H z}, \mathbf{C D C l}_{3}\right): \delta 1.20(\mathrm{t}, J=$ $7.2 \mathrm{~Hz}, 3 \mathrm{H}), 1.26(\mathrm{t}, J=7.2 \mathrm{~Hz}, 3 \mathrm{H}), 3.39(\mathrm{dd}, J=1.3,7.2 \mathrm{~Hz}, 2 \mathrm{H}), 3.50$ (apparent quin, $J=7.2 \mathrm{~Hz}, 2 \mathrm{H}), 5.81(\mathrm{br} \mathrm{s}, 1 \mathrm{H}), 5.92(\mathrm{br} \mathrm{s}, 1 \mathrm{H}), 6.26(\mathrm{~d}, J=16.4 \mathrm{~Hz}, 1 \mathrm{H}), 6.34$ $(\mathrm{d}, J=16.3 \mathrm{~Hz}, 1 \mathrm{H}), 7.15(\mathrm{~d}, J=16.4 \mathrm{~Hz}, 1 \mathrm{H}), 7.20(\mathrm{~d}, J=16.3 \mathrm{~Hz}, 1 \mathrm{H}), 7.50(\mathrm{~d}, J=$ $8.2 \mathrm{~Hz}, 2 \mathrm{H} ; 2 \mathrm{H}$ of minor isomer overlapped), $7.60\left(\mathrm{~d}, J=8.1 \mathrm{~Hz}, 2 \mathrm{H} ; 2 \mathrm{H}\right.$ of minor isomer overlapped). ${ }^{13} \mathbf{C}$ NMR (100 MHz, $\left.\mathbf{C D C l}_{3}\right): \delta 14.5,34.8,83.0,85.9,108.1,123.8(\mathrm{q}, J=270.5 \mathrm{~Hz}) 125.8(\mathrm{q}, J=3.8 \mathrm{~Hz}), 126.8,131.1(\mathrm{q}, J=32.6 \mathrm{~Hz})$, 138.5, 143.9, 153.0. ${ }^{19} \mathbf{F}$ NMR (376 $\mathbf{~ M H z}, \mathbf{C D C l}_{3}$ ): $\delta$-62.8 (minor isomer overlapped). IR (ATR-IR): 3283, 3060, 2984, $2937,2875,2203,1625,1541,1326,1288,1268,1163,1110,1067,1014,960,949,888,863,819,684,667,600,519$ $\mathrm{cm}^{-1}$. HRMS (ESI): $\mathrm{m} / z$ calculated for $\mathrm{C}_{14} \mathrm{H}_{12} \mathrm{~F}_{3} \mathrm{NNaO}^{+}[\mathrm{M}+\mathrm{Na}]^{+}=290.0763$, found: 290.0768 .

\section{$N$-ethylbut-2-ynamide (4t): General Protocol B}

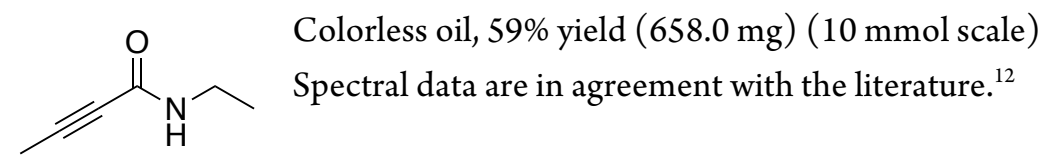

\section{$N$-ethylpropiolamide $(4 \mathrm{u})$}<smiles>C#CC(=O)NCC</smiles>

To a solution of ethylamine in $\mathrm{MeOH}(44.9 \mathrm{mmol}, 2.02 \mathrm{~g}, 6.7 \mathrm{~mL}, 1.1 \mathrm{eq}$.) was added methyl propiolate ( $40.8 \mathrm{mmol}, 3.43 \mathrm{~g}, 1.0 \mathrm{eq}$.) at $-78^{\circ} \mathrm{C}$ and stirred at the same temperature for $3.5 \mathrm{~h}$. Then, the reaction warmed to rt and volatiles were azeotropically removed with toluene in vacuo. Bulb-to-bulb distillation $\left(4.0\right.$ Torr, $\left.175^{\circ} \mathrm{C}\right)$ gave a colorless oil in $58 \%$ yield $(2.31 \mathrm{~g})$. Spectral data are in agreement with the literature. $^{13}$

\section{N-ethyl-3-(triisopropylsilyl)propiolamide (4v): General Protocol D}

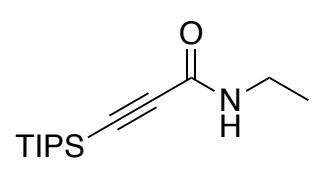

White solid, 19\% yield over 3 steps (493.2 $\mathrm{mg}$ ) (10 mmol scale)

Ratio of streoisometric mixture: 1 : 5.5. ${ }^{1} \mathbf{H}$ NMR (400 $\left.\mathbf{~ M H z}, \mathbf{C D C l}_{3}\right): \delta 1.09-1.12(\mathrm{~m}, 21 \mathrm{H}$; minor isomer overlapped), $1.18(\mathrm{t}, J=7.3 \mathrm{~Hz}, 3 \mathrm{H}), 1.22(\mathrm{t}, J=7.2 \mathrm{~Hz}, 3 \mathrm{H}), 3.34(\mathrm{dq}, J=1.4,7.3$ $\mathrm{Hz}, 2 \mathrm{H}), 3.48$ (apparent quin, $J=7.2 \mathrm{~Hz}, 2 \mathrm{H}), 5.97$ (br s, $1 \mathrm{H}), 6.09$ (br s, $1 \mathrm{H}) .{ }^{13} \mathbf{C}$ NMR (100 $\mathbf{M H z}, \mathbf{C D C l}_{3}$ ): $\delta 10.9$ (minor isomer overlapped), 14.5, 16.0, 18.4 (minor isomer overlapped), 34.7, 38.2, 87.7, 94.7, 97.2, 99.9, 152.6, 155.5. IR (ATR-IR): 3264, 3052, 2942, 2892, 2867, 1633, 1541, 1458, 1276, 1149, 1065, 997, 943, 914, 883, $789,744,709,677,582 \mathrm{~cm}^{-1}$. HRMS (ESI): $\mathrm{m} / z$ calculated for $\mathrm{C}_{14} \mathrm{H}_{27} \mathrm{NNaOSi}^{+}[\mathrm{M}+\mathrm{Na}]^{+}=276.1754$, found: 276.1747 . 


\section{2-2. Optimization of Reaction Conditions (Table S1)}

Reaction between alkynamide $\mathbf{1 b}$ and alkynylboronate $\mathbf{2 a}$ affected by ${ }^{\mathrm{n}} \mathrm{BuLi}$ gave the product $\mathbf{3 b}$ in $63 \%$ yield (entry 1 ). Hydride bases are also effective and $\mathbf{3 b}$ were obtained in comparable yields (entry 2, 3). Anionic activation of amide $\mathbf{1 b}$ was mandatory (entry 4). Yield was improved by using a slight excess of ${ }^{n} \mathrm{BuLi}$ (entry 5) and running the reaction at $110{ }^{\circ} \mathrm{C}$ (entry 6). Lowering the reaction temperature to $\mathrm{rt}$ shut down the reaction (entry 8). Ethereal solvents are generally suitable other than MTBE (entry 9, 10 vs. 11). Toluene can also be used (entry 12). Highly coordinating DMPU resulted in low yield (entry 13). The best result was obtained by subtle concentration of the reaction mixture (entry 14).

Table S1. Optimization of Reaction Conditions ${ }^{a}$

\begin{tabular}{|c|c|c|c|c|c|}
\hline \multirow[b]{3}{*}{ Entry } & \multirow{3}{*}{${ }_{1 \mathbf{b}}^{\mathrm{O}}$} & \multicolumn{2}{|c|}{$\begin{array}{l}\text { 1) Base (X equiv), }-78^{\circ} \mathrm{C} \text {, } \\
\text { then rt, } 30 \mathrm{~min}\end{array}$} & \multirow{2}{*}{${ }_{\mathrm{pinB}}^{\mathrm{Ph}}$} & \multirow{2}{*}{$\int_{0}^{\mathrm{NH}}$} \\
\hline & & \multicolumn{2}{|c|}{$\begin{array}{l}\text { 2) } \mathrm{Ph}=\text { Bpin 2a (1.5 equiv) } \\
\text { Solvent }(0.13 \mathrm{M}), \text { Temp., } 16 \mathrm{~h}\end{array}$} & & \\
\hline & & $\mathrm{X}$ & Temp. $\left({ }^{\circ} \mathrm{C}\right)$ & Solvent & Yield (\%) \\
\hline 1 & ${ }^{\mathrm{n}} \mathrm{BuLi}$ & 1.0 & 90 & THF & 63 \\
\hline 2 & $\mathrm{NaH}$ & 1.0 & 90 & THF & 74 \\
\hline 3 & $\mathrm{KH}$ & 1.0 & 90 & THF & 56 \\
\hline 4 & none & - & 90 & THF & - \\
\hline 5 & ${ }^{\mathrm{n}} \mathrm{BuLi}$ & 1.1 & 90 & THF & 73 \\
\hline 6 & ${ }^{\mathrm{n}} \mathrm{BuLi}$ & 1.1 & 110 & THF & 88 \\
\hline 7 & ${ }^{\mathrm{n}} \mathrm{BuLi}$ & 1.1 & 70 & THF & 33 \\
\hline 8 & ${ }^{\mathrm{n}} \mathrm{BuLi}$ & 1.1 & $\mathrm{rt}$ & THF & 2 \\
\hline 9 & ${ }^{\mathrm{n}} \mathrm{BuLi}$ & 1.1 & 110 & $\mathrm{DME}$ & 76 \\
\hline 10 & ${ }^{\mathrm{n}} \mathrm{BuLi}$ & 1.1 & 110 & ${ }^{\mathrm{n}} \mathrm{Bu}_{2} \mathrm{O}$ & 64 \\
\hline 11 & ${ }^{\mathrm{n}} \mathrm{BuLi}$ & 1.1 & 110 & MTBE & 3 \\
\hline 12 & ${ }^{\mathrm{n}} \mathrm{BuLi}$ & 1.1 & 110 & Toluene & 54 \\
\hline 13 & ${ }^{\mathrm{n}} \mathrm{BuLi}$ & 1.1 & 110 & DMPU & 13 \\
\hline $14^{b}$ & ${ }^{\mathrm{n}} \mathrm{BuLi}$ & 1.1 & 110 & THF & $90(85 \%)$ \\
\hline
\end{tabular}

${ }^{a}{ }^{1} \mathrm{H}$ NMR yields determined using mesitylene as an internal standard. Isolated yield in parentheses.

${ }^{b} 0.19 \mathrm{M}$. 


\section{2-3. General Procedure for Alkynylboration of Alkynamides}

(E)-N-ethyl-4-phenyl-2-(phenyl(4,4,5,5-tetramethyl-1,3,2-dioxaborolan-2-yl)methylene)but-3-ynamide (3a)

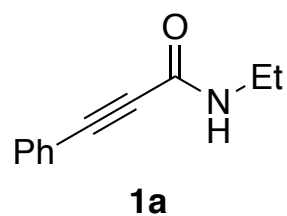

$1 \mathrm{a}$
1) ${ }^{\mathrm{n} B u L i}\left(1.1\right.$ equiv), $-78^{\circ} \mathrm{C}$, then $\mathrm{rt}, 30 \mathrm{~min}$

2) $\mathrm{Ph}=$ Bpin 2a (1.5 equiv)

$\operatorname{THF}(0.19 \mathrm{M}), 110^{\circ} \mathrm{C}, 16 \mathrm{~h}$

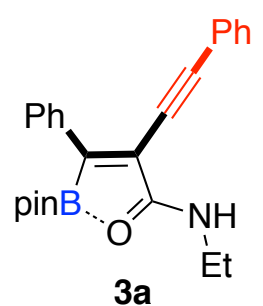

3a

Alkynamide 1a (43.3 mg, $0.25 \mathrm{mmol})$ and anhydrous THF $(1.3 \mathrm{~mL})$ were added to a heat gun-dried greaseless Schlenk tube. To the tube was added ${ }^{\mathrm{n}} \mathrm{BuLi}(2.50 \mathrm{M}$ nexane solution, $0.11 \mathrm{~mL}, 0.275 \mathrm{mmol})$ at $-78^{\circ} \mathrm{C}$ under argon atmosphere, and the mixture was stirred for $30 \mathrm{~min}$ at rt. To the solution was added alkynylboronate $\mathbf{2 a}(95.0 \mathrm{mg}$; calculated based on the purity of the obtained reagent, $0.375 \mathrm{mmol}$ ) at rt, and the reaction vessel was sealed and stirred at $110^{\circ} \mathrm{C}$. After $16 \mathrm{~h}$, the reaction was cooled to $\mathrm{rt}$ and quenched with aqueous $\mathrm{NH}_{4} \mathrm{Cl}$, followed by extraction with EtOAc three times. The combined organic layer was dried over $\mathrm{Na}_{2} \mathrm{SO}_{4}$, filtered, and concentrated in vacuo. Purification by preparative thin-layer chromatography (dichloromethane) afforded the product 3a as a colorless solid in $92 \%$ yield $(92.3 \mathrm{mg}) .{ }^{\mathbf{1}} \mathbf{H}$ NMR (400 MHz, $\left.\mathbf{C D C l}_{3}\right): \delta 1.28(\mathrm{~s}, 12 \mathrm{H}), 1.34(\mathrm{t}, J=7.3 \mathrm{~Hz}, 3 \mathrm{H}), 3.63(\mathrm{dq}, J=6.2,7.3 \mathrm{~Hz}, 2 \mathrm{H}), 6.69(\mathrm{br} \mathrm{t}, J=6.2 \mathrm{~Hz}, 1 \mathrm{H})$, 7.26-7.45 (m, 8H), $7.97(\mathrm{dd}, J=1.4,7.0 \mathrm{~Hz}, 2 \mathrm{H}) .{ }^{11} \mathbf{B}$ NMR (128 $\left.\mathbf{~ M H z}, \mathbf{C D C l}_{3}\right): \delta 15.2 .{ }^{13} \mathbf{C} \mathbf{N M R}(\mathbf{1 0 0} \mathbf{~ M H z}$, $\left.\mathbf{C D C l}_{3}\right): \delta 14.4,25.9,37.1,80.9,81.1,96.6,113.6,122.1,127.8,128.5,128.8,129.0,129.2,131.7,137.6,172.1$. The carbon directly attached to the boron atom was not detected, likely due to quadrupolar relaxation. IR (ATR-IR): 2962, 1636, 1509, 1373, 1117, 1024, 972, 751, 687, 632, $580 \mathrm{~cm}^{-1}$. HRMS (ESI): $m / z$ calculated for $\mathrm{C}_{25} \mathrm{H}_{29} \mathrm{BNO}_{3}{ }^{+}[\mathrm{M}+\mathrm{H}]^{+}=$ 402.2235, found: 402.2234 . mp: $130.8-131.0{ }^{\circ} \mathrm{C}$ (recrystallized from dichloromethane/ ${ }^{\mathrm{n} h e x a n e}$ ). Anal. $(\mathbf{3 a}+\mathbf{5} / \mathbf{4}$ $\mathbf{H}_{2} \mathbf{O}$ ): Calcd: C, 70.85; H, 7.25; N, 3.30. Found: C, 71.07; H, 7.11; N, 3.46. X-ray analysis: CCDC 1886119.

Unless otherwise noted, the following reactions were performed on a $0.25 \mathrm{mmol}$ scale.

\section{Large Scale Reaction:}

Alkynamide $1 \mathrm{a}$ ( $554.3 \mathrm{mg}, 3.2 \mathrm{mmol})$ and anhydrous THF $(16.6 \mathrm{~mL})$ were added to a heat gun-dried greaseless Schlenk tube. To the tube was added ${ }^{n} \mathrm{BuLi}(2.34 \mathrm{M}$ "hexane solution, $1.50 \mathrm{~mL}, 3.52 \mathrm{mmol})$ at $-78{ }^{\circ} \mathrm{C}$ under argon atmosphere, and the mixture was stirred for $30 \mathrm{~min}$ at rt. To the solution was added alkynylboronate $\mathbf{2 a}(1.217 \mathrm{~g}$; calculated based on the purity of the obtained reagent, $4.80 \mathrm{mmol}$ ) at $\mathrm{rt}$, and the reaction vessel was sealed and stirred at $110{ }^{\circ} \mathrm{C}$. After $16 \mathrm{~h}$, the reaction was cooled to $\mathrm{rt}$ and quenched with aqueous $\mathrm{NH}_{4} \mathrm{Cl}$, followed by extraction with EtOAc three times. The combined organic layer was dried over $\mathrm{Na}_{2} \mathrm{SO}_{4}$, filtered, and concentrated in vacuo to give a crude mixture as a yellow solid. This solid was triturated in "hexane $(10 \mathrm{~mL})$ in an ice-water bath, filtered, washed with a small amount of ice-cold "hexane $(\times 3)$, and dried in vacuo to yield pure $3 \mathbf{a}(819.4 \mathrm{mg})$ as a pale yellow solid. The filtrate was concentrated and the residue was purified by MPLC (gradient, dichloromethane/EtOAc $=100 / 0 \rightarrow 53 / 48)$ to give the second crop $(347.9 \mathrm{mg})$. The total yield of pure 3 a was $91 \%(1.167 \mathrm{~g})$. 


\section{2-4. Generality of $N$-substituents (Scheme 2)}

(E)- $N$-methyl-4-phenyl-2-(phenyl(4,4,5,5-tetramethyl-1,3,2-dioxaborolan-2-yl)methylene)but-3-ynamide (3b)

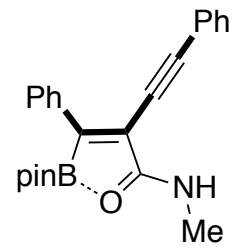

Following the General Procedure (pTLC: chloroform $\rightarrow$ dichloromethane/EtOAc 85\%), the titled compound was obtained as a colorless solid in $85 \%(82.1 \mathrm{mg}) .{ }^{1} \mathbf{H}$ NMR (400 $\left.\mathbf{M H z}, \mathbf{C D C l}_{3}\right): \delta 1.29$ (s, $12 \mathrm{H}), 3.18(\mathrm{~d}, J=5.2 \mathrm{~Hz}, 3 \mathrm{H}), 6.76(\mathrm{br} \mathrm{s}, 1 \mathrm{H}), 7.26-7.45(\mathrm{~m}, 8 \mathrm{H}), 7.97(\mathrm{~d}, J=7.2 \mathrm{~Hz}, 2 \mathrm{H}) .{ }^{11} \mathbf{B} \mathbf{~ N M R}$ (128 MHz, $\mathbf{C D C l} 3$ ): $\delta 14.7 .{ }^{13} \mathbf{C}$ NMR (100 $\mathbf{~ M H z , ~} \mathbf{C D C l}_{3}$ ): $\delta 25.9,28.4,81.0,81.1,96.6,113.6,122.1$, $127.8,128.5,128.8,129.1,129.3,131.7,137.6,172.9$. The carbon directly attached to the boron atom

was not detected, likely due to quadrupolar relaxation. IR (ATR-IR): 3379, 2970, 1661, 1509, 1492, 1442, 1363, 1312, 1116, 1066, 1030, 974, 939, 912, 867, 793, 756, 720, 688, 632, 573, 528, $506 \mathrm{~cm}^{-1}$. HRMS (ESI): $\mathrm{m} / z$ calculated for $\mathrm{C}_{24} \mathrm{H}_{27} \mathrm{BNO}_{3}{ }^{+}[\mathrm{M}+\mathrm{H}]^{+}=\quad 388.2079$, found: 388.2080. mp: 185.5-186.5 ${ }^{\circ} \mathrm{C}$ (recrystallized from dichloromethane/ ${ }^{\mathrm{n}}$ hexane). Anal. $\left(\mathbf{3 b}+\mathbf{6} / \mathbf{5} \mathbf{H}_{2} \mathbf{O}\right)$ : Calcd: C, 70.50; H, 7.00; N, 3.43. Found: C, 70.73; H, 6.86; N, 3.61.

(E)-N-isobutyl-4-phenyl-2-(phenyl(4,4,5,5-tetramethyl-1,3,2-dioxaborolan-2-yl)methylene)but-3-ynamide (3c)

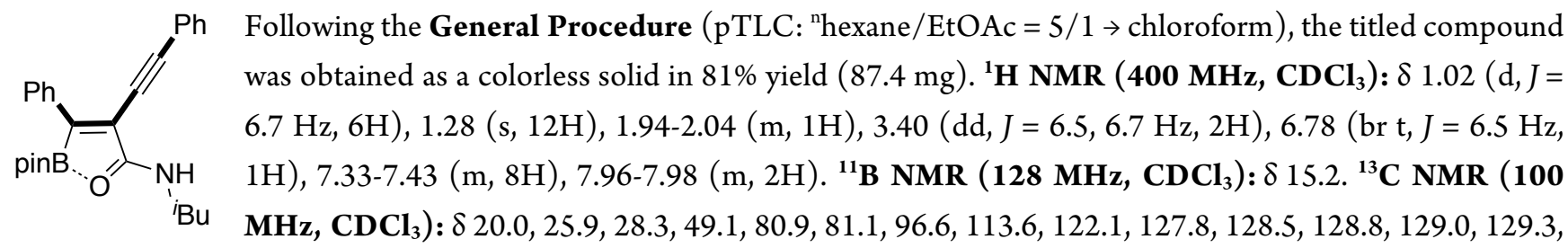

131.6, 137.6, 172.3. The carbon directly attached to the boron atom was not detected, likely due to quadrupolar relaxation. IR (ATR-IR): 2969, 2929, 2871, 1736, 1635, 1507, 1365, 1120, 1027, 971, 935, 851, 753, 688, 616, $507 \mathrm{~cm}^{-1}$. HRMS (ESI): $m / z$ calculated for $\mathrm{C}_{27} \mathrm{H}_{33} \mathrm{BNO}_{3}{ }^{+}[\mathrm{M}+\mathrm{H}]^{+}=430.2548$, found: 430.2549 . mp: $69.2-72.3{ }^{\circ} \mathrm{C}$ (recrystallized from dichloromethane $/{ }^{n}$ hexane). Anal. $\left(3 \mathbf{c}+\mathbf{H}_{2} \mathbf{O}\right)$ : Calcd: C, 72.49; H, 7.66; N, 3.13. Found: C, 72.35; H, 7.73; N, 3.15 .

(E)-N-benzyl-4-phenyl-2-(phenyl(4,4,5,5-tetramethyl-1,3,2-dioxaborolan-2-yl)methylene)but-3-ynamide (3d)

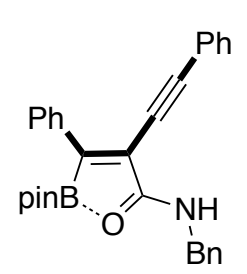

Following the General Procedure (pTLC: ${ }^{\text {nhexane } / E t O A c}=5 / 1 \rightarrow$ chloroform), the titled compound was obtained as a colorless solid in 91\% yield (105.8 mg). ${ }^{1} \mathbf{H}$ NMR (400 $\left.\mathbf{~ M H z}, \mathbf{C D C l}_{3}\right): \delta 1.31$ (s, $12 \mathrm{H}), 4.75(\mathrm{~d}, J=5.9 \mathrm{~Hz}, 2 \mathrm{H}), 6.98(\mathrm{br} \mathrm{t}, J=5.9 \mathrm{~Hz}, 1 \mathrm{H}), 7.26-7.43(\mathrm{~m}, 13 \mathrm{H}), 7.98(\mathrm{~d}, J=7.0 \mathrm{~Hz}, 2 \mathrm{H})$. ${ }^{11}$ B NMR (128 MHz, $\left.\mathbf{C D C l}_{3}\right): \delta 15.5 .{ }^{13} \mathbf{C}$ NMR (100 $\left.\mathbf{M H z}, \mathbf{C D C l}_{3}\right): \delta 25.9,46.0,81.0,81.1,96.8$, $113.8,122.0,127.8,128.1,128.5,128.5,128.9,129.0,129.2,129.4,131.6,135.6,137.5,172.1$. The carbon directly attached to the boron atom was not detected, likely due to quadrupolar relaxation. IR (ATR-IR): 3249, 2978, 1737, 1633, 1508, 1494, 1429, 1365, 1179, 1139, 1119, 1027, 970, 937, 863, 753, 691, 608, 572, $526 \mathrm{~cm}^{-1}$. HRMS (ESI): $\mathrm{m} / z$ calculated for $\mathrm{C}_{24} \mathrm{H}_{27} \mathrm{BNO}_{3}{ }^{+}[\mathrm{M}+\mathrm{H}]^{+}=464.2392$, found: 464.2394 . mp: $79.5-80.8{ }^{\circ} \mathrm{C}$

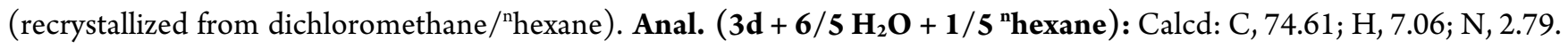
Found: C, 74.73; H, 7.11; N, 2.93. 
(E)- $\mathrm{N}$-isopropyl-4-phenyl-2-(phenyl(4,4,5,5-tetramethyl-1,3,2-dioxaborolan-2-yl)methylene)but-3-ynamide (3e)

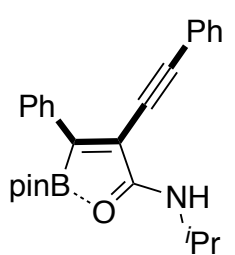

Following the General Procedure (pTLC: ${ }^{\text {nhexane }} / \mathrm{EtOAc}=5 / 1 \rightarrow$ chloroform), the titled compound was obtained as a colorless solid in 82\% yield (84.9 mg). ${ }^{1} \mathbf{H}$ NMR (400 $\left.\mathbf{~ M H z}, \mathbf{C D C l}_{3}\right): \delta 1.28(\mathrm{~s}, 12 \mathrm{H})$, $1.35(\mathrm{~d}, J=6.6 \mathrm{~Hz}, 6 \mathrm{H}), 4.26-4.35(\mathrm{~m}, 1 \mathrm{H}), 6.49(\mathrm{br} \mathrm{d}, J=7.8 \mathrm{~Hz}, 1 \mathrm{H}), 7.32-7.45(\mathrm{~m}, 8 \mathrm{H}), 7.96(\mathrm{~d}, J=$ $7.1 \mathrm{~Hz}, 2 \mathrm{H}) .{ }^{11} \mathbf{B}$ NMR (128 $\left.\mathbf{~ M H z}, \mathbf{C D C l}_{3}\right): \delta 14.9 .{ }^{13} \mathbf{C} \mathbf{N M R}\left(\mathbf{1 0 0} \mathbf{~ M H z}, \mathbf{C D C l}_{3}\right): \delta 22.3,25.9,44.8$, $80.9,81.0,96.6,113.7,122.1,127.8,128.5,128.8,129.0,129.2,131.6,137.7,171.3$. The carbon directly attached to the boron atom was not detected, likely due to quadrupolar relaxation. IR (ATR-IR): 2968, 1737, 1634, 1364, 1120, 1028, 970, 920, 753, 689, 527, $507 \mathrm{~cm}^{-1}$. HRMS (ESI): $m / z$ calculated for $\mathrm{C}_{26} \mathrm{H}_{31} \mathrm{BNO}_{3}{ }^{+}[\mathrm{M}+\mathrm{H}]^{+}=\quad 416.2392$, found: 416.2393. mp: 68.4-69.4 ${ }^{\circ} \mathrm{C}$ (recrystallized from

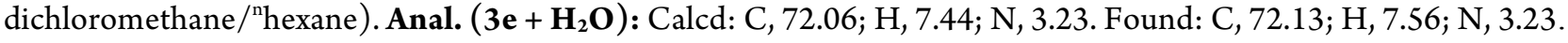

(E)-N-cyclopropyl-4-phenyl-2-(phenyl(4,4,5,5-tetramethyl-1,3,2-dioxaborolan-2-yl)methylene)but-3-ynamide (3f)

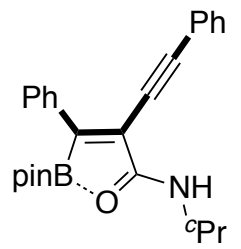

Following the General Procedure (Crude mixture was triturated in "hexane $(10 \mathrm{~mL})$ in an ice-water bath, filtered, washed with a small amount of ice-cold "hexane ( $\times 3)$, and dried in vacuo.), the titled compound was obtained as a yellowish solid in $84 \%$ yield $(87.1 \mathrm{mg}) .{ }^{1} \mathbf{H}$ NMR $\left(400 \mathbf{M H z}, \mathbf{C D C l}_{3}\right): \delta$ 0.81-0.85 (m, 2H), 0.93-0.98 (m, 2H), $1.29(\mathrm{~s}, 12 \mathrm{H}), 3.05$ (ddd, $J=3.8,7.5,14.8 \mathrm{~Hz}, 1 \mathrm{H}), 6.73$ (br s, $1 \mathrm{H}), 7.32-7.43(\mathrm{~m}, 8 \mathrm{H}), 7.96(\mathrm{~d}, J=7.0 \mathrm{~Hz}, 2 \mathrm{H}) .{ }^{11} \mathbf{B}$ NMR $\left(128 \mathbf{M H z} \mathbf{C D C l}_{3}\right): \delta 15.9 .{ }^{13} \mathbf{C} \mathbf{~ N M R}$ (100 MHz, $\left.\mathbf{C D C l}_{3}\right)$ : $\delta$ 6.9, 24.6, 25.9, 81.0, 81.0, 96.6, 113.6, 122.0, 127.8, 128.5, 128.8, 129.1, 129.3, 131.6, 137.6, 173.6. The carbon directly attached to the boron atom was not detected, likely due to quadrupolar relaxation. IR (ATR-IR): 2970, 2370, 1737, 1635, 1522, 1364, 1217, 1120, 1029, 970, 921, 853, 751, 688, 529, $507 \mathrm{~cm}^{-1}$. HRMS (ESI): $m / z$ calculated for $\mathrm{C}_{26} \mathrm{H}_{28} \mathrm{BNNaO}_{3}{ }^{+}[\mathrm{M}+\mathrm{Na}]^{+}=436.2054$, found: 436.2054 . mp: $80.2-81.4{ }^{\circ} \mathrm{C}$ (recrystallized from dichloromethane $/{ }^{n}$ hexane). Anal. (3f $+\mathbf{H}_{\mathbf{2}} \mathbf{O}$ ): Calcd: C, 72.40; H, 7.01; N, 3.25. Found: C, 72.37; H, 7.11; N, 3.25.

(E)-N-(tert-butyl)-4-phenyl-2-(phenyl(4,4,5,5-tetramethyl-1,3,2-dioxaborolan-2-yl)methylene)but-3-ynamide $(3 g)$

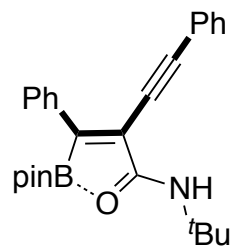

Following the General Procedure (pTLC: dichloromethane), the titled compound was obtained as colorless sticky oil in 16\% yield (17.5 mg). ${ }^{1} \mathbf{H}$ NMR (400 $\left.\mathbf{M H z}, \mathbf{C D C l}_{3}\right): \delta 1.28(\mathrm{~s}, 12 \mathrm{H}), 1.53(\mathrm{~s}, 9 \mathrm{H})$, $6.63(\mathrm{br} \mathrm{s}, 1 \mathrm{H}), 7.26-7.43(\mathrm{~m}, 8 \mathrm{H}), 7.95(\mathrm{~d}, J=6.8 \mathrm{~Hz}, 2 \mathrm{H}) .{ }^{11} \mathbf{B}$ NMR $\left(\mathbf{1 2 8} \mathbf{~ M H z}, \mathbf{C D C l}_{3}\right): \delta 15.4 .{ }^{13} \mathbf{C}$ NMR (100 MHz, $\mathbf{C D C l}_{3}$ ): $\delta 25.9,28.7,54.1,80.7,81.2,96.7,114.2,122.1,127.7,128.5,128.8,129.0$,

$129.1,131.6,137.7,171.3$. The carbon directly attached to the boron atom was not detected, likely due

to quadrupolar relaxation. IR (ATR-IR): 3380, 2267, 1633, 1532, 1444, 1369, 13137, 1180, 1128, 1050, 972, 745, 688, 662, 611, 575, $532 \mathrm{~cm}^{-1}$. HRMS (ESI): $m / z$ calculated for $\mathrm{C}_{27} \mathrm{H}_{33} \mathrm{BNO}_{3}{ }^{+}[\mathrm{M}+\mathrm{H}]^{+}=430.2548$, found: 430.2548. Anal. $(3 \mathrm{~g}+13 / 10$ chloroform ): Calcd: C, 58.15; H, 5.74; N, 2.40. Found: C, 57.88; H, 5.98; N, 2.49. Elemental analysis was conducted after purification using the recycling preparative HPLC. 
(E)-4-phenyl-2-(phenyl(4,4,5,5-tetramethyl-1,3,2-dioxaborolan-2-yl)methylene)- $N$-(2-((triisopropylsilyl)oxy)e thyl)but-3-ynamide (3i)

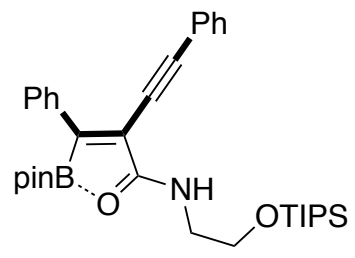

Following the General Procedure (pTLC: " hexane/EtOAc $=5 / 1$ ), the titled compound was obtained as a colorless solid in 85\% yield (122.2 mg). ${ }^{1} \mathbf{H}$ NMR (400 $\left.\mathbf{~ M H z}, \mathbf{C D C l}_{3}\right): \delta 1.02$ $(\mathrm{d}, J=5.9 \mathrm{~Hz}, 18 \mathrm{H}), 1.01-1.11(\mathrm{~m}, 3 \mathrm{H}), 1.28(\mathrm{~s}, 12 \mathrm{H}), 3.72(\mathrm{td}, J=5.2,5.3 \mathrm{~Hz}, 2 \mathrm{H}), 3.91$ $(\mathrm{t}, J=5.2 \mathrm{~Hz}, 2 \mathrm{H}), 7.29-7.41(\mathrm{~m}, 9 \mathrm{H}), 7.97(\mathrm{dd}, J=1.3,7.0 \mathrm{~Hz}, 2 \mathrm{H}) .{ }^{11} \mathbf{B}$ NMR (128 MHz, $\left.\mathbf{C D C l}_{3}\right): \delta 17.2 .{ }^{13} \mathbf{C}$ NMR (100 MHz, $\left.\mathbf{C D C l}_{3}\right): \delta 11.8,17.9,25.9,43.9,61.2,80.7,80.9,96.4$, $113.8,122.1,127.8,128.3,128.8,128.9,129.2,131.7,137.6,172.1$. The carbon directly attached to the boron atom was not detected, likely due to quadrupolar relaxation. IR (ATR-IR): 3361, 2943, 2865, 1737, 1637, 1516, 1459, 1363, 1180, 1119, 1070, 972, 917, 882, 755, 687, 663, $529 \mathrm{~cm}^{-1}$. HRMS (ESI): $m / z$ calculated for $\mathrm{C}_{34} \mathrm{H}_{49} \mathrm{BNO}_{4} \mathrm{Si}^{+}[\mathrm{M}+\mathrm{H}]^{+}=$ 574.3518, found: 574.3518. mp: $67.5-69.6{ }^{\circ} \mathrm{C}$ (recrystallized from dichloromethane/ ${ }^{\mathrm{n}}$ hexane). Anal. $(\mathbf{3 i}+\mathbf{6} / \mathbf{5}$ $\mathbf{H}_{2} \mathbf{O}$ ): Calcd: C, 68.60; H, 8.53; N, 2.35. Found: C, 68.67; H, 8.39; N, 2.38 .

(E)-N-allyl-4-phenyl-2-(phenyl(4,4,5,5-tetramethyl-1,3,2-dioxaborolan-2-yl)methylene)but-3-ynamide (3j)

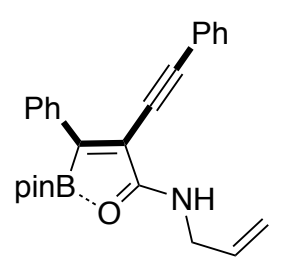

Following the General Procedure (1.0 eq. of ${ }^{\mathrm{n}} \mathrm{BuLi}$ was used. MPLC: gradient, dichloromethane $/$ EtOAc $=100 / 0 \rightarrow 61 / 39)$, the titled compound was obtained as a off-white solid in $81 \%$ yield $(83.7 \mathrm{mg}) .{ }^{1} \mathbf{H}$ NMR (400 $\left.\mathbf{~ M H z}, \mathbf{C D C l}_{3}\right): \delta 1.29(\mathrm{~s}, 12 \mathrm{H}), 4.19(\mathrm{dd}, J=6.0,6.0 \mathrm{~Hz}$, $2 \mathrm{H}), 5.31(\mathrm{~d}, J=10.3 \mathrm{~Hz}, 1 \mathrm{H}), 5.36(\mathrm{~d}, J=17.0 \mathrm{~Hz}, 1 \mathrm{H}), 5.94(\mathrm{ddt}, J=17.0,10.3,6.0 \mathrm{~Hz}, 1 \mathrm{H}), 6.75$ (br t, $J=6.0 \mathrm{~Hz}, 1 \mathrm{H}), 7.26-7.44(\mathrm{~m}, 8 \mathrm{H}), 7.97(\mathrm{dd}, J=8.4,1.4 \mathrm{~Hz}, 2 \mathrm{H}) .{ }^{11} \mathbf{B}$ NMR (128 MHz, $\left.\mathbf{C D C l}_{3}\right): \delta 15.4 .{ }^{13} \mathbf{C}$ NMR (100 $\left.\mathbf{~ M H z}, \mathbf{C D C l}_{3}\right): \delta 25.9,44.3,81.0,81.1,96.7,113.8,119.1,122.0,127.8,128.5,128.8$, $129.1,129.3,131.7,131.7,137.5,172.0$. The carbon directly attached to the boron atom was not detected, likely due to quadrupolar relaxation. IR (ATR-IR): 2970, 1737, 1634, 1522, 1364, 1318, 1201, 1123, 971, 914, 755, 689, $558 \mathrm{~cm}^{-1}$. HRMS (ESI): $m / z$ calculated for $\mathrm{C}_{26} \mathrm{H}_{28} \mathrm{BNNaO}_{3}{ }^{+}[\mathrm{M}+\mathrm{Na}]^{+}=436.2054$, found: 436.2054. mp: $109.8-111.5{ }^{\circ} \mathrm{C}$

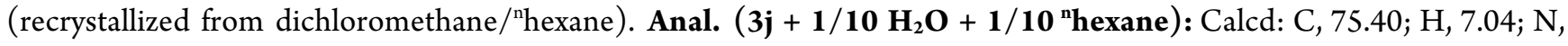
3.31. Found: C, 75.24; H, 6.90; N, 3.41.

(E)-4-phenyl-2-(phenyl(4,4,5,5-tetramethyl-1,3,2-dioxaborolan-2-yl)methylene)- $\mathrm{N}$-(prop-2-yn-1-yl)but-3-ynam ide (3k)

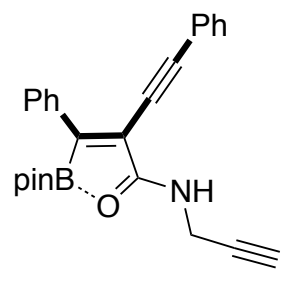

Following the General Procedure ( $1 \mathrm{mmol}$ scale. 1.0 eq. of ${ }^{n} \mathrm{BuLi}$ was used. MPLC: gradient, dichloromethane $/$ EtOAc $=100 / 0 \rightarrow 52 / 48)$, the titled compound was obtained as a yellow solid in 47\% yield (195.3 mg). ${ }^{1} \mathbf{H}$ NMR (400 $\left.\mathbf{~ M H z}, \mathbf{C D C l}_{3}\right): \delta 1.30(\mathrm{~s}, 12 \mathrm{H}), 2.39(\mathrm{t}, J=2.5 \mathrm{~Hz}, 1 \mathrm{H}), 4.36$ $(\mathrm{dd}, J=2.5,5.3 \mathrm{~Hz}, 2 \mathrm{H}), 6.84(\mathrm{br} \mathrm{t}, J=5.3 \mathrm{~Hz}, 1 \mathrm{H}), 7.34-7.46(\mathrm{~m}, 8 \mathrm{H}), 7.95(\mathrm{~d}, J=7.0 \mathrm{~Hz}, 2 \mathrm{H})$. ${ }^{11}$ B NMR (128 MHz, $\left.\mathbf{C D C l}_{3}\right): \delta 17.5 .{ }^{13} \mathbf{C}$ NMR (100 MHz, $\left.\mathbf{C D C l}_{3}\right): \delta 25.8,31.5,73.8,81.1,81.5$, $81.5,96.9,114.2,122.0,127.9,128.5,128.8,129.1,129.4,131.7,137.4,171.2$. The carbon directly attached to the boron atom was not detected, likely due to quadrupolar relaxation. IR (ATR-IR): 3283, 2970, 2926, 2852, $1738,1637,1506,1443,1366,1311,1176,1124,1029,1003,970,938,862,756,726,691,616,587,527,507 \mathrm{~cm}^{-1}$. HRMS (ESI): $m / z$ calculated for $\mathrm{C}_{26} \mathrm{H}_{27} \mathrm{BNO}_{3}{ }^{+}[\mathrm{M}+\mathrm{H}]^{+}=412.2079$, found: 412.2079. mp: $161.6-162.4{ }^{\circ} \mathrm{C}$

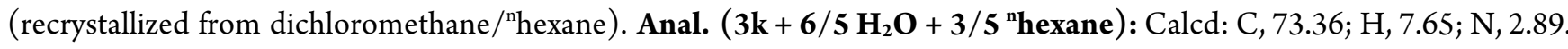
Found: C, 73.37; H, 7.37; N, 3.12. 

mide (31)

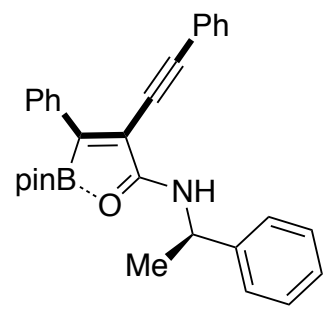

Following the General Procedure (1.0 eq. of ${ }^{\mathrm{n}} \mathrm{BuLi}$ was used. MPLC: gradient, dichloromethane $/$ EtOAc $=100 / 0 \rightarrow 63 / 37)$, the titled compound was obtained as a yellowish form in $72 \%$ yield $\left(86.0 \mathrm{mg}\right.$ ) after further purification using the recycling preparative HPLC. ${ }^{1} \mathbf{H}$ NMR (400 MHz, $\left.\mathbf{C D C l}_{3}\right): \delta 1.28(\mathrm{~s}, 6 \mathrm{H}), 1.30(\mathrm{~s}, 6 \mathrm{H}), 1.71(\mathrm{~d}, J=6.8 \mathrm{~Hz}, 3 \mathrm{H}), 5.31(\mathrm{dq}, J=$ 7.6, $6.8 \mathrm{~Hz}, 1 \mathrm{H}), 6.88(\mathrm{br} \mathrm{d}, J=7.6 \mathrm{~Hz}, 1 \mathrm{H}), 7.31-7.43(\mathrm{~m}, 13 \mathrm{H}), 7.97(\mathrm{~d}, J=7.7 \mathrm{~Hz}, 2 \mathrm{H}) .{ }^{11} \mathbf{B}$ NMR (128 MHz, $\left.\mathbf{C D C l}_{3}\right): \delta 15.5 .{ }^{13} \mathbf{C}$ NMR (100 $\left.\mathbf{~ M H z , ~} \mathbf{C D C l}_{3}\right): \delta 21.0,25.8,25.8,51.7,80.9$ (2C), 81.0, 96.7, 113.7, 121.9, 126.2, 127.7, 128.2, 128.4, 128.7, 129.0, 129.0, 129.2, 131.5, 137.5, 140.5, 171.1. The carbon directly attached to the boron atom was not detected, likely due to quadrupolar relaxation. IR (ATR-IR): 3389, 3059, 2970, 2925, 2224, 1626, 1576, 1523, 1494, 1443, 1384, 1363, 1327, 1239, 1187, 1137, 1070, 1030, 973, 913, 861, 755, 730, 696, 596, 574, $532 \mathrm{~cm}^{-1}$. HRMS (ESI): $\mathrm{m} / z$ calculated for $\mathrm{C}_{31} \mathrm{H}_{32} \mathrm{BNNaO}_{3}{ }^{+}[\mathrm{M}+\mathrm{Na}]^{+}=500.2367$, found: 500.2377. mp: $139.1-140.7^{\circ} \mathrm{C}$ (recrystallized from diethylether/nhexane). Anal. (31 + 1/3 $\mathbf{H}_{2} \mathbf{O}$ ): Calcd: C, $77.02 ; \mathrm{H}$, 6.81; N, 2.90. Found: C, 76.98; H, 6.82; N, 2.83. HPLC: CHIRALPAK IA-3 column, " $1.0 \mathrm{~mL} / \mathrm{min}, \mathrm{T}_{\mathrm{r}} 5.40 \mathrm{~min}$ (minor), 7.41 (major), e.r. $=1.8: 98.2 .[\boldsymbol{\alpha}]_{\mathrm{D}}{ }^{20}:+11.8$ (c 0.20 , chloroform).

\section{HPLC charts:}

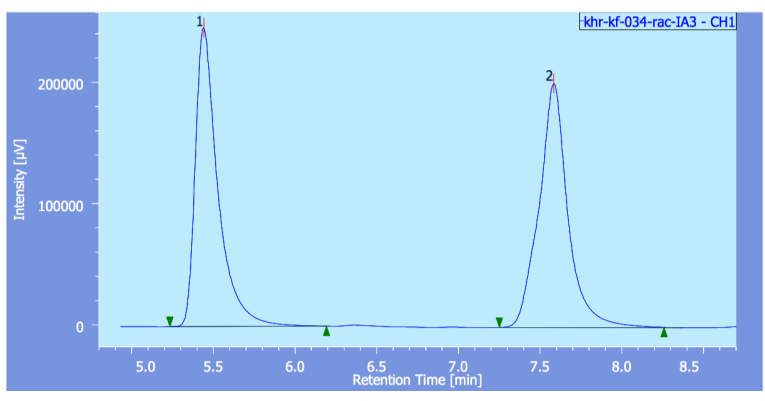

racemate

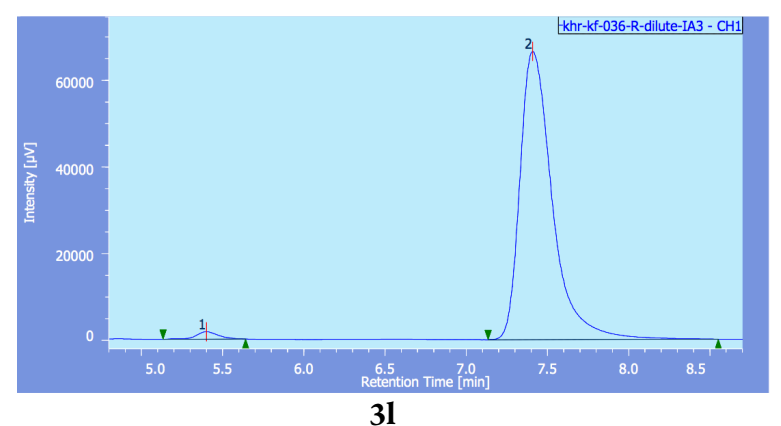

31

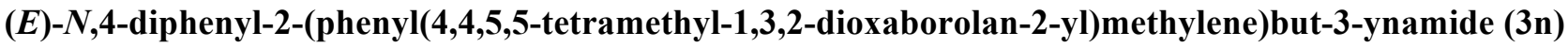

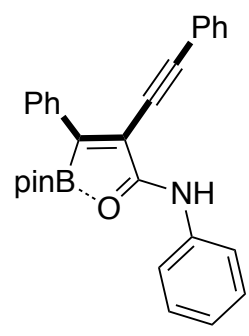

Following the General Procedure (Crude mixture was triturated in ${ }^{\mathrm{n}}$ hexane $(10 \mathrm{~mL})$ in an ice-water bath, filtered, washed with a small amount of ice-cold nexane ( $\times 3)$, and dried in vacuo.), the titled compound was obtained as a colorless solid in $79 \%$ yield $(88.6 \mathrm{mg}) .{ }^{1} \mathbf{H}$ NMR (400 $\left.\mathbf{~ M H z}, \mathbf{C D C l}_{3}\right): \delta$ $1.34(\mathrm{~s}, 12 \mathrm{H}), 7.24-7.28(\mathrm{~m}, 1 \mathrm{H}), 7.36-7.44(\mathrm{~m}, 8 \mathrm{H}), 7.48-7.50(\mathrm{~m}, 2 \mathrm{H}), 7.64(\mathrm{~d}, J=7.7 \mathrm{~Hz}, 2 \mathrm{H})$, $8.01(\mathrm{dd}, J=8.4,1.5 \mathrm{~Hz}, 2 \mathrm{H}), 8.39$ (br s, $1 \mathrm{H}) .{ }^{11} \mathbf{B}$ NMR (128 $\left.\mathbf{~ M H z}, \mathbf{C D C l}_{3}\right): \delta 17.8 .{ }^{13} \mathbf{C} \mathbf{~ N M R}(100$ MHz, $\left.\mathbf{C D C l}_{3}\right): \delta 25.8,81.4,81.7,97.6,115.4,120.9,121.9,126.4,128.0,128.6,128.9,129.3,129.3$, $129.6,131.7,135.6,137.5,169.0$. The carbon directly attached to the boron atom was not detected, likely due to quadrupolar relaxation. IR (ATR-IR): 2970, 2925, 2857, 2328, 1738, 1619, 1576, 1519, 1491, 1455, 1356, 1217, 1127, 1031, 973, 939, 865, 760, 749, 687, 625, 553, $516 \mathrm{~cm}^{-1}$. HRMS (ESI): $\mathrm{m} / z$ calculated for $\mathrm{C}_{29} \mathrm{H}_{29} \mathrm{BNO}_{3}{ }^{+}[\mathrm{M}+\mathrm{H}]^{+}=450.2235$, found: 450.2236. mp: 84.3-85.7 ${ }^{\circ} \mathrm{C} \quad$ (recrystallized from dichloromethane/"nhexane). Anal. (3n + 3/2 H2 $\mathbf{O})$ : Calcd: C, 73.12; H, 6.56; N, 2.94. Found: C, 72.90; H, 6.69; N, 3.10. 
(E)-N-(4-methoxyphenyl)-4-phenyl-2-(phenyl(4,4,5,5-tetramethyl-1,3,2-dioxaborolan-2-yl)methylene)but-3-yn amide (3o)

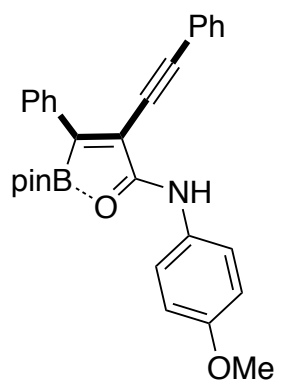

Following the General Procedure, the titled compound was obtained as a yellow solid in $63 \%$ yield (75.8 mg) after purification using the recycling preparative HPLC. ${ }^{1} \mathbf{H}$ NMR (400 $\left.\mathbf{~ M H z}, \mathbf{C D C l}_{3}\right): \delta$ $1.33(\mathrm{~s}, 12 \mathrm{H}), 3.84(\mathrm{~s}, 3 \mathrm{H}), 6.94(\mathrm{dd}, J=2.0,7.0 \mathrm{~Hz}, 2 \mathrm{H}), 7.36-7.44(\mathrm{~m}, 6 \mathrm{H}), 7.47-7.50(\mathrm{~m}, 2 \mathrm{H})$, $7.56(\mathrm{dd}, J=2.0,7.0 \mathrm{~Hz}, 2 \mathrm{H}), 8.00(\mathrm{dd}, J=1.5,8.4 \mathrm{~Hz}, 2 \mathrm{H}) .{ }^{11} \mathbf{B}$ NMR $\left(128 \mathbf{M H z}, \mathbf{C D C l}_{3}\right): \delta 16.5$. ${ }^{13}$ C NMR (100 MHz, $\left.\mathbf{C D C l}_{3}\right): \delta 25.9,55.5,81.2,81.4,97.5,114.4,114.9,121.9,122.5,127.9,128.5$, $128.6,128.9,129.2,129.5,131.7,137.6,157.9,169.0$. The carbon directly attached to the boron atom 1588, 1509, 1424, 1363, 1274, 1245, 1176, 1125, 1031, 970, 941, 866, 828, 803, 770, 755, 691, 630, $516 \mathrm{~cm}^{-1}$. HRMS (ESI): $m / z$ calculated for $\mathrm{C}_{30} \mathrm{H}_{31} \mathrm{BNO}_{4}{ }^{+}[\mathrm{M}+\mathrm{H}]^{+}=480.2341$, found: 480.2341 . mp: $94.3-94.6{ }^{\circ} \mathrm{C}$ (recrystallized from dichloromethane/"nhexane). Anal. (3o + 6/5 H2 $\mathbf{O})$ : Calcd: C, 71.92; H, 6.52; N, 2.80. Found: C, 71.90; H, 6.69; N, 2.93.

(E)-4-phenyl-2-(phenyl(4,4,5,5-tetramethyl-1,3,2-dioxaborolan-2-yl)methylene)- $\mathrm{N}$-(4-(trifluoromethyl)phenyl but-3-ynamide (3p)

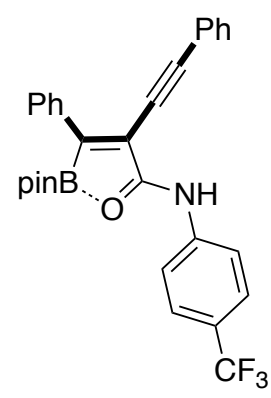

Following the General Procedure (pTLC: dichloromethane), the titled compound was obtained as a yellow solid in 12\% yield (15.8 mg) ${ }^{1} \mathbf{H}$ NMR (400 $\left.\mathbf{~ M H z}, \mathbf{C D C l}_{3}\right): \delta 1.37(\mathrm{~s}, 12 \mathrm{H}), 7.37-7.50(\mathrm{~m}$, $8 \mathrm{H}), 7.68(\mathrm{~d}, J=8.5 \mathrm{~Hz}, 2 \mathrm{H}), 7.78(\mathrm{~d}, J=8.5 \mathrm{~Hz}, 2 \mathrm{H}), 7.96(\mathrm{dd}, J=1.3,8.2 \mathrm{~Hz}, 2 \mathrm{H}) .{ }^{11} \mathbf{B}$ NMR $(128$ MHz, $\left.\mathbf{C D C l}_{3}\right): \delta 17.8 .{ }^{13} \mathbf{C}$ NMR (100 MHz, $\left.\mathbf{C D C l}_{3}\right): \delta 25.6,81.7,82.4,97.9,116.4,120.5,121.7$, $123.8(\mathrm{q}, J=270.1 \mathrm{~Hz}), 126.6(\mathrm{q}, J=3.7 \mathrm{~Hz}), 127.6,127.8(\mathrm{q}, J=32.7 \mathrm{~Hz}), 128.6,128.9,129.5$, $129.7,131.7,137.4,139.0,168.2$. The carbon directly attached to the boron atom was not detected, likely due to quadrupolar relaxation. ${ }^{19} \mathbf{F}$ NMR (376 $\mathbf{M H z}, \mathbf{C D C l}_{3}$ ): $\delta$-62.33. IR (ATR-IR): 2970, $2373,2327,1738,1613,1573,1520,1495,1422,1364,1320,1165,1045,973,938,842,771,754$, 721, 689, 618, 562, 518, $505 \mathrm{~cm}^{-1}$. HRMS (ESI): $m / z$ calculated for $\mathrm{C}_{30} \mathrm{H}_{28} \mathrm{BF}_{3} \mathrm{NO}_{3}{ }^{+}[\mathrm{M}+\mathrm{H}]^{+}=518.2109$, found: 518.2109. mp: $197.0-197.2{ }^{\circ} \mathrm{C}$ (recrystallized from dichloromethane/ ${ }^{\mathrm{n} h e x a n e}$ ). Anal. (3p + $\left.\mathbf{H}_{2} \mathbf{O}\right)$ : Calcd: C, 67.30; H, 5.46; N, 2.62. Found: C, 67.18; H, 5.47; N, 2.73 .

(E)-N-mesityl-4-phenyl-2-(phenyl(4,4,5,5-tetramethyl-1,3,2-dioxaborolan-2-yl)methylene)but-3-ynamide (3q)

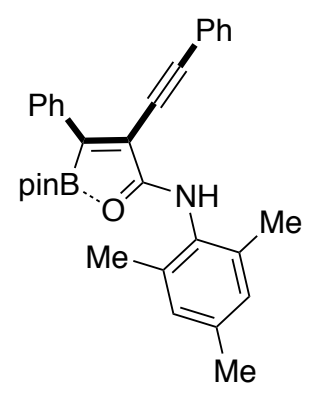

Following the General Procedure (MPLC: gradient, dichloromethane/EtOAc $=100 / 0 \rightarrow 95 / 5$ ), the titled compound was obtained as a pale brown solid in $85 \%$ yield $(105.0 \mathrm{mg})$. ${ }^{1} \mathbf{H}$ NMR (400 MHz, $\left.\mathbf{C D C l}_{3}\right): \delta 1.23(\mathrm{~s}, 12 \mathrm{H}), 2.30(\mathrm{~s}, 6 \mathrm{H}), 2.32(\mathrm{~s}, 3 \mathrm{H}), 6.95(\mathrm{~s}, 2 \mathrm{H}), 7.33-7.46(\mathrm{~m}, 8 \mathrm{H}), 8.01$ $(\mathrm{d}, J=6.8 \mathrm{~Hz}, 2 \mathrm{H}), 8.01-8.03(\mathrm{~m}, 1 \mathrm{H}) .{ }^{11} \mathbf{B}$ NMR (128 $\left.\mathbf{M H z} \mathbf{C D C l}_{3}\right): \delta 16.2 .{ }^{13} \mathbf{C}$ NMR (100 MHz, $\left.\mathbf{C D C l}_{3}\right): \delta 18.6,21.0,25.9,81.3,81.5,97.2,114.2,122.0,127.8,128.6,128.9,129.2,129.2$, $129.3,129.4,131.6,134.3,137.5,138.1,170.2$. The carbon directly attached to the boron atom was not detected, likely due to quadrupolar relaxation. IR (ATR-IR): 3649, 2970, 2924, 2358, $2328,1737,1617,1597,1558,1506,1364,1291,1214,1129,1029,972,933,842,756,710,693$,

594, 569, $530 \mathrm{~cm}^{-1}$. HRMS (ESI): $\mathrm{m} / z$ calculated for $\mathrm{C}_{32} \mathrm{H}_{35} \mathrm{BNO}_{3}{ }^{+}[\mathrm{M}+\mathrm{H}]^{+}=492.2705$, found: 492.2703. mp:

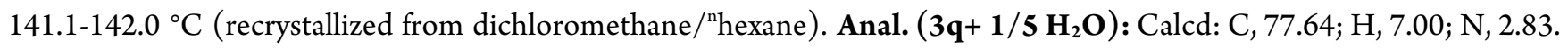
Found: C, 77.60; H, 7.18; N, 2.82 . 


\section{2-5. Scope of Acetylenic Terminus (Scheme 3)}

(E)-N-ethyl-2-(naphthalen-1-yl(4,4,5,5-tetramethyl-1,3,2-dioxaborolan-2-yl)methylene)-4-phenylbut-3-ynamid e (5a)

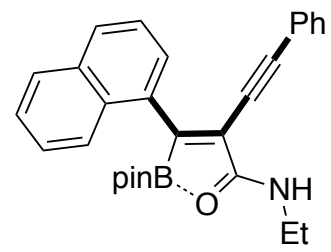

Following the General Procedure (MPLC: gradient, dichloromethane $/ \mathrm{EtOAc}=100 / 0 \rightarrow$ $77 / 23)$, the titled compound was obtained as a yellow solid in $95 \%$ yield $(107.4 \mathrm{mg}) .{ }^{1} \mathbf{H}$ NMR (400 MHz, $\left.\mathbf{C D C l}_{3}\right): \delta 1.10(\mathrm{~s}, 12 \mathrm{H}), 1.38(\mathrm{t}, J=7.3 \mathrm{~Hz}, 3 \mathrm{H}), 3.69(\mathrm{dq}, J=6.3,7.3 \mathrm{~Hz}, 2 \mathrm{H}), 6.73$ (br t, $J=6.3 \mathrm{~Hz}, 1 \mathrm{H}), 7.03(\mathrm{~d}, J=7.8 \mathrm{~Hz}, 2 \mathrm{H}), 7.18(\mathrm{dd}, J=7.2,7.8 \mathrm{~Hz}, 2 \mathrm{H}), 7.23(\mathrm{~d}, J=7.2 \mathrm{~Hz}$, $1 \mathrm{H}), 7.42-7.51(\mathrm{~m}, 3 \mathrm{H}), 7.65(\mathrm{~d}, J=7.1 \mathrm{~Hz}, 1 \mathrm{H}), 7.80(\mathrm{~d}, J=8.0 \mathrm{~Hz}, 1 \mathrm{H}), 7.85(\mathrm{~d}, J=8.2 \mathrm{~Hz}$, $1 \mathrm{H}), 8.18(\mathrm{~d}, J=8.0 \mathrm{~Hz}, 1 \mathrm{H}) .{ }^{11} \mathbf{B}$ NMR (128 $\left.\mathbf{~ M H z}, \mathbf{C D C l}_{3}\right): \delta 15.3 .{ }^{13} \mathbf{C}$ NMR (100 $\left.\mathbf{~ M H z}, \mathbf{C D C l}_{3}\right): \delta 14.4,25.3,37.2$, 80.4, 80.9, 97.7, 116.9, 121.8, 124.8, 125.0, 125.1, 125.6, 127.9, 128.1, 128.1, 128.2, 128.9, 130.0, 131.4, 133.5, 136.9, 171.9. The carbon directly attached to the boron atom was not detected, likely due to quadrupolar relaxation. IR (ATR-IR): 2970, 2935, 2374, 2325, 1736, 1718, 1636, 1507, 1363, 1127, 973, 933, 761, 697, 586, $517 \mathrm{~cm}^{-1}$. HRMS (ESI): $m / z$ calculated for $\mathrm{C}_{29} \mathrm{H}_{31} \mathrm{BNO}_{3}{ }^{+}[\mathrm{M}+\mathrm{H}]^{+}=452.2392$, found: 452.2394 . mp: $93.1-94.0{ }^{\circ} \mathrm{C}$ (recrystallized from dichloromethane $/{ }^{\mathrm{n} h e x a n e)}$. Anal. (5a $\left.+\mathbf{H}_{2} \mathbf{O}\right)$ : Calcd: C, 74.21; H, 6.87; N, 2.98. Found: C, 74.39; H, 6.95; N, 3.06.

(E)-N-ethyl-2-(naphthalen-2-yl(4,4,5,5-tetramethyl-1,3,2-dioxaborolan-2-yl)methylene)-4-phenylbut-3-ynamid e $(5 \mathbf{b})$

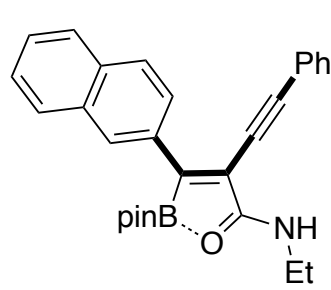

Following the General Procedure (MPLC: gradient, dichloromethane $/$ EtOAc $=100 / 0 \rightarrow$ $73 / 27$ ), the titled compound was obtained as a yellow solid in $87 \%$ yield ( $98.6 \mathrm{mg}$ ). The compound decomposed during recrystallization from dichloromethane $/{ }^{\mathrm{n}}$ hexane. ${ }^{\mathrm{l}} \mathbf{H}$ NMR $\left(400 \mathbf{~ M H z} \mathbf{C D C l}_{3}\right): \delta 1.32(\mathrm{~s}, 12 \mathrm{H}), 1.36(\mathrm{t}, J=7.3 \mathrm{~Hz}, 3 \mathrm{H}), 3.66(\mathrm{dq}, J=6.5,7.3 \mathrm{~Hz}, 2 \mathrm{H})$, $6.73(\mathrm{brt}, J=6.5 \mathrm{~Hz}, 1 \mathrm{H}), 7.34-7.36(\mathrm{~m}, 3 \mathrm{H}), 7.44-7.51(\mathrm{~m}, 4 \mathrm{H}), 7.82-7.89(\mathrm{~m}, 3 \mathrm{H}), 8.13(\mathrm{~d}, J$ $=8.4 \mathrm{~Hz}, 1 \mathrm{H}), 8.53(\mathrm{~s}, 1 \mathrm{H}) .{ }^{11} \mathbf{B}$ NMR $\left(128 \mathbf{M H z} \mathbf{C D C l}_{3}\right): \delta 15.2 .{ }^{13} \mathbf{C}$ NMR $(\mathbf{1 0 0} \mathbf{~ M H z}$, $\left.\mathbf{C D C l}_{3}\right): \delta 14.4,26.1,37.1,81.0,81.2,96.8,113.7,122.1,126.0,126.3,126.7,127.2,127.7$, $128.5,128.8,129.1,129.2,131.6,133.0,133.8,135.2,172.2$. The carbon directly attached to the boron atom was not detected, likely due to quadrupolar relaxation. IR (ATR-IR): 2986, 2970, 2372, 2326, 1737, 1647, 1634, 1525, 1363, 1227, 1117, 1044, 985, 863, 830, 750, 691, 659, 634, $538 \mathrm{~cm}^{-1}$. HRMS (ESI): $m / z$ calculated for $\mathrm{C}_{29} \mathrm{H}_{31} \mathrm{BNO}_{3}{ }^{+}[\mathrm{M}+\mathrm{H}]^{+}=452.2392$, found: 452.2394. Anal. $(5 \mathbf{b}+4 / 5$ chloroform): Calcd: $\mathrm{C}, 64.45 ; \mathrm{H}, 5.68 ; \mathrm{N}$, 2.56. Found: $\mathrm{C}, 64.14 ; \mathrm{H}, 5.63 ; \mathrm{N}, 2.47$. Elemental analysis was conducted after purification using the recycling preparative HPLC.

(E)-2-(benzo $[d][1,3]$ dioxol-5-yl (4,4,5,5-tetramethyl-1,3,2-dioxaborolan-2-yl)methylene)- $N$-ethyl-4-phenylbut3-ynamide (5c)

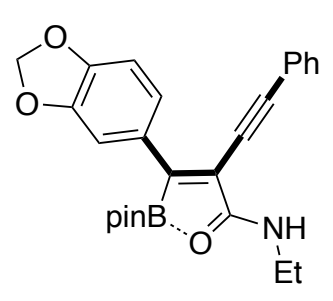

Following the General Procedure (MPLC: dichloromethane/EtOAc $=10 / 1$ ), the titled compound was obtained as a yellow solid in $85 \%$ yield $(94.9 \mathrm{mg}) .{ }^{1} \mathbf{H}$ NMR (400 MHz, $\left.\mathbf{C D C l}_{3}\right): \delta 1.30(\mathrm{~s}, 12 \mathrm{H}), 1.33(\mathrm{t}, J=7.3 \mathrm{~Hz}, 3 \mathrm{H}), 3.61(\mathrm{dq}, J=6.2,7.3 \mathrm{~Hz}, 2 \mathrm{H}), 5.99(\mathrm{~s}, 2 \mathrm{H})$, $6.64(\mathrm{brt}, J=6.2 \mathrm{~Hz}, 1 \mathrm{H}), 6.85(\mathrm{~d}, J=8.1 \mathrm{~Hz}, 1 \mathrm{H}), 7.35-7.37(\mathrm{~m}, 3 \mathrm{H}), 7.45-7.47(\mathrm{~m}, 2 \mathrm{H}), 7.64$ $(\mathrm{m}, 1 \mathrm{H}), 7.66(\mathrm{dd}, J=1.5,8.1 \mathrm{~Hz}, 1 \mathrm{H}) .{ }^{11} \mathbf{B}$ NMR $\left(128 \mathbf{M H z}, \mathbf{C D C l}_{3}\right): \delta 14.8 .{ }^{13} \mathbf{C}$ NMR $(\mathbf{1 0 0}$

MHz, $\left.\mathbf{C D C l}_{3}\right): \delta 14.4,26.2,37.0,80.9,81.4,97.1,101.1,107.9,109.2,111.7,122.2,124.6,128.5$, $129.0,131.6,131.7,147.2,148.8,172.4$. The carbon directly attached to the boron atom was not detected, likely due to quadrupolar relaxation. IR (ATR-IR): 2971, 2372, 2325, 1735, 1635, 1481, 1445, 1362, 1127, 1122, 1101, 1038, 976, 
880, 754, 690, 616, $546 \mathrm{~cm}^{-1}$. HRMS (ESI): $m / z$ calculated for $\mathrm{C}_{26} \mathrm{H}_{29} \mathrm{BNO}_{5}{ }^{+}[\mathrm{M}+\mathrm{H}]^{+}=446.2133$, found: 446.2133 . mp: $123.1-125.3^{\circ} \mathrm{C}$ (recrystallized from dichloromethane/" ${ }^{n}$ hexane). Anal. (5c + 1/5 H2 $\mathbf{O}$ ): Calcd: C, 69.56; H, 6.38; N, 3.12. Found: C, $69.61 ; \mathrm{H}, 6.55 ; \mathrm{N}, 2.97$.

(E)-N-ethyl-2-((4-methoxyphenyl)(4,4,5,5-tetramethyl-1,3,2-dioxaborolan-2-yl)methylene)-4-phenylbut-3-yna mide (5d)

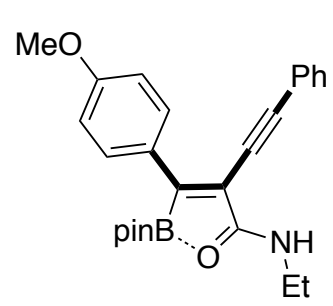

Following the General Procedure, the titled compound was obtained as a yellow solid in $65 \%$ yield $(70.0 \mathrm{mg})$ after purification using the recycling preparative HPLC. ${ }^{1} \mathbf{H}$ NMR $(400 \mathbf{~ M H z}$, $\left.\mathbf{C D C l}_{3}\right): \delta 1.31(\mathrm{~s}, 12 \mathrm{H}), 1.33(\mathrm{t}, J=7.3 \mathrm{~Hz}, 3 \mathrm{H}), 3.61(\mathrm{dq}, J=6.0,7.3 \mathrm{~Hz}, 2 \mathrm{H}), 3.84(\mathrm{~s}, 3 \mathrm{H})$, $6.64(\mathrm{br} \mathrm{t}, J=6.0 \mathrm{~Hz}, 1 \mathrm{H}), 6.92(\mathrm{~d}, J=9.0 \mathrm{~Hz}, 2 \mathrm{H}), 7.34-7.36(\mathrm{~m}, 3 \mathrm{H}), 7.45-7.47(\mathrm{~m}, 2 \mathrm{H}), 8.07$ $(\mathrm{d}, J=9.0 \mathrm{~Hz}, 2 \mathrm{H}) .{ }^{11} \mathbf{B}$ NMR (128 $\left.\mathbf{~ M H z}, \mathbf{C D C l}_{3}\right): \delta 15.3 .{ }^{13} \mathbf{C}$ NMR (100 $\left.\mathbf{M H z}, \mathbf{C D C l}_{3}\right): \delta$ $14.4,26.2,37.0,55.2,80.8,81.6,96.6,111.3,113.3,122.3,128.5,128.9,130.3,131.1,131.6$, $160.8,172.5$. The carbon directly attached to the boron atom was not detected, likely due to quadrupolar relaxation. IR (ATR-IR): 2970, 2369, 2328, 1736, 1718, 1635, 1507, 1364, 1217, 1127, 1026, 975, 754, 688, 558, 527, $506 \mathrm{~cm}^{-1}$. HRMS (ESI): $m / z$ calculated for $\mathrm{C}_{26} \mathrm{H}_{31} \mathrm{BNO}_{4}{ }^{+}[\mathrm{M}+\mathrm{H}]^{+}=432.2341$, found: 432.2343 . mp: $90.6-92.4{ }^{\circ} \mathrm{C}$ (recrystallized from dichloromethane $\left./{ }^{\mathrm{n} h e x a n e}\right)$. Anal. $\left(\mathbf{5 d}+\mathbf{H}_{2} \mathbf{O}\right)$ : Calcd: C, 69.50; H, 7.18; N, 3.12. Found: C, 69.23; H, 7.02; N, 3.08 .

(E)-N-ethyl-2-((4-(methylthio)phenyl)(4,4,5,5-tetramethyl-1,3,2-dioxaborolan-2-yl)methylene)-4-phenylbut-3ynamide (5e)

MeS

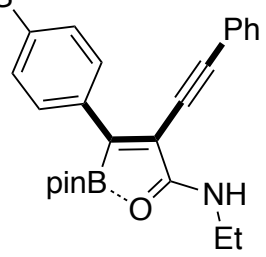

Following the General Procedure (MPLC: gradient, dichloromethane/EtOAc $=100 / 0 \rightarrow$ 88/12), the titled compound was obtained as a pale yellow solid in $93 \%$ yield $(103.5 \mathrm{mg}) .{ }^{1} \mathbf{H}$ NMR (400 MHz, $\left.\mathbf{C D C l}_{3}\right): \delta 1.29(\mathrm{~s}, 12 \mathrm{H}), 1.33(\mathrm{t}, J=7.2 \mathrm{~Hz}, 3 \mathrm{H}), 2.51(\mathrm{~s}, 3 \mathrm{H}), 3.62(\mathrm{dq}, J=$ 6.0, 7.2 Hz, 2H), 6.67 (br t, $J=6.0 \mathrm{~Hz}, 1 \mathrm{H}), 7.24-7.26(\mathrm{~m}, 2 \mathrm{H}), 7.35-7.39(\mathrm{~m}, 3 \mathrm{H}), 7.43-7.46$ $(\mathrm{m}, 2 \mathrm{H}), 7.97(\mathrm{~d}, J=8.4 \mathrm{~Hz}, 2 \mathrm{H}) .{ }^{11} \mathbf{B}$ NMR (128 $\left.\mathbf{M H z}, \mathbf{C D C l}_{3}\right): \delta 15.3 .{ }^{13} \mathbf{C}$ NMR (100 MHz, $\left.\mathbf{C D C l}_{3}\right): \delta 14.4,15.2,26.1,37.0,80.9,81.2,97.0,112.6,122.1,125.1,128.5,129.1,129.5,131.6$,

134.2, 140.5, 172.2. The carbon directly attached to the boron atom was not detected, likely due to quadrupolar relaxation. IR (ATR-IR): 2971, 2370, 2326, 1736, 1716, 1636, 1507, 1363, 1321, 1127, 1092, 973, 934, 752, 688, 633, 527, $507 \mathrm{~cm}^{-1}$. HRMS (ESI): $\mathrm{m} / z$ calculated for $\mathrm{C}_{26} \mathrm{H}_{31} \mathrm{BNO}_{3} \mathrm{~S}^{+}[\mathrm{M}+\mathrm{H}]^{+}=448.2112$, found: 448.2111. $\mathbf{~ m p : ~} 100.6{ }^{\circ} \mathrm{C}$ (recrystallized from dichloromethane $/{ }^{n}$ hexane). Anal. $\left(5 e+4 / 5 \mathbf{H}_{2} \mathbf{O}\right)$ : Calcd: C, 67.62; H, 6.90; N, 3.03. Found: C, $67.45 ; \mathrm{H}, 6.92 ; \mathrm{N}, 2.81$.

\section{(E)-2-((4-(diphenylamino)phenyl)(4,4,5,5-tetramethyl-1,3,2-dioxaborolan-2-yl)methylene)- $N$-ethyl-4-phenylb} ut-3-ynamide (5f)

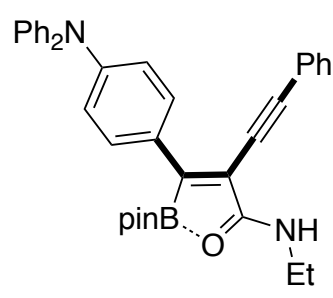

Following the General Procedure, the titled compound was obtained as a yellow solid in $77 \%$ yield (109.4 mg) after purification using the recycling preparative HPLC. ${ }^{1} \mathbf{H}$ NMR (400 MHz, $\left.\mathbf{C D C l}_{3}\right): \delta 1.31(\mathrm{~s}, 12 \mathrm{H}), 1.33(\mathrm{t}, J=7.4 \mathrm{~Hz}, 2 \mathrm{H}), 3.62(\mathrm{dq}, J=5.2,7.4 \mathrm{~Hz}, 2 \mathrm{H}), 6.62(\mathrm{br} \mathrm{d}, J=$ $5.2 \mathrm{~Hz}, 1 \mathrm{H}), 7.03(\mathrm{~d}, J=8.6 \mathrm{~Hz}, 2 \mathrm{H}), 7.06(\mathrm{dd}, J=7.2,7.9 \mathrm{~Hz}, 2 \mathrm{H}), 7.15(\mathrm{~d}, J=8.1 \mathrm{~Hz}, 4 \mathrm{H})$, $7.29(\mathrm{~d}, J=8.1 \mathrm{~Hz}, 4 \mathrm{H}), 7.33-7.35(\mathrm{~m}, 3 \mathrm{H}), 7.43-7.45(\mathrm{~m}, 2 \mathrm{H}), 8.04(\mathrm{~d}, J=8.6 \mathrm{~Hz}, 2 \mathrm{H}) .{ }^{11} \mathbf{B}$ NMR (128 MHz, $\left.\mathbf{C D C l}_{3}\right): \delta 14.7 .{ }^{13} \mathbf{C}$ NMR (100 MHz, $\left.\mathbf{C D C l}_{3}\right): \delta 14.3,26.2,36.9,80.7,82.0$, S-20 
97.1, 110.4, 120.8, 122.2, 123.5, 125.3, 128.4, 128.8, 129.3, 130.6, 130.7, 131.4, 147.1, 149.1, 172.4. The carbon directly attached to the boron atom was not detected, likely due to quadrupolar relaxation. IR (ATR-IR): 3391, 3063, 3037, 2970, 2934, 2225, 1630, 1587, 1484, 1327, 1279, 1180, 1126, 1048, 972, 910, 840, 753, 729, 694, 648, 615, 526, 510, 401 cm ${ }^{-1}$. HRMS (ESI): $m / z$ calculated for $\mathrm{C}_{37} \mathrm{H}_{37} \mathrm{BN}_{2} \mathrm{NaO}_{3}{ }^{+}[\mathrm{M}+\mathrm{Na}]^{+}=591.2789$, found: 591.2785. $\mathbf{m p :} 99.4-102.2$ ' $\mathrm{C}$ (recrystallized from dichloromethane/ ${ }^{\mathrm{n}}$ hexane). Anal. (5f $\left.+\mathbf{H}_{2} \mathbf{O}\right)$ : Calcd: C, 75.77; H, 6.70; N, 4.78. Found: C, 75.66; $\mathrm{H}, 6.96 ; \mathrm{N}, 4.70$.

(E)-2-((4-(9H-carbazol-9-yl)phenyl)(4,4,5,5-tetramethyl-1,3,2-dioxaborolan-2-yl)methylene)-N-ethyl-4-phenyl but-3-ynamide $(5 g)$

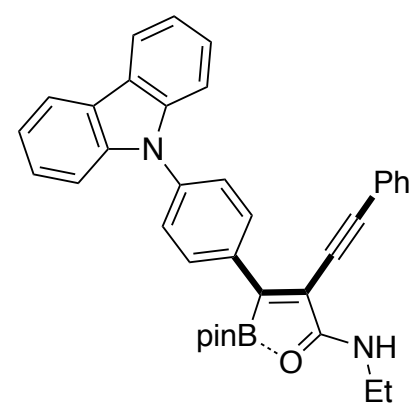

Following the General Procedure, the titled compound was obtained as an orange solid in $57 \%$ yield $\left(80.7 \mathrm{mg}\right.$ ) after purification using the recycling preparative HPLC. ${ }^{1} \mathbf{H}$ NMR (400 MHz, $\left.\mathbf{C D C l}_{3}\right): \delta 1.35(\mathrm{~s}, 12 \mathrm{H}), 1.38(\mathrm{t}, J=7.4 \mathrm{~Hz}, 3 \mathrm{H}), 3.67(\mathrm{dq}, J=6.2,7.4 \mathrm{~Hz}$, $2 \mathrm{H}), 6.74(\mathrm{br} t, J=6.2 \mathrm{~Hz}, 1 \mathrm{H}), 7.29(\mathrm{dd}, J=7.2,7.7 \mathrm{~Hz}, 2 \mathrm{H}), 7.30-7.36(\mathrm{~m}, 3 \mathrm{H})$, 7.39-7.44 (m, 2H), 7.47-7.52 (m, 4H), $7.63(\mathrm{~d}, J=8.4 \mathrm{~Hz}, 2 \mathrm{H}), 8.14(\mathrm{~d}, J=7.8 \mathrm{~Hz}, 2 \mathrm{H})$, $8.26(\mathrm{~d}, J=8.4 \mathrm{~Hz}, 2 \mathrm{H}) .{ }^{11} \mathbf{B}$ NMR (128 $\left.\mathbf{M H z}, \mathbf{C D C l}_{3}\right): \delta 15.2 .{ }^{13} \mathrm{C}$ NMR (100 MHz, $\left.\mathbf{C D C l}_{3}\right): \delta 14.4,26.1,37.2,81.0,81.1,97.4,110.0,113.8,120.1,120.3,121.9,123.5,126.0$, $126.0,128.6,129.3,130.6,131.7,136.4,138.5,140.6,172.1$. The carbon directly attached to the boron atom was not detected, likely due to quadrupolar relaxation. IR (ATR-IR): 3734, 3629, 2992, 2970, 2925, 2372, 2327, 1735, 1653, 1600, 1507, 1449, 1362, 1228, 1174, 1129, 1041, 975, 935, 863, 748, 723, 689, 662, 618, 531, $507 \mathrm{~cm}^{-1}$. HRMS (ESI): $\mathrm{m} / z$ calculated for $\mathrm{C}_{37} \mathrm{H}_{36} \mathrm{BN}_{2} \mathrm{O}_{3}{ }^{+}[\mathrm{M}+\mathrm{H}]^{+}=567.2813$, found: 567.2813 . mp: $107.0-108.0{ }^{\circ} \mathrm{C}$ (recrystallized from dichloromethane $/{ }^{n}$ hexane). Anal. $\left(\mathbf{5 g}+\mathbf{H}_{2} \mathbf{O}+\mathbf{1} / \mathbf{6}^{\mathrm{n}} \mathbf{h e x a n e}^{\mathrm{a}}\right.$ : Calcd: C, 76.38; H, 6.85; N, 4.57. Found: C, 76.09; H, 6.82; N, 4.60 .

(E)-2-((4-chlorophenyl) (4,4,5,5-tetramethyl-1,3,2-dioxaborolan-2-yl)methylene)- $N$-ethyl-4-phenylbut-3-ynami de $(5 h)$

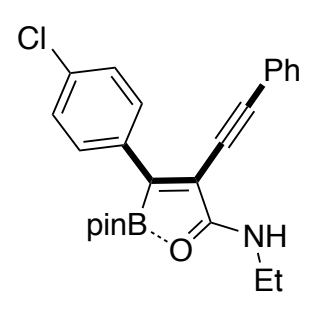

Following the General Procedure (MPLC: gradient, dichloromethane $/$ EtOAc $=100 / 0 \rightarrow 71 / 29$ ), the titled compound was obtained as a yellow solid in $79 \%$ yield $(85.5 \mathrm{mg}) .{ }^{1} \mathbf{H}$ NMR (400 MHz, $\left.\mathbf{C D C l}_{3}\right): \delta 1.28(\mathrm{~s}, 12 \mathrm{H}), 1.34(\mathrm{t}, J=7.3 \mathrm{~Hz}, 3 \mathrm{H}), 3.63(\mathrm{dq}, J=6.2,7.3 \mathrm{~Hz}, 2 \mathrm{H}), 6.69(\mathrm{br} \mathrm{t}, J=6.2$ $\mathrm{Hz}, 1 \mathrm{H}), 7.34-7.38(\mathrm{~m}, 3 \mathrm{H}), 7.37(\mathrm{~d}, J=8.5 \mathrm{~Hz}, 2 \mathrm{H}), 7.42-7.45(\mathrm{~m}, 2 \mathrm{H}), 7.91(\mathrm{~d}, J=8.5 \mathrm{~Hz}, 2 \mathrm{H})$.

${ }^{11}$ B NMR (128 MHz, $\left.\mathbf{C D C l}_{3}\right): \delta 15.0 .{ }^{13} \mathbf{C} \mathbf{~ N M R}\left(100 \mathbf{~ M H z}, \mathbf{C D C l}_{3}\right): \delta 14.3,25.9,37.1,80.6,81.0$, $97.1,113.9,121.8,128.1,128.6,129.2,130.2,131.7,135.1,136.0,172.0$. The carbon directly attached to the boron atom was not detected, likely due to quadrupolar relaxation. IR (ATR-IR): 2971, 2928, 2375, 2309, 1735, 1645, 1507, 1449, 1363, 1318, 1228, 1174, 1120, 1015, 972, 933, 895, 834, 805, 751, 721, 688, 618, $531 \mathrm{~cm}^{-1}$. HRMS (ESI): $m / z$ calculated for $\mathrm{C}_{25} \mathrm{H}_{28} \mathrm{BClNO}_{3}{ }^{+}[\mathrm{M}+\mathrm{H}]^{+}=436.1845$, found: 436.1845. mp: $79.9-81.6{ }^{\circ} \mathrm{C}$ (recrystallized from dichloromethane $/{ }^{\mathrm{n} h e x a n e)}$. Anal. $\left(\mathbf{5 h}+\mathbf{H}_{2} \mathbf{O}\right)$ : Calcd: C, 66.17; H, 6.44; N, 3.09. Found: C, 66.32; $\mathrm{H}, 6.64$; N, 3.02. 
(E)-N-ethyl-4-phenyl-2-((4,4,5,5-tetramethyl-1,3,2-dioxaborolan-2-yl)(4-(trifluoromethyl)phenyl)methylene)b ut-3-ynamide (5i)

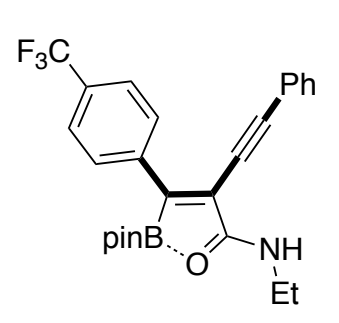

Following the General Procedure, the titled compound was obtained as a pale yellow solid in $82 \%$ yield $(96.7 \mathrm{mg})$ after purification using the recycling preparative HPLC. ${ }^{1} \mathbf{H}$ NMR (400 MHz, $\left.\mathbf{C D C l}_{3}\right): \delta 1.27(\mathrm{~s}, 12 \mathrm{H}), 1.36(\mathrm{t}, J=7.3 \mathrm{~Hz}, 3 \mathrm{H}), 3.65(\mathrm{dq}, J=6.6,7.3 \mathrm{~Hz}, 2 \mathrm{H}), 6.75(\mathrm{brt}$, $J=6.6 \mathrm{~Hz}, 1 \mathrm{H}), 7.36-7.44(\mathrm{~m}, 5 \mathrm{H}), 7.65(\mathrm{~d}, J=8.1 \mathrm{~Hz}, 2 \mathrm{H}), 8.01(\mathrm{~d}, J=8.1 \mathrm{~Hz}, 2 \mathrm{H}) .{ }^{11} \mathbf{B} \mathbf{N M R}$ (128 MHz, $\left.\mathbf{C D C l}_{3}\right): \delta 15.3 .{ }^{13} \mathbf{C}$ NMR (100 $\left.\mathbf{~ M H z}, \mathbf{C D C l}_{3}\right): \delta 14.3,25.9,37.2,80.1,81.0,97.3$, $115.3,121.6,124.2(q, J=270.5 \mathrm{~Hz}), 124.7(\mathrm{q}, J=3.8 \mathrm{~Hz}), 128.6,128.7,129.4,130.5$ (q, $J=32.1$ $\mathrm{Hz}), 131.7,141.1,171.7$. The carbon directly attached to the boron atom was not detected, likely due to quadrupolar relaxation. ${ }^{19}$ F NMR (376 $\mathbf{~ M H z}, \mathbf{C D C l}_{3}$ ): $\delta$-62.53. IR (ATR-IR): 2971, 2371, 2325, 1736, 1717, 1646, 1507, 1365, 1321, 1166, 1019, 973, 935, 855, 754, 688, 584, $527 \mathrm{~cm}^{-1}$. HRMS (ESI): $m / z$ calculated for $\mathrm{C}_{26} \mathrm{H}_{28} \mathrm{BF}_{3} \mathrm{NO}_{3}^{+}[\mathrm{M}+\mathrm{H}]^{+}=$ 470.2109, found: 470.2109. mp: 143.0-143.3 ${ }^{\circ} \mathrm{C}$ (recrystallized from dichloromethane $/{ }^{\mathrm{n} h e x a n e)}$. Anal. $(5 \mathbf{i}+$ $\mathbf{H}_{2} \mathbf{O}$ ): Calcd: C, 64.08; H, 6.00; N, 2.87. Found: C, 64.16; H, 5.96; N, 2.87.

Ethyl (E)-2-(2-(ethylcarbamoyl)-4-phenyl-1-(4,4,5,5-tetramethyl-1,3,2-dioxaborolan-2-yl)but-1en-3-yn-1-yl) benzoate $(5 j)$

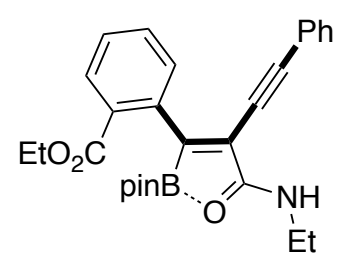

Following the General Procedure, the titled compound was obtained as a yellow solid in 67\% yield $(79.3 \mathrm{mg})$ after purification using the recycling preparative HPLC. ${ }^{1} \mathbf{H}$ NMR (400 MHz, $\left.\mathbf{C D C l}_{3}\right): \delta 1.09(\mathrm{~s}, 12 \mathrm{H}), 1.26(\mathrm{t}, J=7.1 \mathrm{~Hz}, 3 \mathrm{H}), 1.35(\mathrm{t}, J=7.3 \mathrm{~Hz}, 3 \mathrm{H}), 3.65(\mathrm{dq}, J=6.8,7.1$ $\mathrm{Hz}, 2 \mathrm{H}), 4.27(\mathrm{q}, J=7.3 \mathrm{~Hz}, 2 \mathrm{H}), 6.67(\mathrm{br} \mathrm{t}, J=6.8 \mathrm{~Hz}, 1 \mathrm{H}), 7.25-7.35(\mathrm{~m}, 6 \mathrm{H}), 7.46-7.52(\mathrm{~m}$, 2H), $7.90(\mathrm{dd}, J=0.60,8.2 \mathrm{~Hz}, 1 \mathrm{H}) .{ }^{11} \mathbf{B}$ NMR $\left(128 \mathbf{M H z} \mathbf{C D C l}_{3}\right): \delta 15.3 .{ }^{13} \mathbf{C}$ NMR $(100$

MHz, $\left.\mathbf{C D C l}_{3}\right): \delta 14.1,14.3,25.1,37.1,61.1,79.8,80.6,96.2,114.6,122.0,127.1,127.5,128.3,128.9,129.6,129.7,131.0$, $131.7,139.8,167.4,171.9$. The carbon directly attached to the boron atom was not detected, likely due to quadrupolar relaxation. IR (ATR-IR): 3534, 3279, 2970, 2374, 2327, 1711, 1652, 1522, 1489, 1361, 1261, 1174, 1117, 1034, 972, 935, 876, 760, 723, 690, 632, 580, $519 \mathrm{~cm}^{-1}$. HRMS (ESI): $\mathrm{m} / z$ calculated for $\mathrm{C}_{28} \mathrm{H}_{33} \mathrm{BNO}_{5}{ }^{+}[\mathrm{M}+\mathrm{H}]^{+}=474.2446$, found: 474.2446 . mp: $82.3-83.7^{\circ} \mathrm{C}$ (recrystallized from dichloromethane $/{ }^{\mathrm{n}}$ hexane). Anal. (5j $+\mathbf{2} \mathbf{H}_{2} \mathbf{O}$ ): Calcd: C, 66.02; H, 7.12; N, 2.75. Found: C, 65.95; H, 7.01; N, 2.69.

(E)-2-((4-cyanophenyl) (4,4,5,5-tetramethyl-1,3,2-dioxaborolan-2-yl)methylene)- $N$-ethyl-4-phenylbut-3-ynami de $(5 k)$

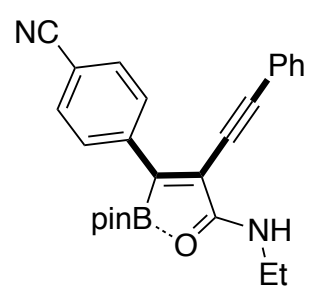

Following the General Procedure (MPLC: gradient, dichloromethane $/$ EtOAc $=100 / 0 \rightarrow$ $73 / 27)$, the titled compound was obtained as an orange solid in $57 \%$ yield $(45.1 \mathrm{mg}) .{ }^{1} \mathbf{H}$ NMR (400 MHz, $\left.\mathbf{C D C l}_{3}\right): \delta 1.26(\mathrm{~s}, 12 \mathrm{H}), 1.36(\mathrm{t}, J=7.3 \mathrm{~Hz}, 3 \mathrm{H}), 3.66(\mathrm{dq}, J=6.6,7.3 \mathrm{~Hz}, 2 \mathrm{H}), 6.76$ $($ br t, $J=6.6 \mathrm{~Hz}, 1 \mathrm{H}), 7.36-7.43(\mathrm{~m}, 5 \mathrm{H}), 7.68(\mathrm{~d}, J=8.1 \mathrm{~Hz}, 2 \mathrm{H}), 7.97(\mathrm{~d}, J=8.1 \mathrm{~Hz}, 2 \mathrm{H}) .{ }^{11} \mathbf{B}$ NMR (128 MHz, $\left.\mathbf{C D C l}_{3}\right): \delta 14.2 .{ }^{13} \mathbf{C}$ NMR (100 $\left.\mathbf{~ M H z , ~} \mathbf{C D C l}_{3}\right): \delta 14.3,25.8,37.3,79.7,81.0$, 97.7, 112.0, 115.9, 119.1, 121.3, 128.6, 128.9, 129.6, 131.6, 131.7, 142.3, 171.5. The carbon directly attached to the boron atom was not detected, likely due to quadrupolar relaxation. IR (ATR-IR): 3734, 3649, 2970, 2370, 2315, 2226, 1734, 1646, 1507, 1363, 1123, 973, 860, 752, 633, 552, $517 \mathrm{~cm}^{-1}$. HRMS (ESI): $\mathrm{m} / z$ calculated for $\mathrm{C}_{26} \mathrm{H}_{28} \mathrm{BN}_{2} \mathrm{O}_{3}{ }^{+}[\mathrm{M}+\mathrm{H}]^{+}=427.2187$, found: 427.2187. mp: 154.8-155.8 ${ }^{\circ} \mathrm{C}$ (recrystallized from dichloromethane/"nhexane). Anal. (5k+3/5 $\left.\mathbf{H}_{2} \mathbf{O}\right)$ : Calcd: C, 71.44; H, 6.50; N, 6.41. Found: C, 71.47; H, 6.54; N, 6.36. 
(E)-N-ethyl-2-((4-nitrophenyl)(4,4,5,5-tetramethyl-1,3,2-dioxaborolan-2-yl)methylene)-4-phenylbut-3-ynamid e (51)

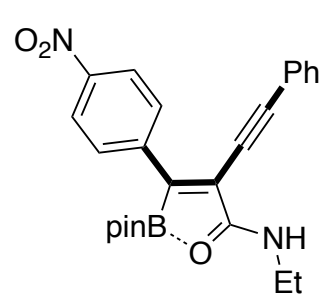

Following the General Procedure (MPLC: dichloromethane/EtOAc $=10 / 1$ ), the titled compound was obtained as an orange solid in $30 \%$ yield $(33.2 \mathrm{mg}) .{ }^{1} \mathbf{H}$ NMR (400 MHz, $\left.\mathbf{C D C l}_{3}\right): \delta 1.26(\mathrm{~s}, 12 \mathrm{H}), 1.37(\mathrm{t}, J=7.3 \mathrm{~Hz}, 3 \mathrm{H}), 3.66(\mathrm{dq}, J=6.2,7.3 \mathrm{~Hz}, 2 \mathrm{H}), 6.76(\mathrm{br} \mathrm{t}, J=$ $6.2 \mathrm{~Hz}, 1 \mathrm{H}), 7.36-7.43(\mathrm{~m}, 5 \mathrm{H}), 8.02(\mathrm{~d}, J=8.9 \mathrm{~Hz}, 2 \mathrm{H}), 8.25(\mathrm{~d}, J=8.9 \mathrm{~Hz}, 2 \mathrm{H}) .{ }^{11} \mathbf{B}$ NMR (128 MHz, $\left.\mathbf{C D C l}_{3}\right): \delta 14.4 .{ }^{13} \mathbf{C}$ NMR (100 $\left.\mathbf{~ M H z , ~} \mathbf{C D C l}_{3}\right): \delta$ 14.2, 25.8, 37.4, 79.6, 81.1, 97.9, $116.3,121.3,123.1,128.7,129.1,129.6,131.8,144.4,147.7,171.5$. The carbon directly attached to the boron atom was not detected, likely due to quadrupolar relaxation. IR (ATR-IR): 3734, 3649, 2970, 2373, 2317, 1735, 1647, 1508, 1341, 1315, 1277, 1122, 973, 935, 853, 752, 687, 633, 518, $506 \mathrm{~cm}^{-1}$. HRMS (ESI): $\mathrm{m} / z$ calculated for $\mathrm{C}_{25} \mathrm{H}_{28} \mathrm{BN}_{2} \mathrm{O}_{5}^{+}[\mathrm{M}+\mathrm{H}]^{+}=447.2086$, found: 447.2086 . mp: $170.7^{\circ} \mathrm{C}$ (recrystallized from dichloromethane $/{ }^{\mathrm{n} h e x a n e}$ ). Anal. (5I + $\mathbf{H}_{2} \mathbf{O}$ ): Calcd: C, 64.67; H, 6.30; N, 6.03. Found: C, 64.83; H, 6.48; N, 6.16.

(E)-N-ethyl-2-(mesityl(4,4,5,5-tetramethyl-1,3,2-dioxaborolan-2-yl)methylene)-4-phenylbut-3-ynamide (5m)

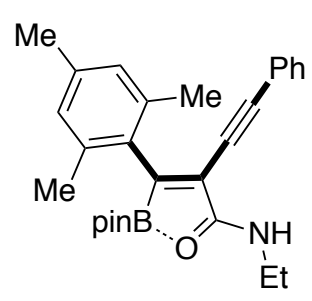

Following the General Procedure (MPLC: gradient, dichloromethane/EtOAc $=90 / 10 \rightarrow$ $39 / 61)$, the titled compound was obtained as a slightly brown solid in $28 \%$ yield $(30.5 \mathrm{mg}) .{ }^{1} \mathbf{H}$ NMR (400 MHz, CDCl 3 ): $\delta 1.06(\mathrm{~s}, 12 \mathrm{H}), 1.35(\mathrm{t}, J=7.4 \mathrm{~Hz}, 3 \mathrm{H}), 2.23(\mathrm{~s}, 6 \mathrm{H}), 2.26(\mathrm{~s}, 3 \mathrm{H})$, $3.65(\mathrm{dq}, J=5.7,7.4 \mathrm{~Hz}, 2 \mathrm{H}), 6.64(\mathrm{br} \mathrm{t}, J=5.7 \mathrm{~Hz}, 1 \mathrm{H}), 6.80(\mathrm{~s}, 2 \mathrm{H}), 7.27-7.30(\mathrm{~m}, 5 \mathrm{H}) .{ }^{11} \mathbf{B}$ NMR (128 MHz, $\left.\mathbf{C D C l}_{3}\right): \delta 15.1 .{ }^{13} \mathbf{C}$ NMR (100 $\left.\mathbf{M H z}, \mathbf{C D C l}_{3}\right): \delta 14.3,20.1,21.0,24.8,37.1$, $79.7,80.6,96.0,116.2,121.9,127.3,128.3,128.9,131.7,133.3,135.1,135.3,171.9$. The carbon directly attached to the boron atom was not detected, likely due to quadrupolar relaxation. IR (ATR-IR): 3396, 2972, 2921, 2223, 1634, 1528, 1443, 1363, 1307, 1279, 1181, 1160, 1117, 1037, 1002, 972, 911, 850, 755, 728, 690, 643, 534, $505 \mathrm{~cm}^{-1}$. HRMS (ESI): $m / z$ calculated for $\mathrm{C}_{28} \mathrm{H}_{34} \mathrm{BNNaO}_{3}{ }^{+}[\mathrm{M}+\mathrm{Na}]^{+}=466.2524$, found: 466.2513. mp: 149.1-150.5 ${ }^{\circ} \mathrm{C}$ (recrystallized from dichloromethane/ ${ }^{\mathrm{n} h e x a n e)}$. Anal. $\left(\mathbf{5 m}+\mathbf{1} / \mathbf{1 0} \mathbf{H}_{2} \mathbf{O}\right)$ : Calcd: C, 75.54; H, 7.74; N, 3.15. Found: C, 75.50; H, 7.64; N, 3.15 .

(E)-N-ethyl-4-phenyl-2-((4,4,5,5-tetramethyl-1,3,2-dioxaborolan-2-yl)(thiophen-3-yl)methylene)but-3-ynamid e (5n)

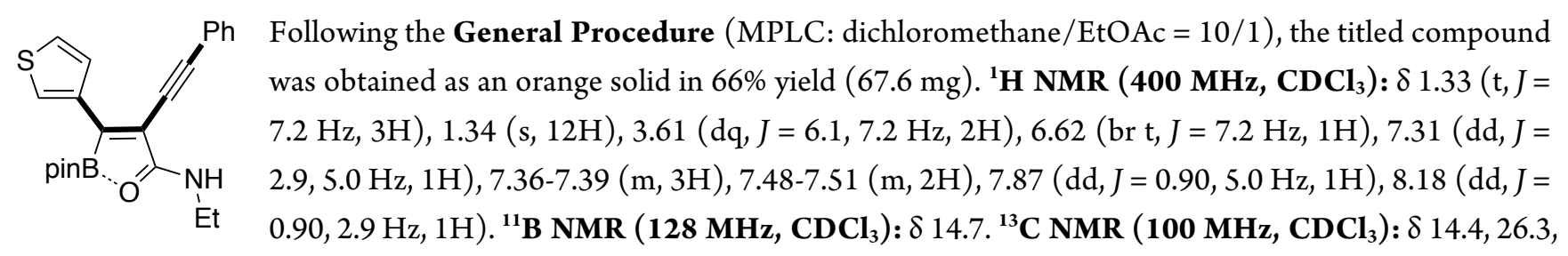
$37.0,81.0,81.7,97.7,111.4,122.2,124.7,128.0,128.6,129.1,129.6,131.6,138.9,172.4$. The carbon directly attached to the boron atom was not detected, likely due to quadrupolar relaxation. IR (ATR-IR): 2962, 2373, 2318, 1734, 1635, 1508, 1362, 1295, 1213, 1115, 1023, 977, 874, 788, 751, 688, 578, 520, $506 \mathrm{~cm}^{-1}$. HRMS (ESI): $m / z$ calculated for $\mathrm{C}_{23} \mathrm{H}_{27} \mathrm{BNO}_{3} \mathrm{~S}^{+}[\mathrm{M}+\mathrm{H}]^{+}=408.1799$, found: 408.1799. mp: 166.1-167.3 ${ }^{\circ} \mathrm{C}$ (decomp.; recrystallized from dichloromethane/"nhexane). Anal. (5n + 1/2 $\left.\mathbf{H}_{2} \mathbf{O}\right)$ : Calcd: C, 66.35; H, 6.54; N, 3.36. Found: C, 66.12; H, 6.30; N, 3.31. 
(E)-2-(benzo $[b]$ thiophen-2-yl (4,4,5,5-tetramethyl-1,3,2-dioxaborolan-2-yl)methylene)- $N$-ethyl-4-phenylbut-3-y namide (5o)

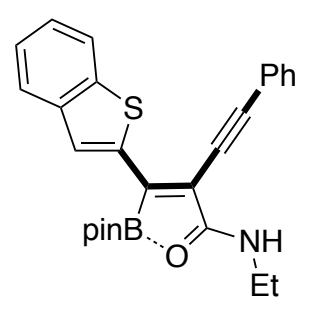

Following the General Procedure (MPLC: gradient, dichloromethane $/$ EtOAc $=100 / 0 \rightarrow 78 / 22$ ), the titled compound was obtained as yellow sticky oil in $55 \%$ yield $(63.1 \mathrm{mg})$. The compound decomposed during recrystallization from dichloromethane $/{ }^{\mathrm{n}}$ hexane. ${ }^{1} \mathbf{H}$ NMR (400 MHz, $\left.\mathbf{C D C l}_{3}\right): \delta 1.35(\mathrm{t}, J=7.4 \mathrm{~Hz}, 3 \mathrm{H}), 1.41(\mathrm{~s}, 12 \mathrm{H}), 3.64(\mathrm{dq}, J=6.8,7.4 \mathrm{~Hz}, 2 \mathrm{H}), 6.68(\mathrm{br} \mathrm{t}, J=6.8$ $\mathrm{Hz}, 1 \mathrm{H}), 7.34-7.36(\mathrm{~m}, 2 \mathrm{H}), 7.42-7.43(\mathrm{~m}, 3 \mathrm{H}), 7.62-7.63(\mathrm{~m}, 2 \mathrm{H}), 7.83-7.85(\mathrm{~m}, 2 \mathrm{H}), 8.02$ (s, 1H). ${ }^{11}$ B NMR (128 MHz, $\left.\mathbf{C D C l}_{3}\right): \delta 15.2 .{ }^{13} \mathbf{C}$ NMR (100 MHz, $\left.\mathbf{C D C l}_{3}\right): \delta$ 14.4, 26.6, 37.1, 81.1, $81.5,100.5,111.9,122.1,122.3,124.3,124.8,125.9,128.6,129.3,131.0,131.6,139.0,140.3,142.1,171.8$. The carbon directly attached to the boron atom was not detected, likely due to quadrupolar relaxation. IR (ATR-IR): 2978, 2931, 2369, 2336, 1634, 1508, 1328, 1117, 1043, 970, 849, 747, 725, 691, $546 \mathrm{~cm}^{-1}$. HRMS (ESI): $\mathrm{m} / z$ calculated for $\mathrm{C}_{27} \mathrm{H}_{29} \mathrm{BNO}_{3} \mathrm{~S}^{+}[\mathrm{M}+\mathrm{H}]^{+}=458.1956$, found: 458.1956. Anal. (5o + 3/5 $\left.\mathbf{H}_{2} \mathbf{O}\right)$ : Calcd: C, 69.26; H, 6.29; N, 2.99. Found: C, 69.23; H, 6.16; N, 3.01.

(E)-2-(dibenzo $[b, d]$ thiophen-2-yl (4,4,5,5-tetramethyl-1,3,2-dioxaborolan-2-yl)methylene)- $N$-ethyl-4-phenylbut -3-ynamide (5p)

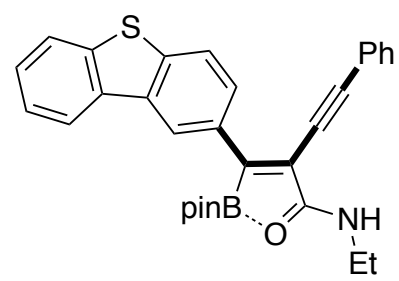

Following the General Procedure (MPLC: gradient, dichloromethane $/ \mathrm{EtOAc}=100 / 0 \rightarrow$ $78 / 22)$, the titled compound was obtained as yellow sticky oil in $84 \%$ yield (106.6 $\mathrm{mg}$ ). The compound decomposed during recrystallization from dichloromethane/ $/{ }^{\mathrm{n}}$ hexane. ${ }^{1} \mathbf{H}$ NMR (400 MHz, $\left.\mathbf{C D C l}_{3}\right): \delta 1.33(\mathrm{t}, J=7.2 \mathrm{~Hz}, 3 \mathrm{H}), 1.34(\mathrm{~s}, 12 \mathrm{H}), 3.61(\mathrm{dq}, J=6.1,7.2$ $\mathrm{Hz}, 2 \mathrm{H}), 6.62(\mathrm{br} \mathrm{t}, J=7.2 \mathrm{~Hz}, 1 \mathrm{H}), 7.31(\mathrm{dd}, J=2.9,5.0 \mathrm{~Hz}, 1 \mathrm{H}), 7.36-7.39(\mathrm{~m}, 2 \mathrm{H})$, 7.48-7.51 (m, 4H), $7.87(\mathrm{dd}, J=0.90,5.0 \mathrm{~Hz}, 2 \mathrm{H}), 8.18(\mathrm{dd}, J=0.90,2.9 \mathrm{~Hz}, 2 \mathrm{H}), 8.89(\mathrm{~s}$, 1H). ${ }^{11}$ B NMR (128 MHz, $\left.\mathbf{C D C l}_{3}\right): \delta 15.3 .{ }^{13} \mathbf{C}$ NMR (100 $\left.\mathbf{~ M H z , ~} \mathbf{C D C l}_{3}\right): \delta$ 14.4, 26.1, 37.1, 80.9, 81.2, 97.0, 113.1, $121.5,122.0,122.1,122.5,122.9,124.6,126.7,127.4,128.5,129.1,131.6,133.9,135.2,135.8,139.6,140.7,172.2$. The carbon directly attached to the boron atom was not detected, likely due to quadrupolar relaxation. IR (ATR-IR): 2972, $1633,1518,1463,1362,1228,1220,1180,1221,1079,1024,972,951,866,818,749,689,665,620,577,531 \mathrm{~cm}^{-1}$. HRMS (ESI): $m / z$ calculated for $\mathrm{C}_{31} \mathrm{H}_{31} \mathrm{BNO}_{3} \mathrm{~S}^{+}[\mathrm{M}+\mathrm{H}]^{+}=508.2112$, found: 508.2112. Anal. $(\mathbf{5 p}+7 / 6$ chloroform): Calcd: C, 59.74; H, 4.86; N, 2.17. Found: C, 59.94; H, 5.02; N, 2.17. Elemental analysis was conducted after purification using the recycling preparative HPLC.

(E)-2-(cyclohex-1-en-1-yl(4,4,5,5-tetramethyl-1,3,2-dioxaborolan-2-yl)methylene)- $N$-ethyl-4-phenylbut-3-ynam ide $(5 \mathbf{r})$

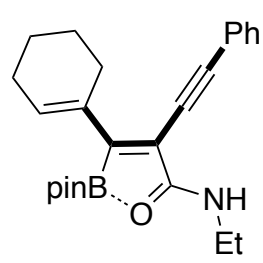

Following the General Procedure, the titled compound was obtained as a colorless solid in 27\% yield $(27.1 \mathrm{mg})$ after purification using the recycling preparative HPLC. ${ }^{1} \mathbf{H}$ NMR (400 MHz, $\left.\mathbf{C D C l}_{3}\right): \delta 0.96(\mathrm{~s}, 12 \mathrm{H}), 1.24(\mathrm{t}, J=7.2 \mathrm{~Hz}, 3 \mathrm{H}), 1.66-1.73(\mathrm{~m}, 4 \mathrm{H}), 2.32(\mathrm{t}, J=5.8 \mathrm{~Hz}, 2 \mathrm{H}), 2.81$ $(\mathrm{t}, J=5.9 \mathrm{~Hz}, 2 \mathrm{H}), 3.49(\mathrm{dq}, J=6.6,7.2 \mathrm{~Hz}, 2 \mathrm{H}), 6.19(\mathrm{br} \mathrm{s}, 1 \mathrm{H}), 7.18(\mathrm{~s}, 1 \mathrm{H}), 7.21-7.23(\mathrm{~m}, 2 \mathrm{H})$, 7.29-7.36 (m, 3H). ${ }^{11}$ B NMR (128 MHz, $\left.\mathbf{C D C l}_{3}\right): \delta 28.8 .{ }^{13} \mathbf{C}$ NMR (100 MHz, $\left.\mathbf{C D C l}_{3}\right): \delta 14.9$, $22.7,23.3,25.1,28.2,30.2,35.2,82.7,123.4,126.8,127.7,130.0,133.8,137.2,138.7,141.1,146.3,168.8$. The carbon directly attached to the boron atom was not detected, likely due to quadrupolar relaxation. IR (ATR-IR): 2927, 1636, 1538, 1440, 1379, 1334, 1216, 1152, 1105, 1017, 956, 884, 814, 779, 699, 671, $576 \mathrm{~cm}^{-1}$. HRMS (ESI): $m / z$ calculated 
for $\mathrm{C}_{25} \mathrm{H}_{33} \mathrm{BNO}_{3}{ }^{+}[\mathrm{M}+\mathrm{H}]^{+}=\quad 406.2548, \quad$ found: 406.2547. $\mathbf{m p :}$ 98.1-98.6 $\quad{ }^{\circ} \mathrm{C} \quad$ (recrystallized from dichloromethane/"nhexane). Anal. (5r + 6/5 $\mathbf{H}_{2} \mathbf{O}$ ): Calcd: C, 70.33; H, 8.12; N, 3.28. Found: C, 70.22; H, 7.84; N, 3.42.

(2E,4E)-N-ethyl-2-(phenylethynyl)-3-(4,4,5,5-tetramethyl-1,3,2-dioxaborolan-2-yl)-5-(4-(trifluoromethyl)phen yl)penta-2,4-dienamide (5s)

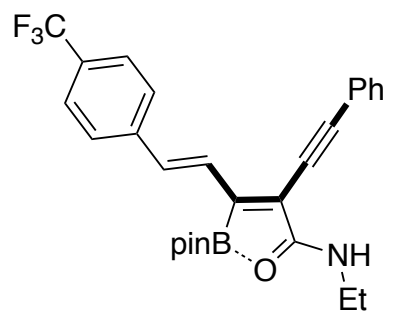

Following the General Procedure (MPLC: gradient, dichloromethane/EtOAc $=100 / 0 \rightarrow$ $71 / 29$ ) the titled compound was obtained as a yellow solid in $42 \%$ yield ( $51.8 \mathrm{mg}$ ). The compound decomposed during recrystallization from dichloromethane/ ${ }^{\mathrm{n} h e x a n e} .{ }^{1} \mathbf{H}$ NMR (400 MHz, $\left.\mathbf{C D C l}_{3}\right): \delta 1.33(\mathrm{t}, J=7.2 \mathrm{~Hz}, 3 \mathrm{H}), 1.39(\mathrm{~s}, 12 \mathrm{H}), 3.61(\mathrm{dq}, J=6.2,7.2 \mathrm{~Hz}, 2 \mathrm{H})$, 6.53 (br t, $J=6.2 \mathrm{~Hz}, 1 \mathrm{H}), 7.37-7.49(\mathrm{~m}, 4 \mathrm{H}), 7.50-7.56(\mathrm{~m}, 2 \mathrm{H}), 7.56-7.66(\mathrm{~m}, 5 \mathrm{H}) .{ }^{11} \mathbf{B}$

NMR (128 MHz, $\left.\mathbf{C D C l}_{3}\right): \delta 17.2 .{ }^{13} \mathbf{C}$ NMR (100 $\left.\mathbf{~ M H z}, \mathbf{C D C l}_{3}\right): \delta 14.4,26.3,36.9,80.0$, $81.2,99.9,116.3,121.9,124.0(\mathrm{q}, J=269.5 \mathrm{~Hz}), 125.6(\mathrm{q}, J=3.7 \mathrm{~Hz}), 127.5,128.5,128.6,129.3,130.2(\mathrm{q}, J=33.1 \mathrm{~Hz})$, $131.7,139.3,140.3,171.0$. The carbon directly attached to the boron atom was not detected, likely due to quadrupolar relaxation. ${ }^{19}$ F NMR (376 $\mathbf{~ M H z} \mathbf{C D C l}_{3}$ ): $\delta$-62.57. IR (ATR-IR): 2977, 2925, 1637, 1613, 1521, 1323, 1159, 1110, 1065, 1031, 963, 872, 829, 752, 689, $508 \mathrm{~cm}^{-1}$. HRMS (ESI): $m / z$ calculated for $\mathrm{C}_{28} \mathrm{H}_{30} \mathrm{BF}_{3} \mathrm{NO}_{3}{ }^{+}[\mathrm{M}+\mathrm{H}]^{+}=496.2265$, found: 496.2264. Anal.: Calcd: C, 67.89; H, 5.90; N, 2.83. Found: C, 67.84; H, 2.83; N, 5.91.

\section{(E)-N-ethyl-2-(phenylethynyl)-3-(4,4,5,5-tetramethyl-1,3,2-dioxaborolan-2-yl)but-2-enamide (5t)}

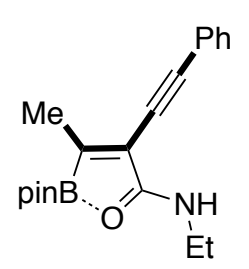

Following the General Procedure, the titled compound was obtained as a colorless solid in $10 \%$ yield (8.6 mg) after purification using the recycling preparative HPLC. ${ }^{1} \mathbf{H}$ NMR (400 $\left.\mathbf{M H z}, \mathbf{C D C l}_{3}\right): \delta 1.27$ $(\mathrm{s}, 12 \mathrm{H}), 1.30(\mathrm{t}, J=7.4 \mathrm{~Hz}, 3 \mathrm{H}), 2.19(\mathrm{~s}, 3 \mathrm{H}), 3.59(\mathrm{dq}, J=6.7,7.4 \mathrm{~Hz}, 2 \mathrm{H}), 6.45(\mathrm{br} \mathrm{t}, J=6.7 \mathrm{~Hz}$, $1 \mathrm{H})$, 7.34-7.38 (m, 3H), 7.46-7.51 (m, 2H). ${ }^{11} \mathbf{B}$ NMR (128 $\left.\mathbf{~ M H z}, \mathbf{C D C l}_{3}\right): \delta 15.2 .{ }^{13} \mathbf{C}$ NMR (100 Et $\left.\quad \mathbf{M H z}, \mathbf{C D C l}_{3}\right): \delta 14.4,17.6,25.3,36.9,79.2,80.6,96.8,115.3,122.2,128.5,128.9,131.7,172.1$. The carbon directly attached to the boron atom was not detected, likely due to quadrupolar relaxation. IR (ATR-IR): 3396, 2976, 2293, 1642, 1526, 1361, 1299, 1248, 1161, 1134, 1081, 1051, 871, 812, 753, 713, 688, 667, 602, 570, 523, $509 \mathrm{~cm}^{-1}$. HRMS (ESI): $m / z$ calculated for $\mathrm{C}_{20} \mathrm{H}_{27} \mathrm{BNO}_{3}{ }^{+}[\mathrm{M}+\mathrm{H}]^{+}=340.2079$, found: 340.2078. mp: $177.9-178.9{ }^{\circ} \mathrm{C}$

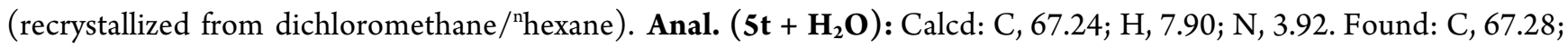
$\mathrm{H}, 7.92 ; \mathrm{N}, 3.85$.

(E)-N-ethyl-5-methoxy-2-(phenyl(4,4,5,5-tetramethyl-1,3,2-dioxaborolan-2-yl)methylene)pent-3-ynamide (6a)

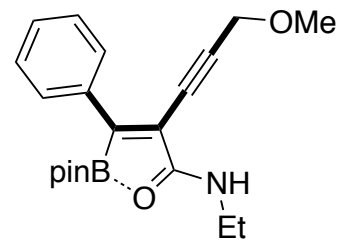

Following the General Procedure, the titled compound was obtained as a colorless solid in $25 \%$ yield $(24.7 \mathrm{mg})$ after purification using the recycling preparative HPLC. ${ }^{1} \mathbf{H}$ NMR (400 MHz, $\left.\mathbf{C D C l}_{3}\right): \delta 1.26(\mathrm{~s}, 12 \mathrm{H}), 1.31(\mathrm{t}, J=7.3 \mathrm{~Hz}, 3 \mathrm{H}), 3.37(\mathrm{~s}, 3 \mathrm{H}), 3.60(\mathrm{dq}, J=6.8,7.3 \mathrm{~Hz}$, $2 \mathrm{H}), 4.28(\mathrm{~s}, 2 \mathrm{H}), 6.67$ (br t, $J=6.8 \mathrm{~Hz}, 1 \mathrm{H}), 7.33-7.39(\mathrm{~m}, 3 \mathrm{H}), 7.87(\mathrm{~d}, J=7.6 \mathrm{~Hz}, 2 \mathrm{H}) .{ }^{11} \mathbf{B}$ NMR (128 MHz, $\mathbf{C D C l} 3): \delta 15.4 .{ }^{13} \mathbf{C}$ NMR (100 $\left.\mathbf{~ M H z , ~} \mathbf{C D C l}_{3}\right): \delta 14.3,25.9,31.0,37.0,57.9$, $60.4,80.8,92.4,113.0,127.8,128.5,129.2,137.3,172.0$. The carbon directly attached to the boron atom was not detected, likely due to quadrupolar relaxation. IR (ATR-IR): 3734, 2970, 2373, 2325, 1737, 1646, 1521, 1364, 1217, 1129, 1099, 937, 759, 698, 585, $507 \mathrm{~cm}^{-1}$. HRMS (ESI): $\mathrm{m} / z$ calculated for $\mathrm{C}_{21} \mathrm{H}_{29} \mathrm{BNO}_{4}{ }^{+}[\mathrm{M}+\mathrm{H}]^{+}=370.2184$, found: $370.2184 . \mathbf{m p :} 84.2-84.6{ }^{\circ} \mathrm{C}$ (recrystallized from dichloromethane/ ${ }^{\mathrm{n} h e x a n e}$ ). Anal. $\left(\mathbf{6 a}+\mathbf{9} / \mathbf{1 0} \mathbf{H}_{2} \mathbf{O}\right)$ : Calcd: C, 65.43; H, 7.79; N, 3.63. Found: C, 65.42; H, 7.65; N, 3.66. 
(E)-7-chloro- $N$-ethyl-2-(phenyl(4,4,5,5-tetramethyl-1,3,2-dioxaborolan-2-yl)methylene)hept-3-ynamide (6b)

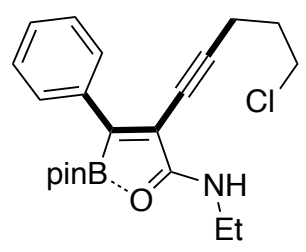

Following the General Procedure (MPLC: gradient, dichloromethane $/$ EtOAc $=93 / 9 \rightarrow 60 / 40$ ) the titled compound was obtained as a yellow solid in $34 \%$ yield $(34.2 \mathrm{mg}) .{ }^{1} \mathbf{H}$ NMR (400 MHz, $\left.\mathbf{C D C l}_{3}\right): \delta 1.26(\mathrm{~s}, 12 \mathrm{H}), 1.31(\mathrm{t}, J=7.3 \mathrm{~Hz}, 3 \mathrm{H}), 1.98-2.02(\mathrm{~m}, 2 \mathrm{H}), 2.61(\mathrm{t}, J=6.6 \mathrm{~Hz}, 2 \mathrm{H})$, 3.56-3.64 (m, 4H), $6.68(\mathrm{br} \mathrm{t}, J=5.6 \mathrm{~Hz}, 1 \mathrm{H}), 7.32-7.39(\mathrm{~m}, 3 \mathrm{H}), 7.86(\mathrm{~d}, J=7.6 \mathrm{~Hz}, 2 \mathrm{H}) .{ }^{11} \mathbf{B}$ NMR (128 MHz, $\left.\mathbf{C D C l}_{3}\right): \delta 20.5 .{ }^{13} \mathbf{C}$ NMR (100 MHz, $\left.\mathbf{C D C l}_{3}\right): \delta 14.3,17.0,25.9,30.4,36.9$, $43.6,73.7,80.7,95.7,113.9,127.7,128.5,129.0,137.6,172.4$. The carbon directly attached to the boron atom was not detected, likely due to quadrupolar relaxation. IR (ATR-IR): 2970, 2932, 2373, 2326, 1736, 1637, 1507, 1444, 1363, 1174, 1126, 1032, 975, 933, 897, 760, 724, 698, 662, 601, $505 \mathrm{~cm}^{-1}$. HRMS (ESI): $m / z$ calculated for $\mathrm{C}_{22} \mathrm{H}_{30} \mathrm{BClNO}_{3}{ }^{+}[\mathrm{M}+\mathrm{H}]^{+}=402.2002$, found: 402.2002. mp: 62.4-62.5 ${ }^{\circ} \mathrm{C}$ (recrystallized from dichloromethane $\left./{ }^{\mathrm{n} h e x a n e}\right)$. Anal. $\left(\mathbf{6 b}+\mathbf{9} / \mathbf{1 0} \mathbf{H}_{2} \mathbf{O}\right)$ : Calcd: C, 63.22; H, 7.43; N, 3.35. Found: C, 663.29; H, 7.44; N, 3.42 .

\section{2-6. Transformations of Products (Scheme 4)}

$(2 R, 3 R, 4 S, 5 R, 6 R)-2-($ acetoxymethyl)-6-(2-(4- (( $(E)-4-p h e n y l-2-(p h e n y l(4,4,5,5$-tetramethyl-1,3,2-dioxaborolan -2-yl)methylene)but-3-ynamido)methyl)-1H-1,2,3-triazol-1-yl)ethoxy)tetrahydro-2H-pyran-3,4,5-triyl triacetate $(7)$
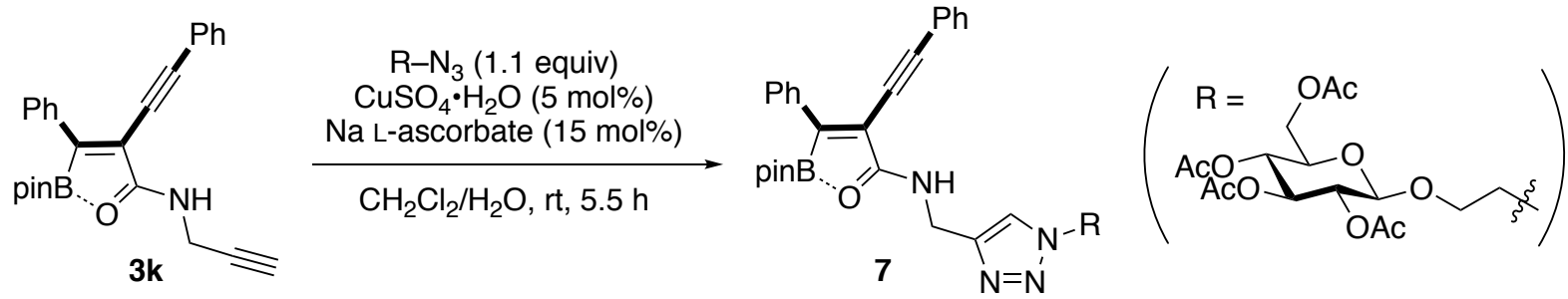

Following the protocol by Kim et al., ${ }^{14}$ to a test tube were added azide reagent $(45.9 \mathrm{mg}, 0.11 \mathrm{mmol})$, dichloromethane (1 $\mathrm{mL}$ ), 3k (41.1 mg, $0.1 \mathrm{mmol}), \mathrm{CuSO}_{4} \cdot \mathrm{H}_{2} \mathrm{O}(1.3 \mathrm{mg}, 5 \mathrm{~mol} \%)$, sodium L-ascorbate $(3.0 \mathrm{mg}, 15 \mathrm{~mol} \%)$, and water $(1 \mathrm{~mL})$. The resultant suspension was stirred at $\mathrm{rt}$ for $3 \mathrm{~h}$. The organic layer was extracted with EtOAc three times. The combined organic layer was dried over $\mathrm{Na}_{2} \mathrm{SO}_{4}$, filtered, and concentrated on a rotary evaporator. The crude material was purified by MPLC: gradient, dichloromethane/EtOAc $=20 / 80 \rightarrow 0 / 100)$. The product 7 was obtained as colorless sticky oil in 90\% (82.9 mg). ${ }^{1} \mathbf{H}$ NMR (400 MHz, $\left.\mathbf{C D C l}_{3}\right): \delta 1.31$ (s, 12H), $1.94(\mathrm{~s}, 3 \mathrm{H}), 1.95$ (s, 3H), $2.02(\mathrm{~s}, 3 \mathrm{H}), 2.08(\mathrm{~s}, 3 \mathrm{H})$, $3.69(\mathrm{br} \mathrm{d}, J=8.8 \mathrm{~Hz}, 1 \mathrm{H}), 3.97$ (br dd, $J=6.8,8.8 \mathrm{~Hz}, 1 \mathrm{H}), 4.10(\mathrm{~d}, J=12.2 \mathrm{~Hz}, 1 \mathrm{H}), 4.22-4.25(\mathrm{~m}, 2 \mathrm{H}), 4.48(\mathrm{~d}, J=8.0$ $\mathrm{Hz}, 1 \mathrm{H}), 4.52-4.55(\mathrm{~m}, 1 \mathrm{H}), 4.63(\mathrm{br} \mathrm{d}, J=14.0 \mathrm{~Hz}, 1 \mathrm{H}), 4.82(\mathrm{dd}, J=4.7,15.5 \mathrm{~Hz}, 1 \mathrm{H}), 4.88-4.93(\mathrm{~m}, 1 \mathrm{H}), 4.96(\mathrm{dd}, J$ $=8.4,9.0 \mathrm{~Hz}, 1 \mathrm{H}), 5.06(\mathrm{dd}, J=9.6,9.6 \mathrm{~Hz}, 1 \mathrm{H}), 5.17(\mathrm{dd}, J=9.6,9.6 \mathrm{~Hz}, 1 \mathrm{H}), 7.35-7.45(\mathrm{~m}, 6 \mathrm{H}), 7.40(\mathrm{~d}, J=7.4 \mathrm{~Hz}$, 2H), 7.65 (s, $1 \mathrm{H}), 7.77$ (br s, $1 \mathrm{H}), 7.99(\mathrm{~d}, J=7.4 \mathrm{~Hz}, 2 \mathrm{H}) .{ }^{11} \mathbf{B}$ NMR (128 $\left.\mathbf{~ M H z}, \mathbf{C D C l}_{3}\right): \delta 14.8 \cdot{ }^{13} \mathbf{C} \mathbf{~ N M R}(\mathbf{1 0 0} \mathbf{~ M H z}$, $\left.\mathbf{C D C l}_{3}\right): \delta 20.5,20.6,20.7,20.8,26.0,37.4,50.1,61.7,67.6,68.2,71.0,72.0,72.3,80.9,81.2,96.9,100.5,112.0,122.1$, $124.0,127.8,128.5,128.9,129.0,129.3,131.7,137.5,142.0,169.4,169.4,170.1,170.6,172.1$. The carbon directly attached to the boron atom was not detected, likely due to quadrupolar relaxation. IR (ATR-IR): 3734, 3649, 2971, 2374, 2326, 1749, 1635, 1508, 1371, 1363, 1276, 1260, 1218, 1129, 1055, 973, 752, 689, 573, $529 \mathrm{~cm}^{-1}$. HRMS (ESI): $m / z$ calculated for $\mathrm{C}_{42} \mathrm{H}_{50} \mathrm{BN}_{4} \mathrm{O}_{13}{ }^{+}[\mathrm{M}+\mathrm{H}]^{+}=829.3462$, found: 829.3463. Anal. $\left(7+\mathbf{1} / \mathbf{2} \mathbf{H}_{2} \mathbf{O}+\right.$ 
dichloromethane): Calcd: C, 56.42; H, 5.81; N, 5.98. Found: C, 56.38; H, 5.59; N, 6.17.

\section{(E)-2-((difluoroboraneyl)(phenyl)methylene)-N-ethyl-4-phenylbut-3-ynamide (8)}

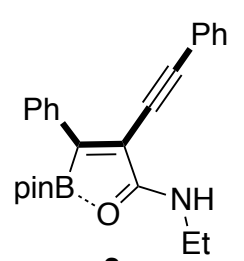

3a

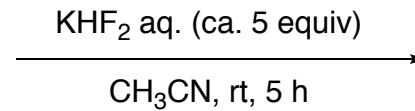

$\mathrm{CH}_{3} \mathrm{CN}, \mathrm{rt}, 5 \mathrm{~h}$

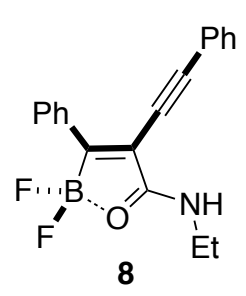

Following the procedure by Molander et al., ${ }^{15}$ the compound $3 \mathrm{a}(100 \mathrm{mg}, 0.25 \mathrm{mmol})$ was dissolved in $\mathrm{CH}_{3} \mathrm{CN}(1.4 \mathrm{~mL})$ and aqueous $\mathrm{KHF}_{2}$ (ca. $4.2 \mathrm{M}, 0.18 \mathrm{~mL}$, ca. 3.0 eq.) was added to the solution under stirring. After $1 \mathrm{~h}$, additional portion of $\mathrm{KHF}_{2}$ (39.3 mg, 2.0 eq.) was added and stirring was continued for $4 \mathrm{~h}$ at $\mathrm{rt}$. $\mathrm{H}_{2} \mathrm{O}$ was added to the mixture and the product was extracted with $\mathrm{Et}_{2} \mathrm{O}$ three times. The combined organic layer was washed with brine and dried over $\mathrm{Na}_{2} \mathrm{SO}_{4}$, filtered, and concentrated on a rotary evaporator. The crude material was purified by MPLC (gradient, hexane $/$ EtOAc $=$ 8/5). The desired product 8 was obtained as a colorless solid in 39\% (31.2 mg). ${ }^{1} \mathbf{H} \mathbf{~ N M R}\left(\mathbf{5 0 0} \mathbf{~ M H z}, \mathbf{C D C l}_{3}\right): \delta 1.41(\mathrm{t}$, $J=7.3 \mathrm{~Hz}, 3 \mathrm{H}), 3.73(\mathrm{dq}, J=7.3,0.9 \mathrm{~Hz}, 2 \mathrm{H}), 7.04(\mathrm{br} \mathrm{s}, 1 \mathrm{H}), 7.40-7.48(\mathrm{~m}, 6 \mathrm{H}), 7.54-7.56(\mathrm{~m}, 2 \mathrm{H}), 8.21-8.23(\mathrm{~m}, 2 \mathrm{H})$.

${ }^{11}$ B NMR (128 MHz, $\mathbf{C D C l}_{3}$ ): $\delta$ 8.76. ${ }^{13} \mathbf{C}$ NMR (125 $\left.\mathbf{~ M H z , ~} \mathbf{C D C l}_{3}\right): \delta 14.3,37.9,80.3,99.2,109.3,121.5,128.6,128.7$, $129.6,130.7,131.6,131.7,134.8,174.8$. The carbon directly attached to the boron atom was not detected, likely due to quadrupolar relaxation. ${ }^{19} \mathbf{F}$ NMR (470 $\left.\mathbf{~ M H z}, \mathbf{C D C l}_{3}\right): \delta$-150.7. IR (ATR-IR): 3334, 3302, 3065, 2989, 2935, 2359, $1665,1646,1496,1445,1339,1280,1239,1156,1119,1067,1029,946,923,819,778,753,709,690,648,578,531,489$, 467, 447, 435, 418, $404 \mathrm{~cm}^{-1}$. HRMS (ESI): $\mathrm{m} / z$ calculated for $\mathrm{C}_{19} \mathrm{H}_{15} \mathrm{BF}_{2} \mathrm{NO}^{-}[\mathrm{M}-\mathrm{H}]^{-}=322.1220$, found: 322.1234 . mp: $136.6-142.2{ }^{\circ} \mathrm{C}$ (recrystallized from $\mathrm{Et}_{2} \mathrm{O}$ ). Anal. $\left(\mathbf{8}+\mathbf{1} / \mathbf{8} \mathbf{H}_{2} \mathbf{O}\right)$ : Calcd: $\mathrm{C}, 70.13 ; \mathrm{H}, 5.03 ; \mathrm{N}, 4.30$. Found: $\mathrm{C}$, 70.14; H, 5.26; N, 4.20 .

\section{2-7. Computational Studies (Figure 4)}

All DFT calculations were carried with the Gaussian 16 program package. ${ }^{16}$ The molecular structures optimizations were conducted at the $\mathrm{B}^{2} \mathrm{LYP}^{17}$ level in the gas phase using $6-31+\mathrm{G}^{*}$ basis set for all the atoms. The vibrational frequencies were computed at the same level. The Cartesian coordination of the optimized structure of $\mathbf{5} \mathbf{f}$ is shown below.

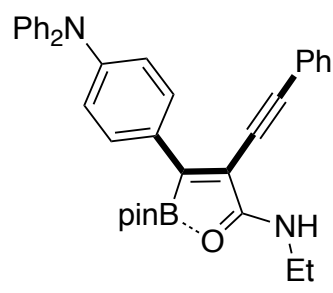

Energy (RB3LYP): -1595.75205416

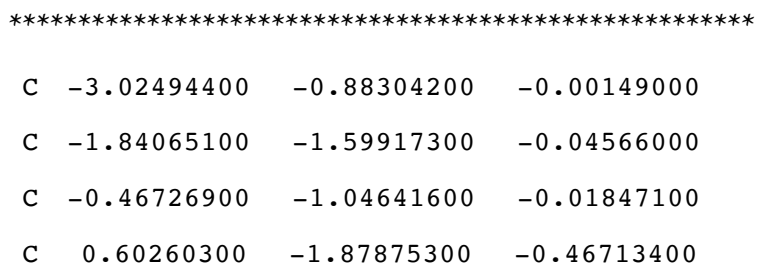

$\begin{array}{lrrr}\text { C } & -0.14437300 & 0.27024200 & 0.42542000 \\ \text { C } & 1.92455200 & -1.42310500 & -0.48536400 \\ \text { H } & 0.34282800 & -2.88136300 & -0.84323900 \\ \text { C } & 1.17749500 & 0.72780200 & 0.43353900 \\ \text { H } & -0.94618300 & 0.92600300 & 0.78951200 \\ \text { C } & 2.23580200 & -0.11018700 & -0.02598400 \\ \text { H } & 2.73037000 & -2.06829200 & -0.85593900 \\ \text { H } & 1.41243300 & 1.73442100 & 0.80009200 \\ \text { C } & -3.31266700 & 0.52299800 & 0.09037100 \\ \text { C } & -3.62240900 & 1.69909800 & 0.16097200 \\ \text { O } & -3.83871600 & -3.13441800 & -0.26316800\end{array}$




\begin{tabular}{|c|c|c|c|}
\hline & 3.96037700 & 3.10741400 & 0.24632500 \\
\hline & 2.93440800 & .09753700 & .25187400 \\
\hline & -5.31915300 & .53102300 & .32603300 \\
\hline & -3.26234100 & .46156600 & .33473900 \\
\hline & -1.88414800 & .78251800 & .18964200 \\
\hline & -5.63561400 & .89771700 & .40847000 \\
\hline & -6.11953800 & 2.77936100 & 0.32352000 \\
\hline & -4.61099600 & 5.86657600 & 0.41321000 \\
\hline & -2.46192300 & 6.21386500 & 0.33803900 \\
\hline $\mathrm{H}$ & -6.68694000 & 5.20998000 & 0.46967400 \\
\hline $\mathrm{H}$ & -4.86268600 & 6.93365500 & 0.47782900 \\
\hline$C$ & -1.56567800 & -5.29529900 & -0.94286600 \\
\hline$C$ & -1.69008400 & -5.34047400 & 0.63730100 \\
\hline$H$ & -2.39019400 & -5.87151800 & -1.42117700 \\
\hline $\mathrm{H}$ & -0.60278100 & -5.72308400 & -1.29819900 \\
\hline $\mathrm{H}$ & -2.54959700 & -5.97692800 & 0.94883400 \\
\hline $\mathrm{H}$ & -0.77300700 & -5.75487300 & 1.11174000 \\
\hline C & -4.15566200 & -1.87086700 & -0.13475000 \\
\hline $\mathrm{N}$ & -5.47831700 & -1.44919500 & -0.13838000 \\
\hline 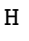 & -5.64388900 & -0.42341900 & -0.04656100 \\
\hline $\mathrm{O}$ & -1.63411900 & -3.87461500 & -1.31140100 \\
\hline O & -1.88064300 & -3.95150300 & 1.07201400 \\
\hline$B$ & -2.13268500 & -3.20793500 & -0.13634300 \\
\hline C & -6.62436500 & -2.38253600 & -0.29443600 \\
\hline $\mathrm{H}$ & -7.29552800 & -2.33102300 & 5900 \\
\hline & -7.20337600 & -2.14684500 & -1.2080650 \\
\hline
\end{tabular}

$\begin{array}{crrc}\mathrm{H} & -6.20092000 & -3.39891000 & -0.37725500 \\ \mathrm{~N} & 3.60536400 & 0.36651200 & -0.02463800 \\ \mathrm{C} & 4.70859000 & -0.56178100 & 0.20600200 \\ \mathrm{C} & 5.92565600 & -0.42068000 & -0.51368800 \\ \mathrm{C} & 4.58200600 & -1.61013600 & 1.15700000 \\ \mathrm{C} & 6.98944800 & -1.30877900 & -0.27941700 \\ \mathrm{H} & 6.02555800 & 0.38601300 & -1.25066600 \\ \mathrm{C} & 5.64947800 & -2.49903100 & 1.37070400 \\ \mathrm{H} & 3.64631200 & -1.71817900 & 1.71982700 \\ \mathrm{C} & 6.85711500 & -2.35263400 & 0.65847700 \\ \mathrm{H} & 7.92528000 & -1.19046600 & -0.84228300 \\ \mathrm{H} & 5.54005000 & -3.30581200 & 2.10811600 \\ \mathrm{H} & 7.68915800 & -3.04679200 & 0.83345500 \\ \mathrm{C} & 3.89482400 & 1.77839700 & -0.25498900 \\ \mathrm{C} & 4.93567200 & 2.42354500 & 0.46615700 \\ \mathrm{C} & 3.14698600 & 2.52211500 & -1.20763900 \\ \mathrm{C} & 5.21906900 & 3.78001400 & 0.23206500 \\ \mathrm{H} & 5.51364100 & 1.85293800 & 1.20389400 \\ \mathrm{C} & 3.43289600 & 3.88142100 & -1.42136500 \\ \mathrm{H} & 2.34839000 & 2.02534200 & -1.77275200 \\ \mathrm{C} & 4.46924500 & 4.51636100 & -0.70733700 \\ \mathrm{H} & 6.02554500 & 4.26803000 & 0.79603100 \\ \mathrm{H} & 2.84870200 & 4.44572300 & -2.16089900 \\ \mathrm{H} & 4.69168900 & 5.57680700 & -0.88255200 \\ \mathrm{H} * * * * * * * * * * * * * * * * * * * * * * * * * * * * * * * * * * * * * * * * * * * * * * * * * * \\ \mathrm{H}\end{array}$




\section{2-8. Electronic Absorption and Fluorescence Spectra (Table 1)}

- Electronic Absorption and Fluorescence Spectra of 3a (Scheme 1)
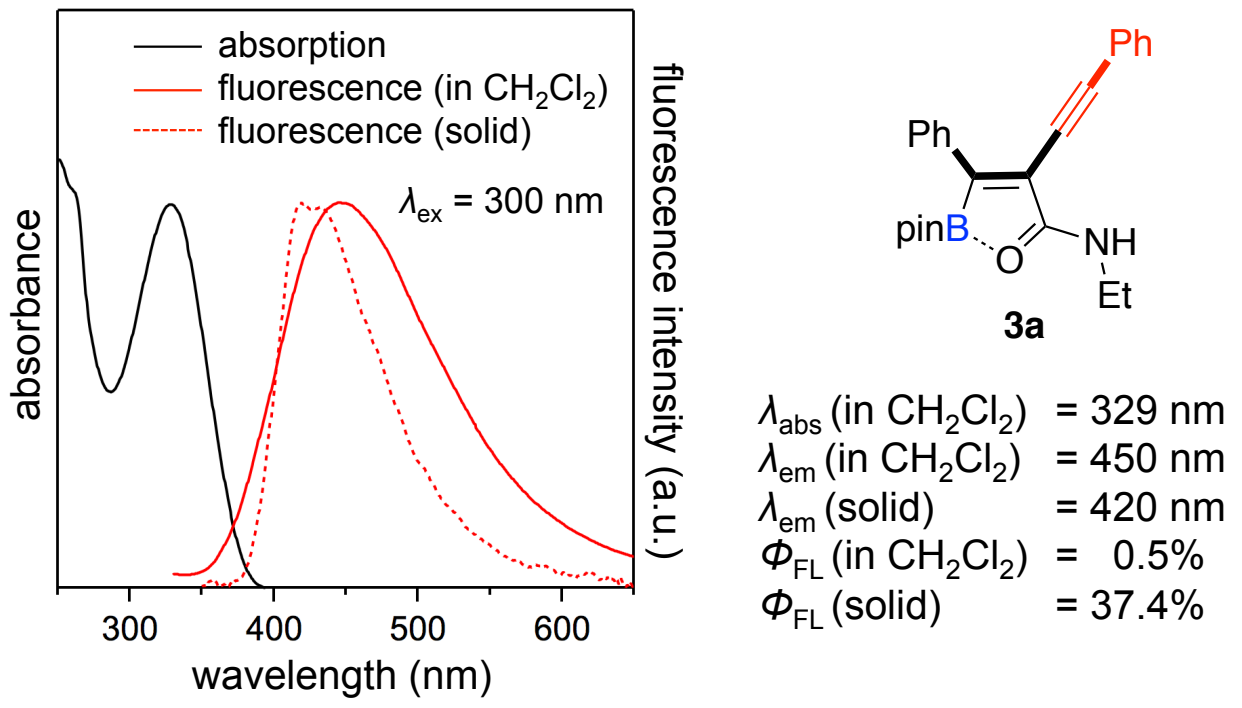

- Electronic Absorption and Fluorescence Spectra of 3a in Water/THF (Figure 3)
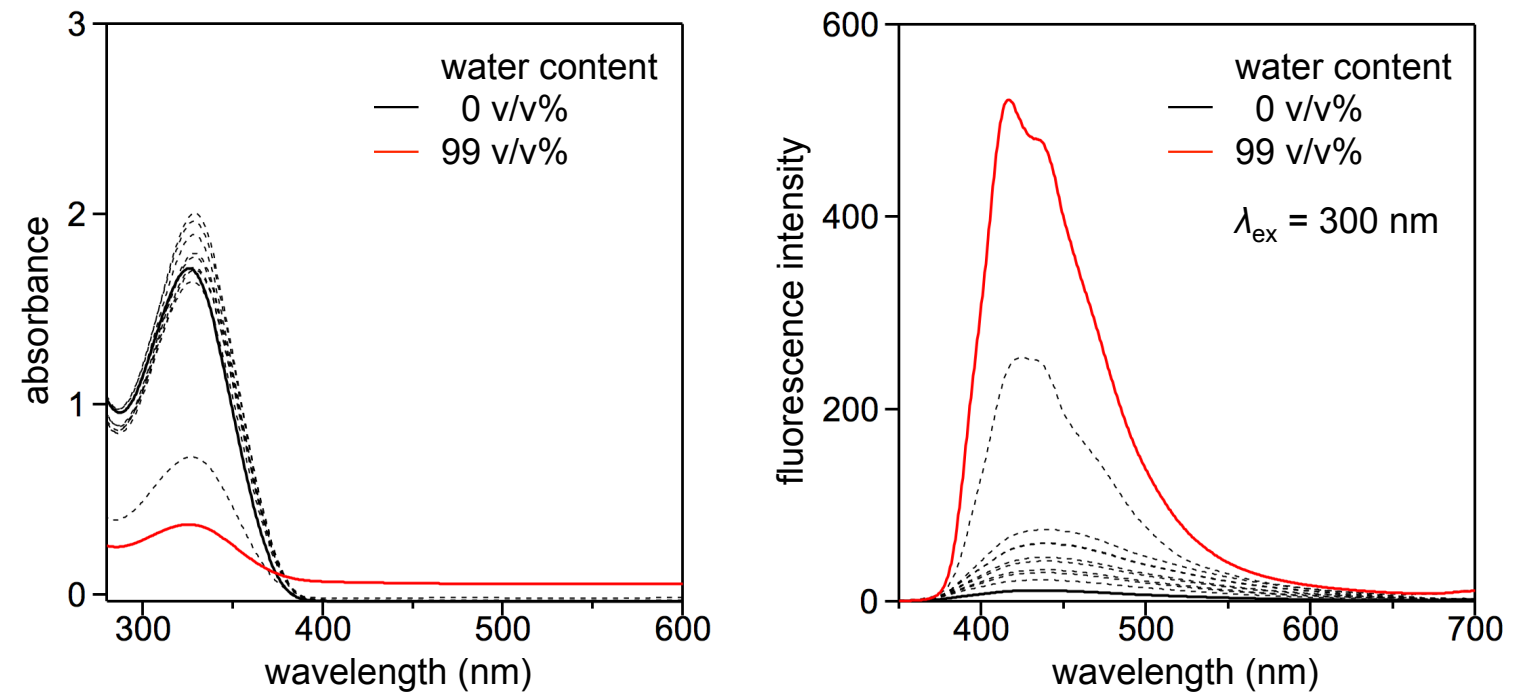
- Electronic Absorption and Fluorescence Spectra of 5t (Scheme 3)

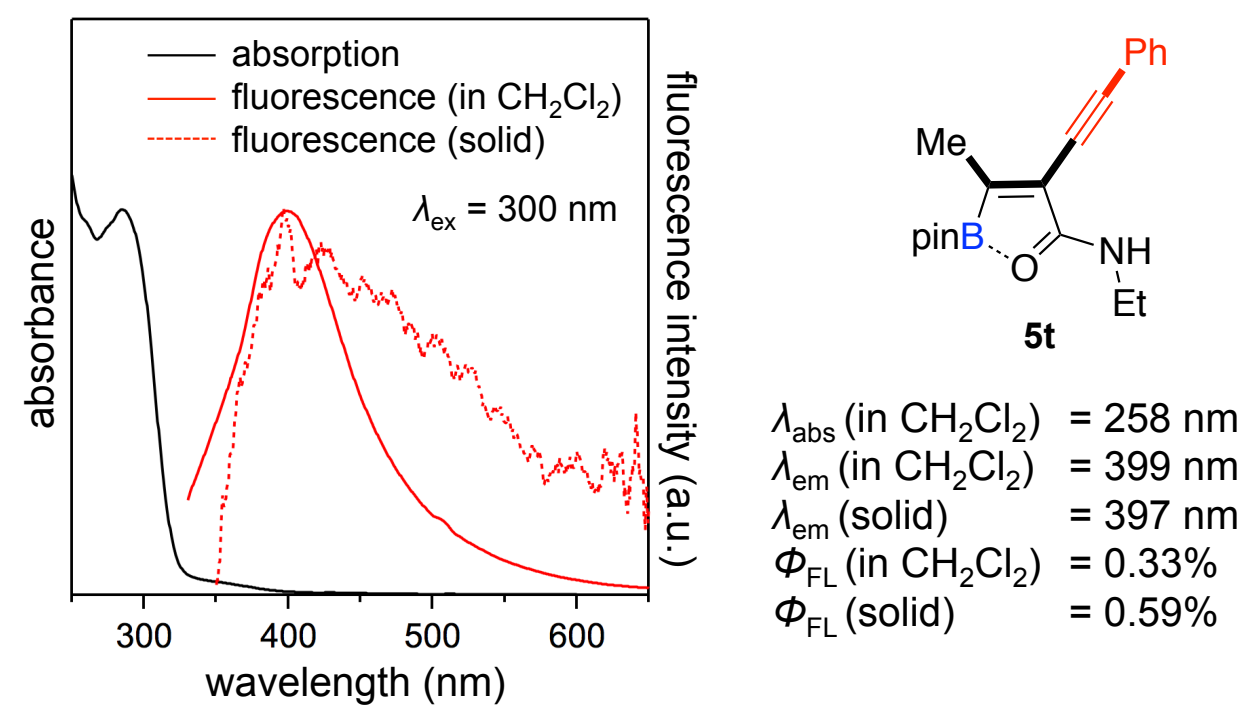


- Electronic Absorption and Fluorescence Spectra of 5a, 5h, 5d, 5g, 5f (Scheme 3)
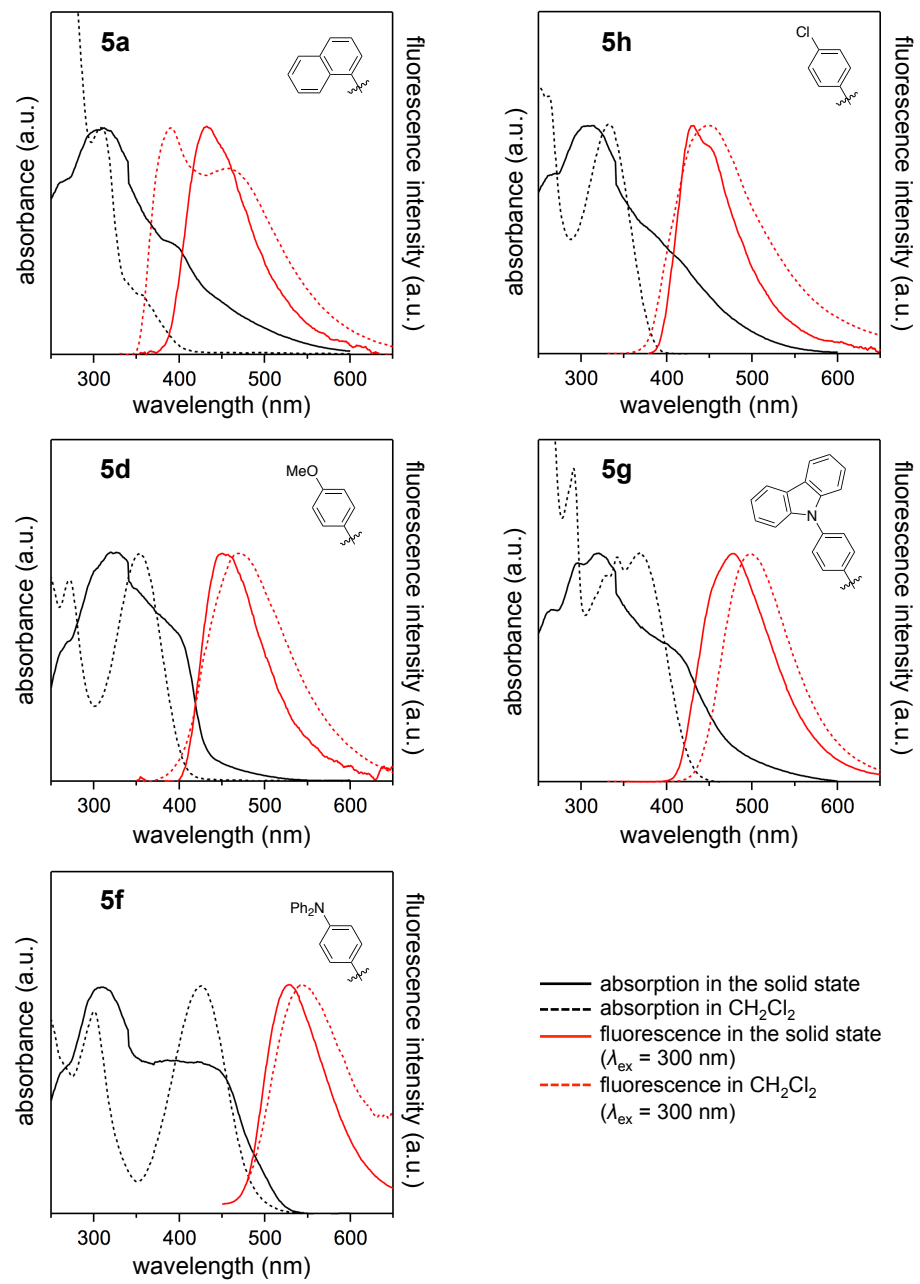

- Electronic Absorption Spectra of $5 \mathrm{f}$ (Figure 4), Y-axis was normalized at the maximum absorbance

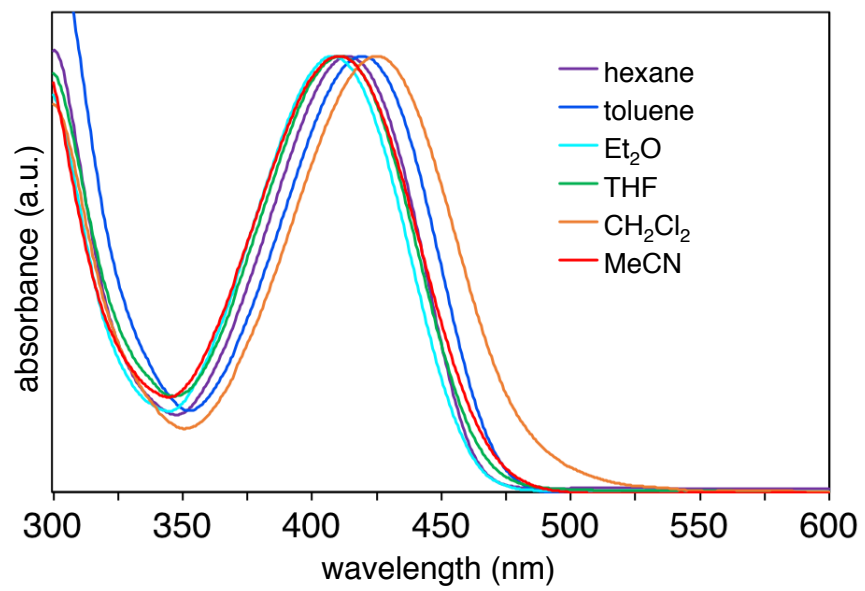




\section{References in Experimental Section}

(1) Rajapaksha, S. M.; Nlsna, T. E.; Pittman, C. U., Jr. J. Org. Chem. 2017, 82, 5678.

(2) Petrone, D. A.; Franzoni, I.; Ye, J.; Rodríguez, J. F.; Poblador-Bahamonde, A. I.; Lautens, M. J. Am. Chem. Soc. 2017, $139,3546$.

(3) Strübing, D.; Neumann, H.; Klaus, S.; Hübner, S.; Beller, M. Tetrahedron 2005, 61, 11333.

(4) Hua, H.-L.; He, Y.-T.; Qiu, Y.-F.; Li, Y.-X.; Song, B.; Gao, P.; Song, X.-R.; Guo, D.-H.; Liu, X.-Y.; Liang, Y.-M. Chem. Eur. J. 2015, 21, 1468.

(5) Barreau, M.; Ponsinet, G. Synthesis 1987, 262.

(6) Kawagoe, Y.; Moriyama, K.; Togo, H. Tetrahedron. 2013, 69, 3971.

(7) Kissane, M.; Murphy, M.; O’Brien, E.; Chopra, J.; Murphy, L.; Collins, S. G.; Lawrence, S. E.; Maguire, A. R. Org. Biomol. Chem. 2011 , 9, 2452.

(8) Strum, J. C.; Bisi, J. E.; Robert, P. J.; Gaston, R. D.; Gadwood, R. C. WO2015161285.

(9) Hassan, S.; Tschersich, R.; Müler, T. J. J. Tetrahedron Lett. 2013, 54, 4641.

(10) Zhang, Z.; Wu, W.; Liao, J.; Li, J.; Jiang, H. Chem. Eur. J. 2015, 21, 6708.

(11) Eckert T.; Ipaktshi, J. Synth. Commun. 1998, 28, 327.

(12) Parkinson, E. I.; Bair, J. S.; Cismesia, M.; Hergenrother, P. J. ACS Chem. Biol. 2013, 8, 2173.

(13) Deng, D.; Zheng, J. US 2014/0066655 A1.

(14) Lee, B.-Y.; Park, S. R.; Jeon, H. B.; Kim, K. S. Tetrahedron Lett. 2006, 47, 5105.

(15) Molander, G. A.; Yun, C.-S.; Ribagorda, M.; Biolatto, B. J. Org. Chem. 2003, 68, 5534.

(16) Gaussian 16, Revision B.01, Frisch, M. J.; Trucks, G. W.; Schlegel, H. B.; Scuseria, G. E.; Robb, M. A.; Cheeseman, J. R.; Scalmani, G.; Barone, V.; Petersson, G. A.; Nakatsuji, H.; Li, X.; Caricato, M.; Marenich, A. V.; Bloino, J.; Janesko, B. G.; Gomperts, R.; Mennucci, B.; Hratchian, H. P.; Ortiz, J. V.; Izmaylov, A. F.; Sonnenberg, J. L.; Williams-Young, D.; Ding, F.; Lipparini, F.; Egidi, F.; Goings, J.; Peng, B.; Petrone, A.; Henderson, T.; Ranasinghe, D.; Zakrzewski, V. G.; Gao, J.; Rega, N.; Zheng, G.; Liang, W.; Hada, M.; Ehara, M.; Toyota, K.; Fukuda, R.; Hasegawa, J.; Ishida, M.; Nakajima, T.; Honda, Y.; Kitao, O.; Nakai, H.; Vreven, T.; Throssell, K.; Montgomery, J. A., Jr.; Peralta, J. E.; Ogliaro, F.; Bearpark, M. J.; Heyd, J. J.; Brothers, E. N.; Kudin, K. N.; Staroverov, V. N.; Keith, T. A.; Kobayashi, R.; Normand, J.; Raghavachari, K.; Rendell, A. P.; Burant, J. C.; Iyengar, S. S.; Tomasi, J.; Cossi, M.; Millam, J. M.; Klene, M.; Adamo, C.; Cammi, R.; Ochterski, J. W.; Martin, R. L.; Morokuma, K.; Farkas, O.; Foresman, J. B.; Fox, D. J. Gaussian, Inc., Wallingford CT, 2016.

(17) a) Becke, A. D. Phys. Rev. 1988, A38, 3098; b) Becke, A. D. J. Chem. Phys. 1993, 98, 1372; c) Becke, A. D. J. Chem. Phys. 1993, 98, 5648; d) Lee, C; Yang, W.; Parr, R. G. Phys. Rev. 1988, B37, 785. 


\section{NMR Spectra}

(E)-N-ethyl-4-phenyl-2-(phenyl(4,4,5,5-tetramethyl-1,3,2-dioxaborolan-2-yl)methylene)but-3-ynamide (3a)
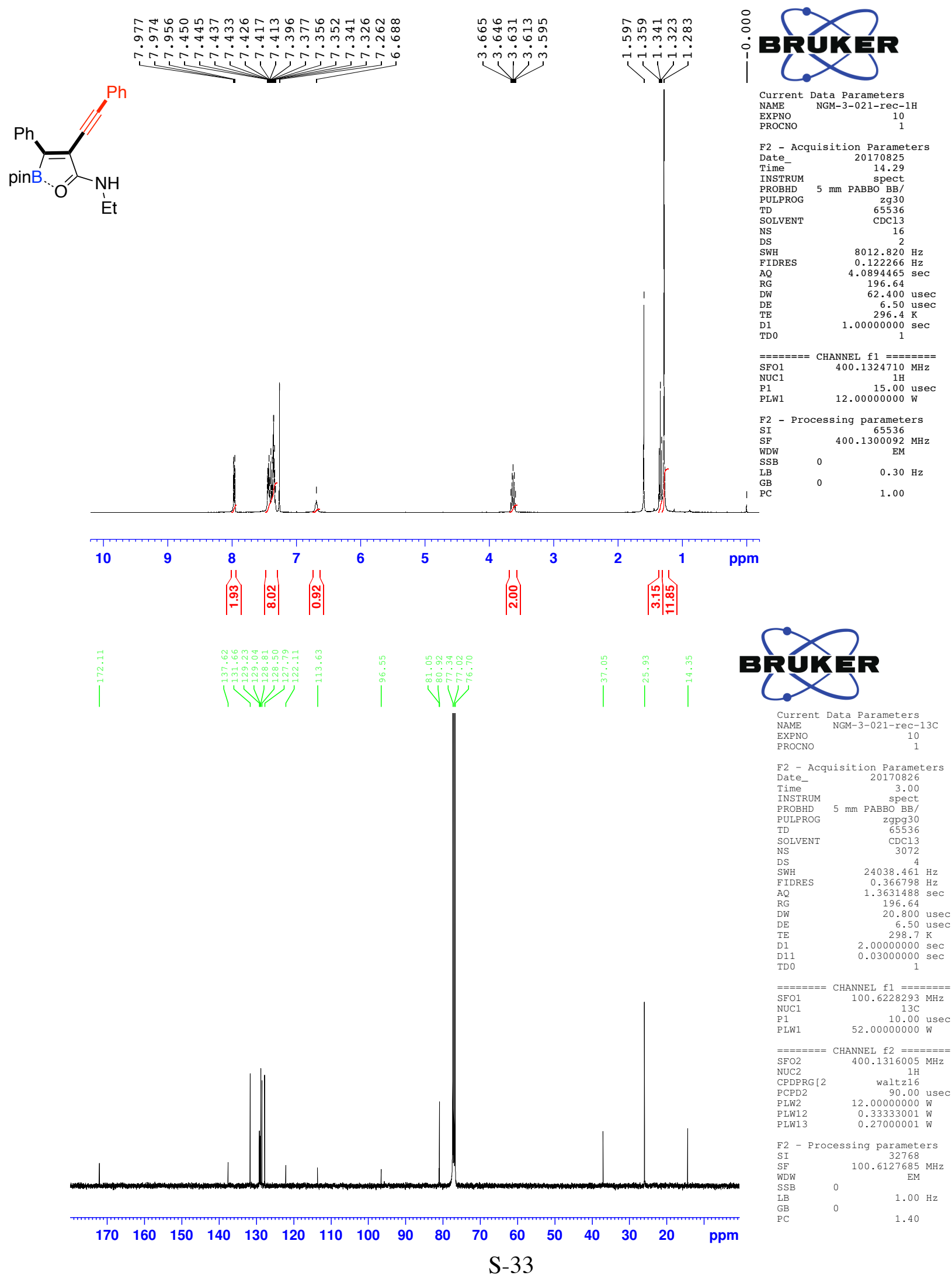

S-33 


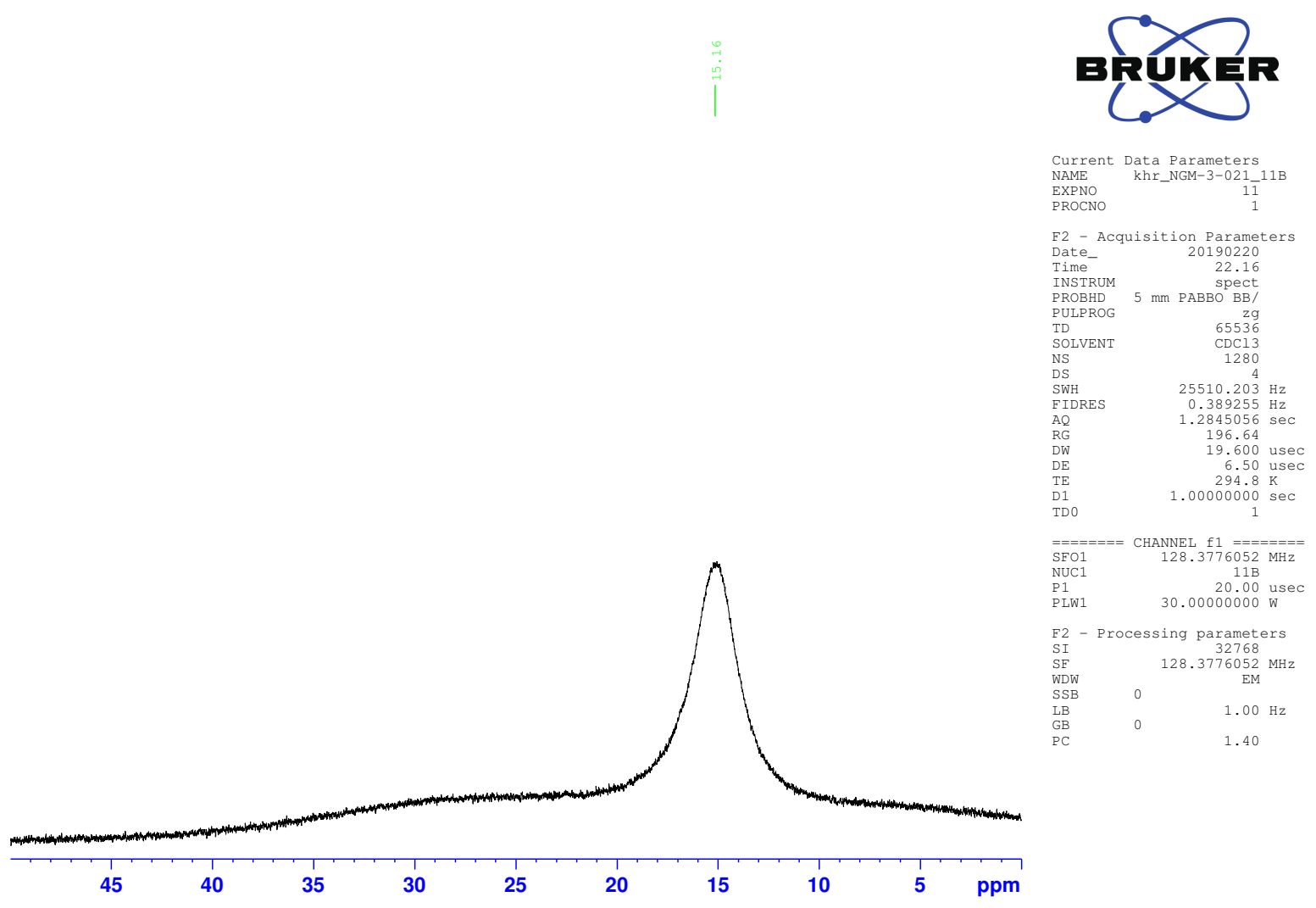

S-34 
(E)-N-methyl-4-phenyl-2-(phenyl(4,4,5,5-tetramethyl-1,3,2-dioxaborolan-2-yl)methylene)but-3-ynamide (3b)
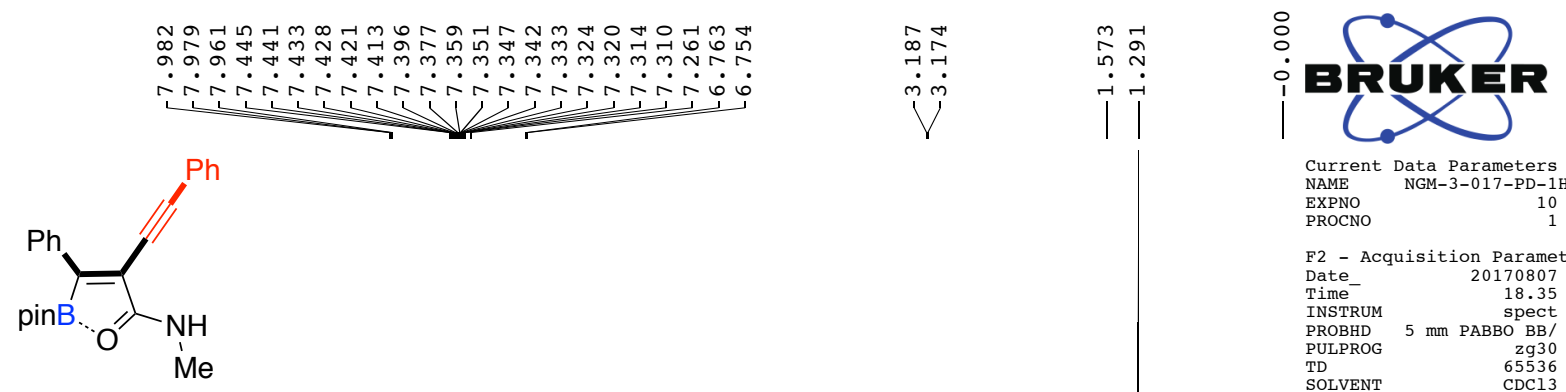

Current Data Parameters
NAME NGM-3-017-PD-1H

$\begin{array}{lr}\text { EXPNO } & 10 \\ \text { PROCNO } & 1\end{array}$

F2 - Acquisition Parameters

$\begin{array}{lr}\text { Date_- } & 20170807 \\ \text { Time } & 18.35\end{array}$

INSTRUM spect

PROBHD $5 \mathrm{~mm}$ PABBO BB/

TD

NS

SWH

SID
FIDRE
AQ
RG
DW

$\mathrm{DW}$
$\mathrm{DE}$
$\mathrm{TE}$

TE
D1
TD0

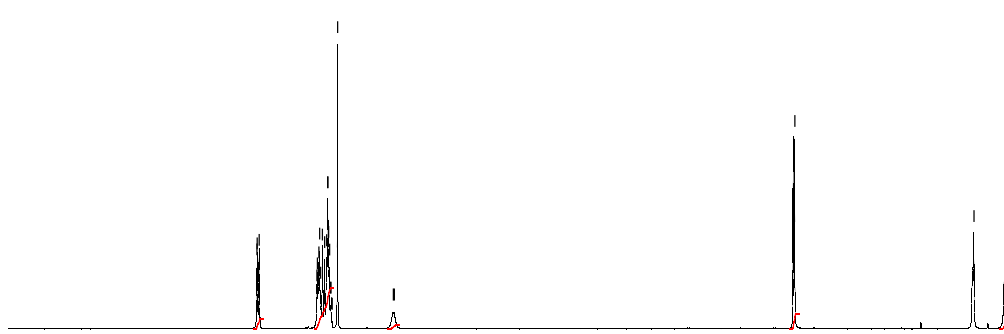

$=== \pm$
SFO1
NUC1
P1

PLW1

zg30
65536
CDC13

$8012.820 \mathrm{~Hz}$

$0.122266 \mathrm{~Hz}$

196.64

62.400 usec

6.50 usec

$1.00000000 \mathrm{sec}$

$1 \mathrm{sec}$

$400.1324710 \mathrm{MHz}$

$12.00000000 \mathrm{w}$

2 - Processing parameters

$\begin{array}{lr}\text { SI } & 65536 \\ \text { SF } & 400.1300093 \mathrm{MHz}\end{array}$

SSB $\quad 0$
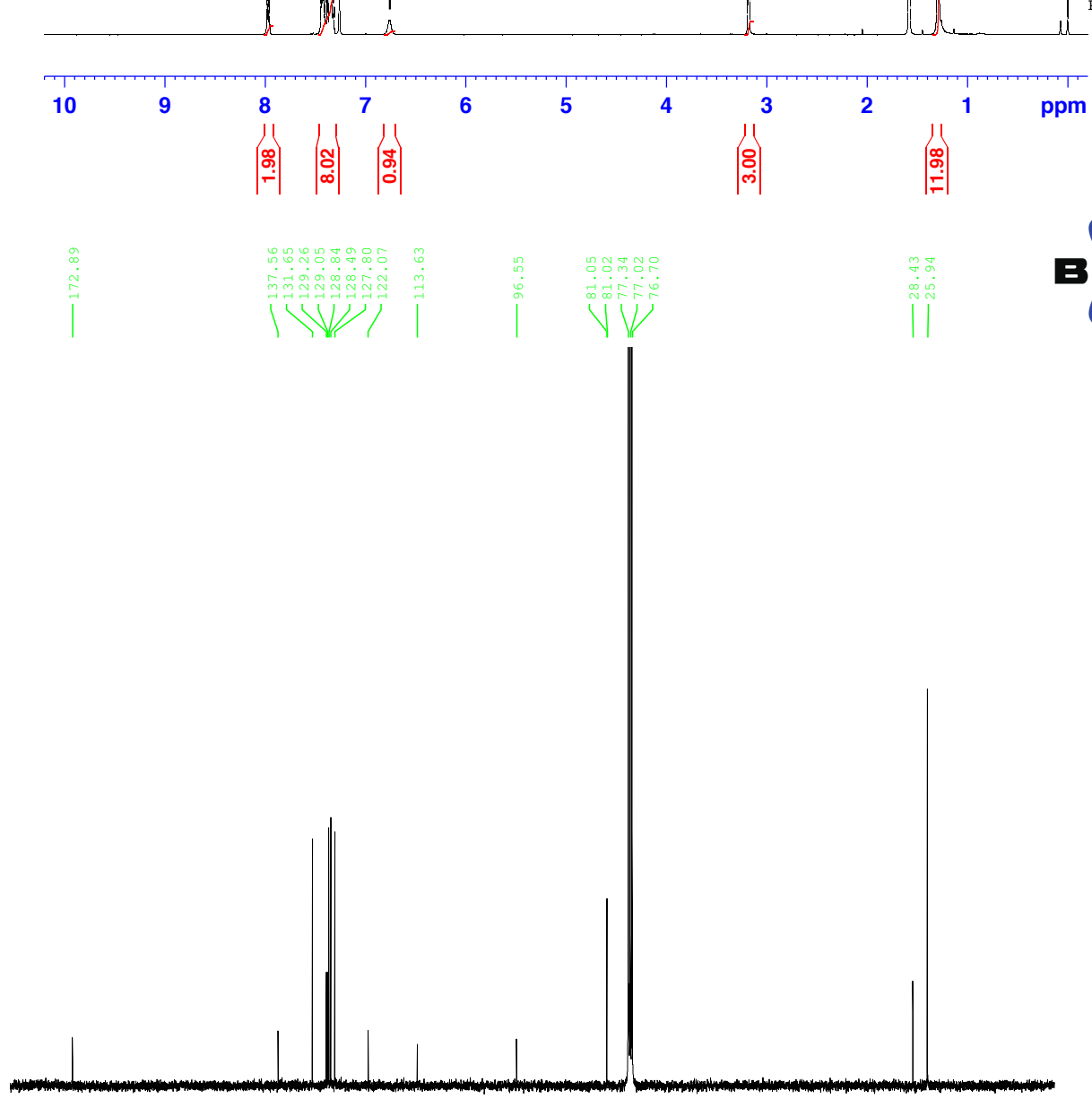

GRिRER $\begin{array}{ll}\text { Current } & \text { Data Parameters } \\ \text { NAME } & \text { NGM-3-017-PD-13C }\end{array}$

EXPNO

F2 - Acquisition Parameters $\begin{array}{lr}\text { Date_ } & 20170808 \\ \text { Time } & 3.58\end{array}$

$\begin{array}{lr}\text { Time } & 3.58 \\ \text { INSTRUM } & \text { spect }\end{array}$

Spect
PROBHD $5 \mathrm{~mm}$ PABBO BB

PROBHD
PULPROG

TD

NS

DS
SWH
FIDRES

$\begin{array}{ll}\text { AQ } & 1.3631488 \mathrm{sec} \\ \text { RG } & 1.366798 \mathrm{~Hz}\end{array}$

$\begin{array}{lr}\text { RG } & 196.64 \\ \text { DW } & 20.800 \text { usec } \\ \text { DE } & 6.50 \text { usec }\end{array}$

$\begin{array}{lr}\text { TE } & 298.8 \mathrm{~K} \\ \mathrm{D} 1 & 2.00000000 \mathrm{sec}\end{array}$

$=======$ CHANNEL f $1 \quad=======$
SFO1
$100.6228293 \mathrm{MHz}$

$\begin{array}{lr}\text { SFO1 } & 100.6228293 \mathrm{MHz} \\ \text { NUC1 } & 13 \mathrm{C}\end{array}$

P1 $\quad 10.00$ us

$=======$ CHANNEL $\mathrm{f} 2 \quad========$
$\mathrm{SFO} 2$

SFO2 400.1316005

$\begin{array}{lr}\text { NUC2 } & 1 \mathrm{H} \\ \text { CPDPRG } 2 & \text { waltz16 }\end{array}$

PCPD2 90.00 usec

$\begin{array}{lr}\text { PLW2 } & 12.00000000 \mathrm{~W} \\ \text { PLW12 } & 0.33333001 \mathrm{~W} \\ \text { PLW13 } & 0.27000001 \mathrm{~W}\end{array}$

" का

F2 - Processing parameters

$\begin{array}{ll}\text { SI } & 32768 \\ \text { SF } & 100.6127685 \\ \text { MH }\end{array}$

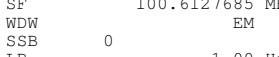

$\begin{array}{llllllllllllllllll}180 & 170 & 160 & 150 & 140 & 130 & 120 & 110 & 100 & 90 & 80 & 70 & 60 & 50 & 40 & 30 & 20 & \text { ppm }\end{array}$

\section{S-35}


(E)- $N$-isobutyl-4-phenyl-2-(phenyl(4,4,5,5-tetramethyl-1,3,2-dioxaborolan-2-yl)methylene)but-3-ynamide (3c)

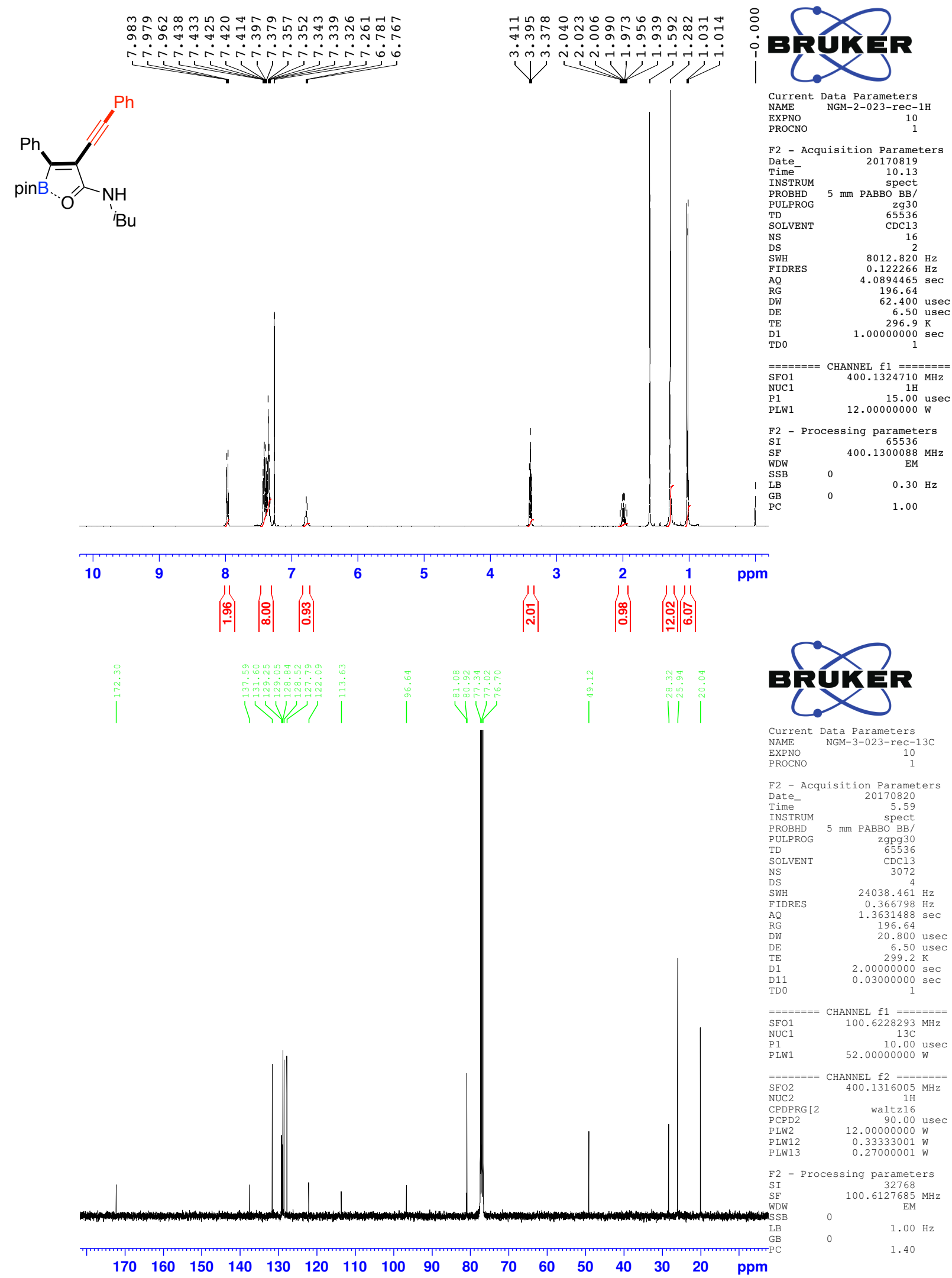

S-36 
(E)-N-benzyl-4-phenyl-2-(phenyl(4,4,5,5-tetramethyl-1,3,2-dioxaborolan-2-yl)methylene)but-3-ynamide (3d)
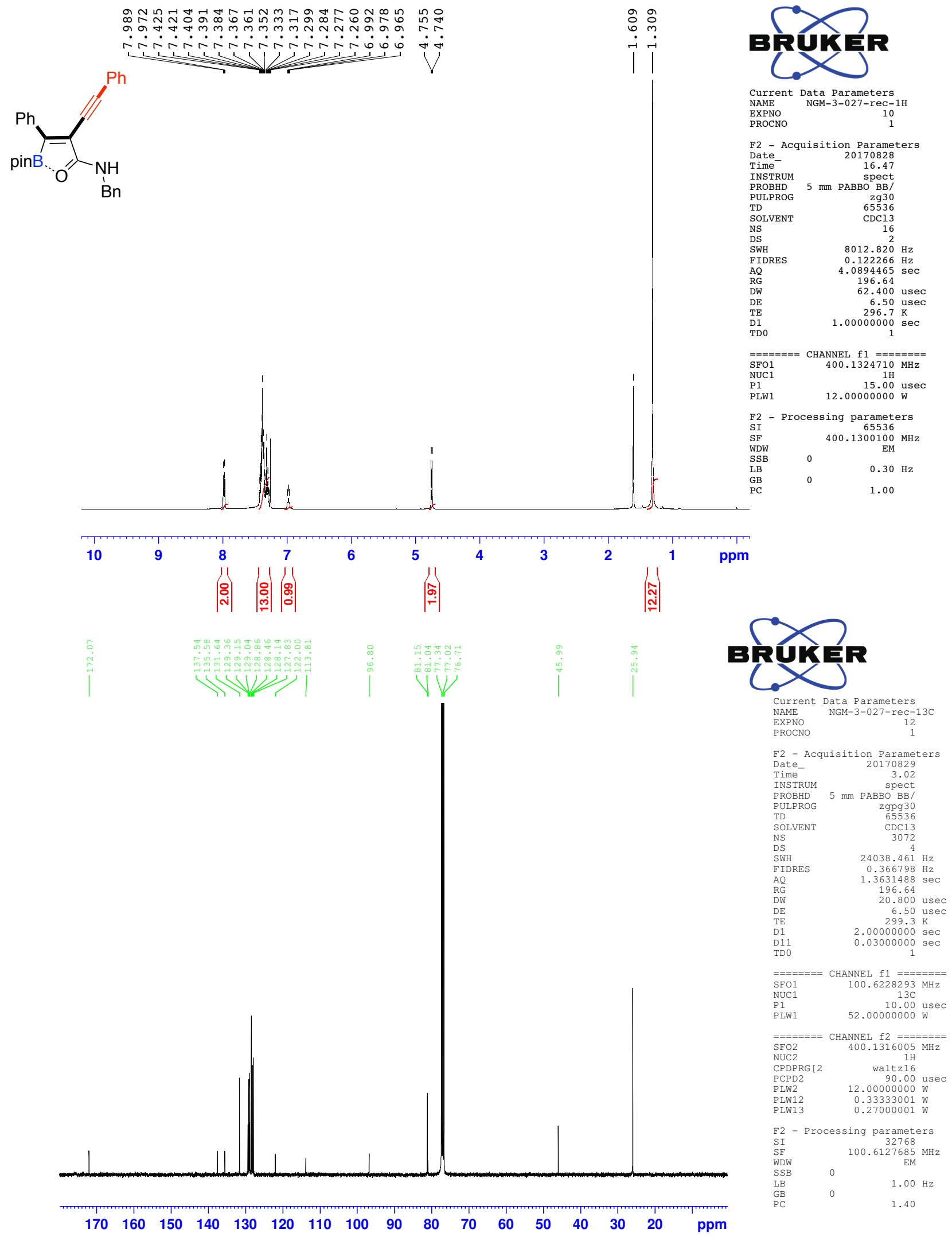

S-37 
(E)-N-isopropyl-4-phenyl-2-(phenyl(4,4,5,5-tetramethyl-1,3,2-dioxaborolan-2-yl)methylene)but-3-ynamide (3e)

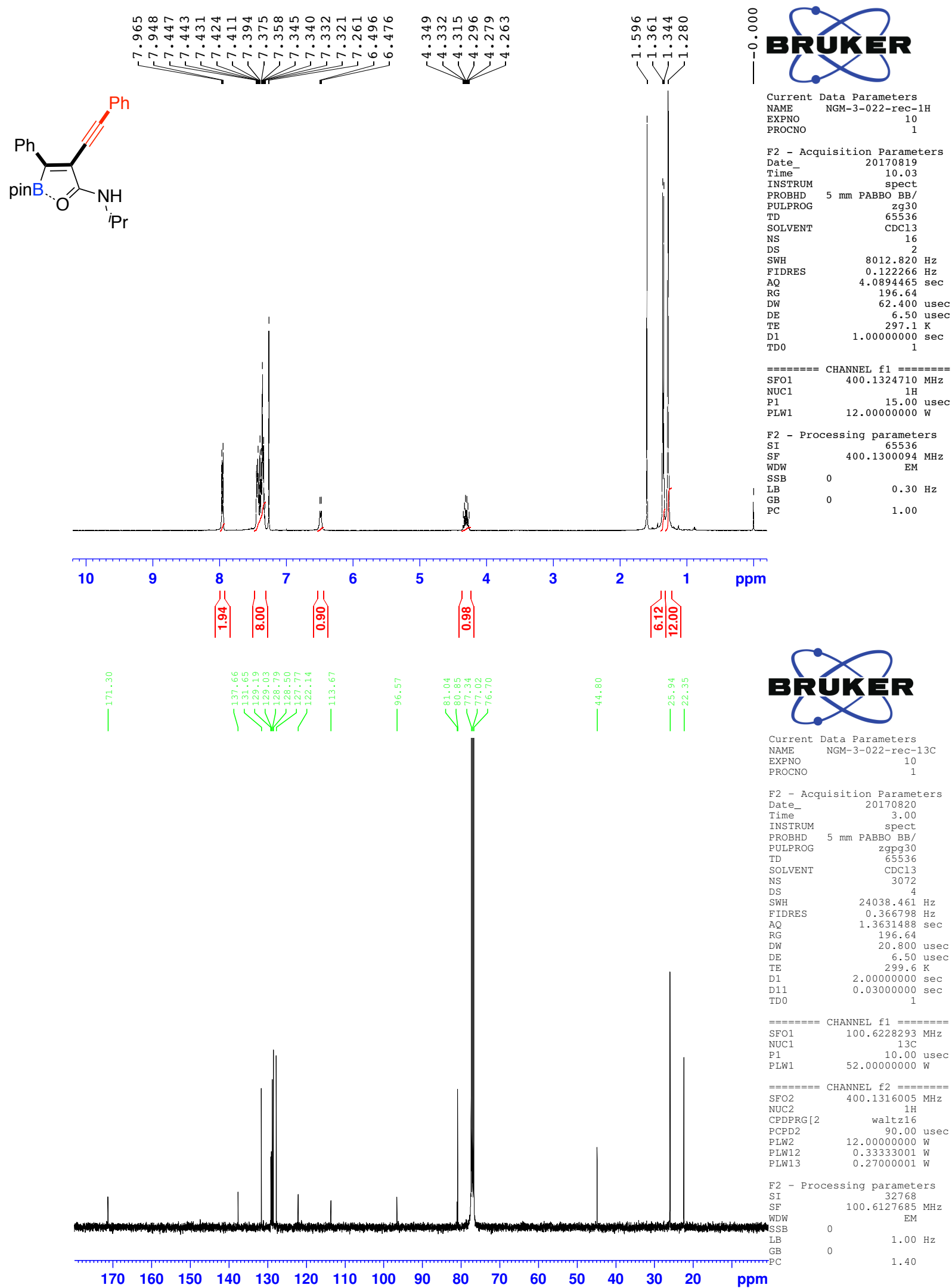

S-38 
(E)-N-cyclopropyl-4-phenyl-2-(phenyl(4,4,5,5-tetramethyl-1,3,2-dioxaborolan-2-yl)methylene)but-3-ynamide (3f)
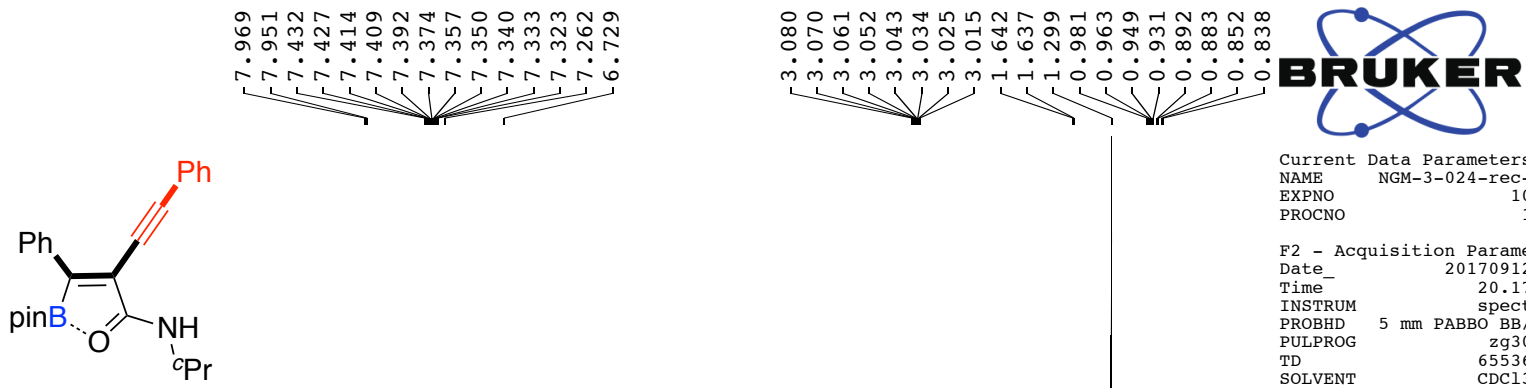

Current Data Parameters $\begin{array}{ll}\text { NAME } & \text { NGM-3-024-rec-1 } \\ \text { EXPNO } & 10\end{array}$

F2 - Acquisition Parameters

$\begin{array}{lr}\text { Date_- } & 20170912 \\ \text { Time } & 20.17\end{array}$

spect

PULPROG 5 TI

$\mathrm{zg} 30$
65536
$\mathrm{CDC} 13$

DS

$\begin{array}{ll}\text { SWH } & 8012.820 \mathrm{~Hz} \\ \text { FIDRES } & 0.122266 \mathrm{~Hz}\end{array}$

AQ $\quad 4.0894465 \mathrm{sec}$

$\begin{array}{lr}\text { RG } & 196.64 \mathrm{u} \\ \text { DW } & 62.400 \text { usec }\end{array}$

$\begin{array}{lr}\mathrm{DE} & 6.50 \mathrm{usec} \\ \mathrm{TE} & 297.0 \mathrm{~K}\end{array}$

$\begin{array}{lr}\text { TE } & 297.0 \mathrm{~K} \\ \text { D1 } & 1.00000000 \mathrm{sec}\end{array}$

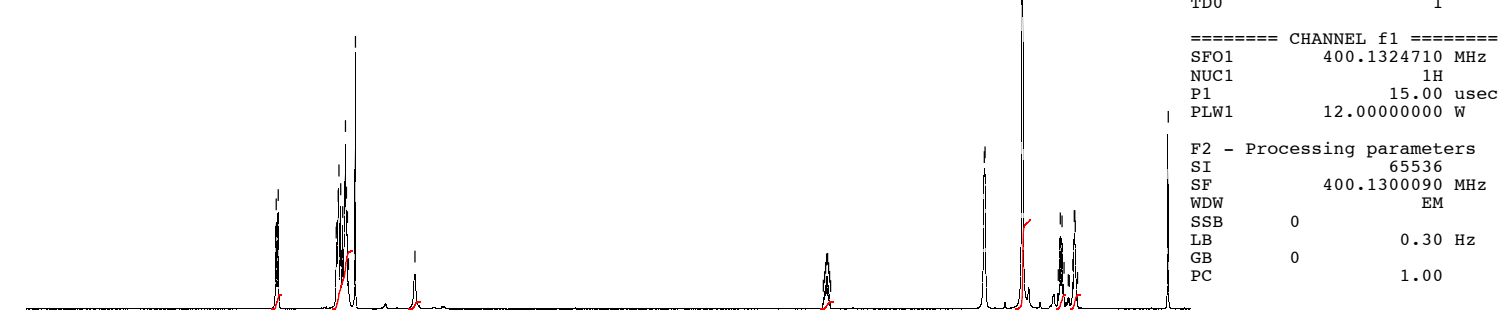

\begin{tabular}{|c|c|c|c|c|c|c|c|c|c|c|}
\hline 10 & 9 & $\begin{array}{l}8 \\
\text { के } \\
\end{array}$ & $\left|\begin{array}{l}0 \\
0 \\
\infty\end{array}\right|$ & $\begin{array}{l}7 \\
\left|\begin{array}{l}\text { o } \\
\hdashline\end{array}\right|\end{array}$ & 6 & 5 & 4 & $\begin{array}{l}3 \\
\left|\begin{array}{|}8 \\
0\end{array}\right|\end{array}$ & 2 & 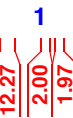 \\
\hline
\end{tabular}
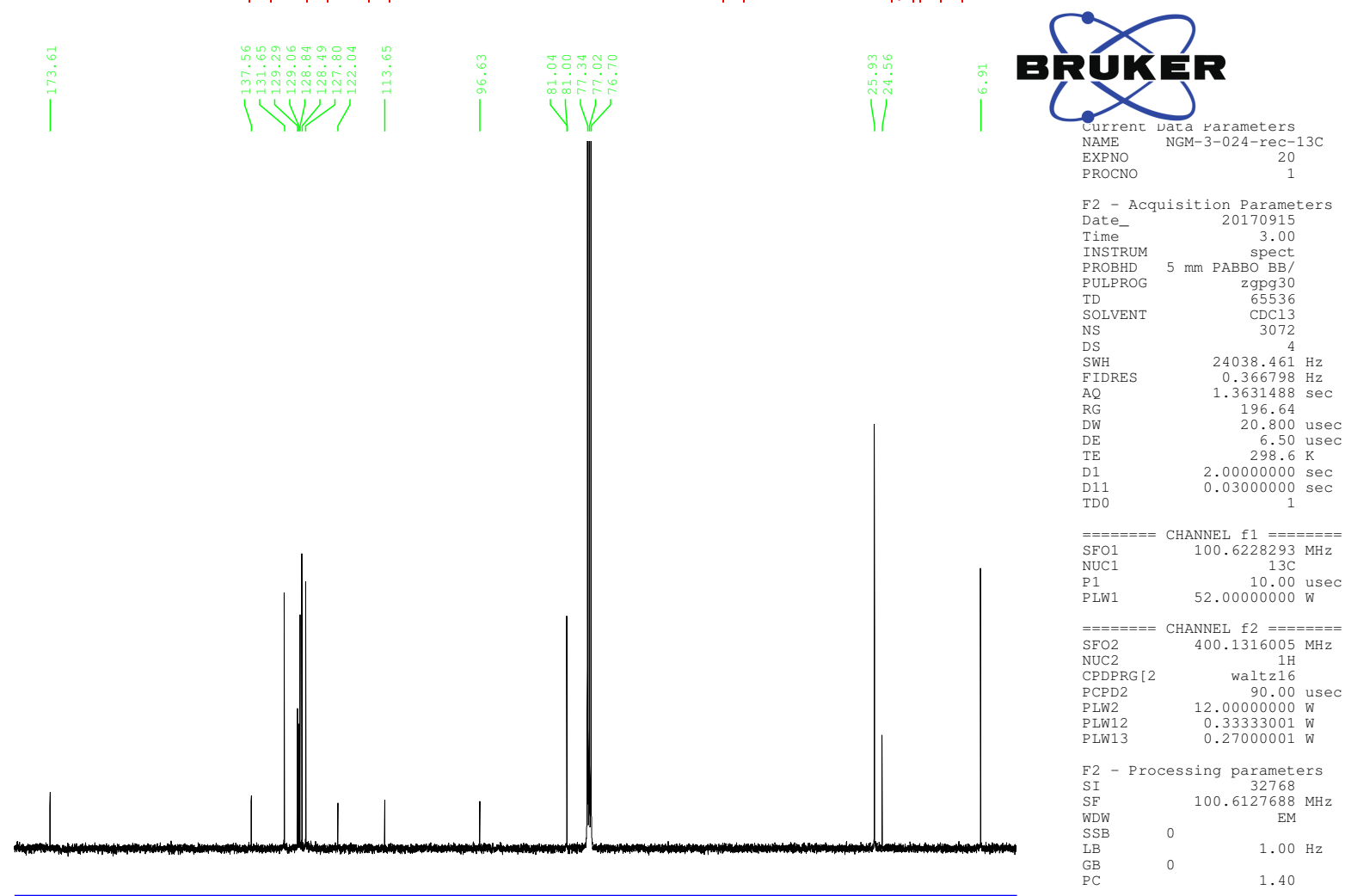

$\begin{array}{lllllllllllllllll}170 & 160 & 150 & 140 & 130 & 120 & 110 & 100 & 90 & 80 & 70 & 60 & 50 & 40 & 30 & 20 & \mathrm{ppm}\end{array}$ 
(E)-N-(tert-butyl)-4-phenyl-2-(phenyl(4,4,5,5-tetramethyl-1,3,2-dioxaborolan-2-yl)methylene)but-3-ynamide (3g)
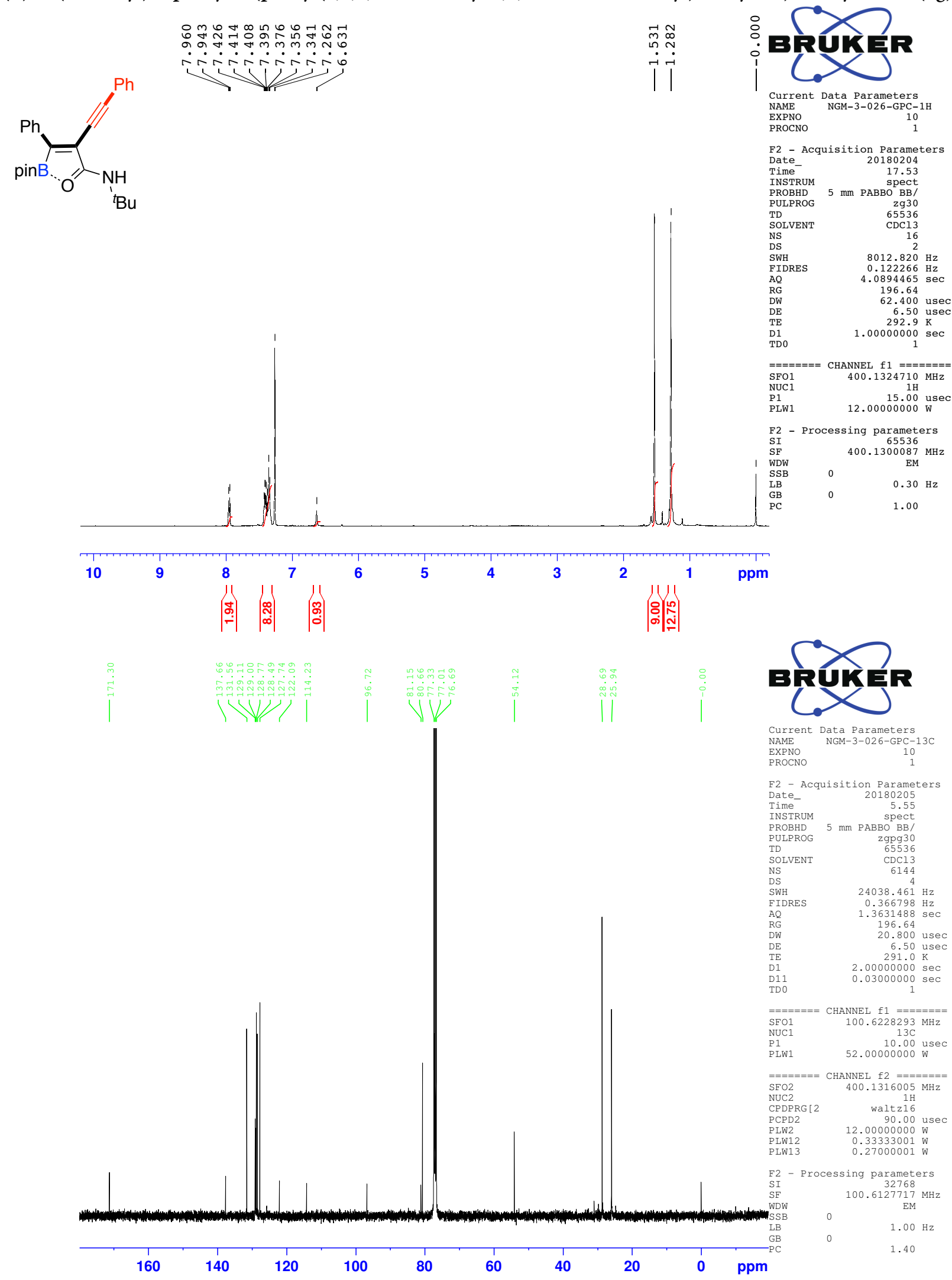
(E)-4-phenyl-2-(phenyl(4,4,5,5-tetramethyl-1,3,2-dioxaborolan-2-yl)methylene)- $\mathrm{N}$-(2-((triisopropylsilyl)oxy)ethyl)but-3ynamide (3i)

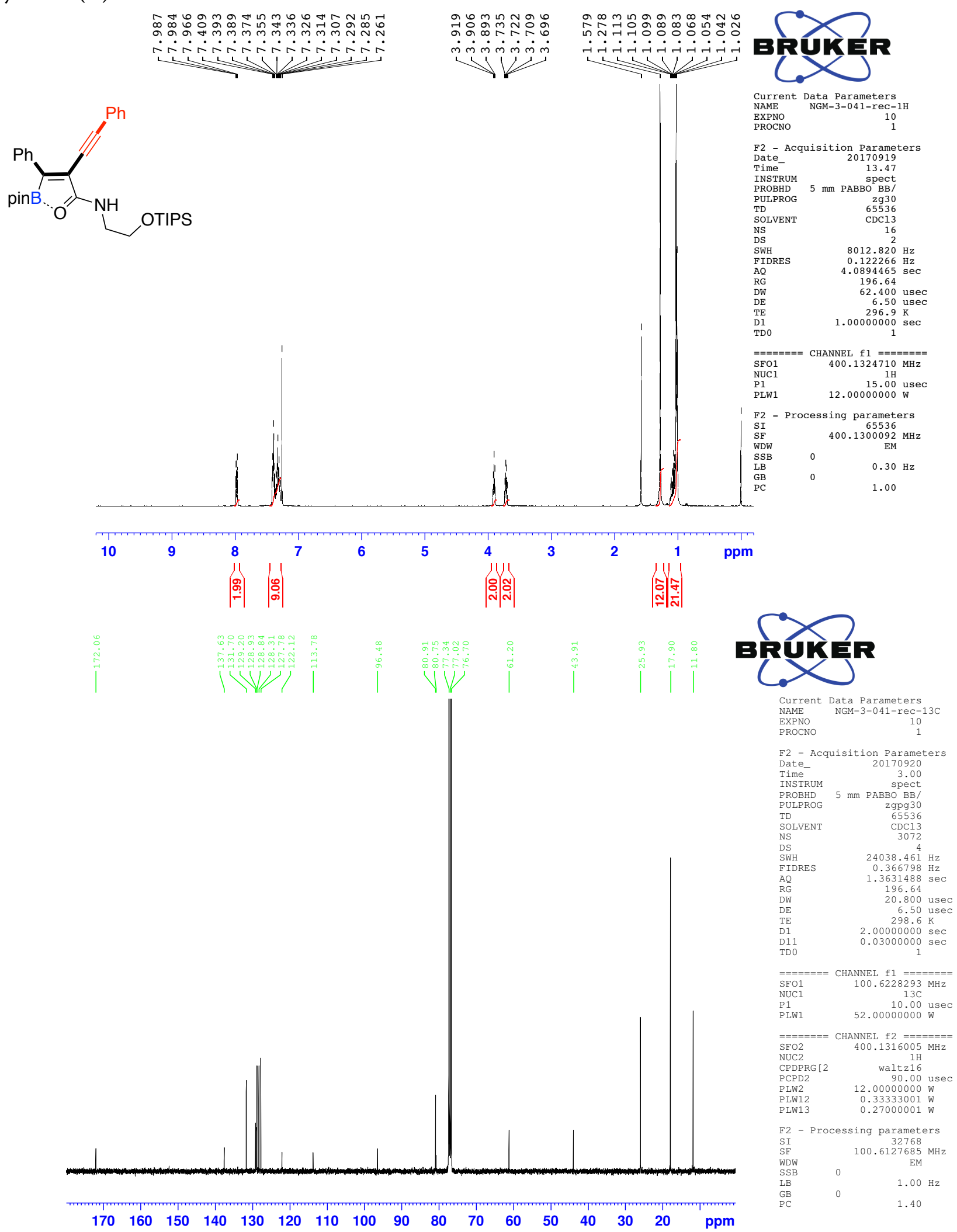


(E)-N-allyl-4-phenyl-2-(phenyl(4,4,5,5-tetramethyl-1,3,2-dioxaborolan-2-yl)methylene)but-3-ynamide (3j)

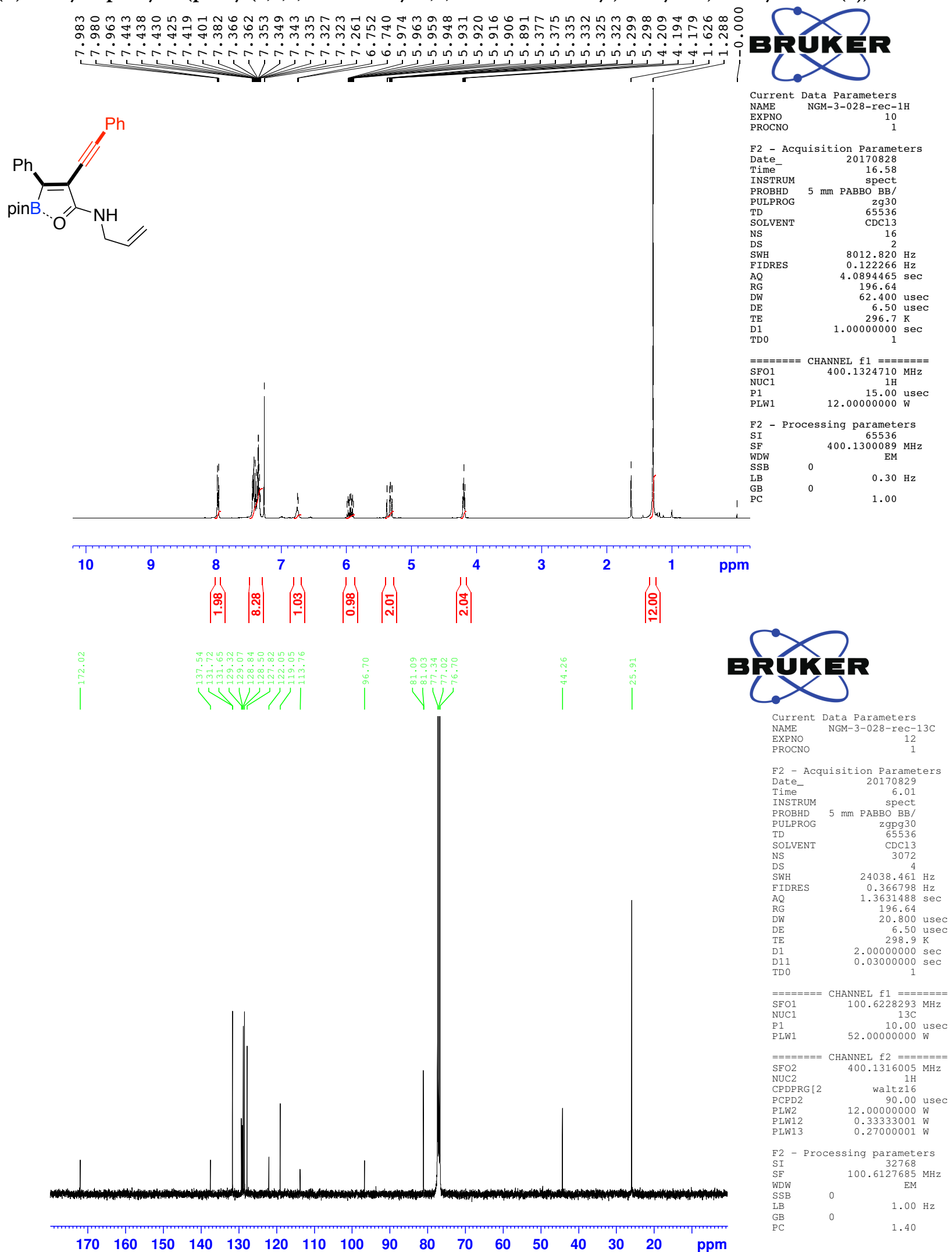


(E)-4-phenyl-2-(phenyl(4,4,5,5-tetramethyl-1,3,2-dioxaborolan-2-yl)methylene)- $N$-(prop-2-yn-1-yl)but-3-ynamide (3k)

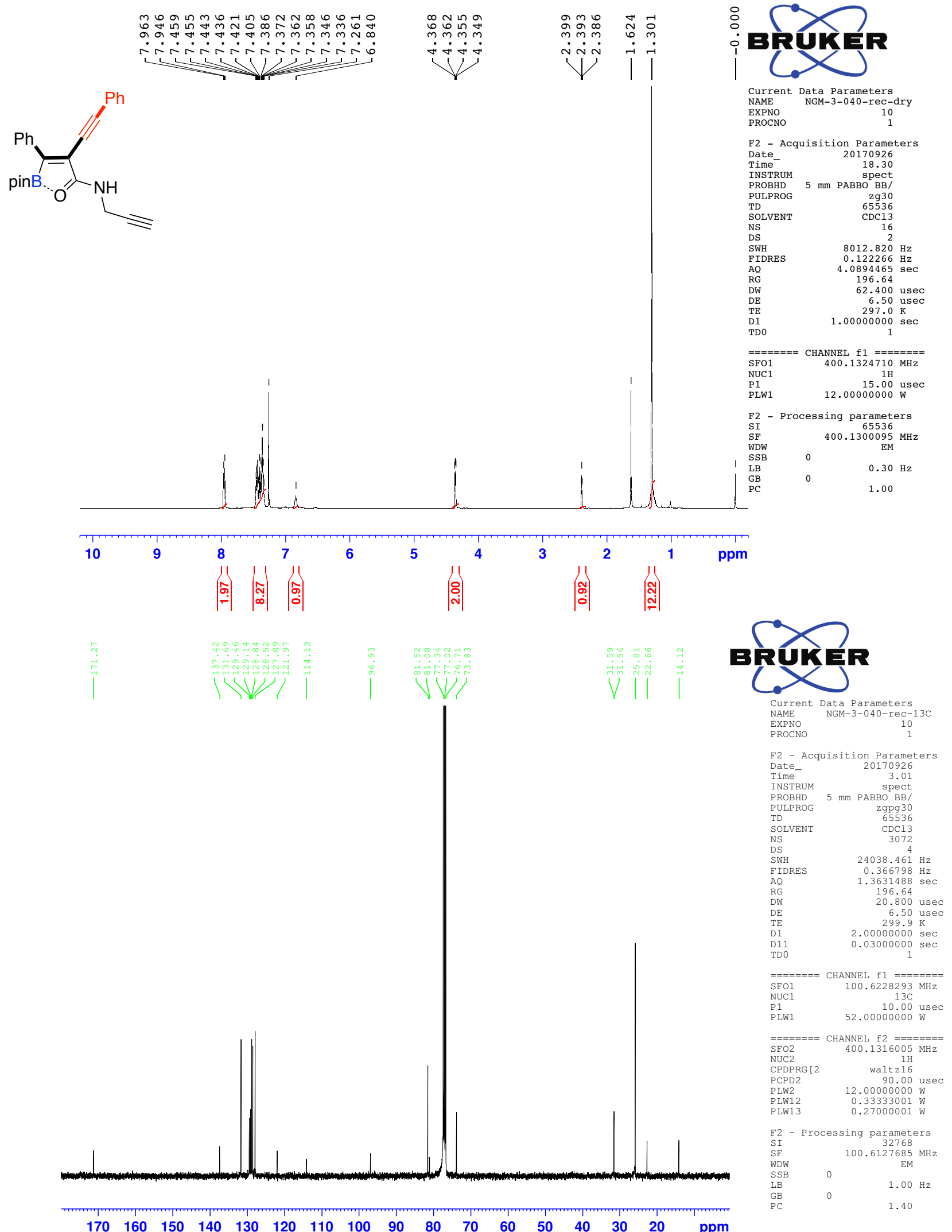

\section{S-43}


(R,E)-4-phenyl-2-(phenyl(4,4,5,5-tetramethyl-1,3,2-dioxaborolan-2-yl)methylene)- $N$-(1-phenylethyl)but-3-ynamide (31)

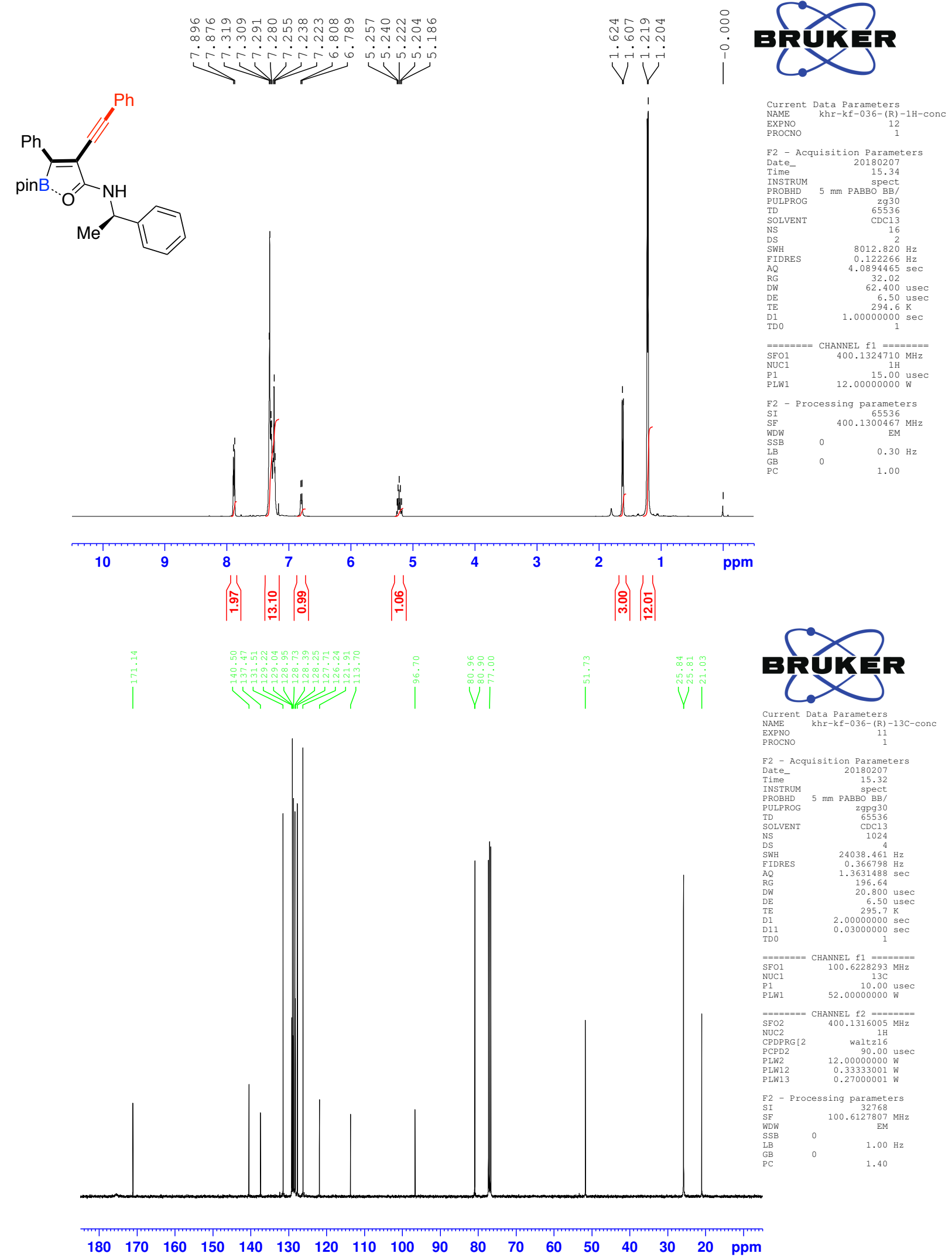

S-44 
(E)-N,4-diphenyl-2-(phenyl(4,4,5,5-tetramethyl-1,3,2-dioxaborolan-2-yl)methylene)but-3-ynamide (3n)
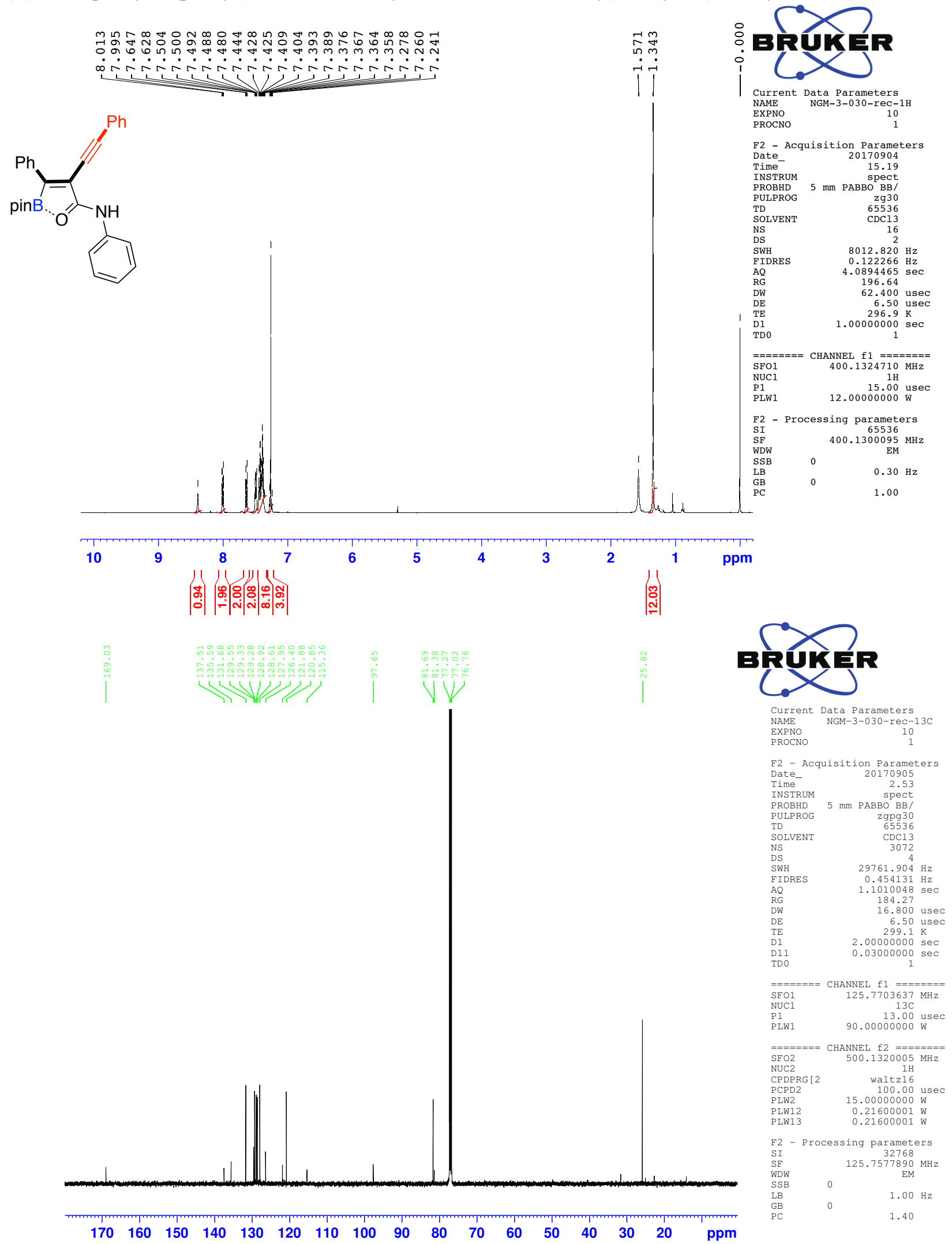
(E)-N-(4-methoxyphenyl)-4-phenyl-2-(phenyl(4,4,5,5-tetramethyl-1,3,2-dioxaborolan-2-yl)methylene)but-3-ynamide (3o)

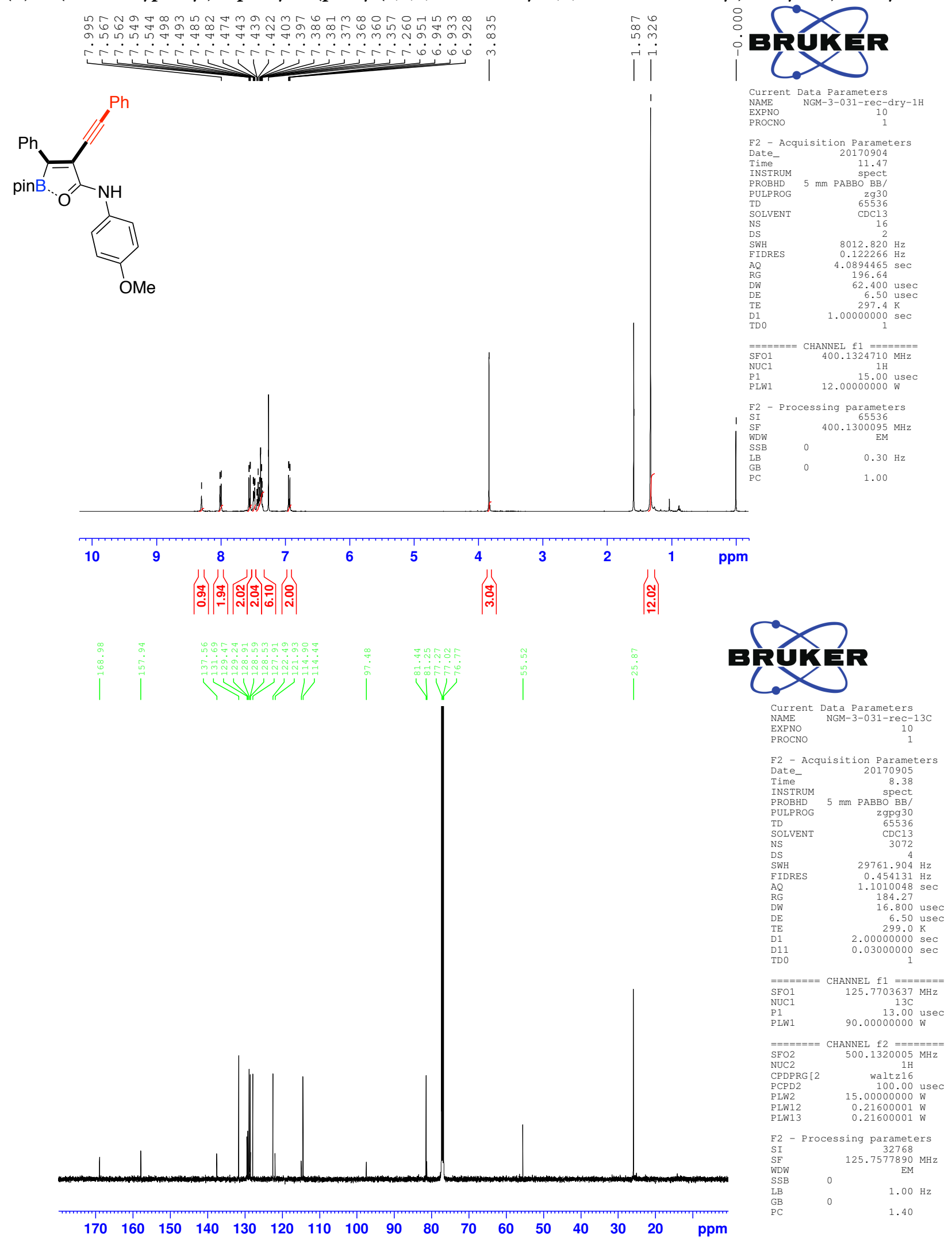


(E)-4-phenyl-2-(phenyl (4,4,5,5-tetramethyl-1,3,2-dioxaborolan-2-yl)methylene)-N-(4-(trifluoromethyl)phenyl)but-3-yna mide (3p)

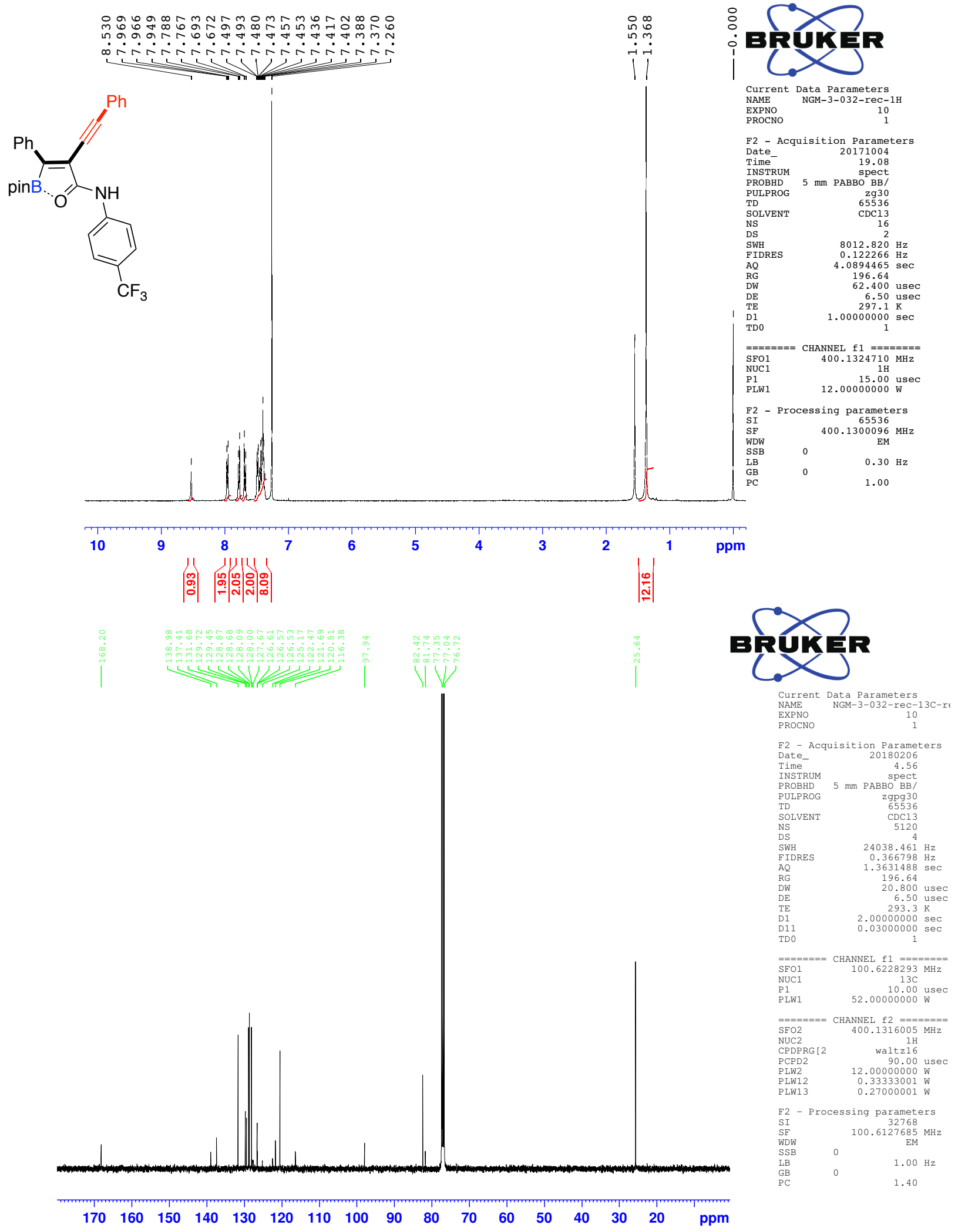


(E)-N-mesityl-4-phenyl-2-(phenyl(4,4,5,5-tetramethyl-1,3,2-dioxaborolan-2-yl)methylene)but-3-ynamide (3q)

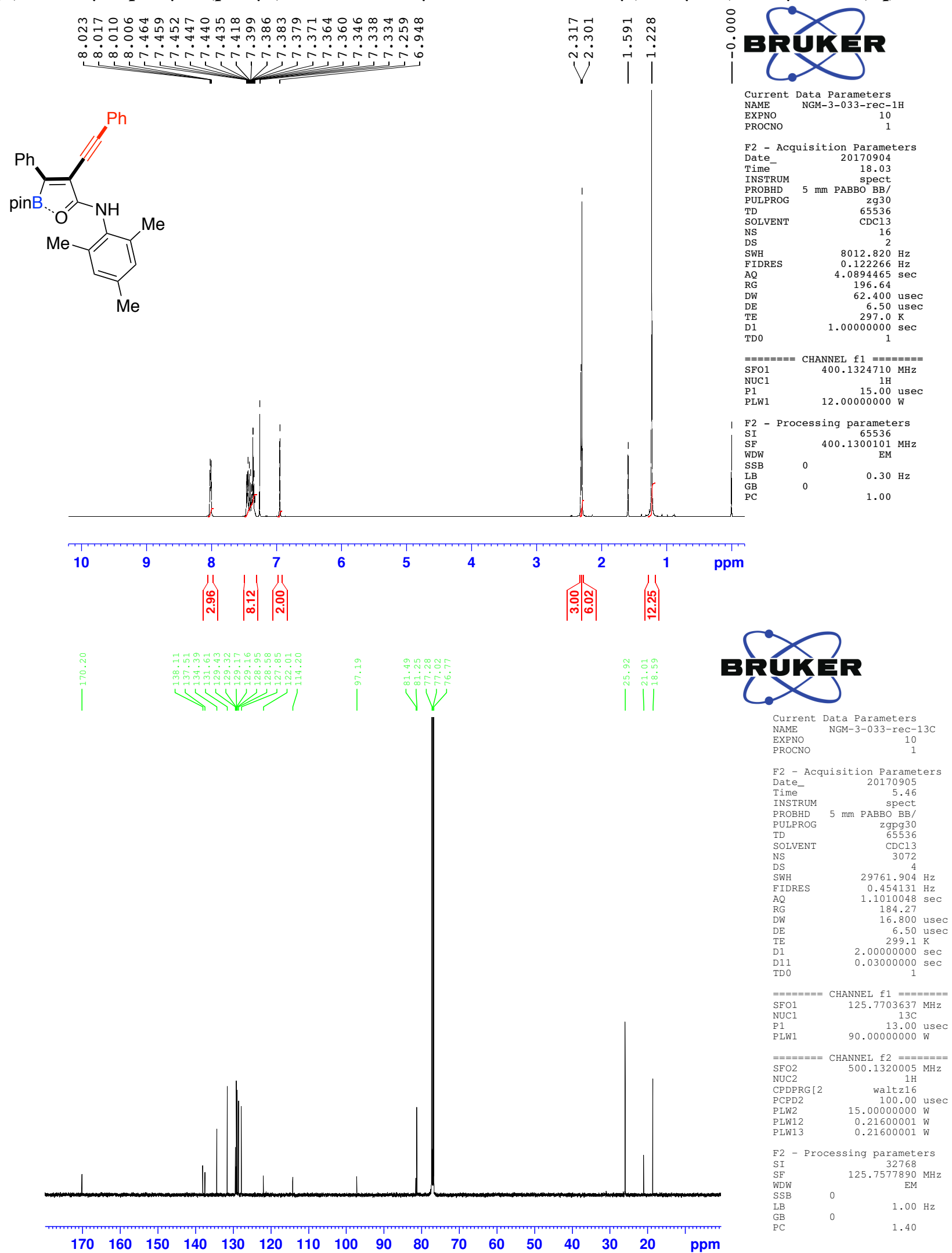


(E)-N-ethyl-2-(naphthalen-1-yl(4,4,5,5-tetramethyl-1,3,2-dioxaborolan-2-yl)methylene)-4-phenylbut-3-ynamide (5a)
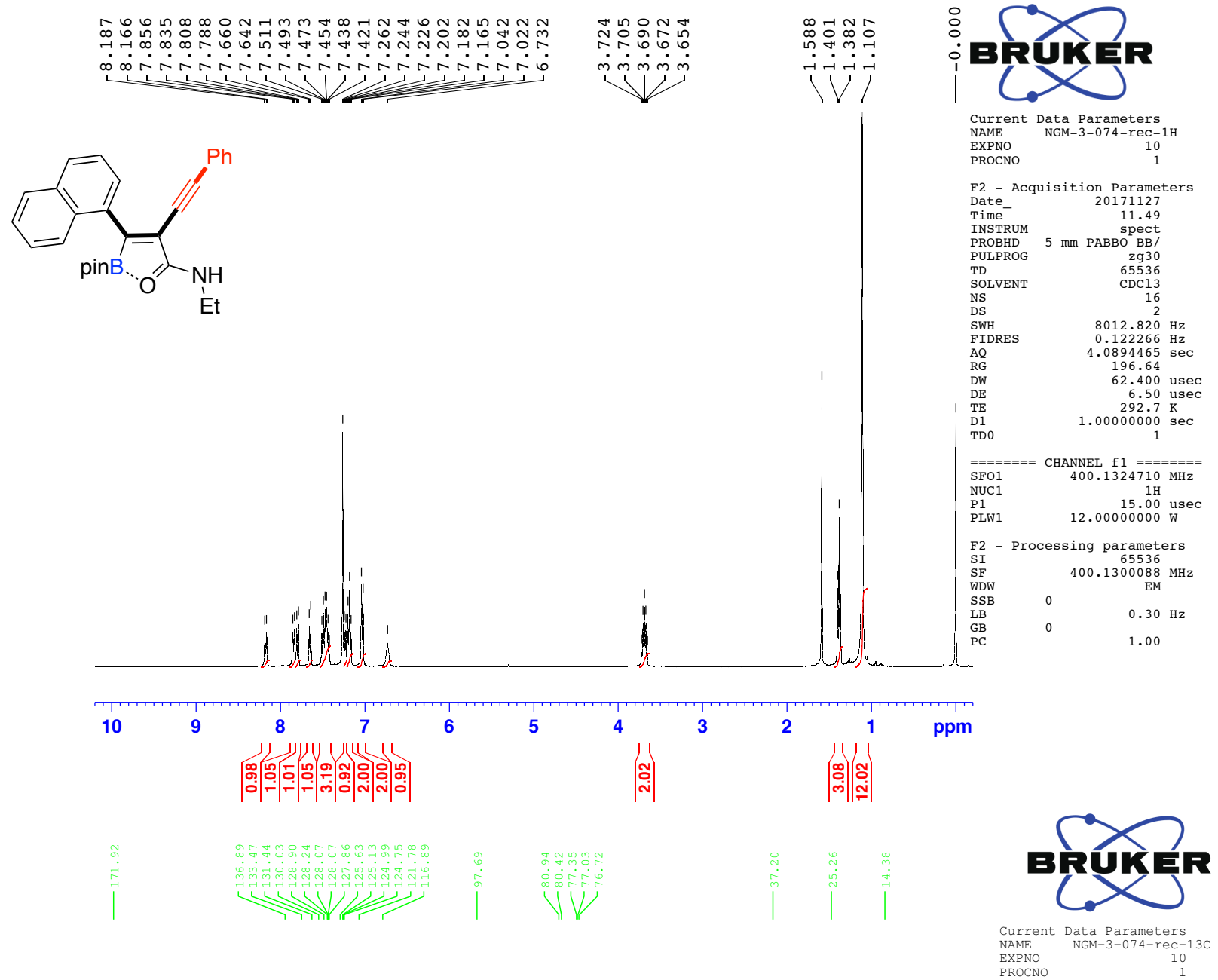

F2 - Acquisition Parameters

F2 - Acquisition Paramet
Date_ 20171128

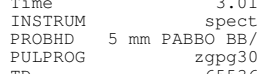

TD

SOLV
NS

NS
DS
SWH
FIDRES

$\begin{array}{lr}24038.461 \mathrm{~Hz} \\ \text { SWH } & 0.366798 \mathrm{~Hz}\end{array}$

$\begin{array}{lr}0.366798 \mathrm{~Hz} \\ \mathrm{AQ} & 1.3631488 \mathrm{sec}\end{array}$

$\begin{array}{lr}196.64 \\ \text { RW } & 20.800 \text { usec }\end{array}$

$\begin{array}{lr}\text { DE } & 6.50 \text { usec } \\ \text { TE } & 294.6 \mathrm{~K} \\ \text { D1 } & 2.00000000 \mathrm{sec}\end{array}$

D11 $\quad \begin{array}{ll}2.00000000 \mathrm{sec} \\ \text { DD0 }\end{array}$
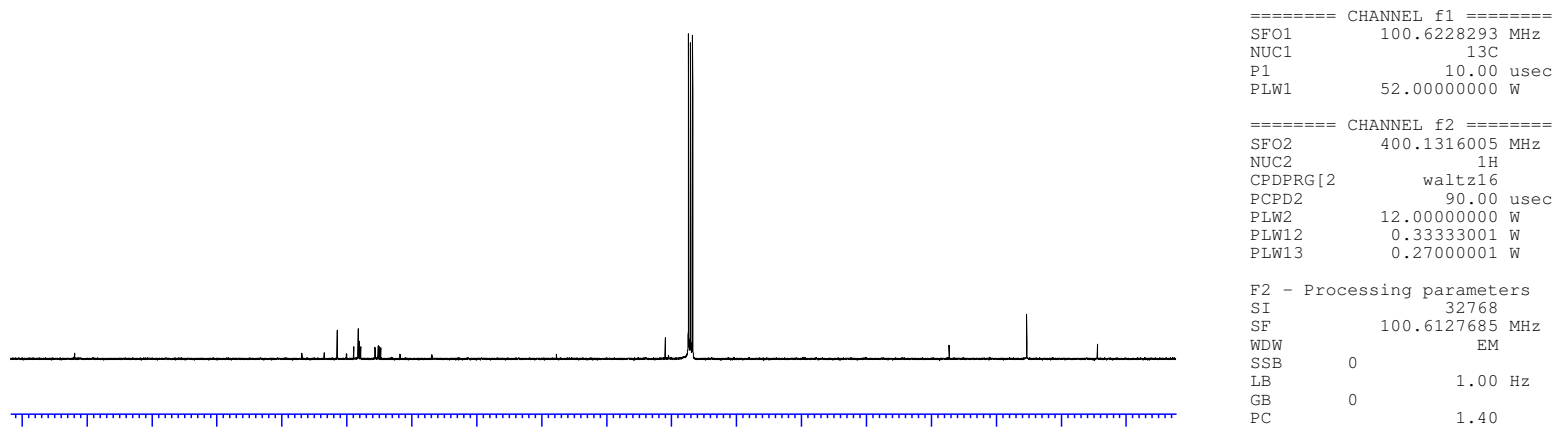

$\begin{array}{lllllllllllllllll}170 & 160 & 150 & 140 & 130 & 120 & 110 & 100 & 90 & 80 & 70 & 60 & 50 & 40 & 30 & 20 & \mathrm{ppm}\end{array}$ 
(E)-N-ethyl-2-(naphthalen-2-yl(4,4,5,5-tetramethyl-1,3,2-dioxaborolan-2-yl)methylene)-4-phenylbut-3-ynamide (5b)

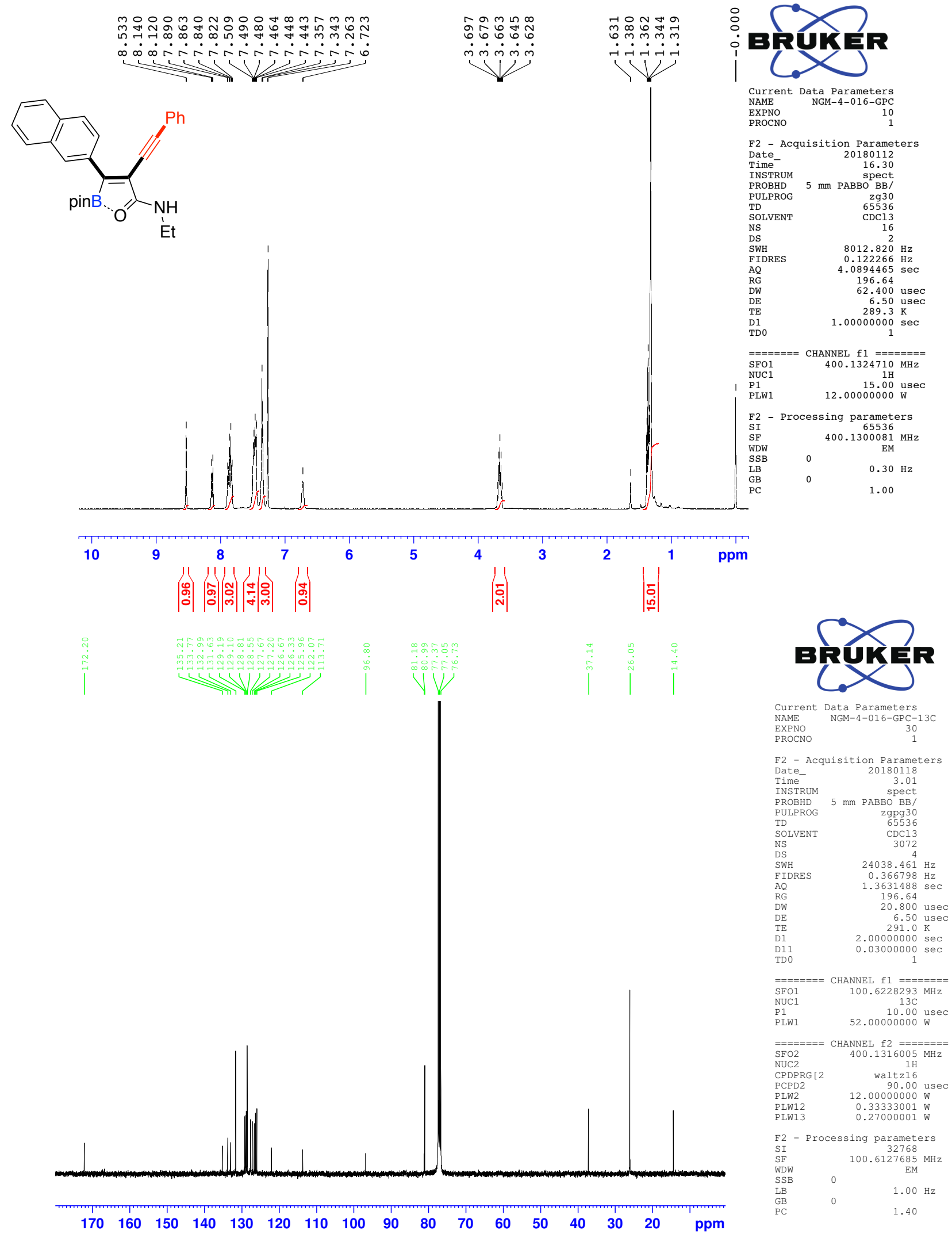


(E)-2-(benzo[d][1,3] dioxol-5-yl(4,4,5,5-tetramethyl-1,3,2-dioxaborolan-2-yl)methylene)-N-ethyl-4-phenylbut-3-ynamide (5c)
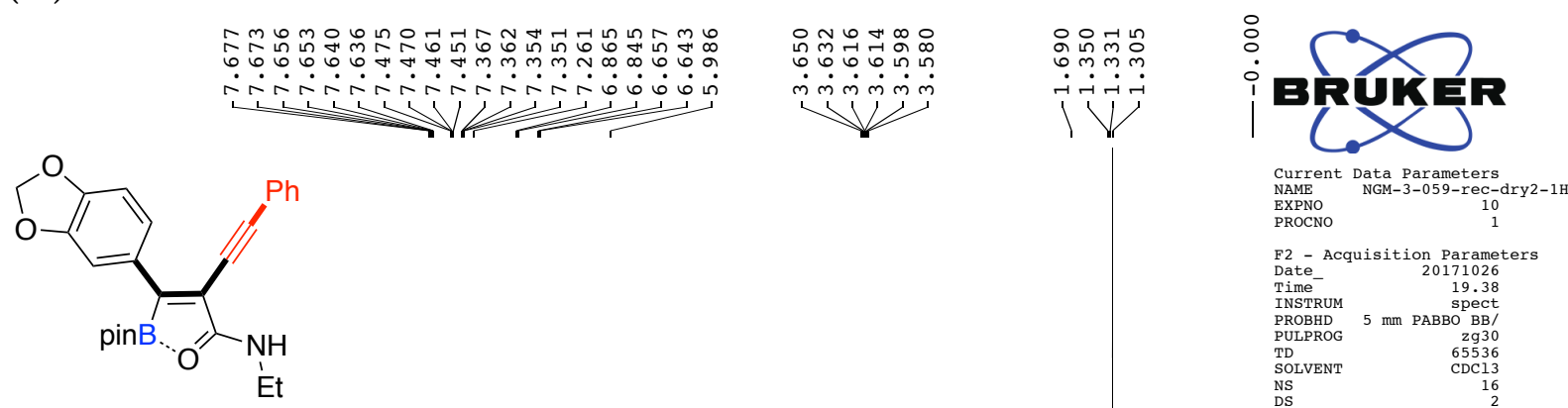
NAME
EXPNO
PROCNO

F2 - Acquisition Parameters 20171026
Time spect
INSTRUM
PROBHD $5 \mathrm{~mm}$ PABBO BB/ PULPROG
P mm PABBO
$\mathrm{zg} 30$ TD

SOLVENT

DS

SW
FIDRES

AQ

RG
DW
DE
TE
$D 1$

D1
TD0

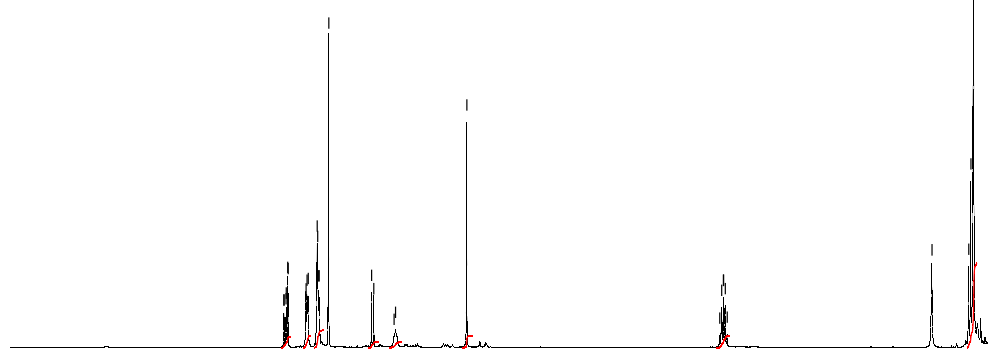

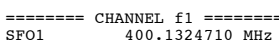

NFO1 $400.13241 \mathrm{H}$

$\begin{array}{lr}\text { P1 } & 15.00 \text { usec } \\ \text { PLW1 } & 12.00000000 \text { W }\end{array}$

$\begin{array}{lc}\text { F2 } & \text { - Processing parameters } \\ \text { SI } & 65536 \\ \text { SF } & 400.1300089 \mathrm{MHz} \\ \text { WDW } & \text { EM }\end{array}$

$\mathrm{SSB}$
$\mathrm{LB}$

PC

$0.30 \mathrm{~Hz}$
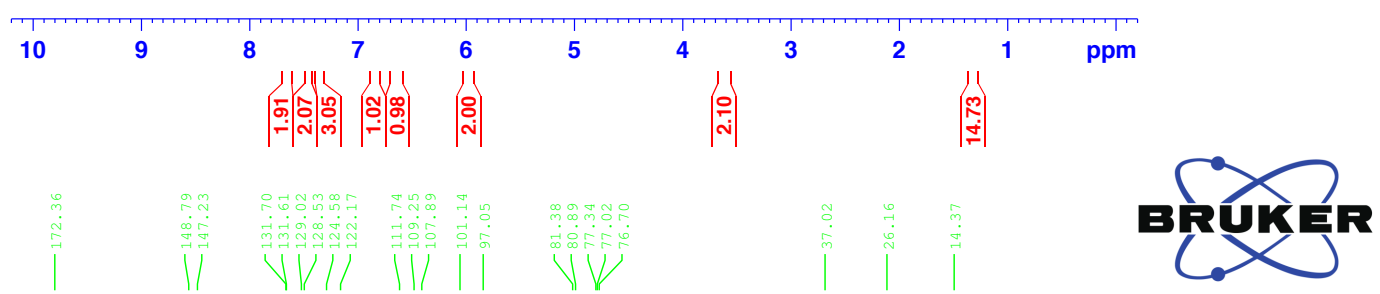

Current Data Parameters
NAME NGM-3-059-rec-dry2-13C NXMENO
PROCNO

F2 - Acquisition Parameters

Date

Time-

spect
PROBHD $5 \mathrm{~mm}$ PABBO BB/

SOLVENT

NS
DS
SWH

DS
SWH
FIDRES
AQ
RG

DW
DE
TE
D1
D11
TD0

$1.3631488 \mathrm{sec}$
196.64

196.64 used
20.800
6.50

$298.8 \mathrm{~K}$

$2.00000000 \mathrm{sec}$
$0.03000000 \mathrm{sec}$

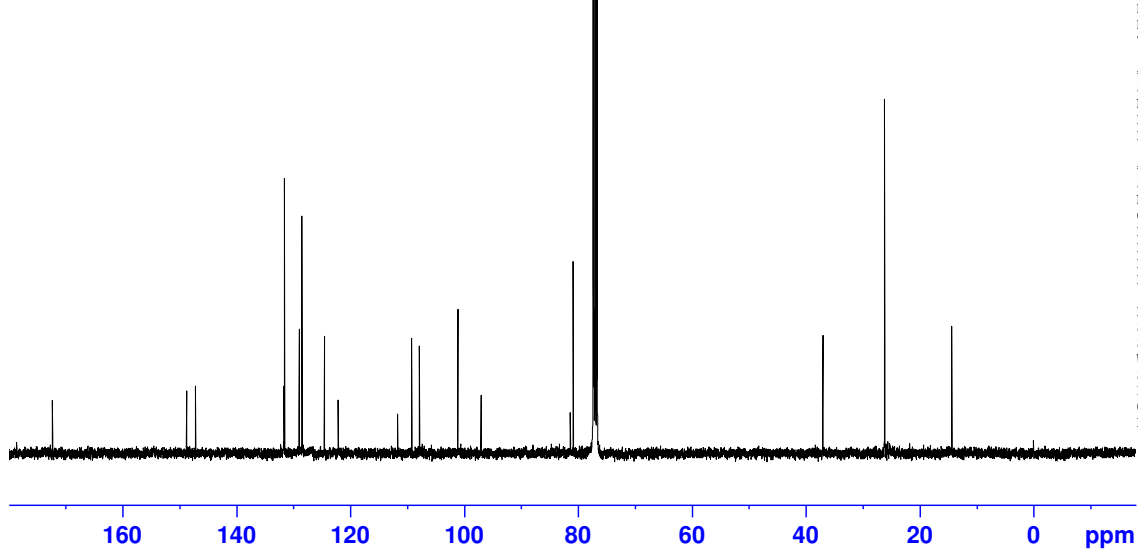

$======8$ CHANNEL $\mathrm{f} 1 \mathrm{l}=======$
FO1 $100.6228293 \mathrm{MHz}$
WC1

$\begin{array}{ll}13 \mathrm{C} \\ 1 & 10.00 \mathrm{usec}\end{array}$

$== \pm== \pm=$ CHANNEL $f 2== \pm== \pm=$
SFO2 $400.1316005 \mathrm{MHz}$

$\begin{array}{lr}\text { SFO2 } & 400.1316005 \\ \text { NUC2 } & 1 \mathrm{H} \\ \text { CPDPRG I2 } & \text { waltz16 }\end{array}$

90.00 us

$\begin{array}{lr}\text { PLW2 } & 12.00000000 \mathrm{~W} \\ \text { PLW12 } & 0.33333001 \mathrm{~W} \\ \text { PLW13 } & 0.27000001 \mathrm{~W}\end{array}$

F2 - Processing parameters

$\begin{array}{ll}\text { SI } & 32768 \mathrm{MHz} \\ \text { SF } & 100.6127685 \mathrm{MHz}\end{array}$

$1.00 \mathrm{~Hz}$ 
(E)-N-ethyl-2-((4-methoxyphenyl)(4,4,5,5-tetramethyl-1,3,2-dioxaborolan-2-yl)methylene)-4-phenylbut-3-ynamide (5d)

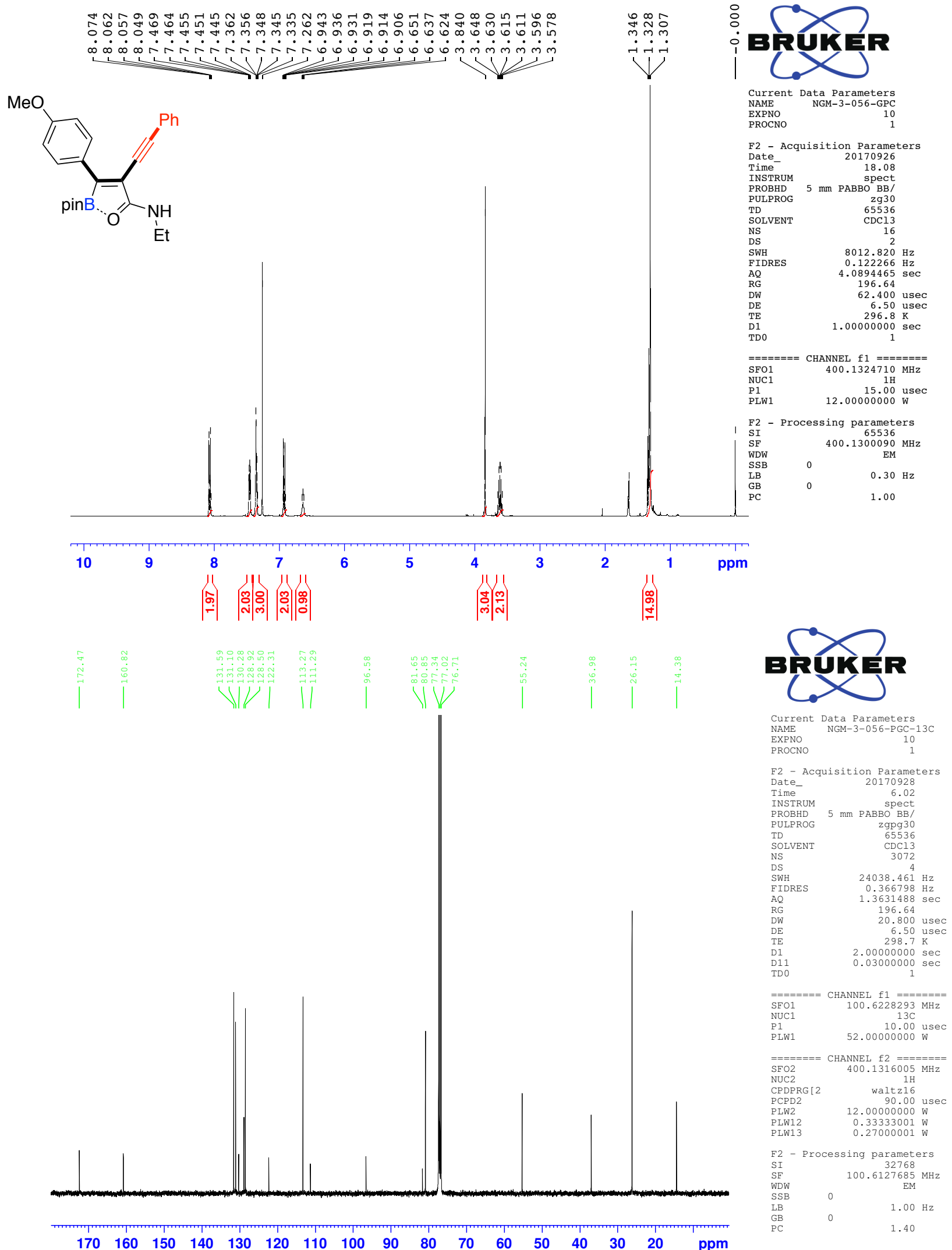

S-52 
(E)-N-ethyl-2-((4-(methylthio)phenyl)(4,4,5,5-tetramethyl-1,3,2-dioxaborolan-2-yl)methylene)-4-phenylbut-3-ynamide (5e)
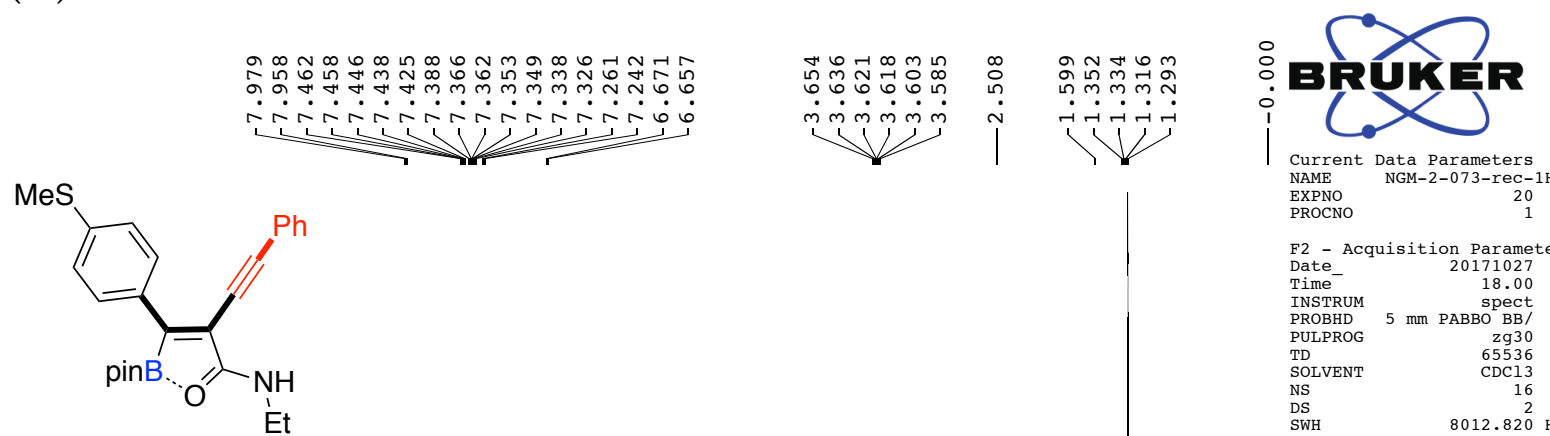

F2 - Acquisition Parameters

\begin{tabular}{cr} 
Date & Acquisition \\
Time & 20171027 \\
\hline
\end{tabular}

spect
INSTRUM

PULPROG $5 \mathrm{~mm}$

SOLVENT

NS

SWH $\quad 8012.820 \mathrm{~Hz}$

$\begin{array}{lr}\text { FIDRES } & 0.122266 \mathrm{~Hz} \\ \text { AQ } & 4.0894465 \mathrm{sec}\end{array}$

$\begin{array}{ll}\text { RG } & 196.64 \\ \text { DW } & 62.400 \text { usec }\end{array}$

$\begin{array}{lr}\mathrm{DE} & 6.50 \text { us } \\ \mathrm{TE} & 297.1 \mathrm{~K}\end{array}$

$\begin{array}{ll}\text { TE } & 1.00000000 \mathrm{~K} \\ \text { D1 } & 1\end{array}$

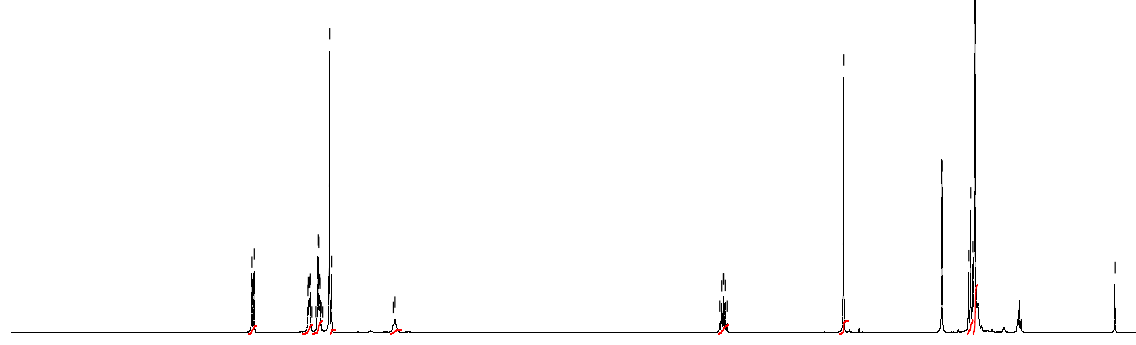

$=======\quad$ CHANNEL $\mathrm{f} 1 \quad========$
SFO1 $\quad 400.1324710 \mathrm{MHz}$

NUC1 $11 \mathrm{H}$

$\begin{array}{lr}\text { P1 } & 15.00 \text { usec } \\ \text { PLW1 } & 12.00000000 \text { W }\end{array}$

F2 - Processing parameter

$\begin{array}{lr}\text { F2 - Processing parameters } \\ \text { SI } & 65536 \\ \text { SF } & 400.1300092 \mathrm{MHz}\end{array}$

$\begin{array}{ll}\text { WDW } \\ \text { SSB } & 0\end{array}$

$\begin{array}{lll}\mathrm{SSB} & 0 & 0.30 \mathrm{~Hz} \\ \mathrm{LB} & 0 & 1.00\end{array}$
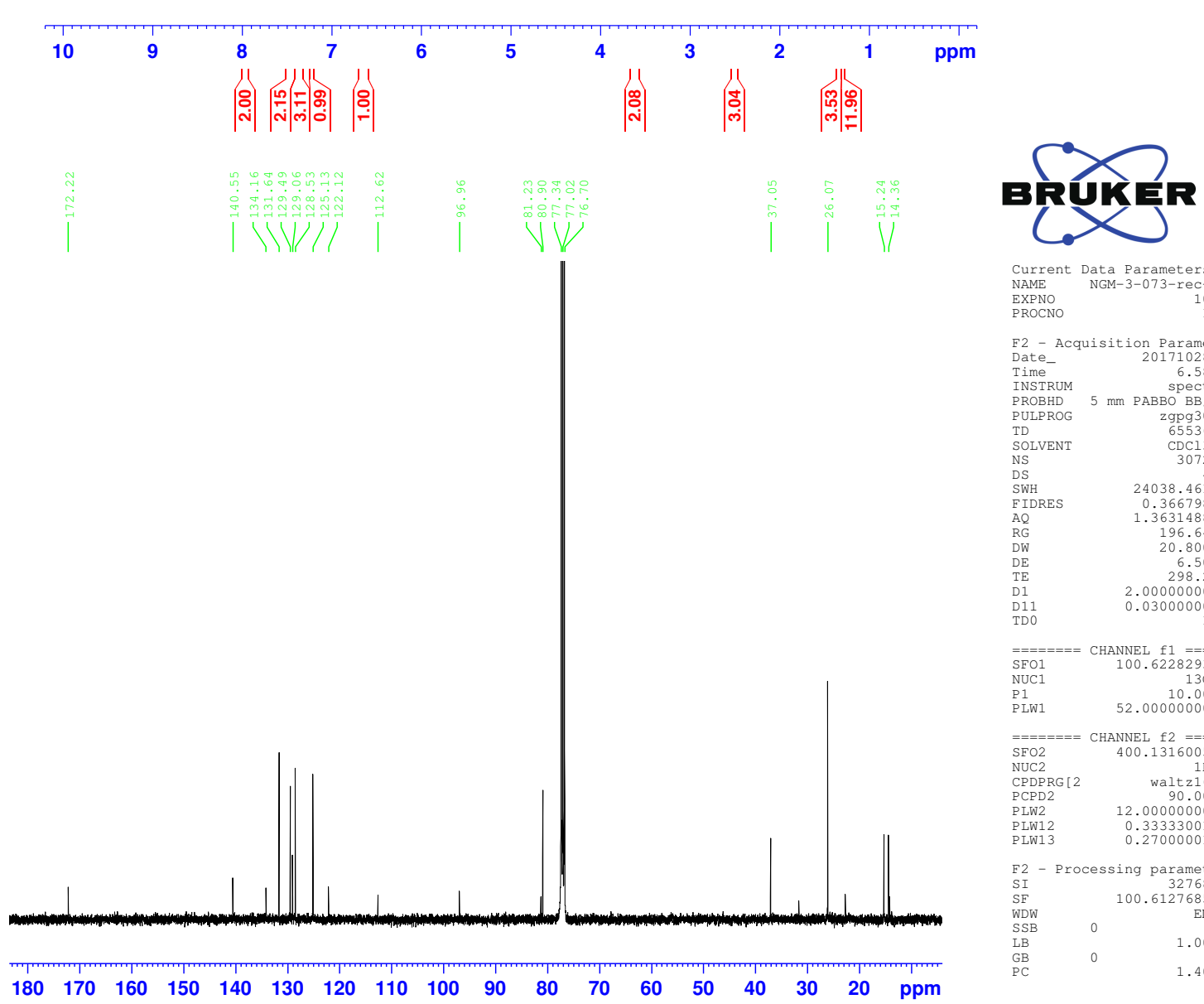

$\begin{array}{ll}\text { Current } & \text { Data Parameters } \\ \text { NAME } & \text { NGM-3-073-rec-13C }\end{array}$

EXPNO

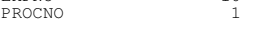

F2 - Acquisition Parameters

F2 - Acquisition Parame
Date_
Time

INSTRUM spec

PROBHD
PULPROG

NS

$\begin{array}{lr}\text { DS } & 4 \\ \text { SWH } & 24038.461 \mathrm{~Hz} \\ \text { FIDRES } & 0.366798 \mathrm{~Hz}\end{array}$

AQ $\quad \begin{array}{ll}0.366798 \mathrm{~Hz} \\ \mathrm{AG}\end{array}$

$\begin{array}{lr}\text { RG } & 196.64 \\ \text { DW } & 20.800 \text { usec } \\ \text { DE } & 6.50 \text { usec } \\ \text { TE } & 298.2 \text { K }\end{array}$

$\begin{array}{ll}\text { TE } & 2.00000000 \mathrm{~K} \\ \text { D1 } & 29.5\end{array}$

D11 $\quad 0.03000000 \mathrm{sec}$

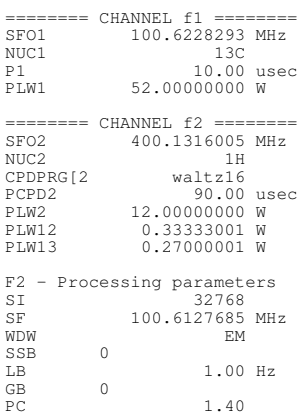


(E)-2-((4-(diphenylamino)phenyl)(4,4,5,5-tetramethyl-1,3,2-dioxaborolan-2-yl)methylene)-N-ethyl-4-phenylbut-3-ynami de $(5 f)$

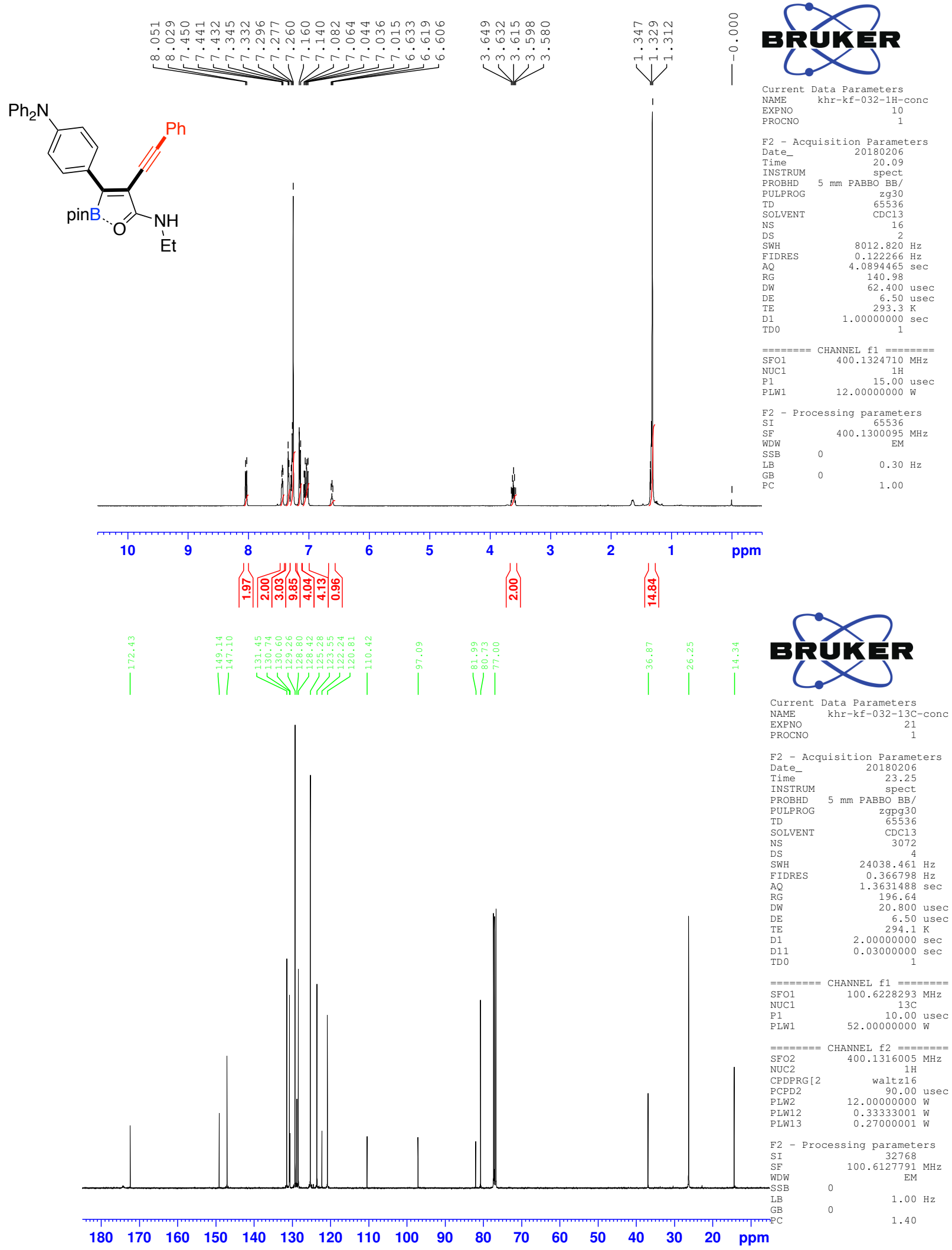

\section{S-54}


(E)-2-((4-(9H-carbazol-9-yl)phenyl)(4,4,5,5-tetramethyl-1,3,2-dioxaborolan-2-yl)methylene)- $N$-ethyl-4-phenylbut-3-ynam ide $(5 \mathbf{g})$

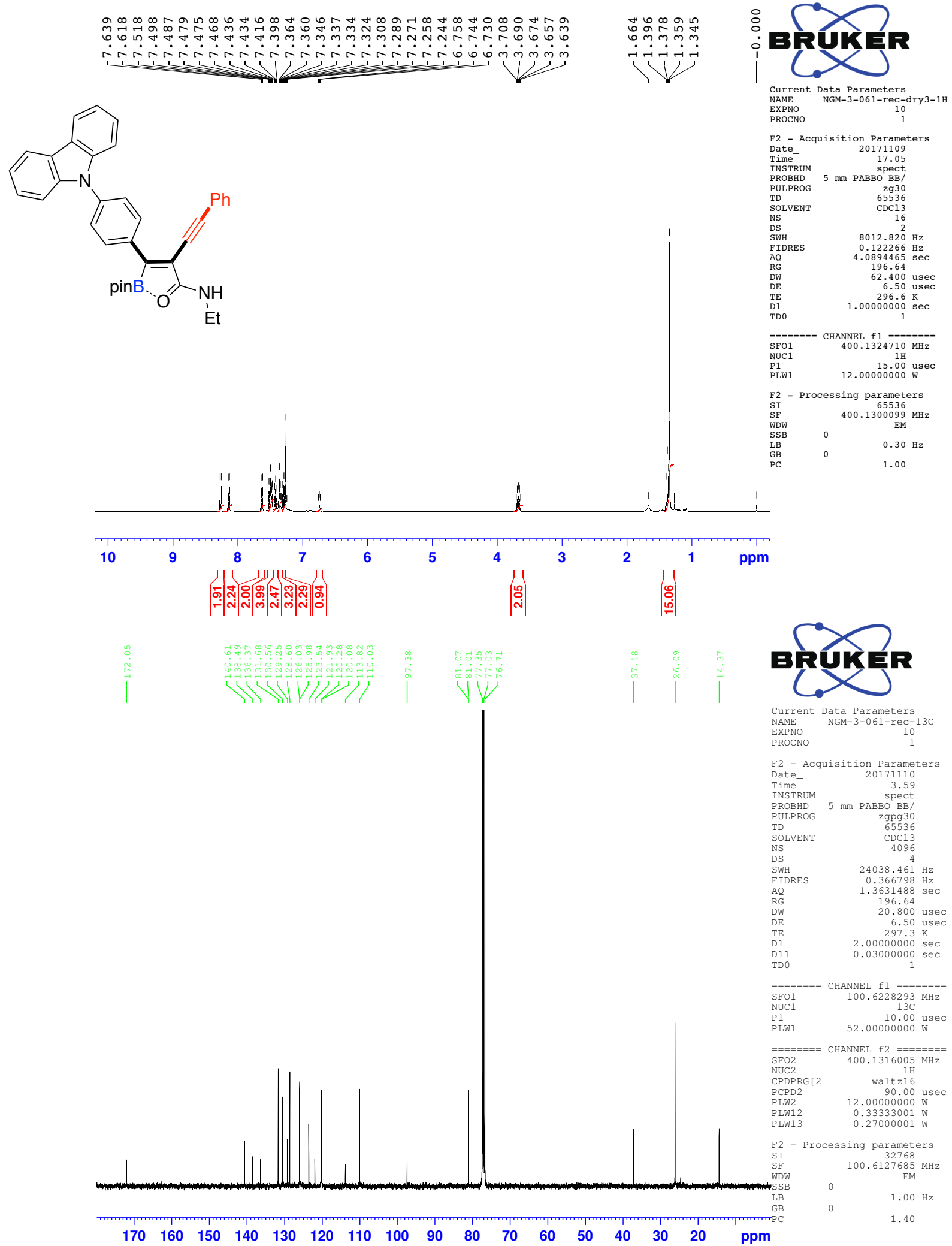

\section{S-55}


(E)-2-((4-chlorophenyl)(4,4,5,5-tetramethyl-1,3,2-dioxaborolan-2-yl)methylene)- $\mathrm{N}$-ethyl-4-phenylbut-3-ynamide (5h)
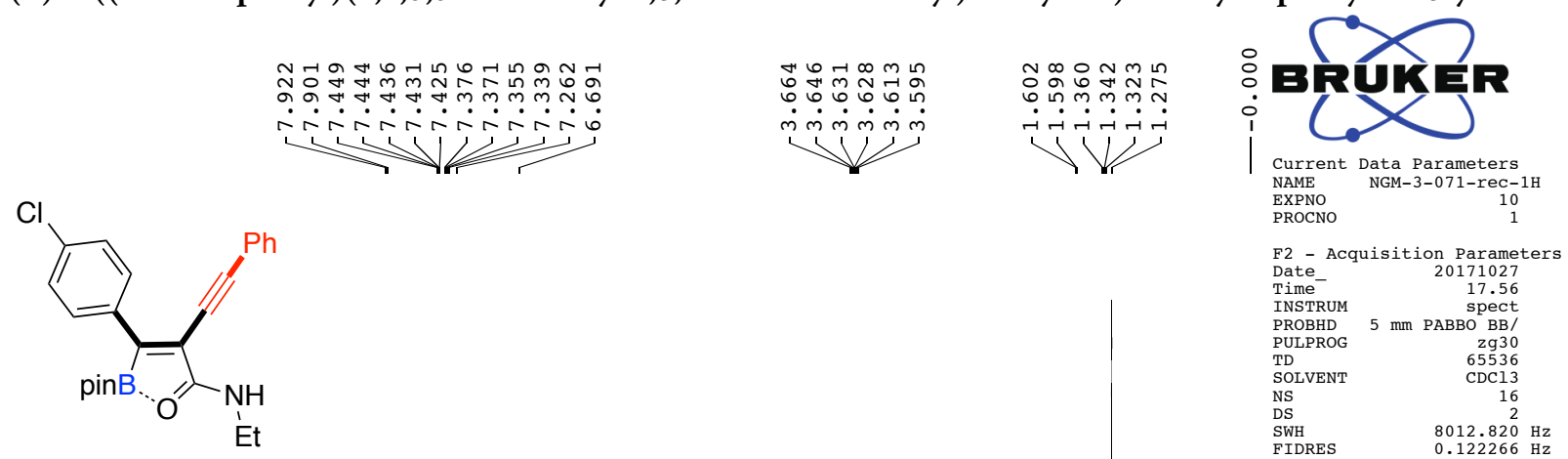

EXPNO
PROCNO

F2 - Acquisition Parameters

Date_ 20171027

Time-
INSTRU

spect
PROBHD $5 \mathrm{~mm}$ PABBO BB/

PULPROG

PULPROG
TD

SOLVENT

NS
DS
SWH

SWH $\quad 8012.820 \mathrm{~Hz}$

$\begin{array}{ll}\text { FIDRES } & 0.122266 \mathrm{~Hz} \\ \text { AQ } & 4.0894465 \mathrm{sec}\end{array}$

RG

$\mathrm{DW}$
$\mathrm{DE}$
$\mathrm{TE}$

62.400 usec
$6.50 \mathrm{usec}$

$\begin{array}{ll}297.1 \mathrm{~K} \\ 1 & 1.00000000 \mathrm{sec}\end{array}$

DO

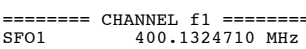

$\begin{array}{lr}\text { SFO1 } & 400.1324710 \mathrm{MHz} \\ \text { NUC1 } & 1 \mathrm{H}\end{array}$

$\begin{array}{lr}\text { P1 } & 15.00 \text { usec } \\ \text { PLW1 } & 12.00000000 \mathrm{~W}\end{array}$
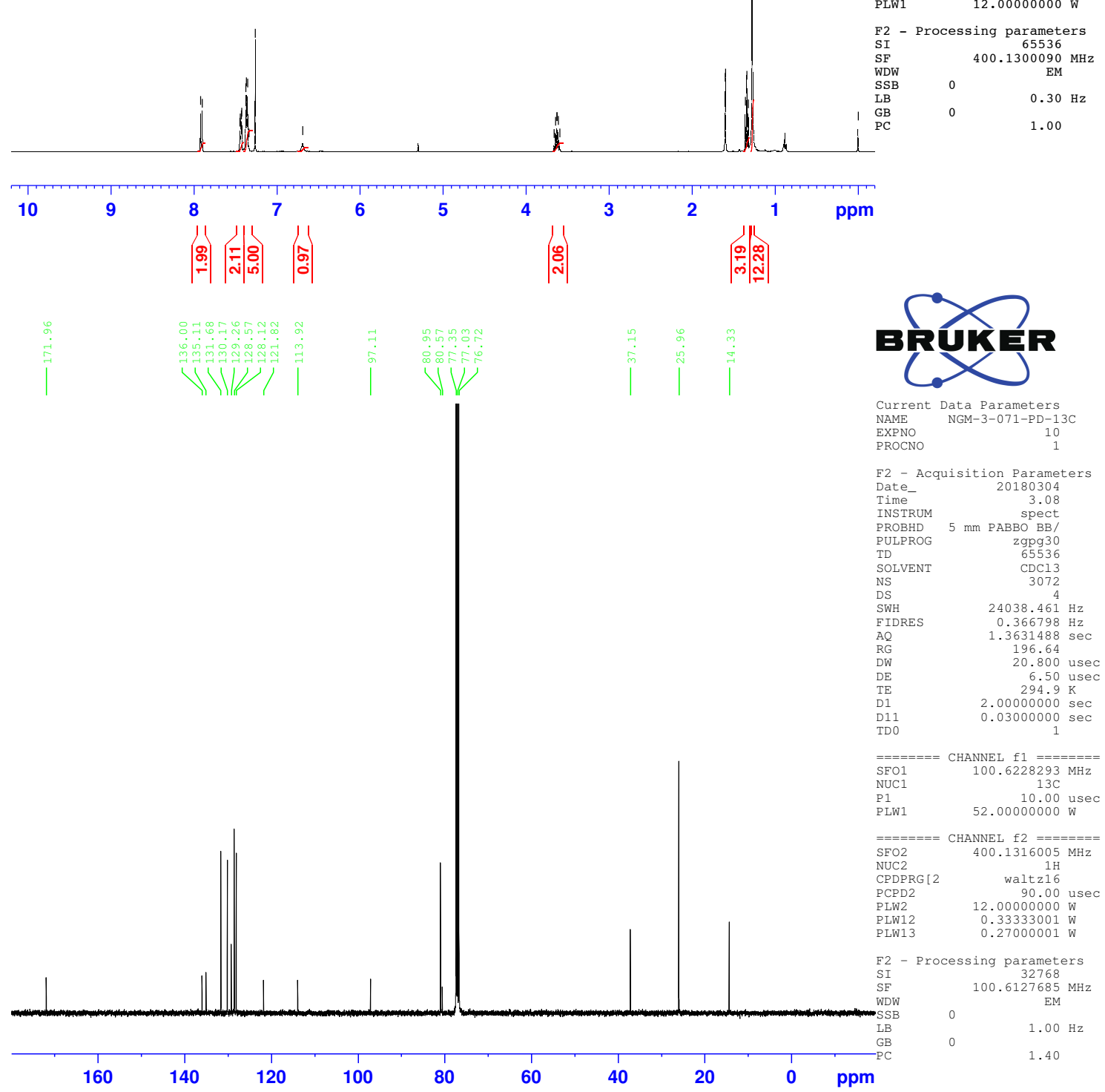

S-56 
(E)-N-ethyl-4-phenyl-2-((4,4,5,5-tetramethyl-1,3,2-dioxaborolan-2-yl)(4-(trifluoromethyl)phenyl)methylene)but-3-ynami de $(\mathbf{5 i})$
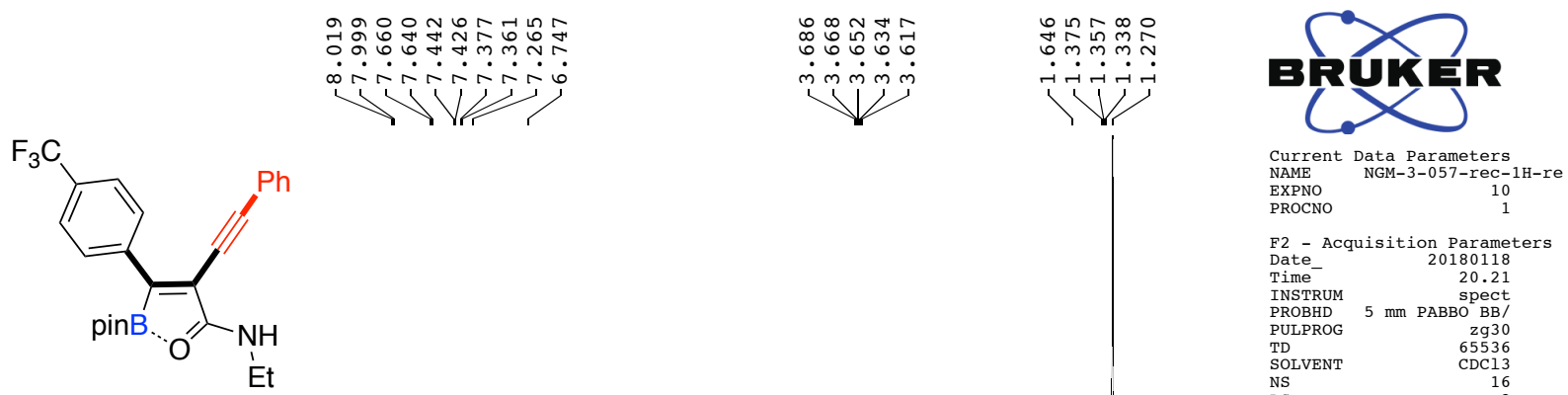

F2 - Acquisition Parameters

\begin{tabular}{lr} 
Date & Acquisition \\
Time & 20180118 \\
\hline
\end{tabular}

INSTRUM spect

PROBHD $5 \mathrm{~mm}$ PABBO BB/

PULPRO
TD

SOLVEN

NS

2

$\begin{array}{ll}\text { SWH } & 8012.820 \mathrm{~Hz} \\ \text { FIDRES } & 0.122266 \mathrm{~Hz}\end{array}$

$\begin{array}{lr}\text { AQ } & 4.0894465 \mathrm{sec} \\ \text { RG } & 196.64 \mathrm{usec} \\ \text { DW } & 62.400 \mathrm{us}\end{array}$

$\begin{array}{lr}\mathrm{DW} & 62.400 \mathrm{usec} \\ \mathrm{DE} & 6.50 \text { usec }\end{array}$

$\begin{array}{lr}\text { TE } & 289.8 \mathrm{~K} \\ \text { D1 } & 1.00000000 \mathrm{sec} \\ \text { TD0 } & 1\end{array}$

$=======$ CHANNEL $f 1 \quad========$
SFO1
$400.1324710 \mathrm{MHz}$

NUC1 $\quad 400.13247 \mathrm{H}$

$\begin{array}{lr}\text { P1 } & 15.00 \text { usec } \\ \text { PLW1 } & 12.00000000 \mathrm{~W}\end{array}$

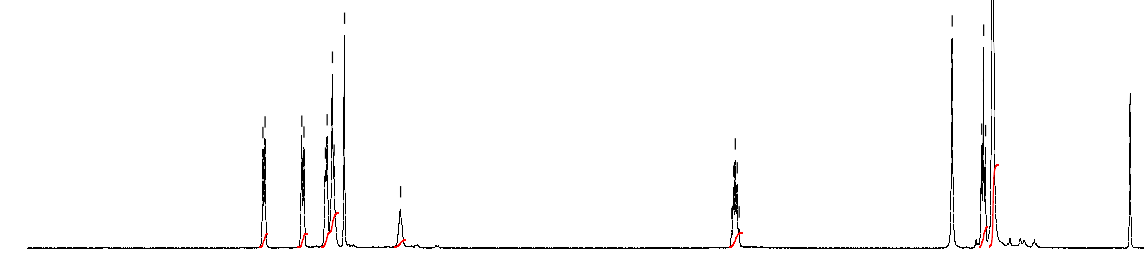

F2 - Processing parameters

$\begin{array}{ll}\mathrm{SI} & 65536 \\ \mathrm{SF} & 400.1300078 \mathrm{MHz}\end{array}$

WDW $\quad 400.1300078 \mathrm{MM}$

$\begin{array}{lll}\text { LB } & 0 & 0.30 \mathrm{~Hz} \\ \text { GB } & 0 & 1.00\end{array}$
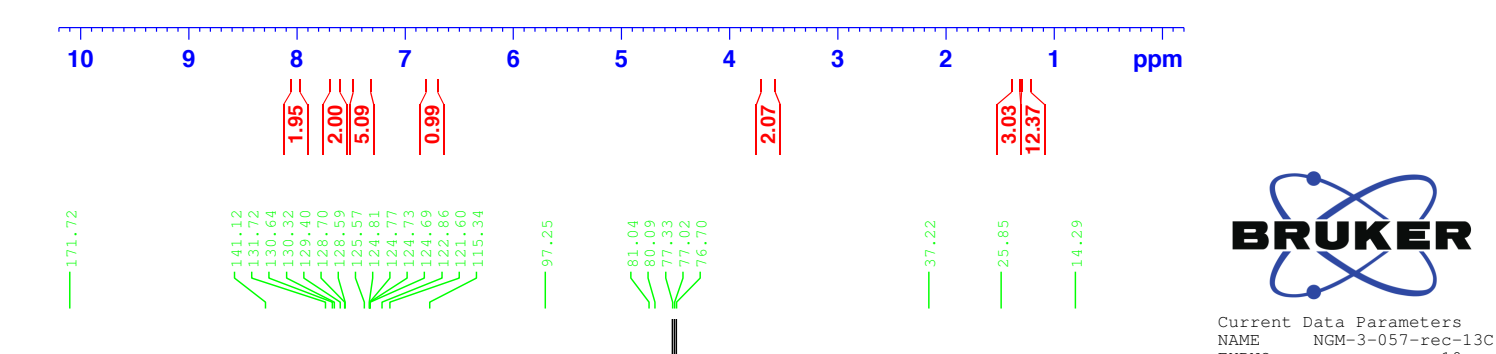
EXPNO

PROCI

F2 - Acquisition Parameters

Date_ $\quad 20180119$

$\begin{array}{ll}\text { Time } & 4.57 \\ \text { INSTRUM } & \text { S.5 }\end{array}$

spect
PROBHD $5 \mathrm{~mm}$ PABBO BB/

zgpg30

TD

SOLVEN
NS
DS
SWH

$\begin{array}{lr}\text { FIDRES } & 0.366798 \mathrm{~Hz} \\ \text { AQ } & 1.3631488 \mathrm{sec}\end{array}$

$\begin{array}{lr}\text { RG } & 1.3651488 \text { sec } \\ \text { DW } & 196.64 \\ \text { DE } & 6.800 \text { usec } \\ \text { TE } & 6.50 \text { usec }\end{array}$

TE $\quad 298.4 \mathrm{~K}$

$\begin{array}{ll}\text { D1 } & 2.00000000 \mathrm{sec} \\ \text { D11 } & 0.03000000 \mathrm{sec}\end{array}$

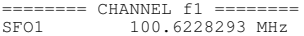

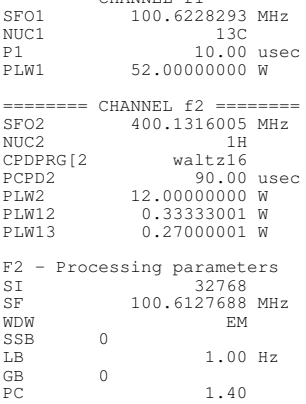

$\begin{array}{lllllllllllllllll}170 & 160 & 150 & 140 & 130 & 120 & 110 & 100 & 90 & 80 & 70 & 60 & 50 & 40 & 30 & 20 & \mathrm{ppm}\end{array}$ 
Ethyl (E)-2-(2-(ethylcarbamoyl)-4-phenyl-1-(4,4,5,5-tetramethyl-1,3,2-dioxaborolan-2-yl)but-1en-3-yn-1-yl) benzoate (5j)

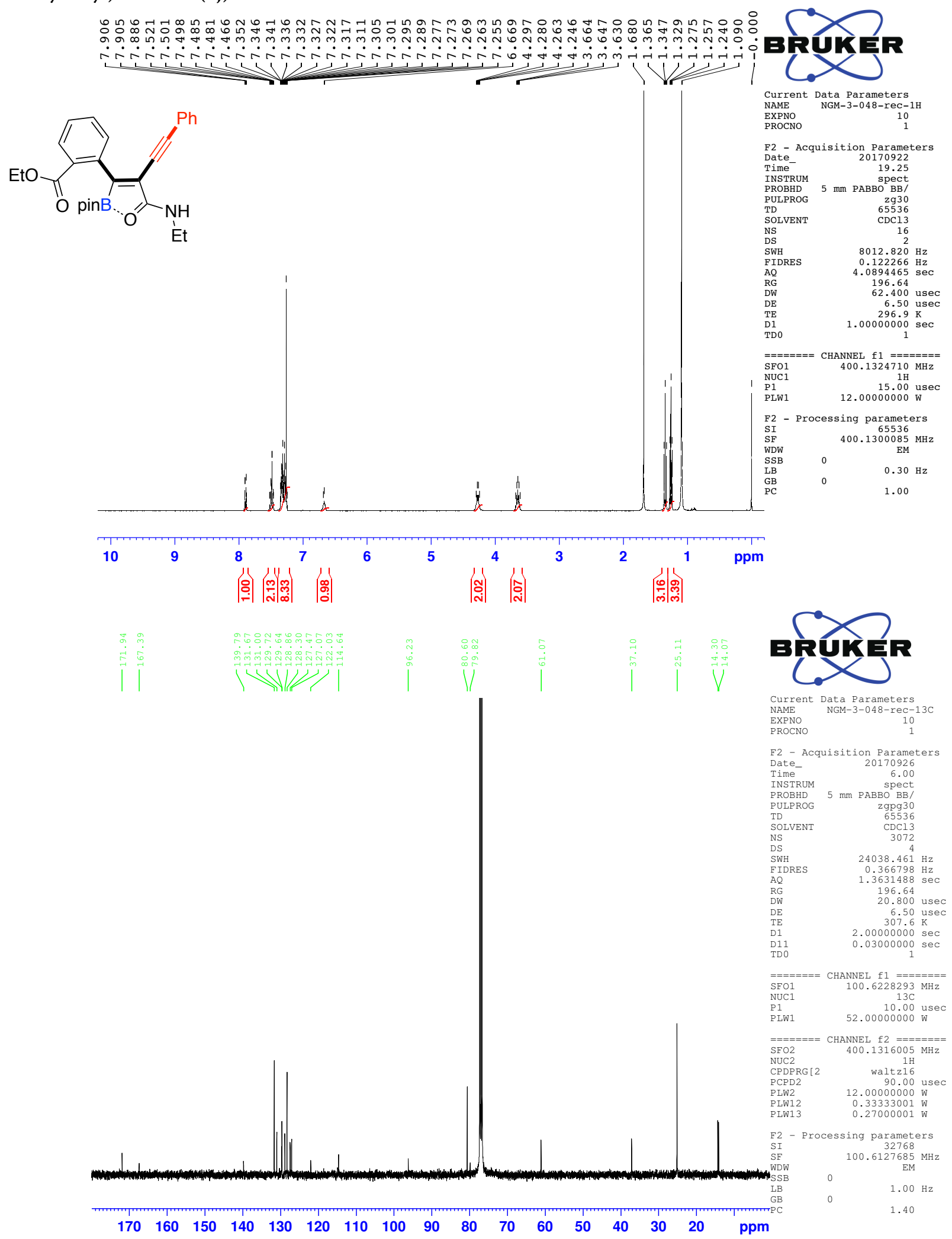

\section{S-58}


(E)-2-((4-cyanophenyl)(4,4,5,5-tetramethyl-1,3,2-dioxaborolan-2-yl)methylene)- $\mathrm{N}$-ethyl-4-phenylbut-3-ynamide (5k)
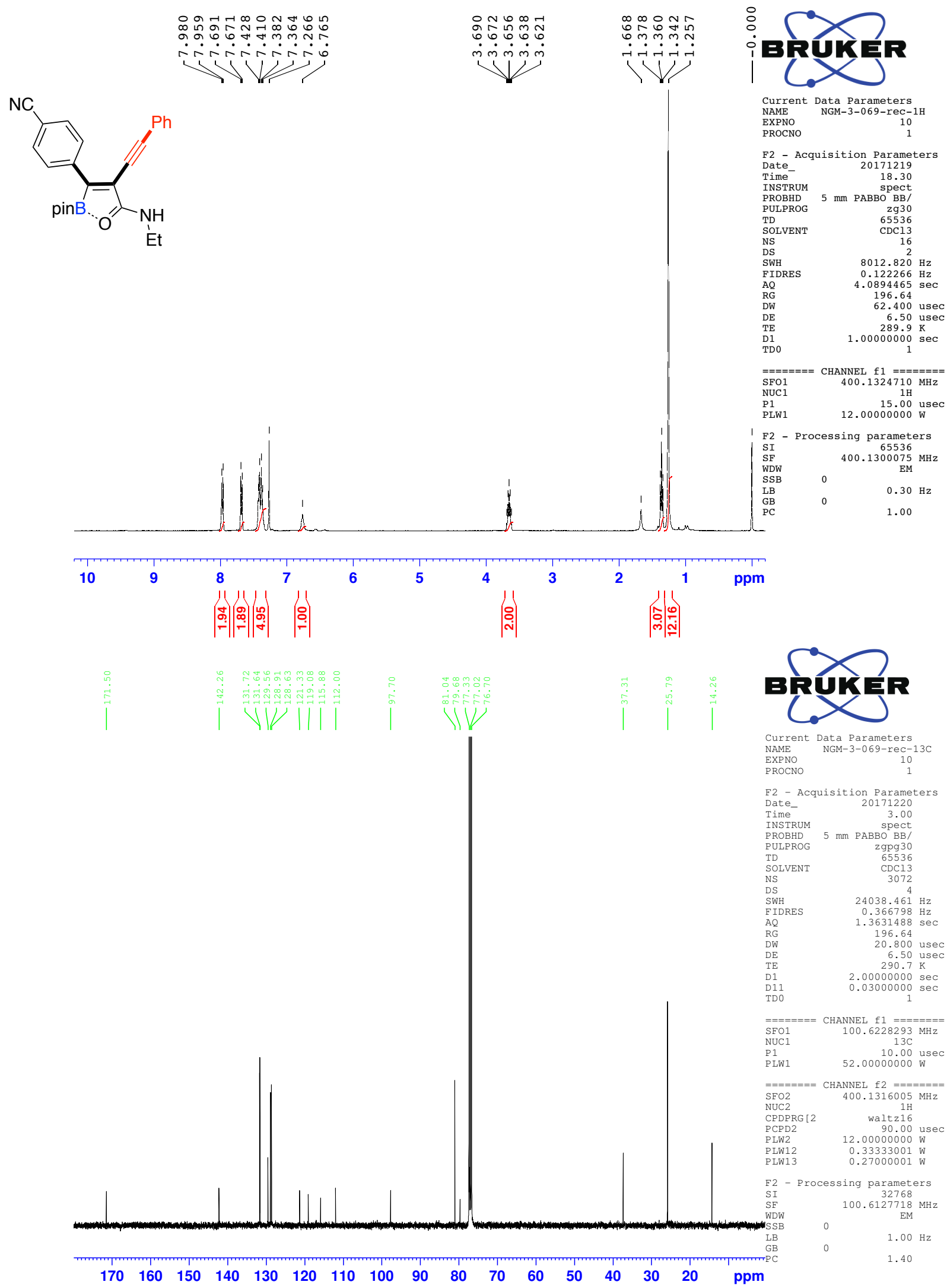

S-59 
(E)-N-ethyl-2-((4-nitrophenyl)(4,4,5,5-tetramethyl-1,3,2-dioxaborolan-2-yl)methylene)-4-phenylbut-3-ynamide (51)
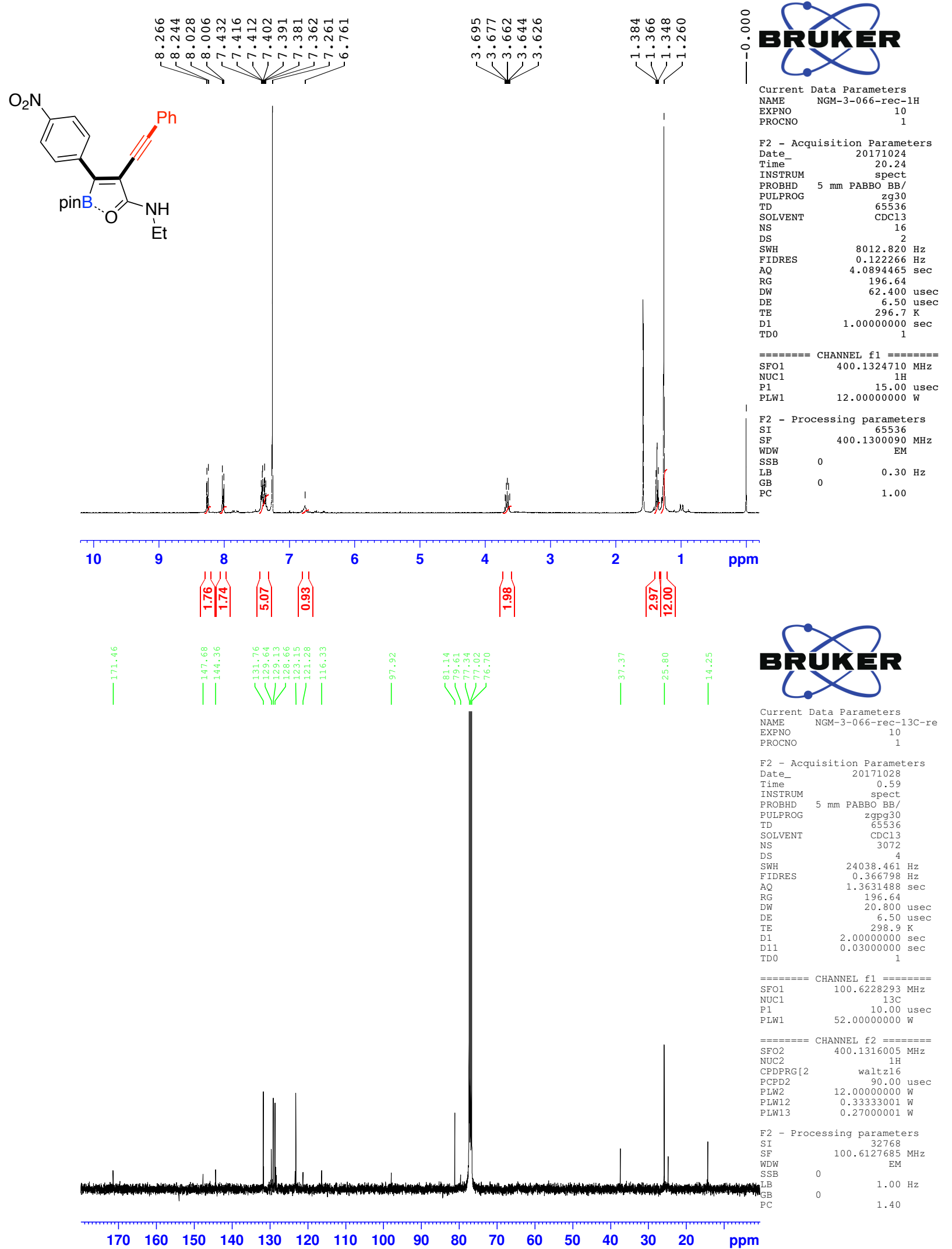

S-60 
(E)-N-ethyl-2-(mesityl(4,4,5,5-tetramethyl-1,3,2-dioxaborolan-2-yl)methylene)-4-phenylbut-3-ynamide (5m)
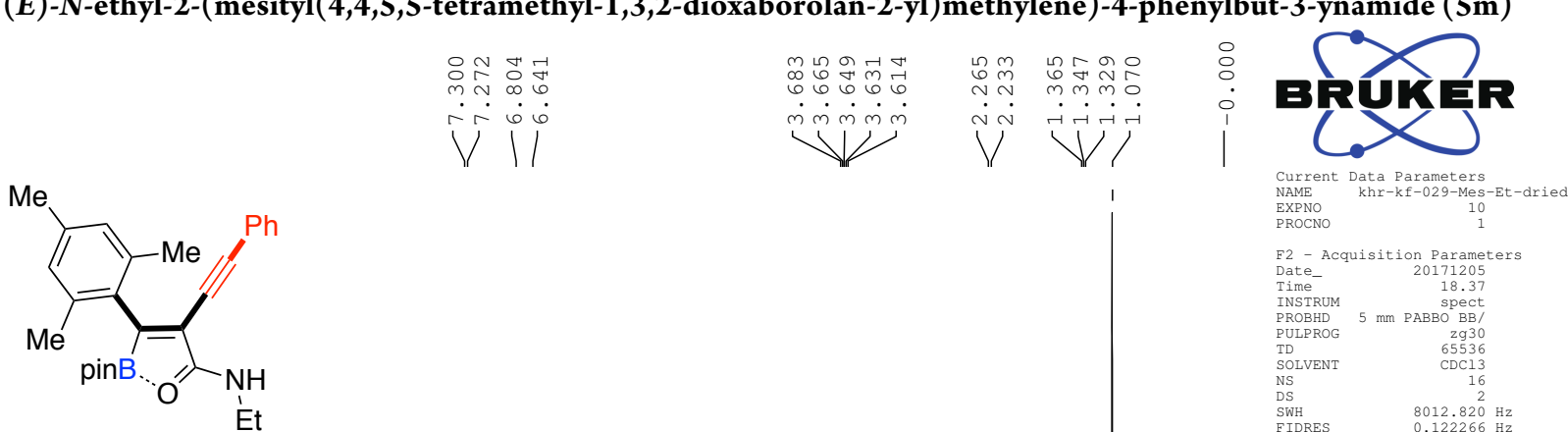

EXPNO

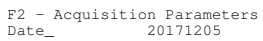

F2 - Acquisition Parame
Date_- 20171205
Time

$\begin{array}{lr}\text { Time } & 18.37 \\ \text { INSTRUM } & \text { spect } \\ \text { PROBDD } & 5 \mathrm{~mm} \text { PABBO BB } \\ \text { PUIPROG } & \text { zg } 30\end{array}$

PULPRO
TD
SDVEN
NS

$\begin{array}{lr}\text { NS } & 16 \\ \text { DS } & 2 \\ \text { SWH } & 8012.820 \mathrm{~Hz} \\ \text { FTDRES } & 0.122266 \mathrm{~Hz}\end{array}$

$\begin{array}{ll}\text { PIDRES } & 0.122266 \mathrm{~Hz} \\ \mathrm{AQ} & 4.0894465 \mathrm{sec}\end{array}$

$\begin{array}{lr}\text { RG } & 97.12 \\ \mathrm{DW} & 62.400 \mathrm{usec} \\ \mathrm{DE} & 6.50 \mathrm{usec} \\ \mathrm{TE} & 292.3 \mathrm{~K}\end{array}$

D1

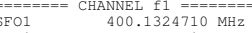

No

P1

$1 \mathrm{H}$
$15.00 \mathrm{use}$
$1.00000000 \mathrm{~W}$

F2 - Processing parameter

$\begin{array}{ll}\text { SI } & 65536 \\ \text { SE } & 400.1300074 \mathrm{MHz}\end{array}$
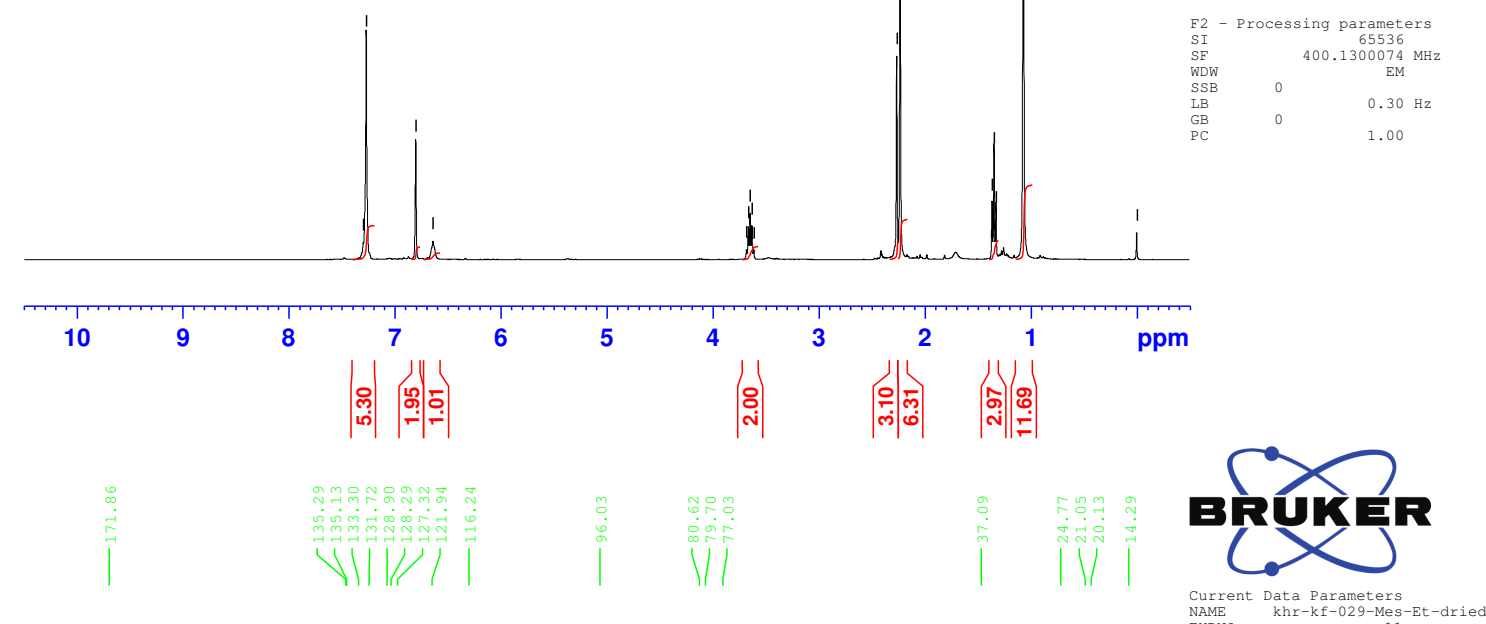

NAME
EXPNO
PROCNO

F2 - Acquisition Parameters

Time

3.03
3.03

ROBHD $5 \mathrm{~mm}$ PABBO BB/
RULPROG

PU⿴囗十)

NS
DS
SWH

$\begin{array}{lr}24038.461 \mathrm{~Hz} \\ \text { IDRES } & 0.366798 \mathrm{~Hz}\end{array}$

$1.3631488 \mathrm{sec}$

$\begin{array}{lr}\text { RG } & 196.64 \\ \text { DW } & 20.800 \mathrm{usec} \\ \text { DE } & 6.50 \mathrm{usec} \\ \text { TE } & 293.0 \mathrm{~K} \\ \text { D1 } & 2.00000000 \mathrm{sec} \\ \text { D11 } & 0.03000000 \mathrm{sec}\end{array}$

$\begin{array}{lr}\text { DW } & 20.800 \text { usec } \\ \text { DE } & 6.50 \text { usec } \\ \text { TE } & 293.0 \mathrm{~K} \\ \text { D1 } & 2.00000000 \text { sec } \\ \text { D11 } & 0.03000000 \mathrm{sec}\end{array}$

$=====$ CHANNEL $\mathrm{f} 1 \quad======+$
$\quad 100.6228293 \mathrm{MHz}$

1

$\begin{aligned} 10.00 & \text { use } \\ 52.00000000 \mathrm{~W} & \end{aligned}$

$\begin{array}{ll}======= & \text { CHANNEL } \mathrm{f} 2 \quad======= \\ \text { SFO2 } & 400.1316005 \mathrm{MHz}\end{array}$

waltz16

LW2 $12.00000000 \mathrm{us}$

$\begin{array}{ll} & 0.33333001 \mathrm{~W} \\ \text { PLW13 } & 0.27000001 \mathrm{~W}\end{array}$

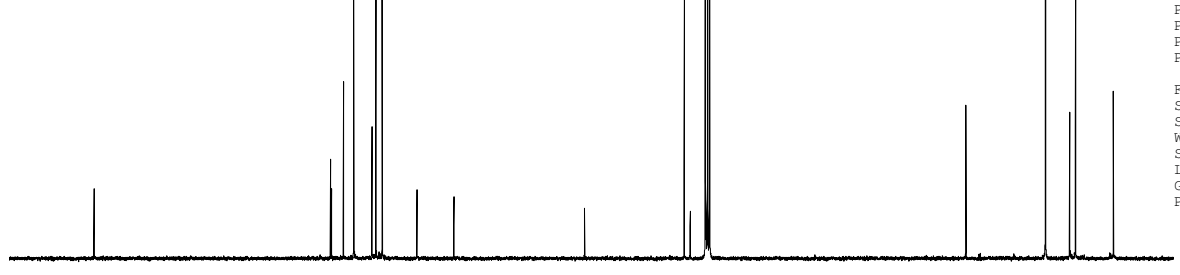

F2 - Processing parameters

$\begin{array}{cc}\text { SE } & 100.6127710 \\ \text { WW } & \text { MM }\end{array}$

$\mathrm{GB}$

$0 \quad 1.00$
1.40 
(E)-N-ethyl-4-phenyl-2-((4,4,5,5-tetramethyl-1,3,2-dioxaborolan-2-yl)(thiophen-3-yl)methylene)but-3-ynamide (5n)
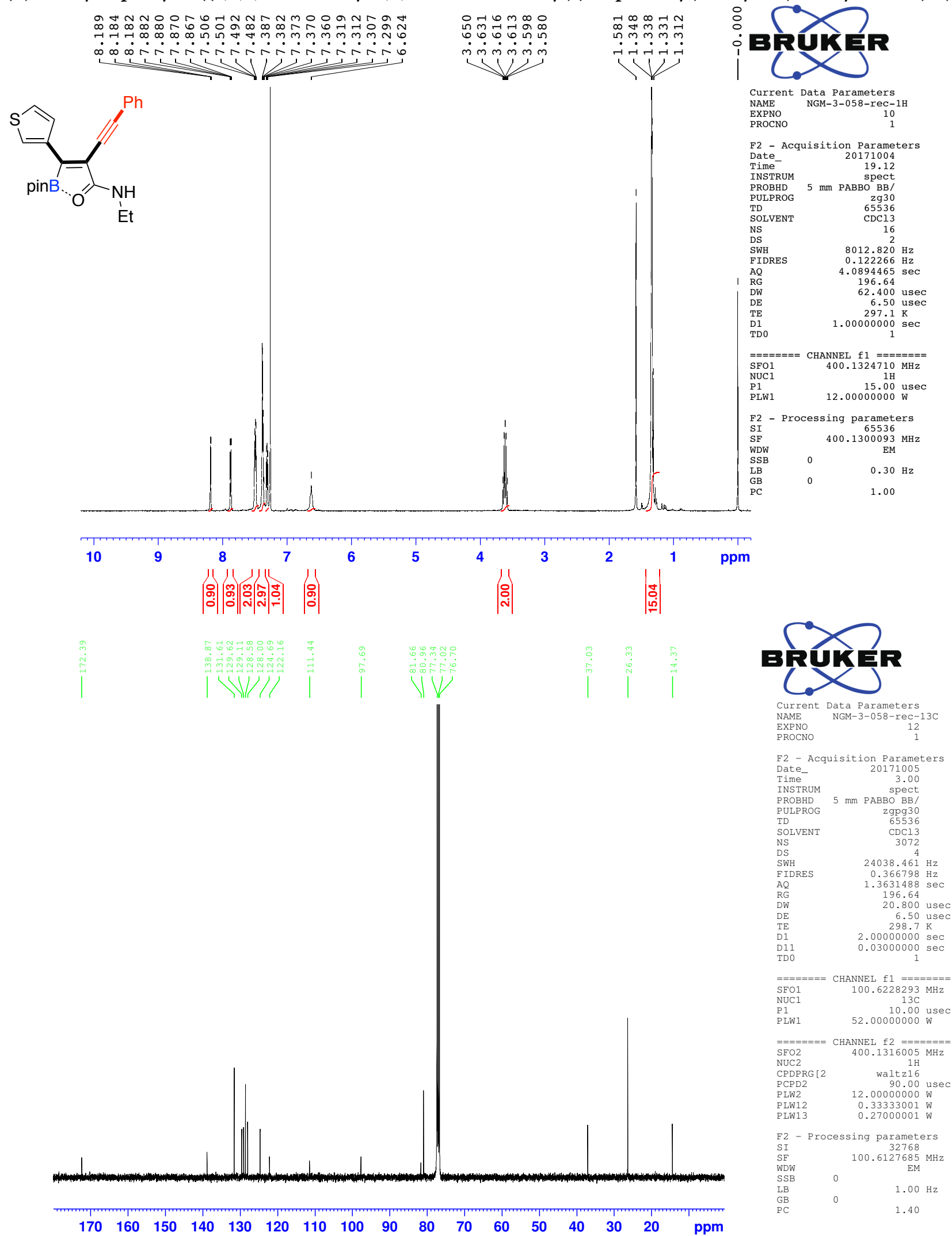
(E)-2-(benzo $[b]$ thiophen-2-yl(4,4,5,5-tetramethyl-1,3,2-dioxaborolan-2-yl)methylene)-N-ethyl-4-phenylbut-3-ynamide (5o)
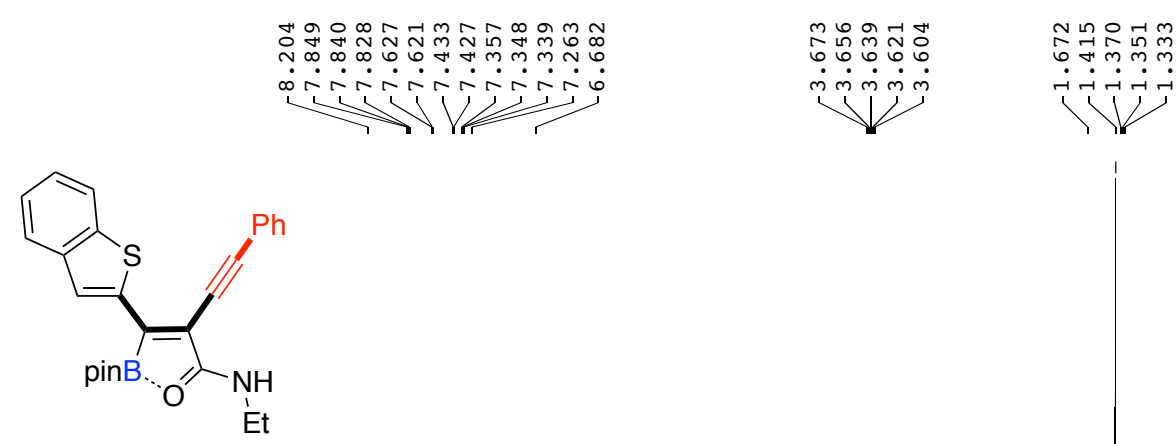

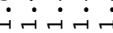

$\dot{m} \dot{m} \dot{m} \dot{~}$
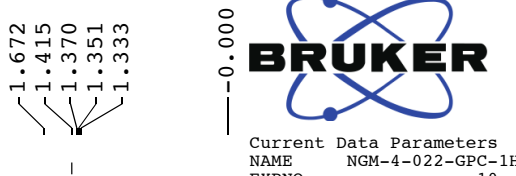
NAME
EXPNO

$\underset{20180125}{\text { F2 - Acquisition Parameters }}$

Date $\quad 20180125$

TIMSTRUM

PROBHD $5 \mathrm{~mm}$ PABBO BB/

PULPROG $5 \mathrm{~g} 30$

TD

$\begin{array}{lr}\text { SOLVENT } & \text { CDC13 } \\ \text { NS } & 16\end{array}$

$\begin{array}{lc}\text { NS } & 16 \\ \text { DS } & 2 \\ \text { SWH } & 8012.820 \mathrm{~Hz}\end{array}$

$\begin{array}{lr}\text { FIDRES } & 0.122266 \mathrm{~Hz} \\ \text { AQ } & 4.0894465 \mathrm{sec}\end{array}$

$\begin{array}{lr}\text { RG } & 196.64 \\ \text { DW } & 62.400 \text { usec } \\ \text { DE } & 6.50 \text { usec }\end{array}$

$\mathrm{DE}$
$\mathrm{TE}$
$\mathrm{TE}$

D1
TD0

$=======$ CHANNEL $f 11=======$
$400.1324710 \mathrm{MHz}$

$\begin{array}{lr}\text { NUC1 } & 1 \mathrm{H} \\ \text { P1 } & 15.00 \mathrm{usec} \\ \text { PLW1 } & 12.0000000 \mathrm{w}\end{array}$
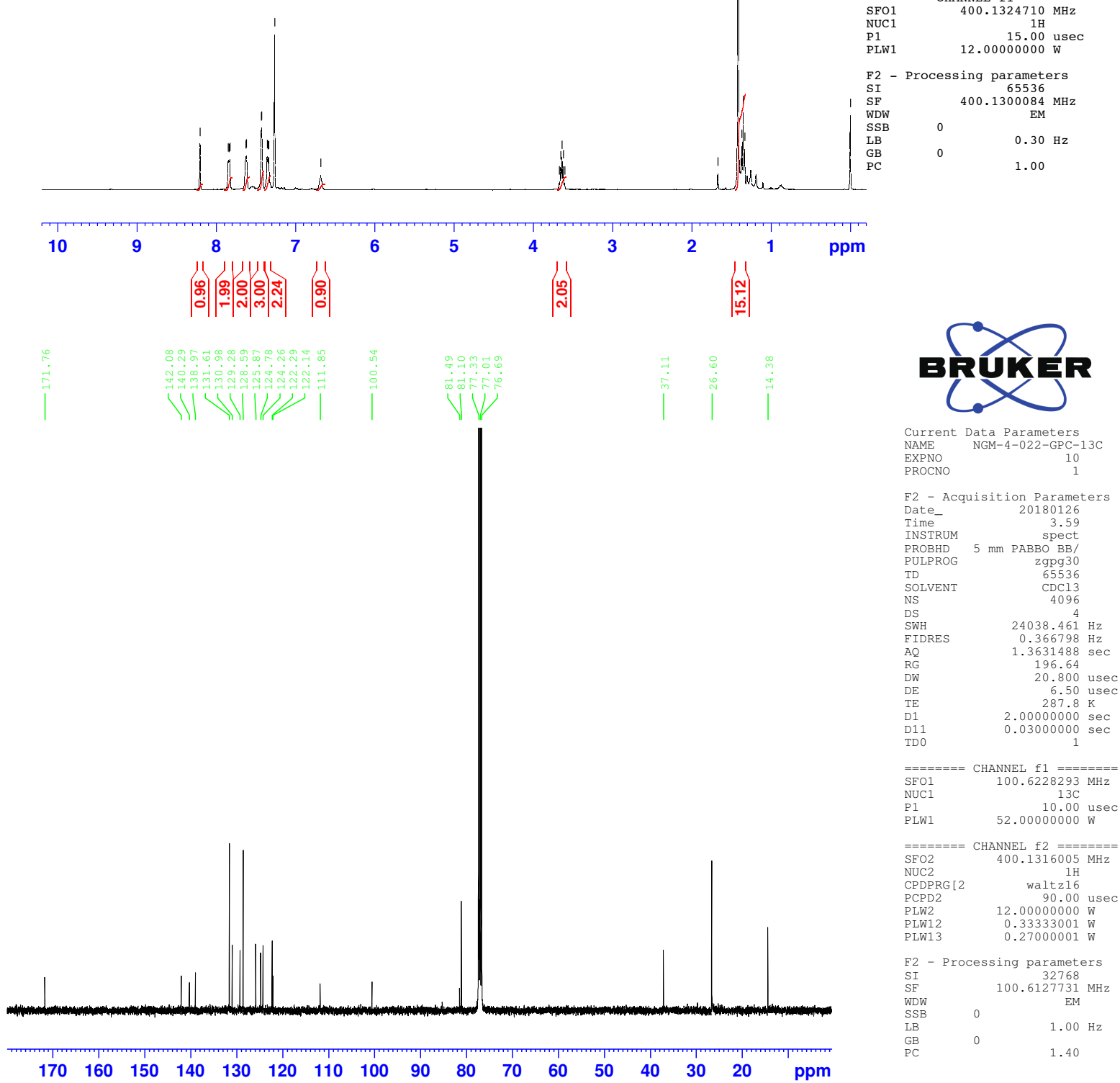
(E)-2-(dibenzo[b,d]thiophen-2-yl(4,4,5,5-tetramethyl-1,3,2-dioxaborolan-2-yl)methylene)-N-ethyl-4-phenylbut-3-ynamid $\mathbf{e}(\mathbf{5 p})$
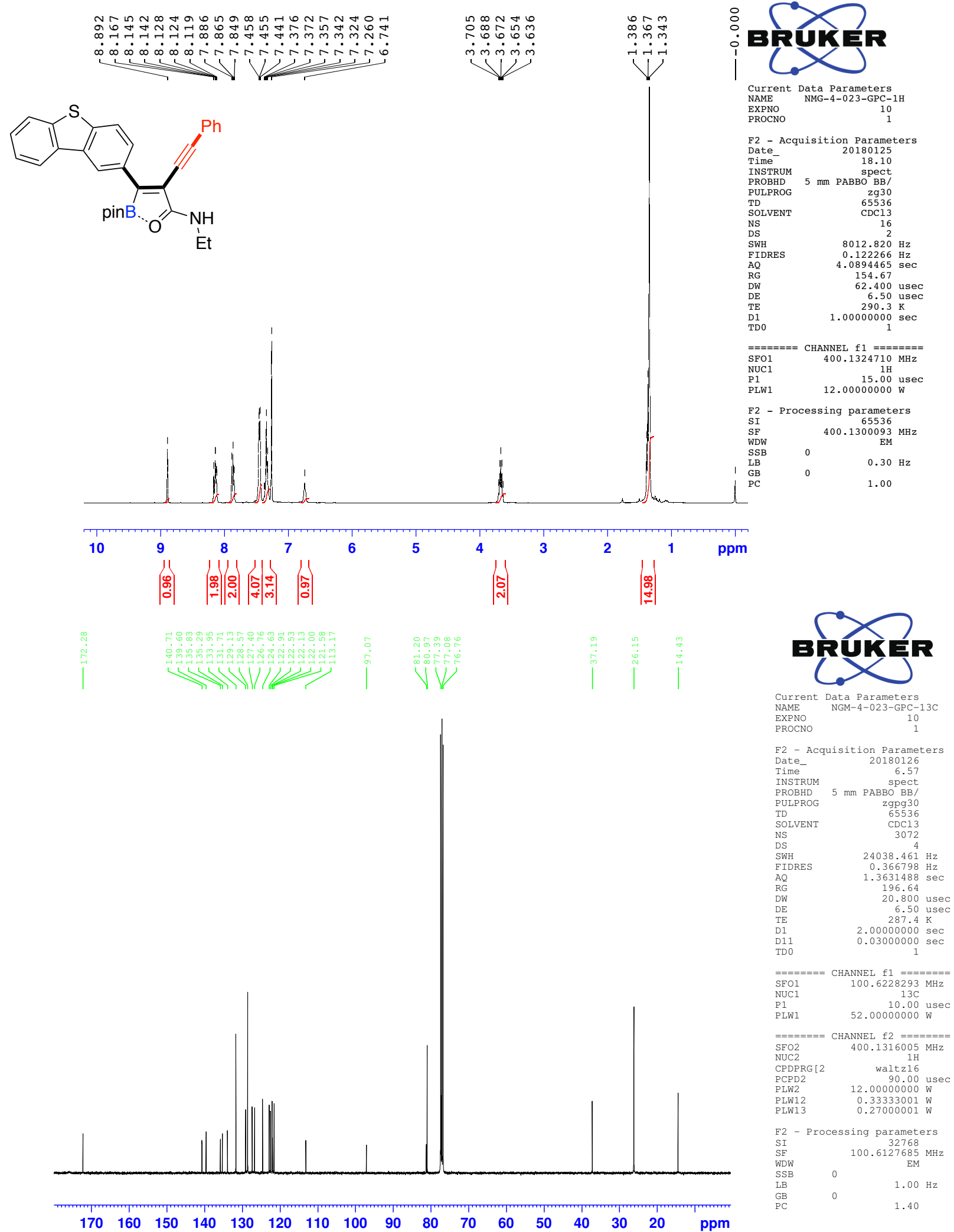
(E)-2-(cyclohex-1-en-1-yl(4,4,5,5-tetramethyl-1,3,2-dioxaborolan-2-yl)methylene)- $N$-ethyl-4-phenylbut-3-ynamide (5r)
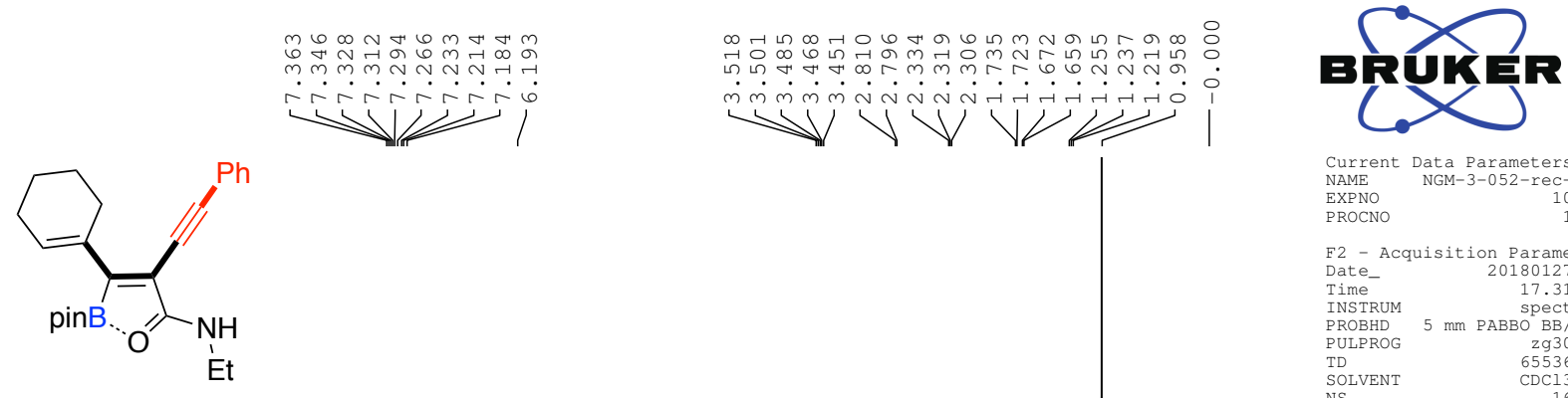

Current Data Parameters
NAME NGM-3-052-rec-1H

EXPNO
PROCNO

F2 - Acquisition Parameters

F2 - Acquisition Parame
Date-
Time

spect

PUOBHD

TD

TD
NS
NS

DS

DS
F IDRE
AQ

AQ
RG
DW

$\mathrm{DW}$
$\mathrm{DE}$
$\mathrm{TE}$
$\mathrm{D}$

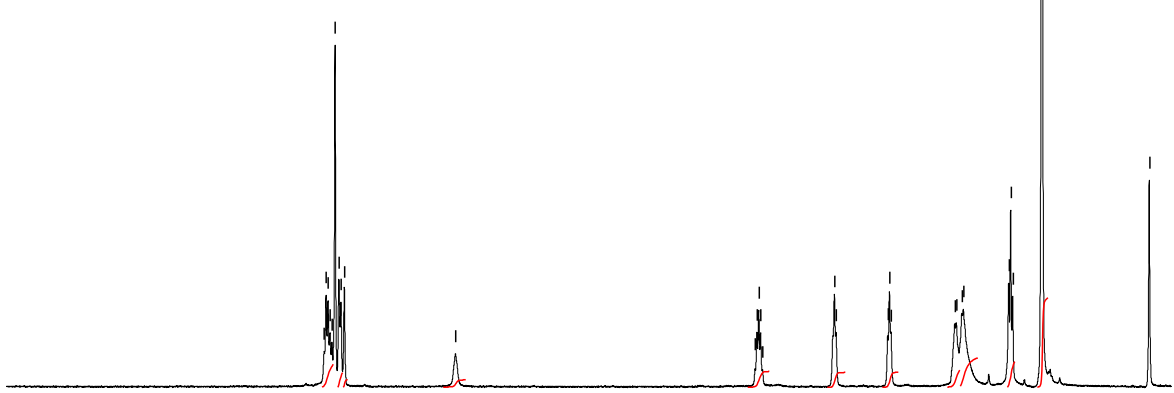

D1

$=======$ CHANNEL $\mathrm{f} 1 \mathrm{f}=======$
SFO1
$400.1324710 \mathrm{MHz}$

$\begin{array}{lrl}\text { NUC1 } & 1 \mathrm{H} \\ \text { P1 } & 15.00 \mathrm{use}\end{array}$

$\begin{array}{cc}\text { F2 } & \text { Processing parameters } \\ \text { SI } & 65536 \\ \text { SF } & 400.1300071 \mathrm{MH}\end{array}$

$\begin{array}{llr}\text { SF } & & 400.1300071 \\ \text { WDW } & & \text { EM } \\ \text { SSB } & 0 & 0.30 \mathrm{~Hz} \\ \text { LB } & 0\end{array}$

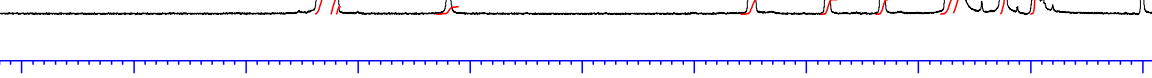

109

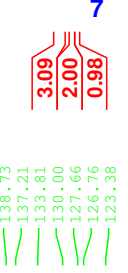

$\mid$ ș.

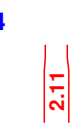

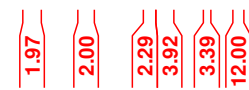
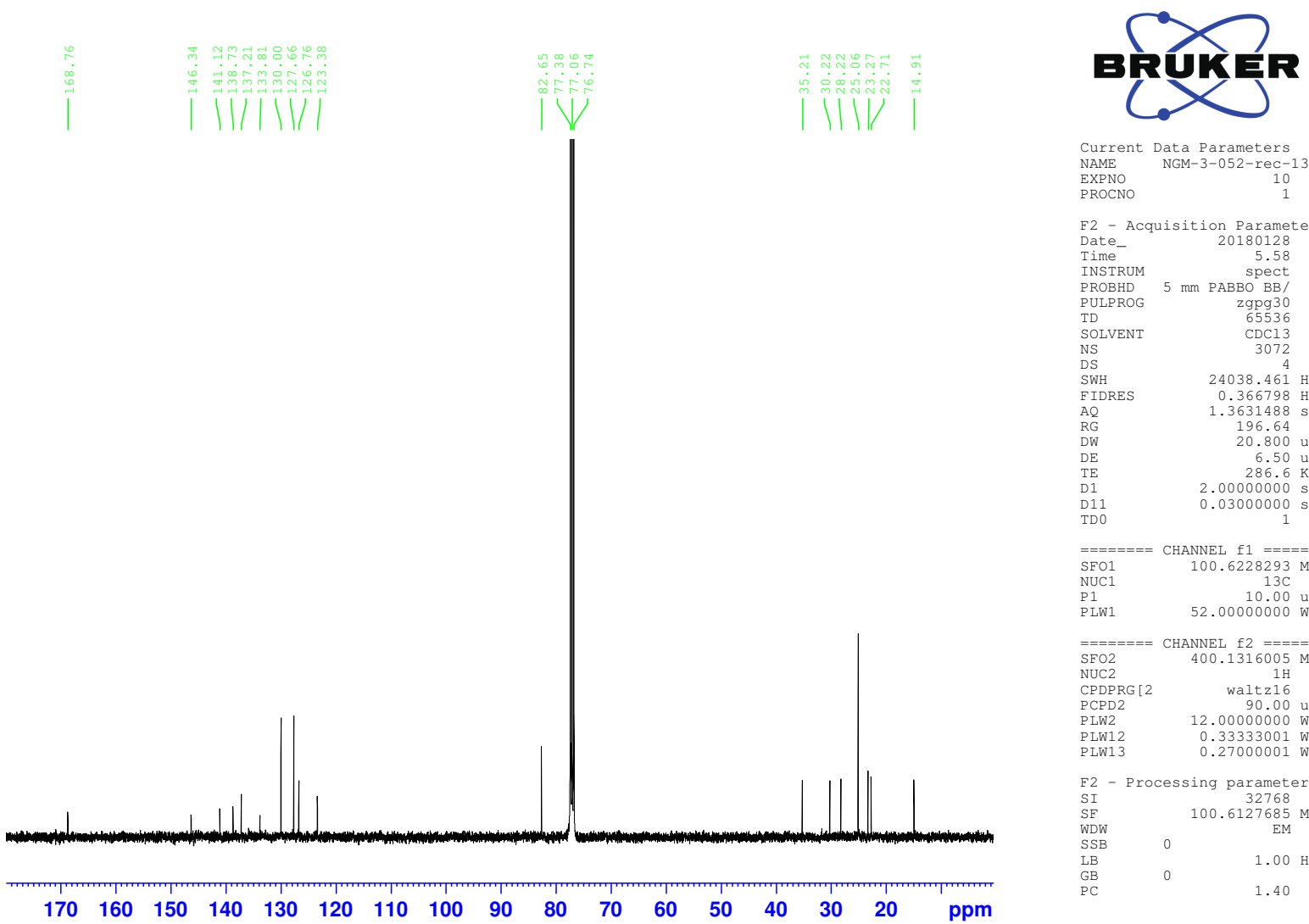

$\begin{array}{ll}\text { Current } & \text { Data Parameters } \\ \text { NAME } & \text { NGM-3-052-rec-13C }\end{array}$

EXPNO

F2 - Acquisition Parameters

Date
Time

Time

PROBHD
PULPROG

TD
SOLVEN

SOLV
NS

$\begin{array}{lr}\text { DS } & 4 \\ \text { SWH } & 24038.461 \mathrm{~Hz} \\ \text { FIDRES } & 0.366798 \mathrm{~Hz}\end{array}$

$\mathrm{AQ} \quad 1.3631488 \mathrm{sec}$

$\begin{array}{ll}\text { RG } & 196.64 \\ \text { DW } & 20.800 \mathrm{usec}\end{array}$

$\begin{array}{lr}\text { DE } & 6.50 \text { usec } \\ \text { TE } & 286.6 \mathrm{~K} \\ \text { D1 } & 2.0000000 \mathrm{sec}\end{array}$

$\begin{array}{ll}\text { D1 } & 2.00000000 \mathrm{sec} \\ \text { D11 } & 0.03000000 \mathrm{sec}\end{array}$

$=======$ CHANNEL $\mathrm{f} 1 \mathrm{f}=======$

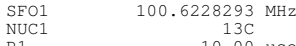

$\begin{array}{lr}\text { NUC1 } & 13 \mathrm{C} \\ \text { P1 } & 10.00 \mathrm{usec} \\ \text { PLW1 } & 52.00000000 \mathrm{~W}\end{array}$

$=======$ CHANNEL $\mathrm{f} 2 \quad========$
SFO2
$400.1316005 \mathrm{MHz}$

$\mathrm{SFO2} \quad 400.1316005$

$\begin{array}{lr}1 \mathrm{H} \\ \text { CPDPRG [2 } & \text { waltz16 } \\ \text { PCPD2 } & 90.00\end{array}$

$\begin{array}{lr}\text { PCPD2 } & 90.00 \text { us } \\ \text { PLW2 } & 12.00000000 \mathrm{~W}\end{array}$

PLW12 $\quad 0.33333001$

PLW13

$\begin{array}{lcc}\text { F2 } & \text { Processing parameters } \\ \text { SI } & 32768 \\ \text { SE } & 100.6127685 \mathrm{MHz} \\ \text { WDW } & & \text { EM } \\ \text { SSB } & 0 & 1.00 \mathrm{~Hz} \\ \text { LB } & 0 & \\ \text { GB } & 0 & \\ \text { PC } & & 1.40\end{array}$ 
(2E,4E)-N-ethyl-2-(phenylethynyl)-3-(4,4,5,5-tetramethyl-1,3,2-dioxaborolan-2-yl)-5-(4-(trifluoromethyl)phenyl)penta-2, 4-dienamide (5s)

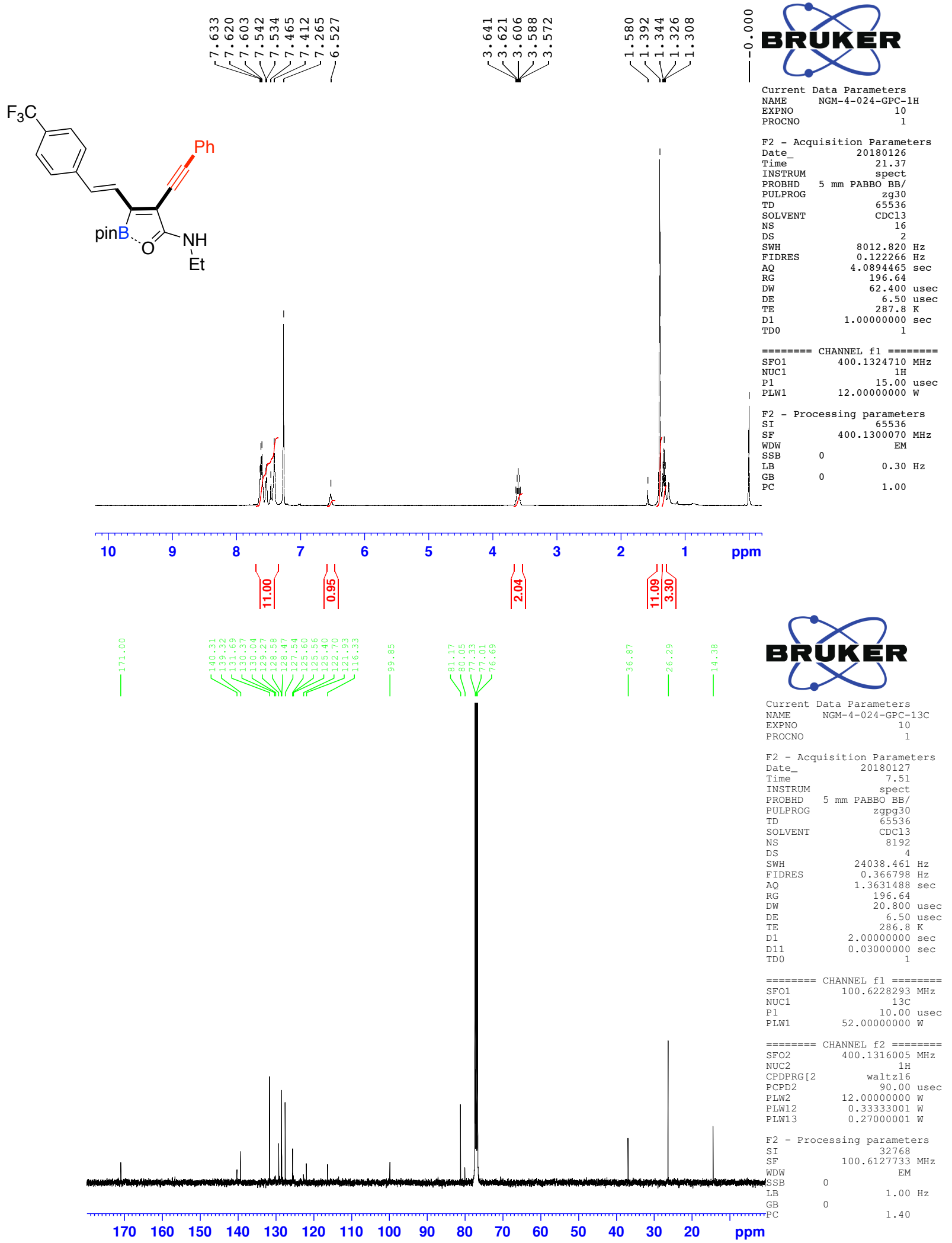


(E)-N-ethyl-2-(phenylethynyl)-3-(4,4,5,5-tetramethyl-1,3,2-dioxaborolan-2-yl)but-2-enamide (5t)
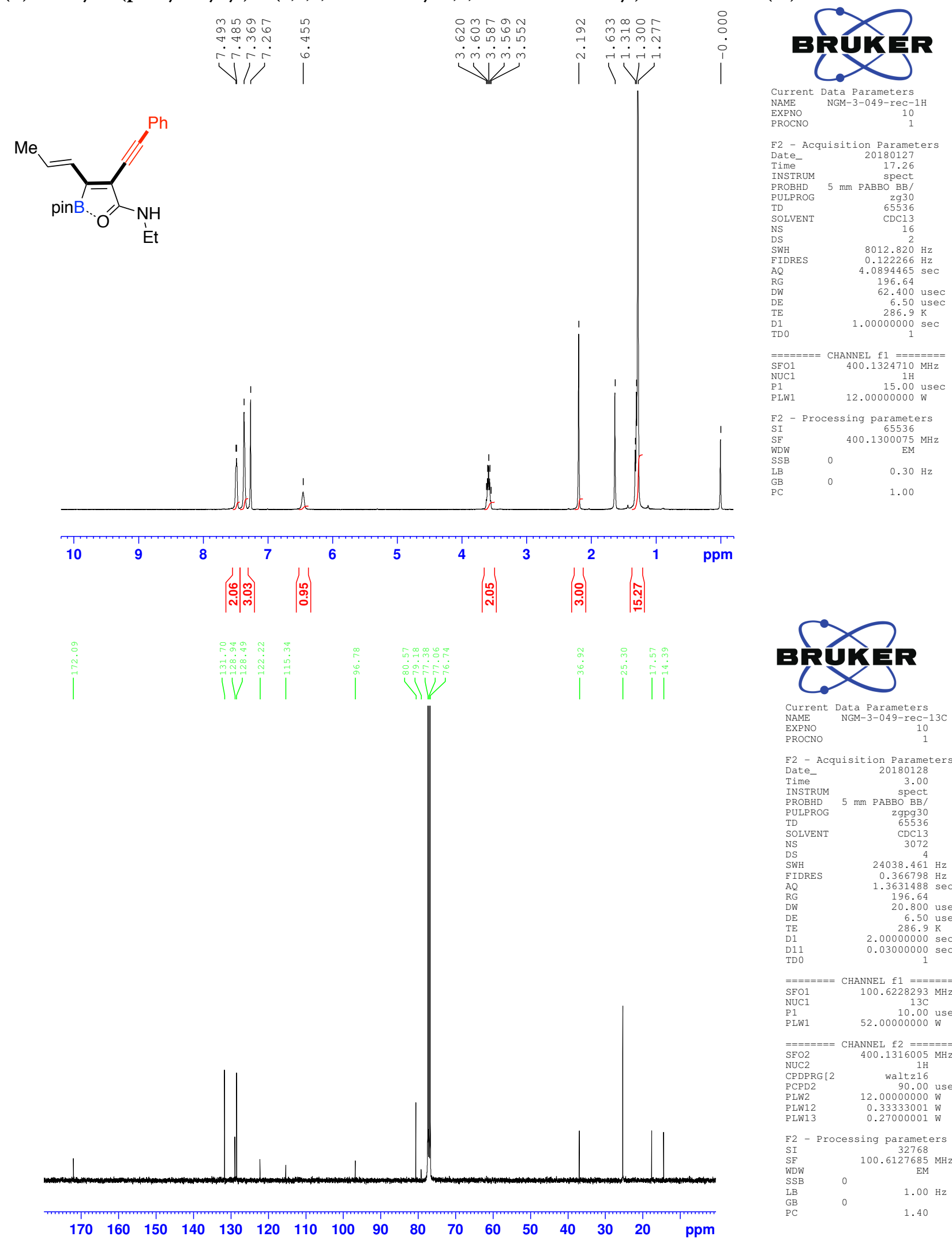

F2 - Acquisition Parameters

$\begin{array}{lr}\text { Date_- } & 20180128 \\ \text { Time } & 3.00\end{array}$

$\begin{array}{lr}\text { Time } & 3.00 \\ \text { INSTRUM } & \text { spect }\end{array}$

PROBHD $5 \mathrm{~mm}$ PABBO BB

PULPRO

SOLVEN

NS
DS
SWH

$\begin{array}{ll}\text { SWH } & 4 \\ & 24038.461 \mathrm{~Hz}\end{array}$

$\begin{array}{ll}\text { FIDRES } & 0.366798 \mathrm{~Hz} \\ \mathrm{AQ} & 1.3631488 \mathrm{sec}\end{array}$

$\begin{array}{lr}\text { RG } & 196.64 \\ \text { DW } & 20.800 \text { use }\end{array}$

DE
TE
DE.50 usec

$\begin{array}{ll}\text { D1 } & 2.00000000 \mathrm{sec} \\ \text { D11 } & 0.03000000 \mathrm{sec}\end{array}$

$=======$ CHANNEL fi $1========$

$=======$ CHANNEL $\mathrm{f} 1 \quad=======$
SFO1 $100.6228293 \mathrm{MHz}$
NUC1 $13 \mathrm{C}$
PUC

$\begin{array}{lr}\text { NUC1 } & 10.00 \text { usec } \\ \text { P1 } & 52.00000000 \mathrm{~W}\end{array}$

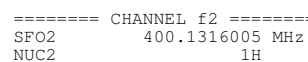

$\begin{array}{lr}\text { NUC2 } & 1 \mathrm{H} \\ \text { CPDPRG [2 } & \text { waltz16 } \\ \text { PCPD2 } & 90.00 \text { usec }\end{array}$

PLW2 $12.00000000 \mathrm{~W}$

$\begin{array}{ll}\text { PLW12 } & 0.33333001 \mathrm{~W} \\ \text { PLW13 } & 0.27000001 \mathrm{~W}\end{array}$

$\begin{array}{lcc}\text { F2 } & \text { - Processing parameters } \\ \text { SI } & 32768 \\ \text { SE } & 100.6127685 \\ \text { WDW } & & \text { EHz } \\ \text { SSB } & 0 & \\ \text { LB } & 0 & 1.00 \mathrm{~Hz} \\ \text { GB } & 0 & 1.40 \\ \text { PC } & & \end{array}$ 
(E)-N-ethyl-5-methoxy-2-(phenyl(4,4,5,5-tetramethyl-1,3,2-dioxaborolan-2-yl)methylene)pent-3-ynamide (6a)
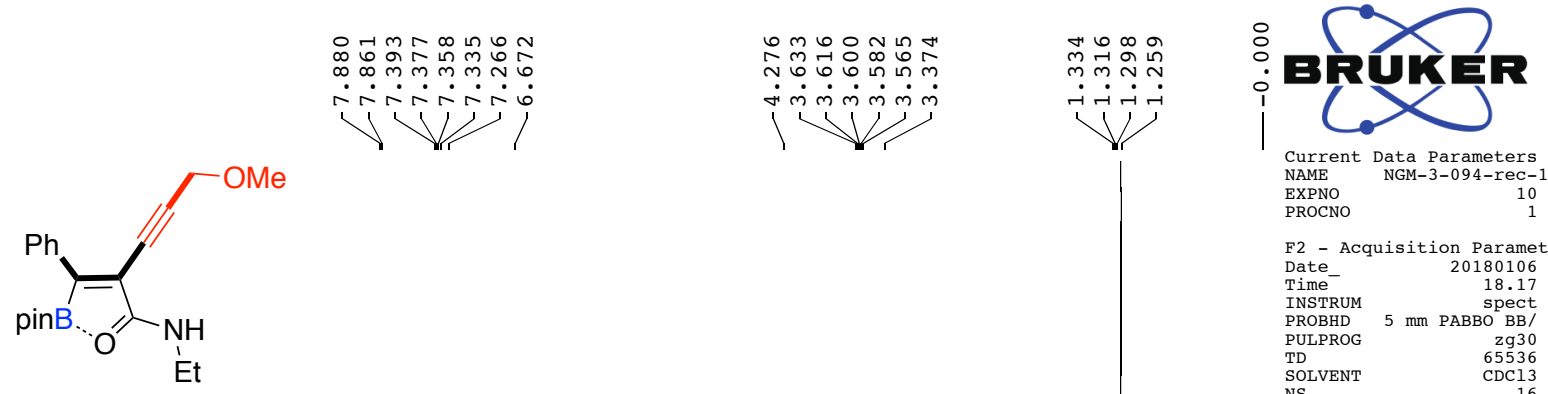

Current Data Parameters EXPNO

F2 - Acquisition Parameters
20180106

Date_ 20180106

Time

PROBHD $5 \mathrm{~mm}$ spect

TD

SOLVEN'

NS
DS
SWH

FWH

AQ
RG
DW

DW
DE
TE
$D 1$

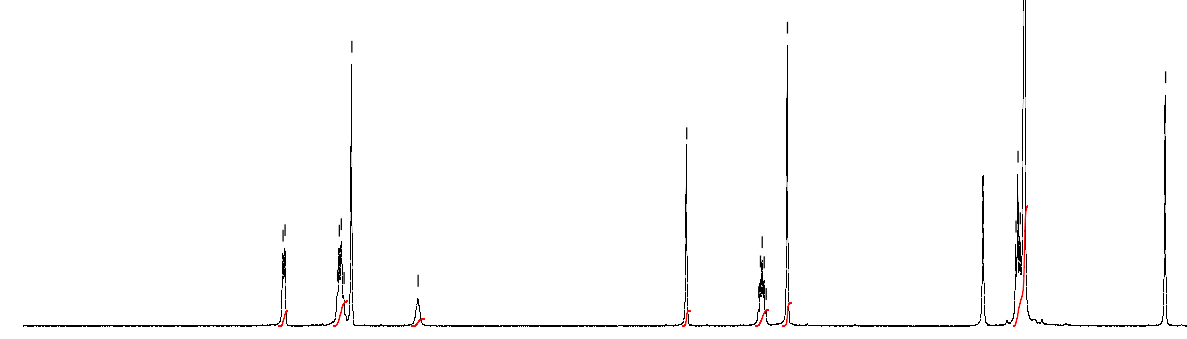

$=======$
CHANNEL $\mathrm{f} 1 \quad=======$
SFO1
NUC1
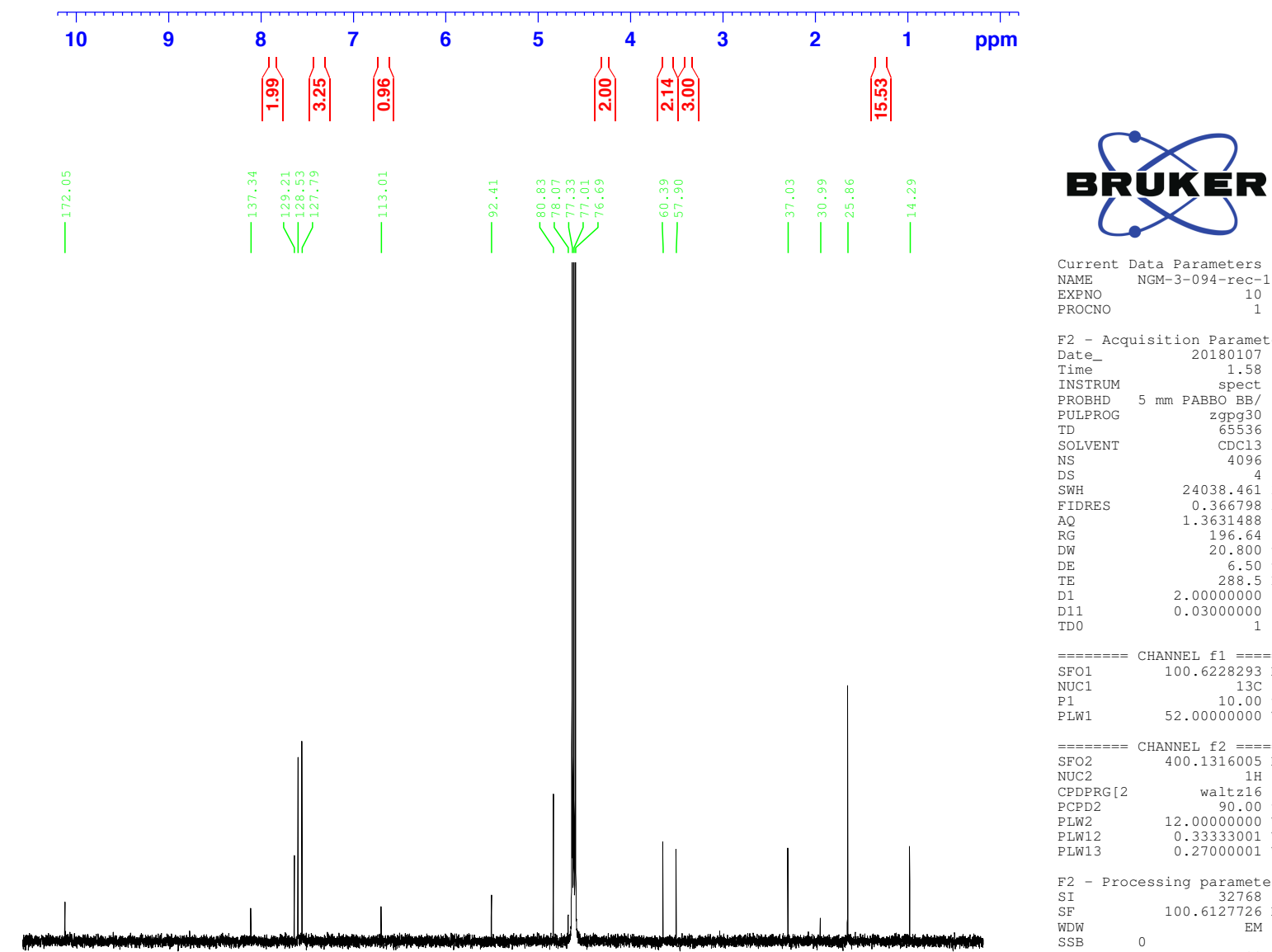

$\begin{array}{ll}\text { Current } & \text { Data Parameters } \\ \text { NAME } & \text { NGM-3-094-rec-13C }\end{array}$

EXPNO

EXPNO

F2 - Acquisition Parameters
Date- 20180107

$\begin{array}{lr}\text { Date__ } & 20180107 \\ & 1.58\end{array}$

Time

spect
PROBHD

PULPROG

TD

NS

DS

AQ $\quad 1.3631488 \mathrm{sec}$

RG

DE

D1

20.800 usec
6.50 usec

6.50 use
$288.5 \mathrm{~K}$

$2.00000000 \mathrm{sec}$

$=======$ CHANNEL $\mathrm{f} 1 \quad=======$

$\begin{array}{ll}\text { SFO1 } & 100.6228293 \mathrm{M} \\ \text { NUC1 } & \end{array}$

$\begin{array}{lr}\text { NUC1 } & 13 \mathrm{C} \\ \text { P1 } & 10.00 \mathrm{usec} \\ \text { PLW1 } & 52.00000000 \mathrm{~W}\end{array}$

$=======$ CHANNEL $\mathrm{f} 2=======$

SFO2 $\quad 400.1316005 \mathrm{MHz}$

$\begin{array}{lr}\text { NUC2 } & 1 \mathrm{H} \\ \text { CPDPRG } 2 & \text { waltz16 }\end{array}$

$\begin{array}{lr}\text { PCPD2 } & 90.00 \text { use }\end{array}$

$\begin{array}{lr}\text { PLW2 } & 12.00000000 \mathrm{~W} \\ \text { PLW12 } & 0.33333001 \mathrm{~W}\end{array}$

F2 - Processing parameter

SI Processing parameter

$\begin{array}{lc}\text { SF } & 100.6127726 \\ \text { WDW } & \text { MM }\end{array}$

$\begin{array}{lll}\mathrm{SSB} & 0 & 1.00 \mathrm{~Hz}\end{array}$

$\begin{array}{lllllllllllllllll}170 & 160 & 150 & 140 & 130 & 120 & 110 & 100 & 90 & 80 & 70 & 60 & 50 & 40 & 30 & 20 & \mathrm{ppm}\end{array}$

$\begin{array}{lll}\text { GB } & 0 & 1.40\end{array}$ 
(E)-7-chloro-N-ethyl-2-(phenyl(4,4,5,5-tetramethyl-1,3,2-dioxaborolan-2-yl)methylene)hept-3-ynamide (6b)
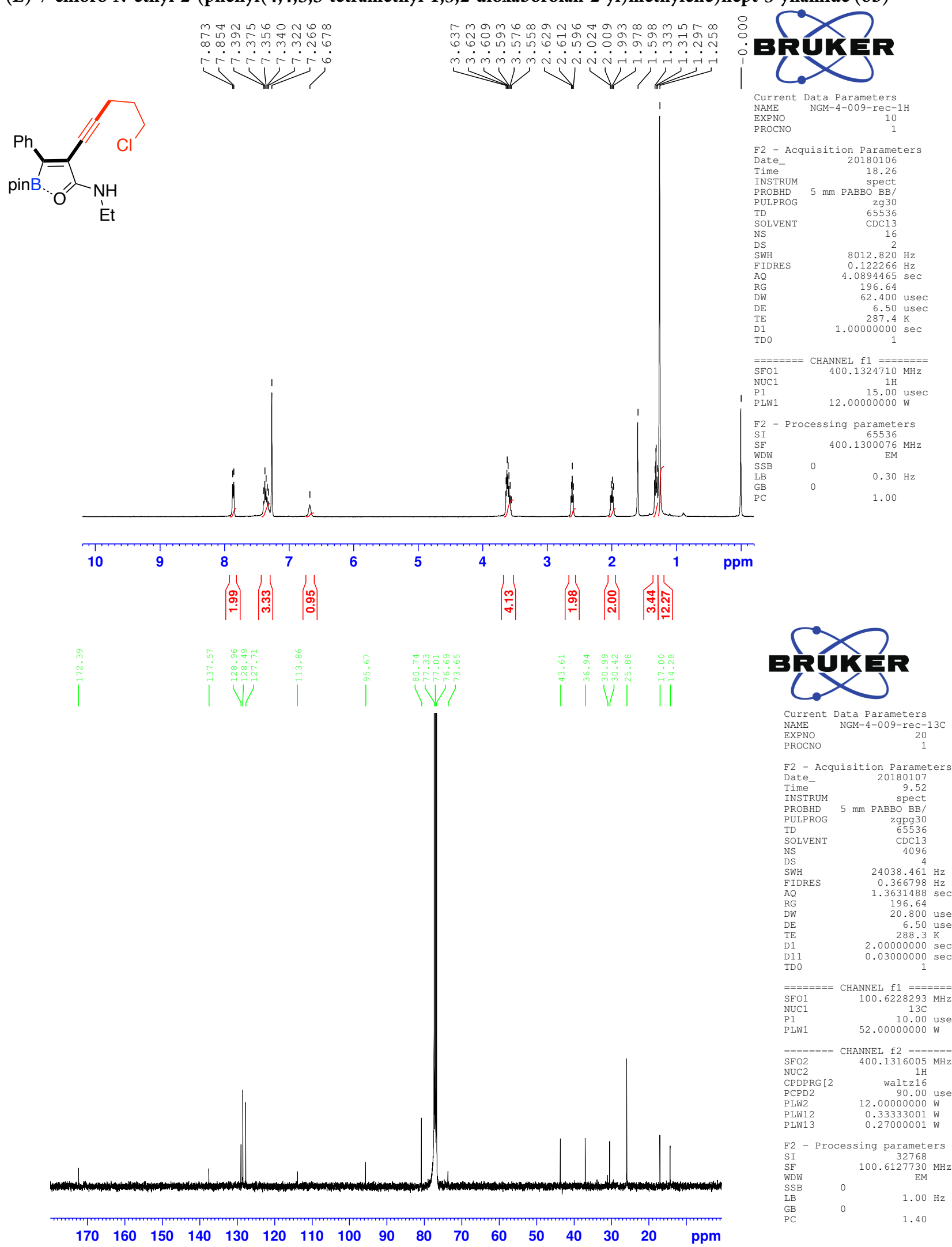

PROCNO

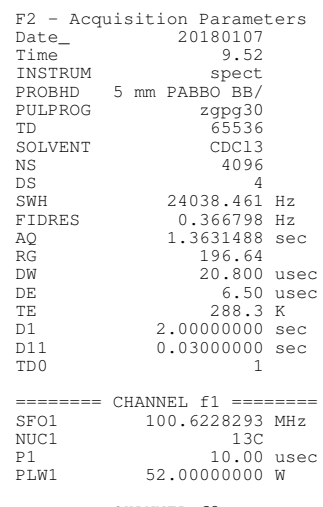

$=======$ CHANNEL f 2 $=======$

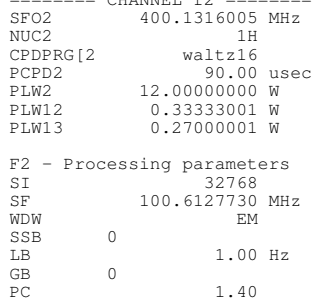


$(2 S, 3 S, 4 R, 5 S, 6 S)-2-($ acetoxymethyl)-6-(2-(4-(( $(E)-4-$ phenyl-2-(phenyl (4,4,5,5-tetramethyl-1,3,2-dioxaborolan-2-yl)methyl ene)but-3-ynamido)methyl)-1H-1,2,3-triazol-1-yl)ethoxy)tetrahydro-2H-pyran-3,4,5-triyl triacetate (7)

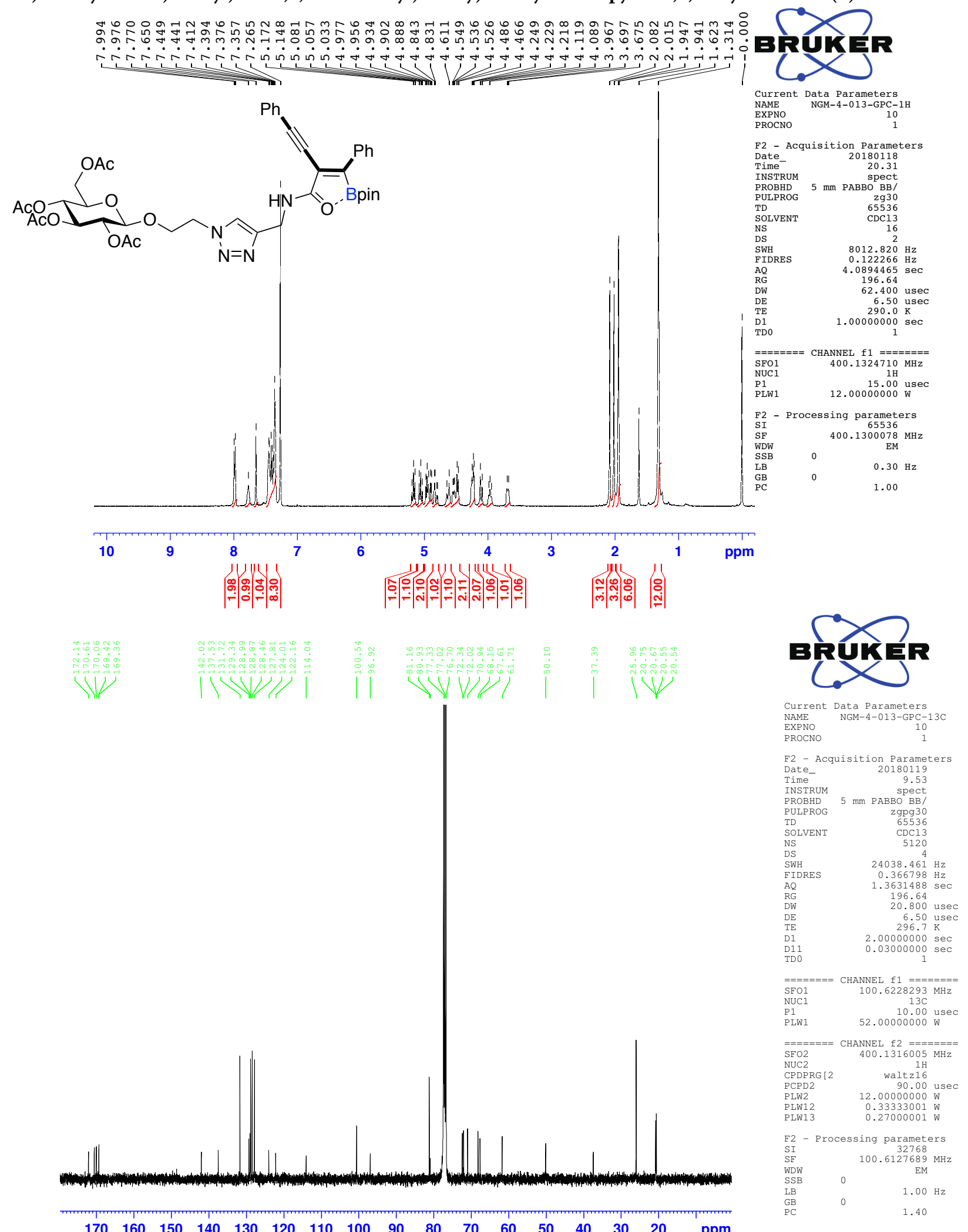


(E)-2-((difluoroboraneyl)(phenyl)methylene)- $\mathrm{N}$-ethyl-4-phenylbut-3-ynamide (8)

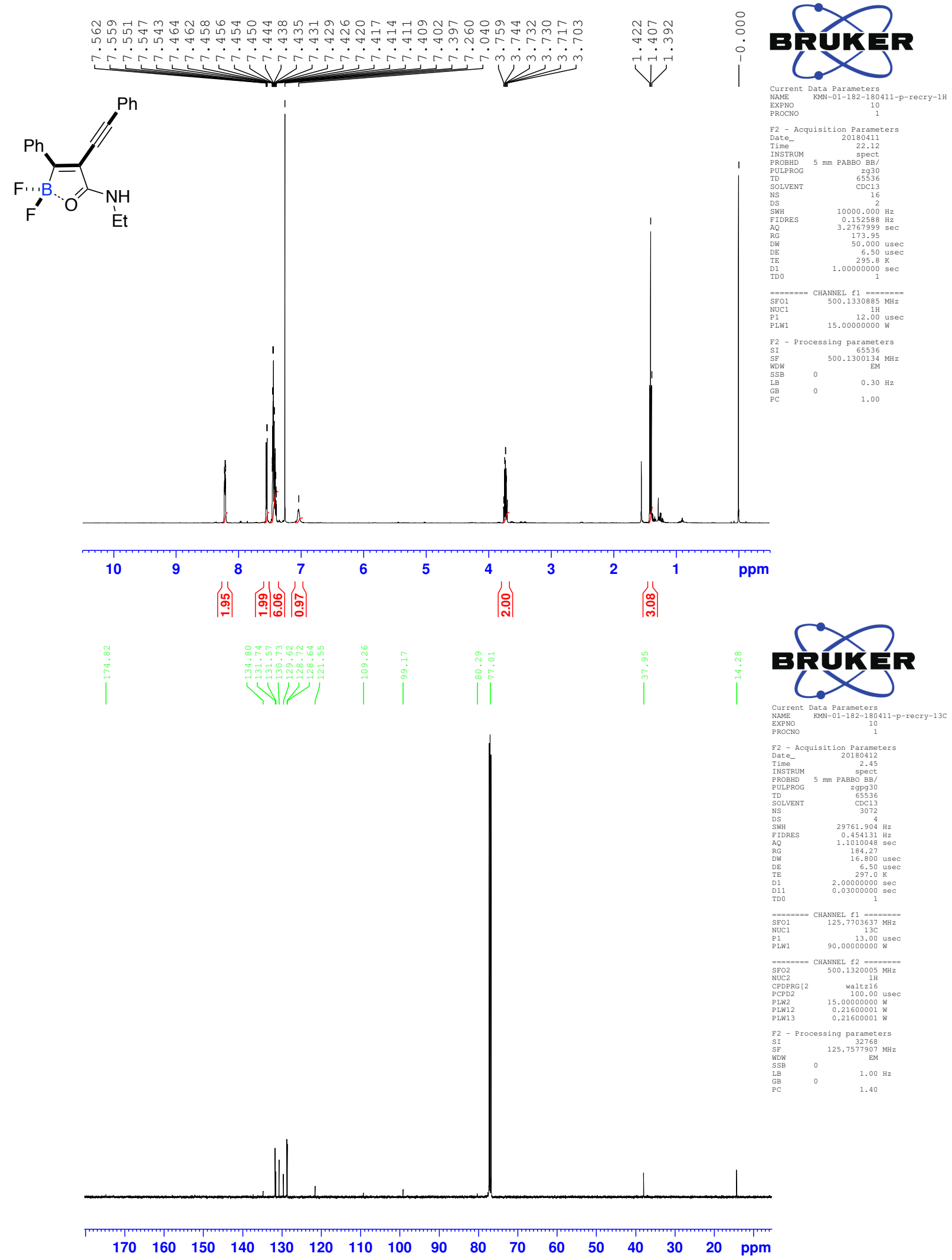

S-71 
$N$-isobutyl-3-phenylpropiolamide (1c)
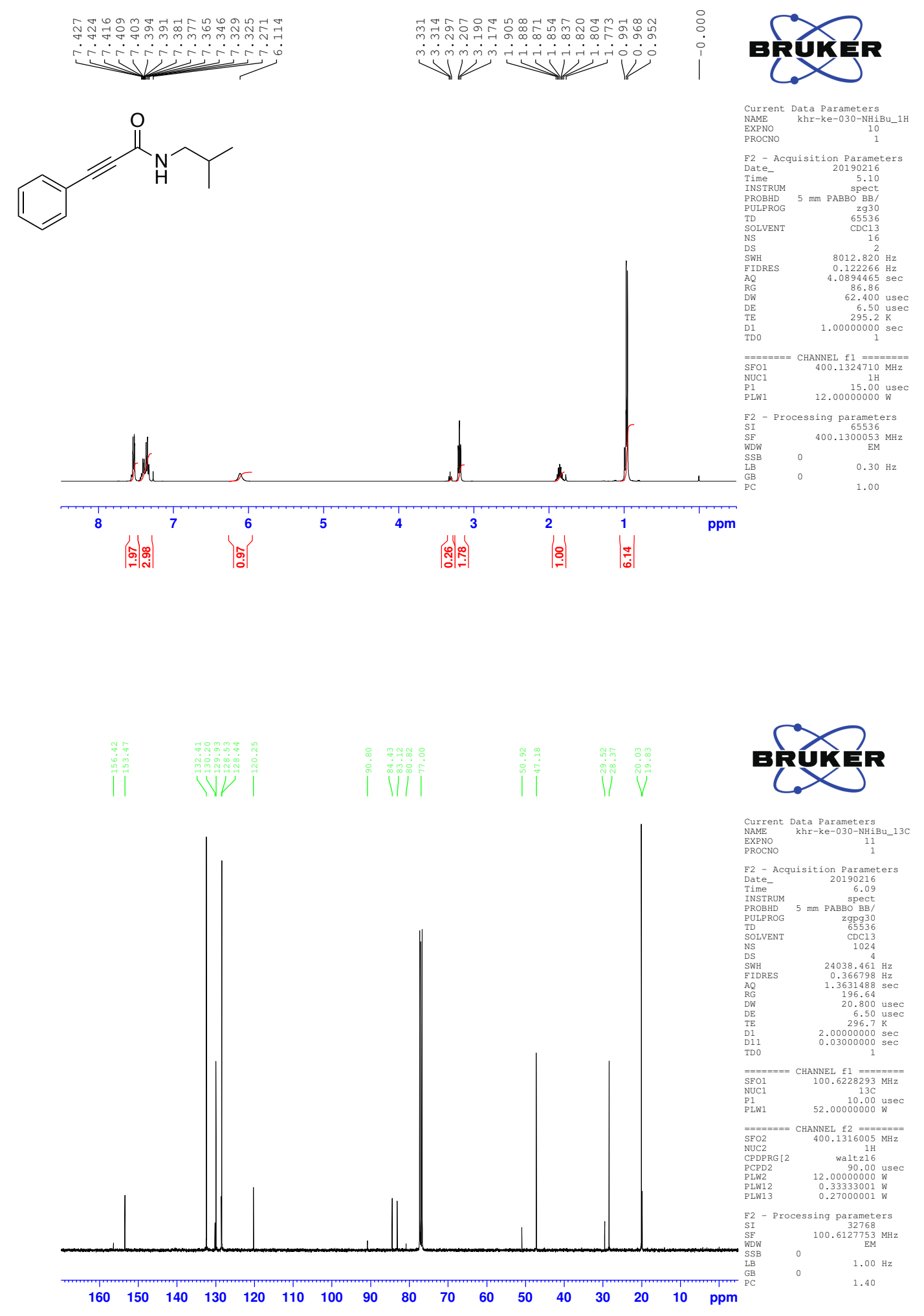

S-72 
N-cyclopropyl-3-phenylpropiolamide (1f)
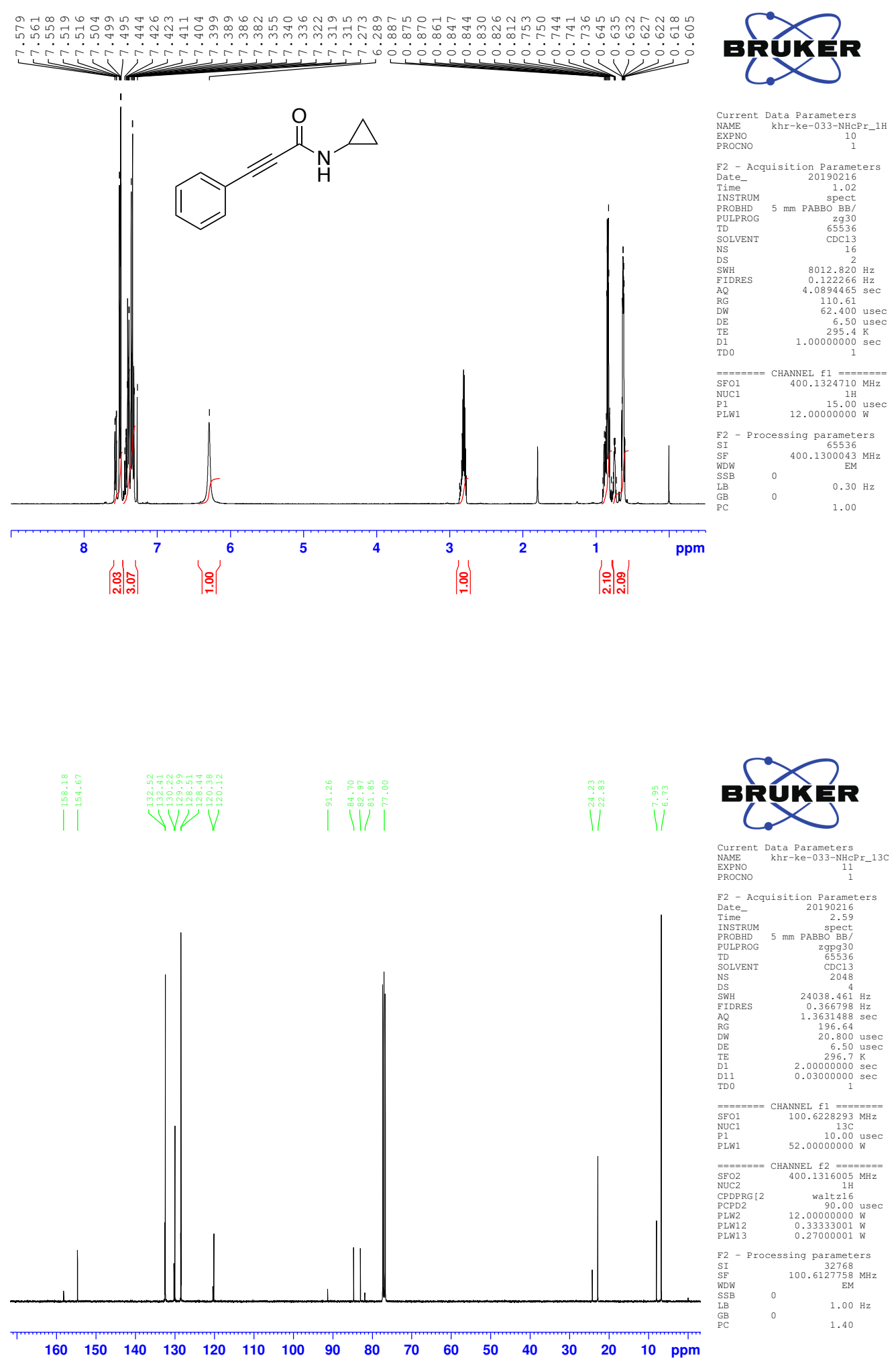
3-phenyl-N-(2-((triisopropylsilyl)oxy)ethyl)propiolamide (1i)
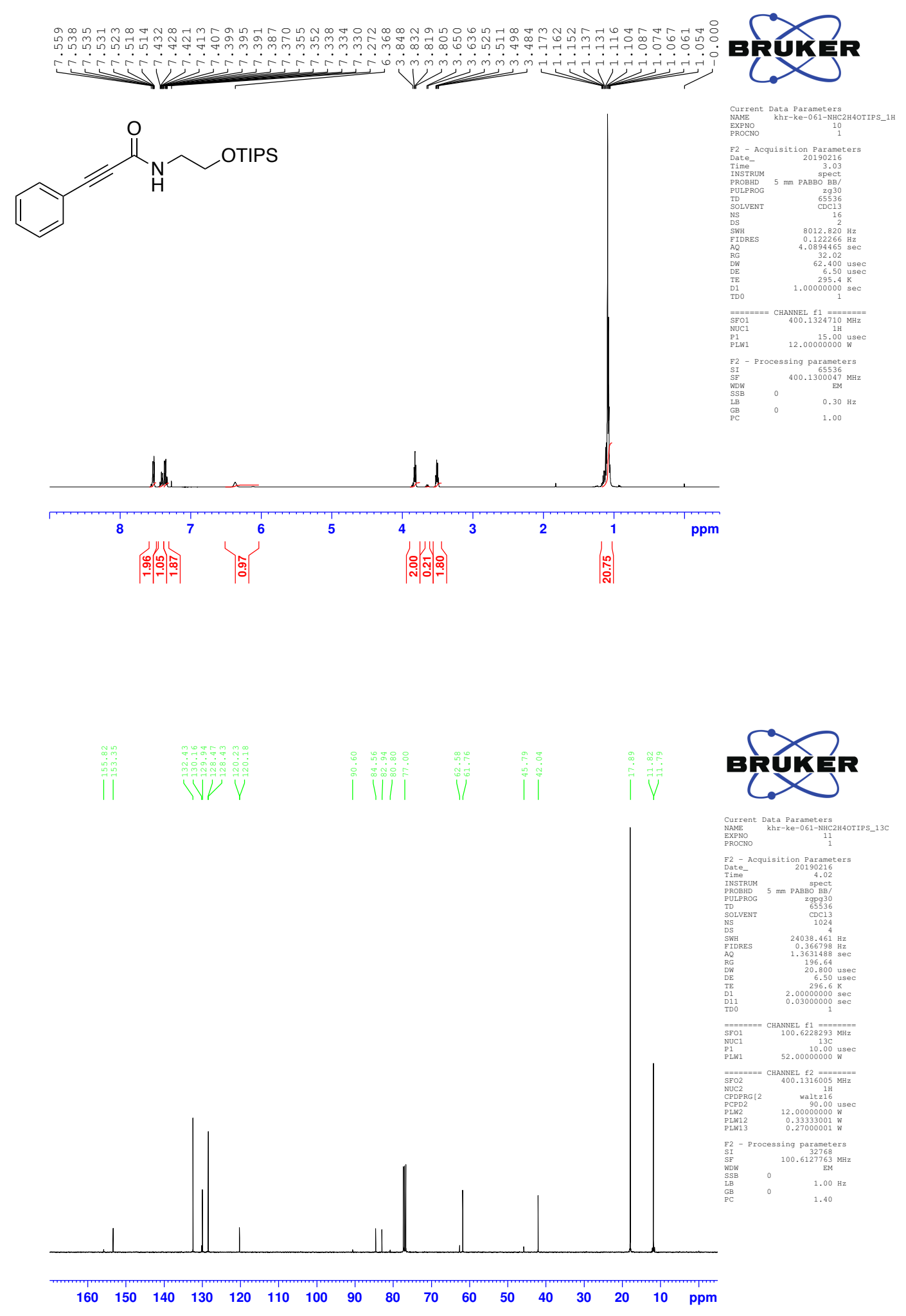
$N$-methoxy-3-phenylpropiolamide (1m)
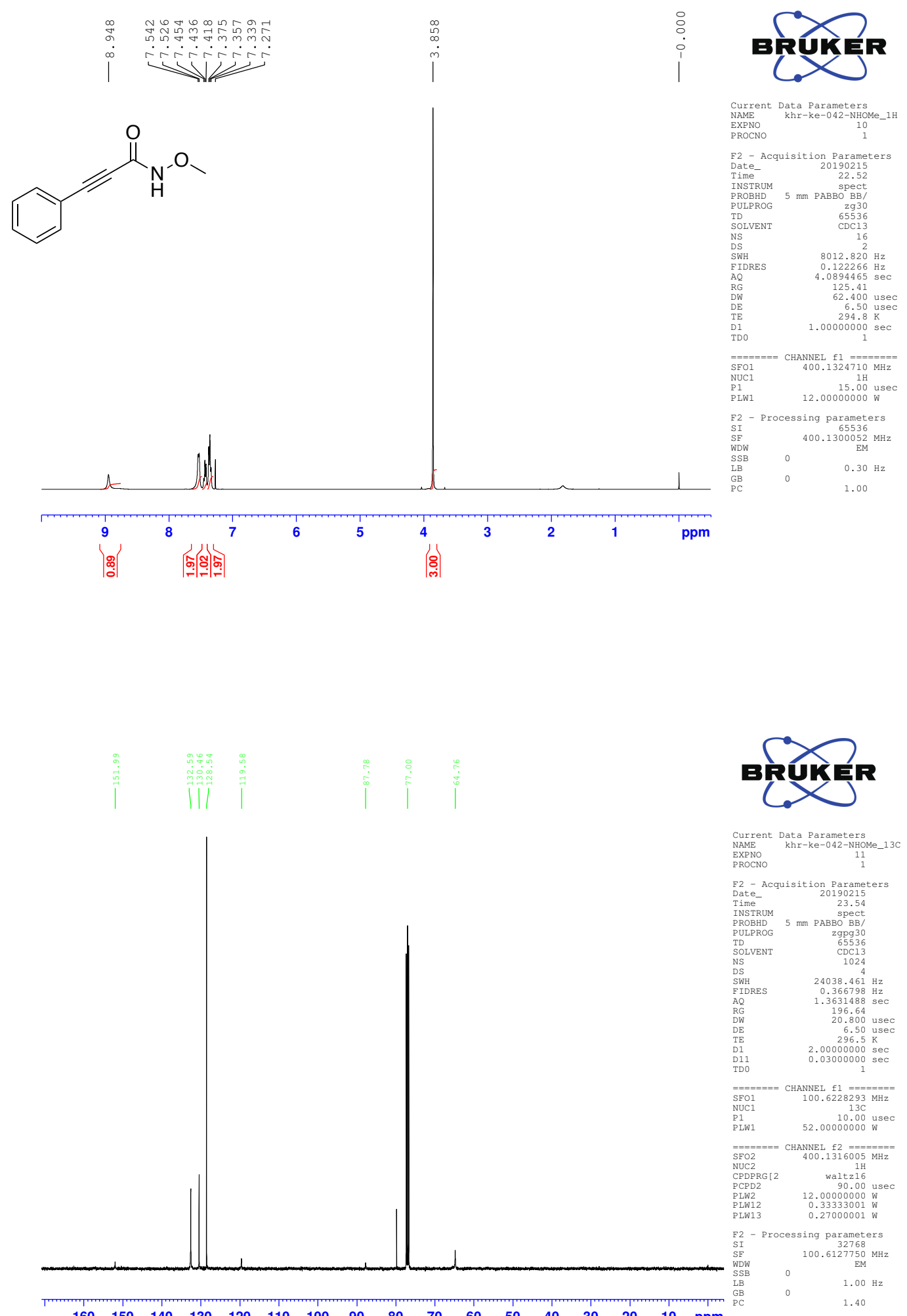
N-mesityl-3-phenylpropiolamide (1q)
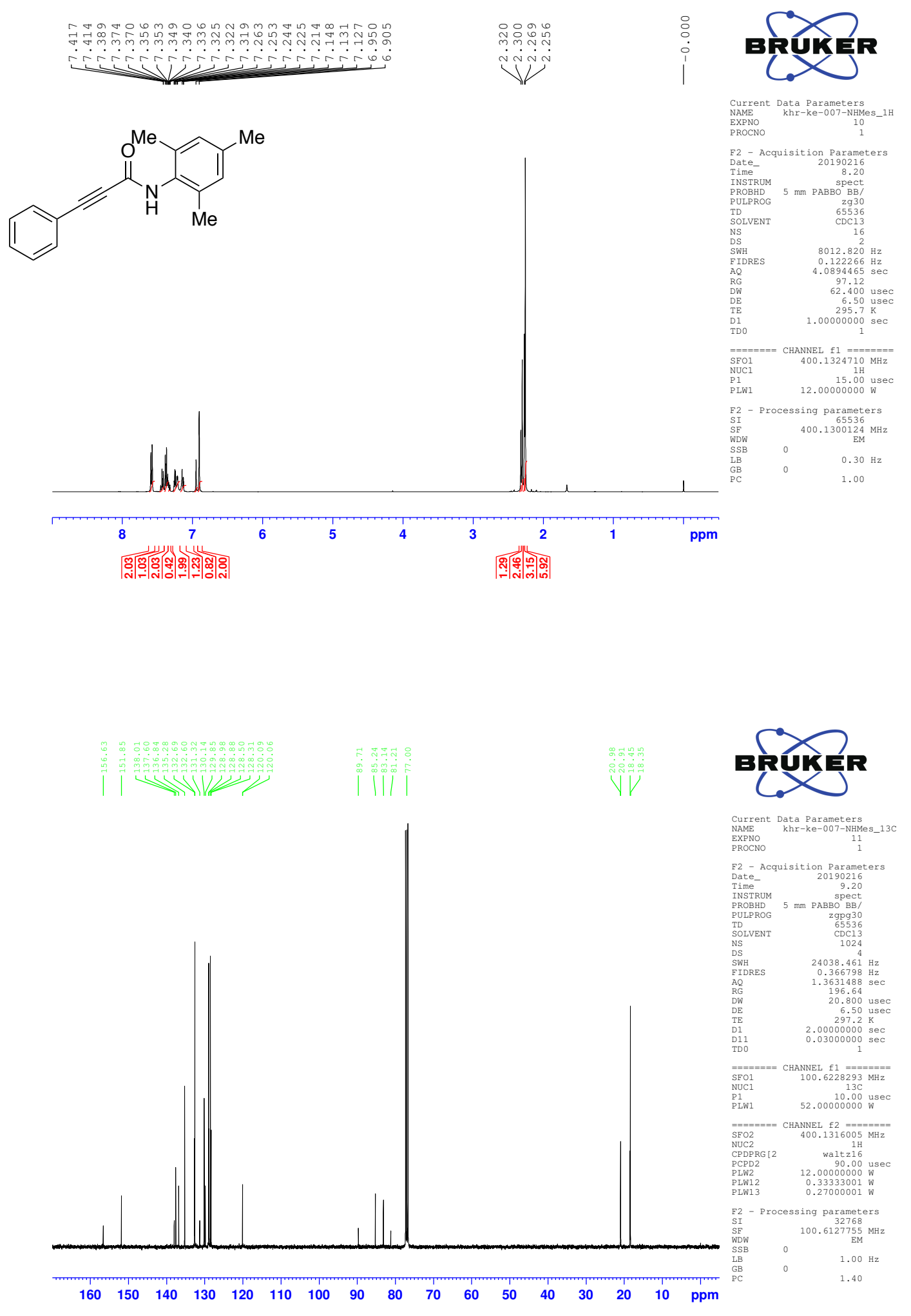
N-ethyl-3-(naphthalen-1-yl)propiolamide (4a)
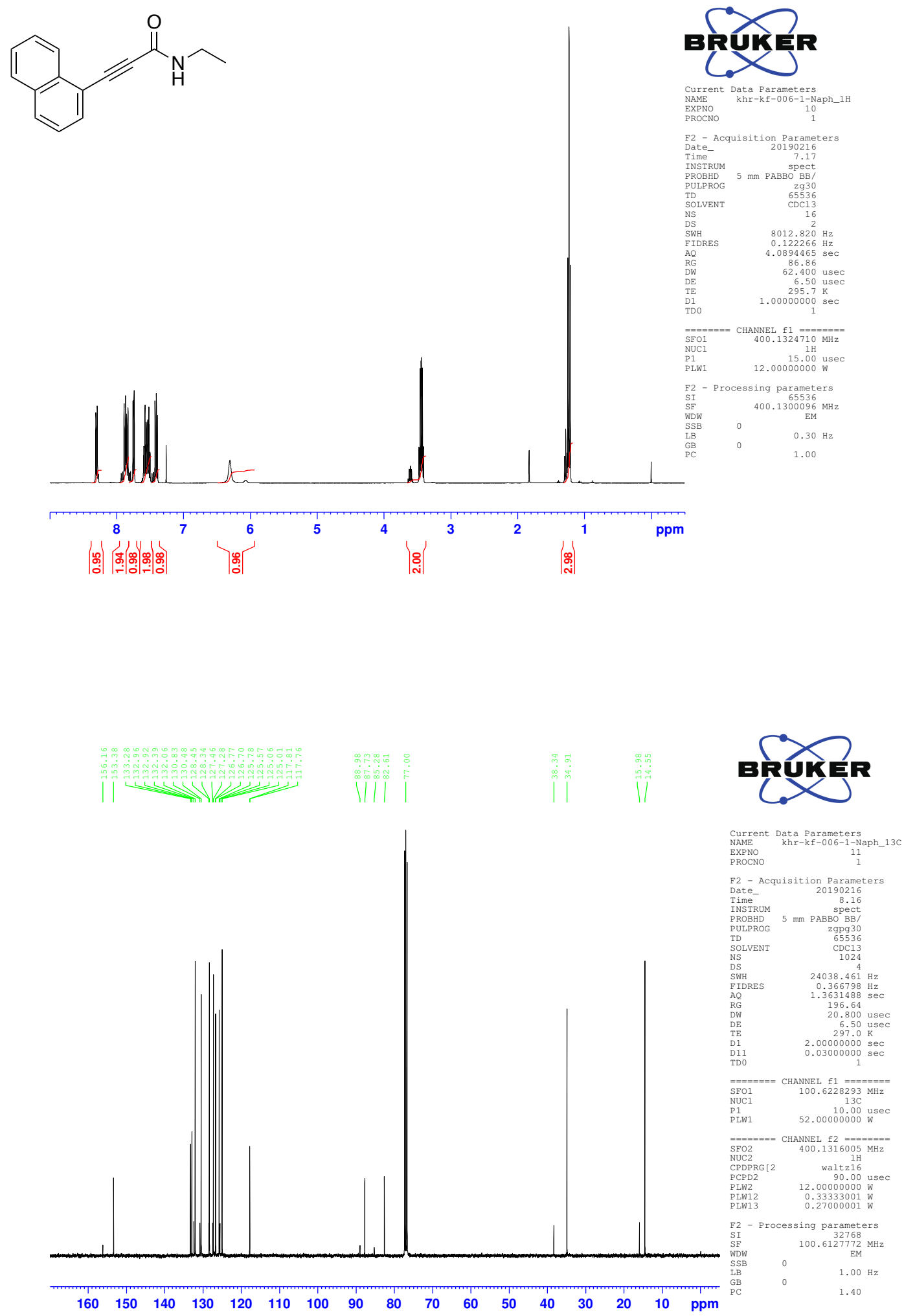

S-77 
$N$-ethyl-3-(naphthalen-2-yl)propiolamide (4b)
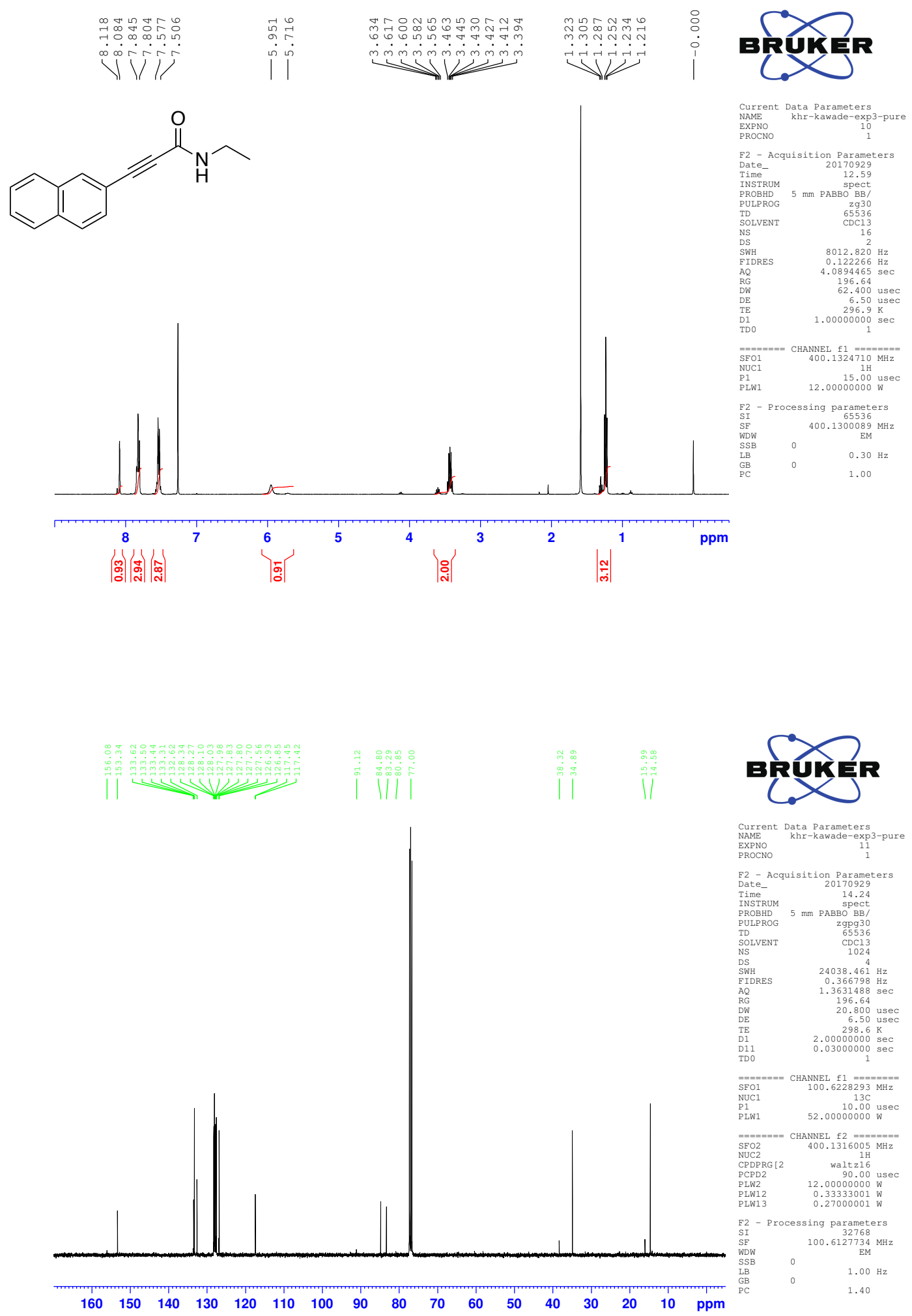
3-(benzo $[d][1,3]$ dioxol-5-yl)- $N$-ethylpropiolamide (4c)
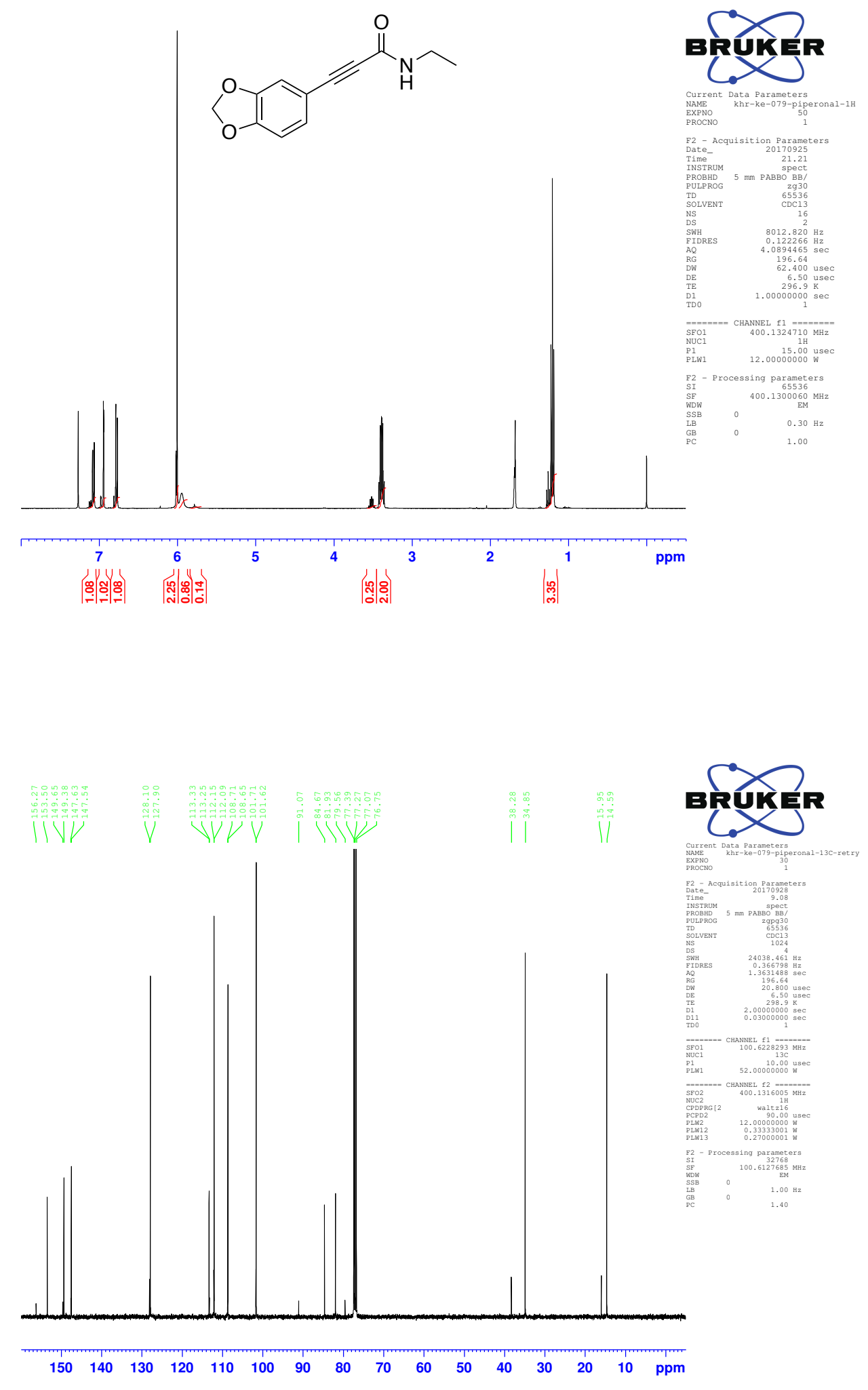
N-ethyl-3-(4-(methylthio)phenyl)propiolamide (4e)

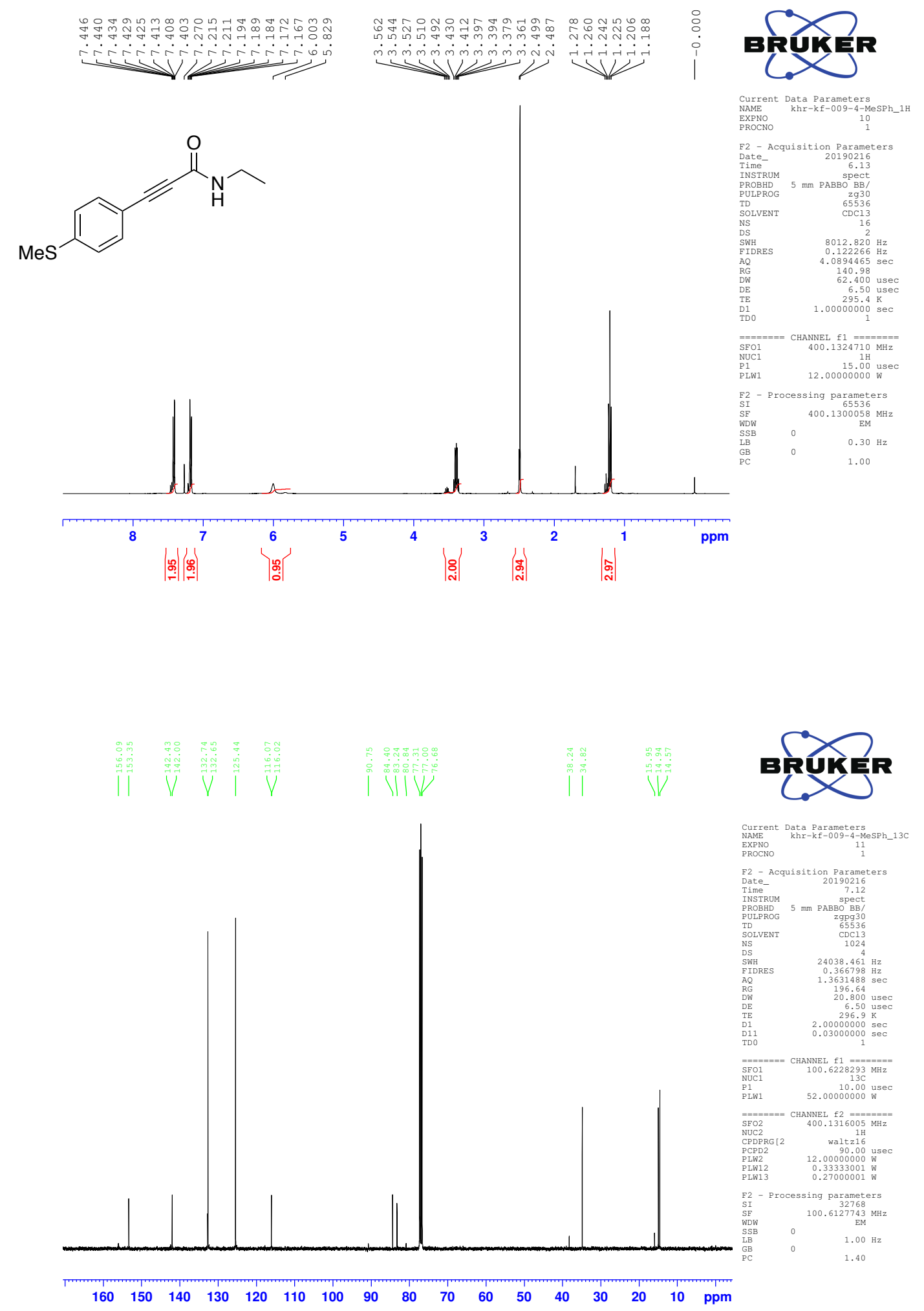


3-(4-(diphenylamino)phenyl)-N-ethylpropiolamide (4f)
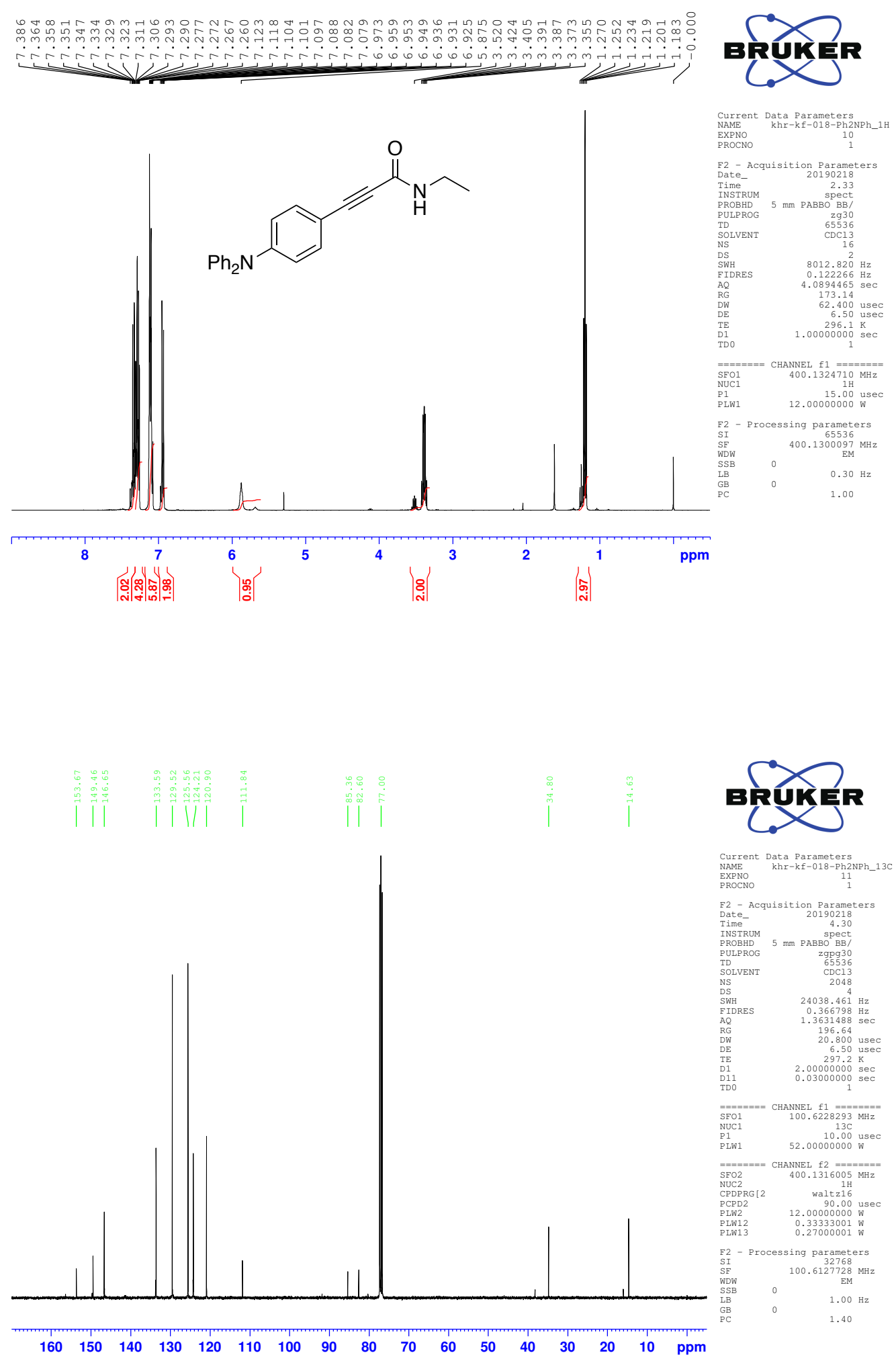
3-(4-(9H-carbazol-9-yl)phenyl)-N-ethylpropiolamide (4g)
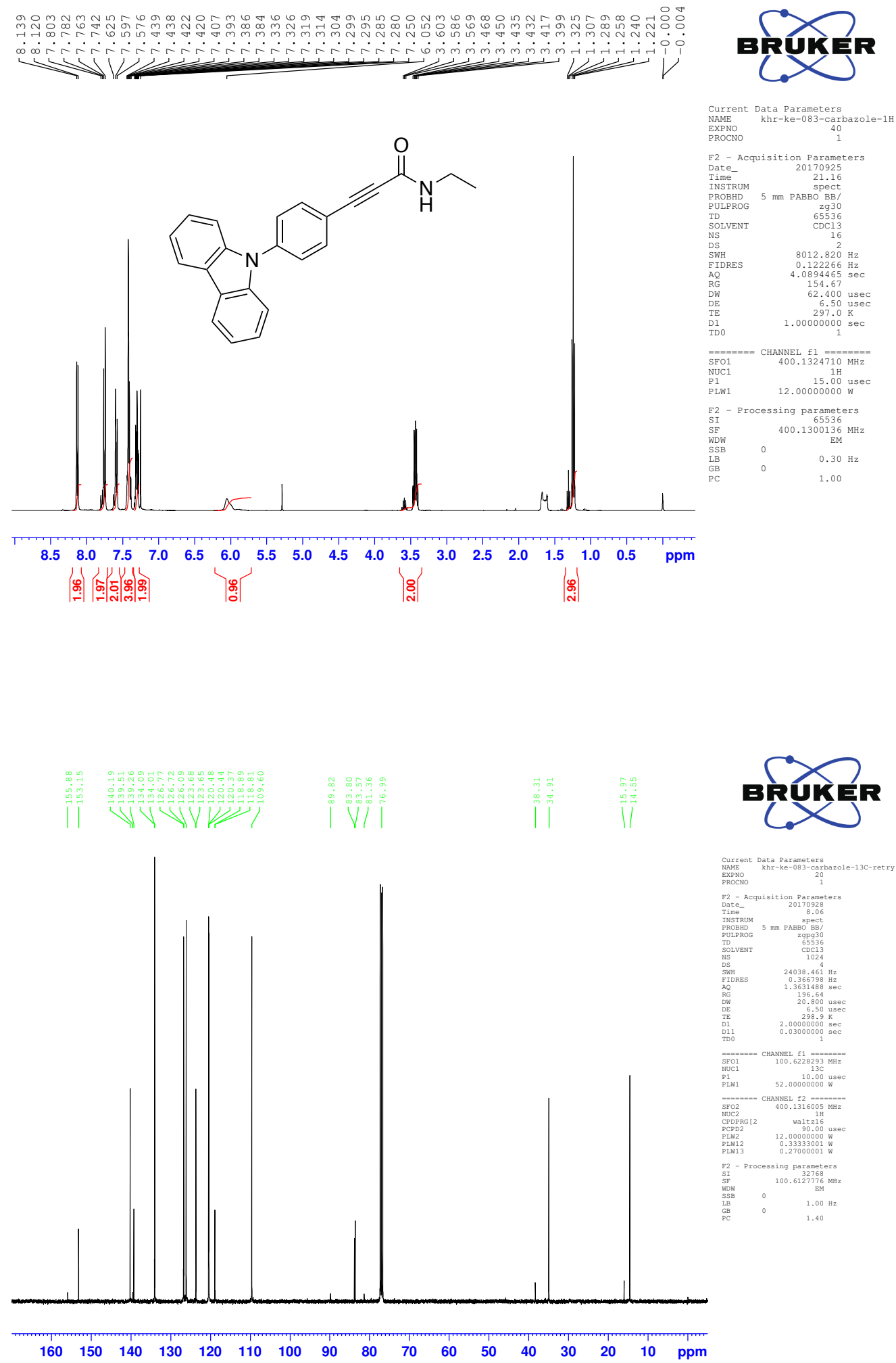

S-82 
3-(4-chlorophenyl)- $\mathrm{N}$-ethylpropiolamide (4h)
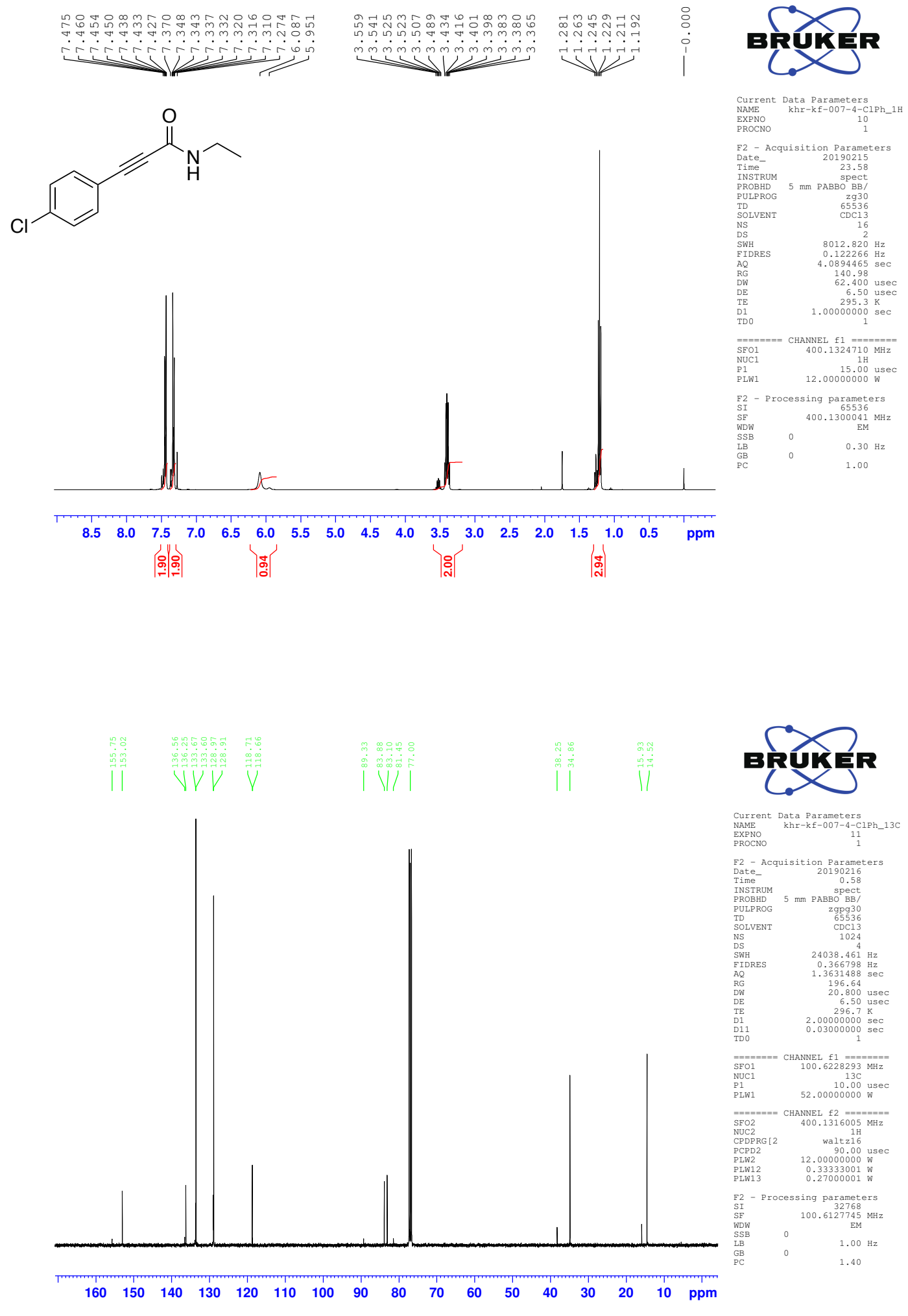
N-ethyl-3-(4-(trifluoromethyl)phenyl)propiolamide (4i)
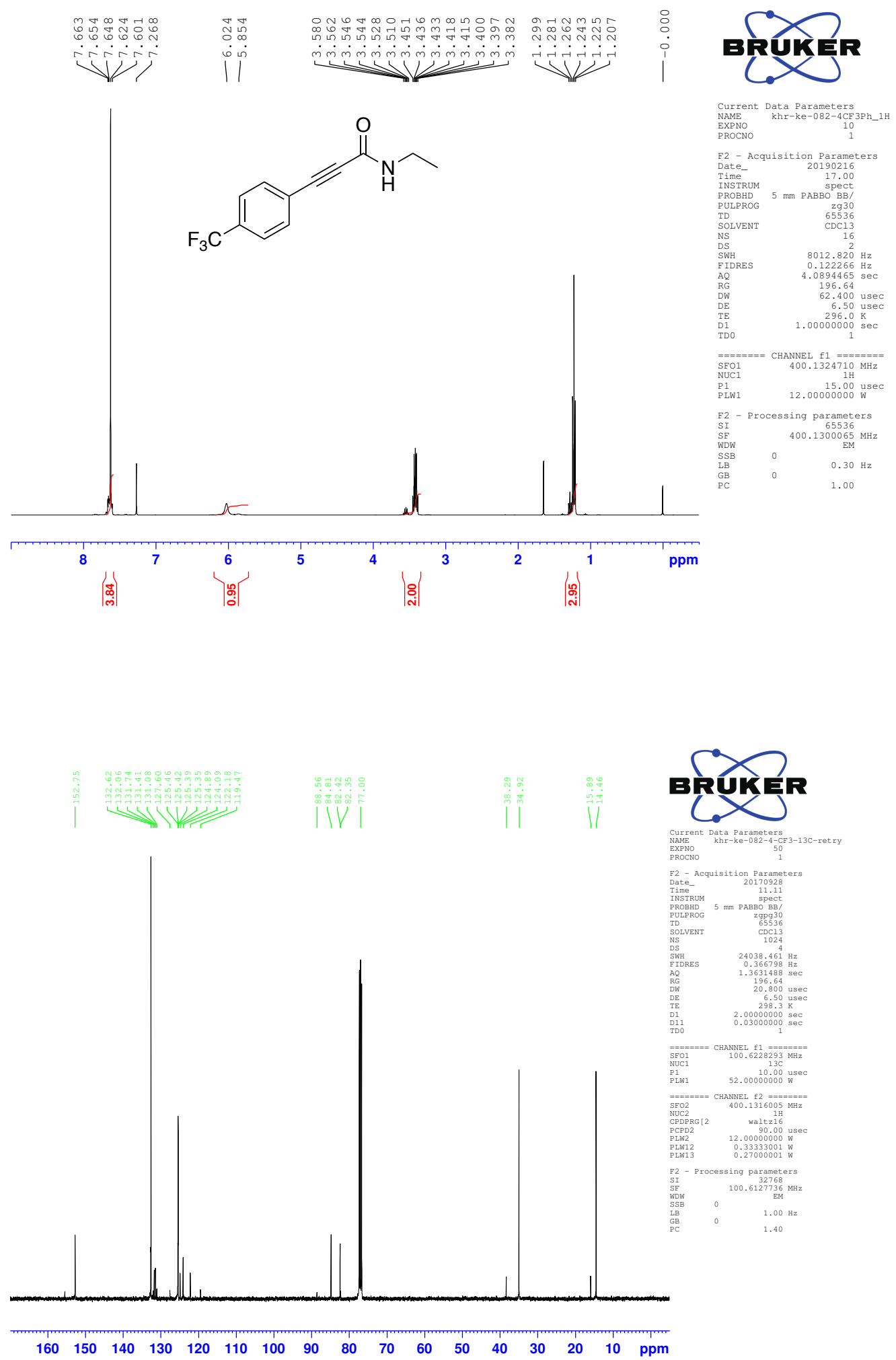

S-84 
ethyl 2-(3-(ethylamino)-3-oxoprop-1-yn-1-yl)benzoate (4j)
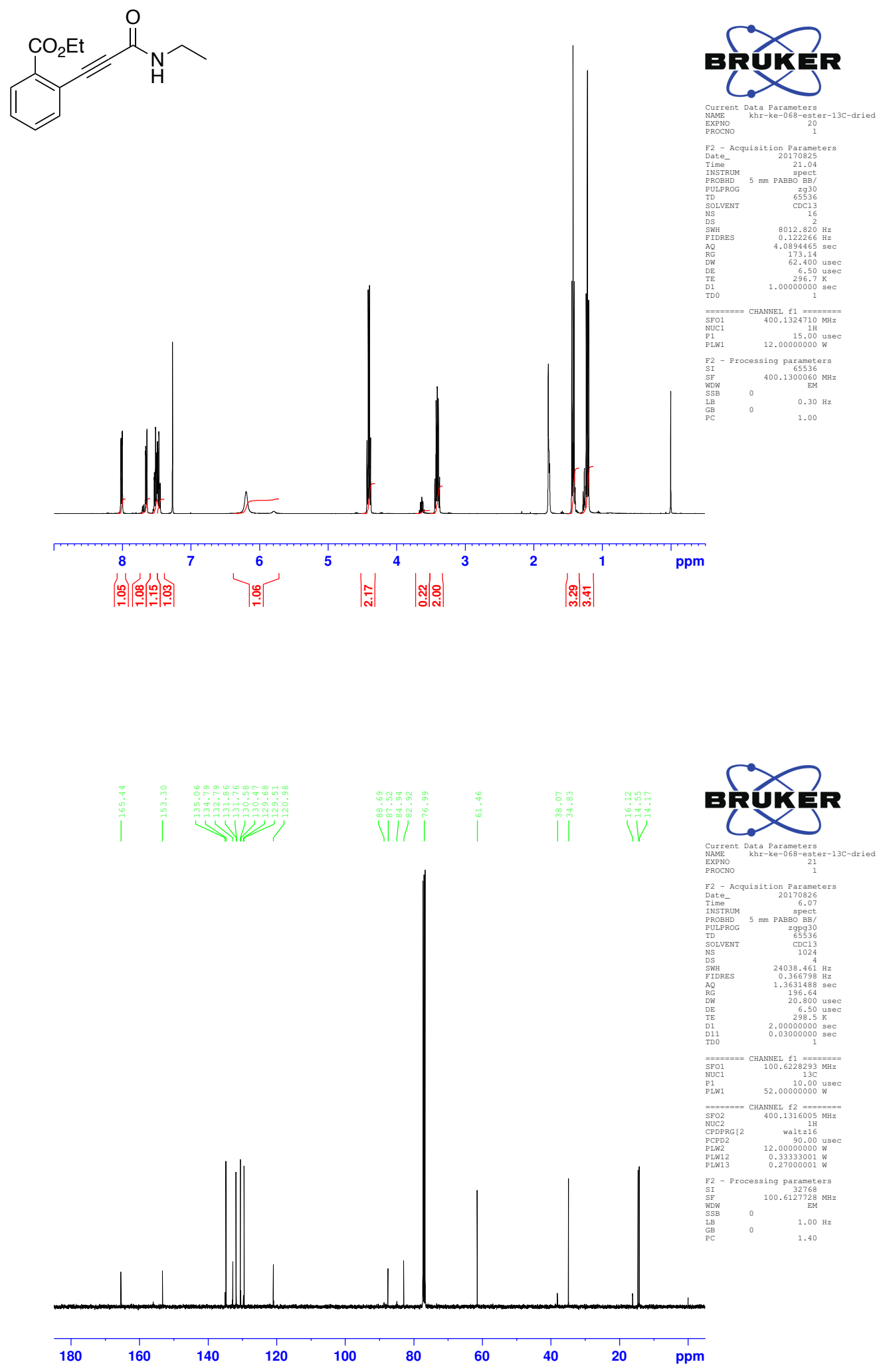

S-85 
3-(4-cyanophenyl)- $N$-ethylpropiolamide (4k)
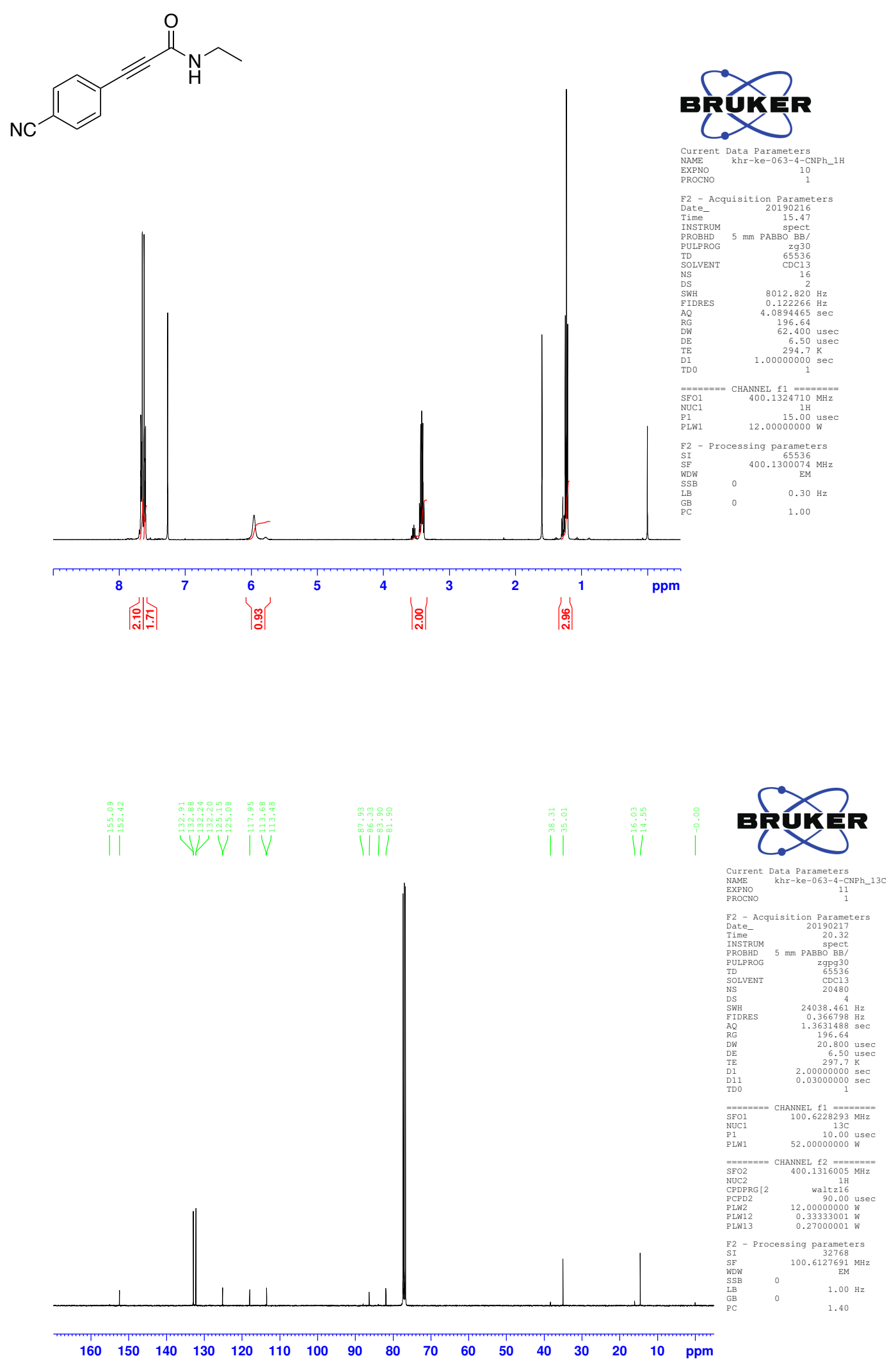
N-ethyl-3-(4-nitrophenyl)propiolamide (41)
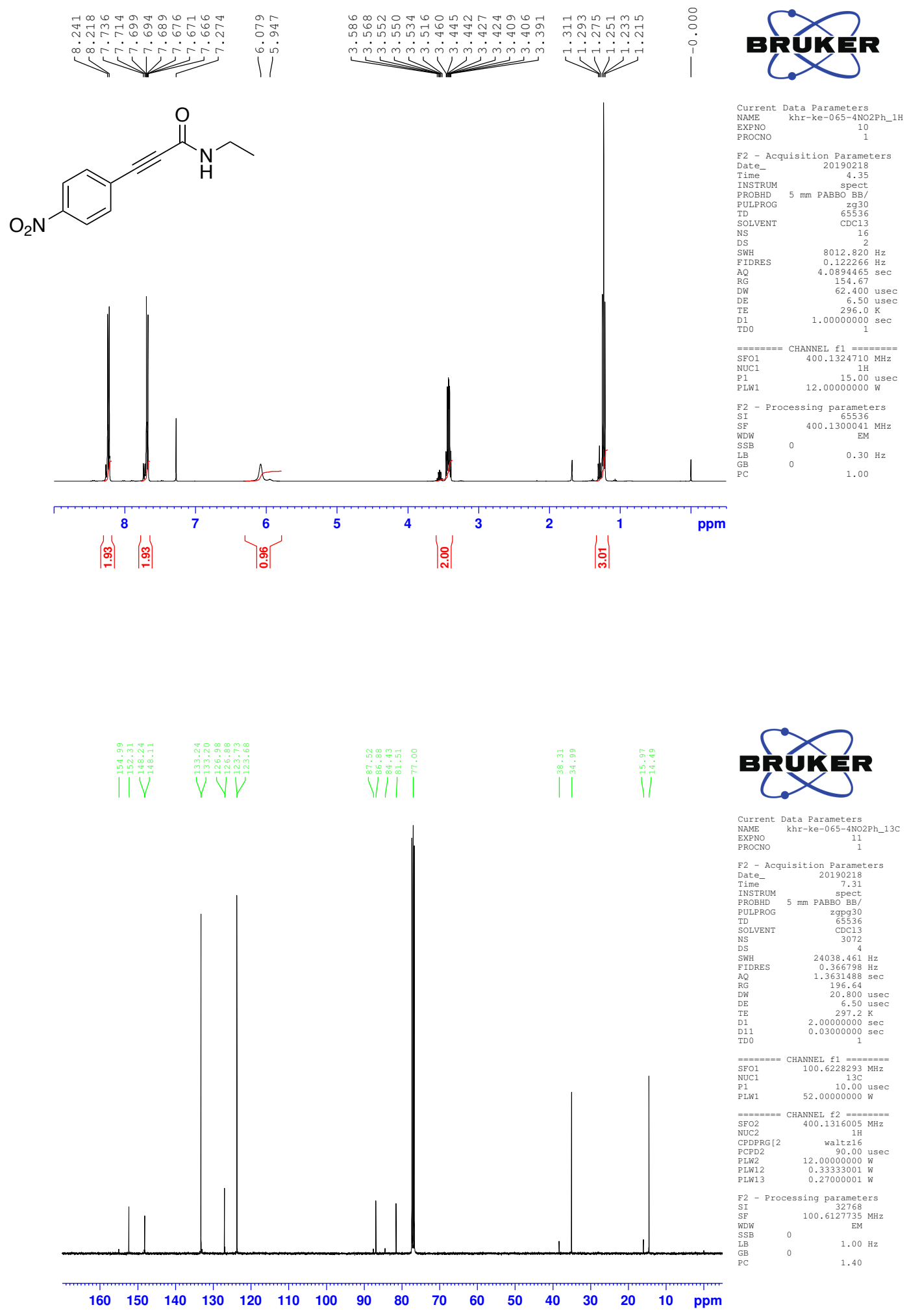
N-ethyl-3-mesitylpropiolamide (4m)
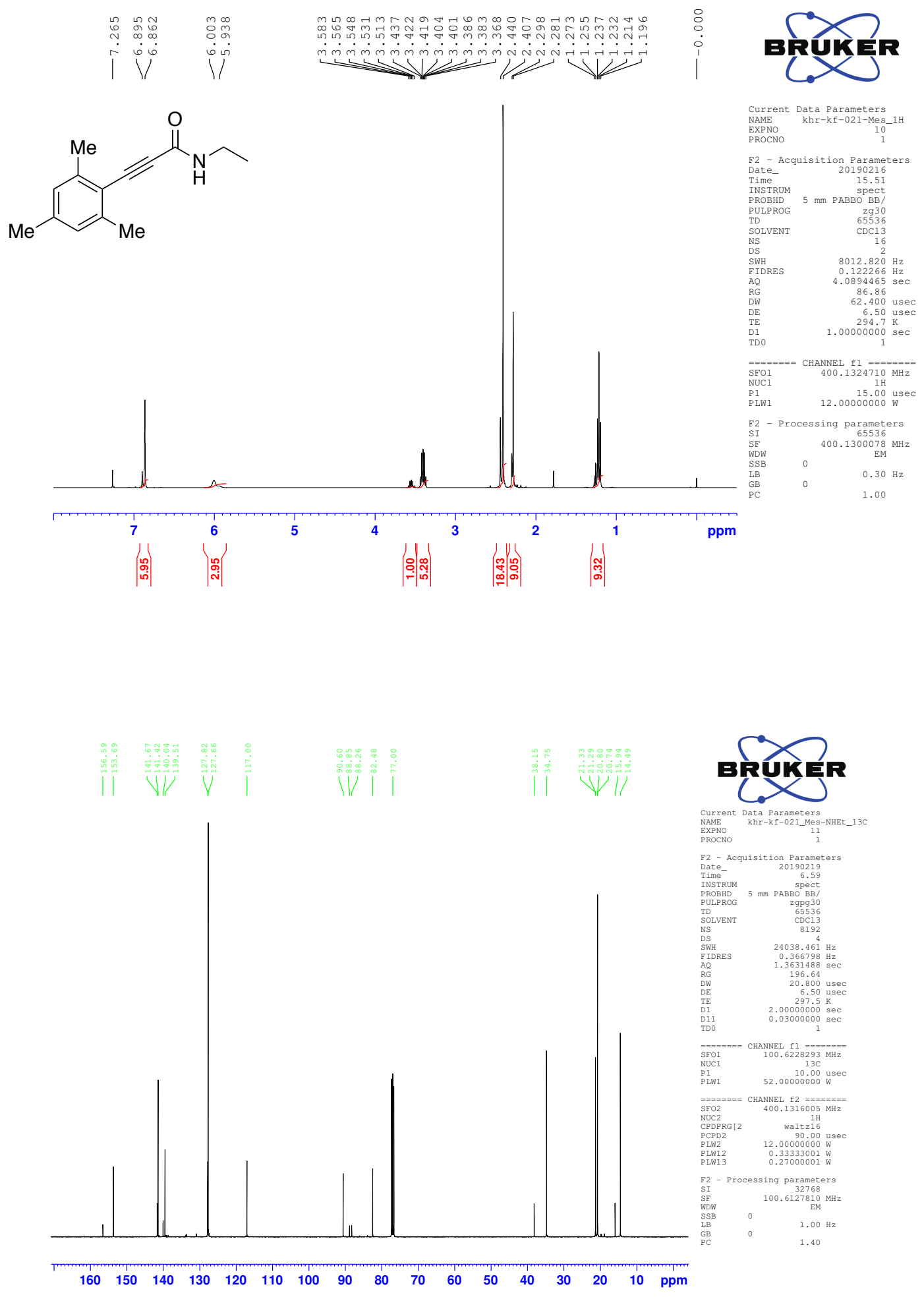

S-88 
N-ethyl-3-(thiophen-3-yl)propiolamide (4n)
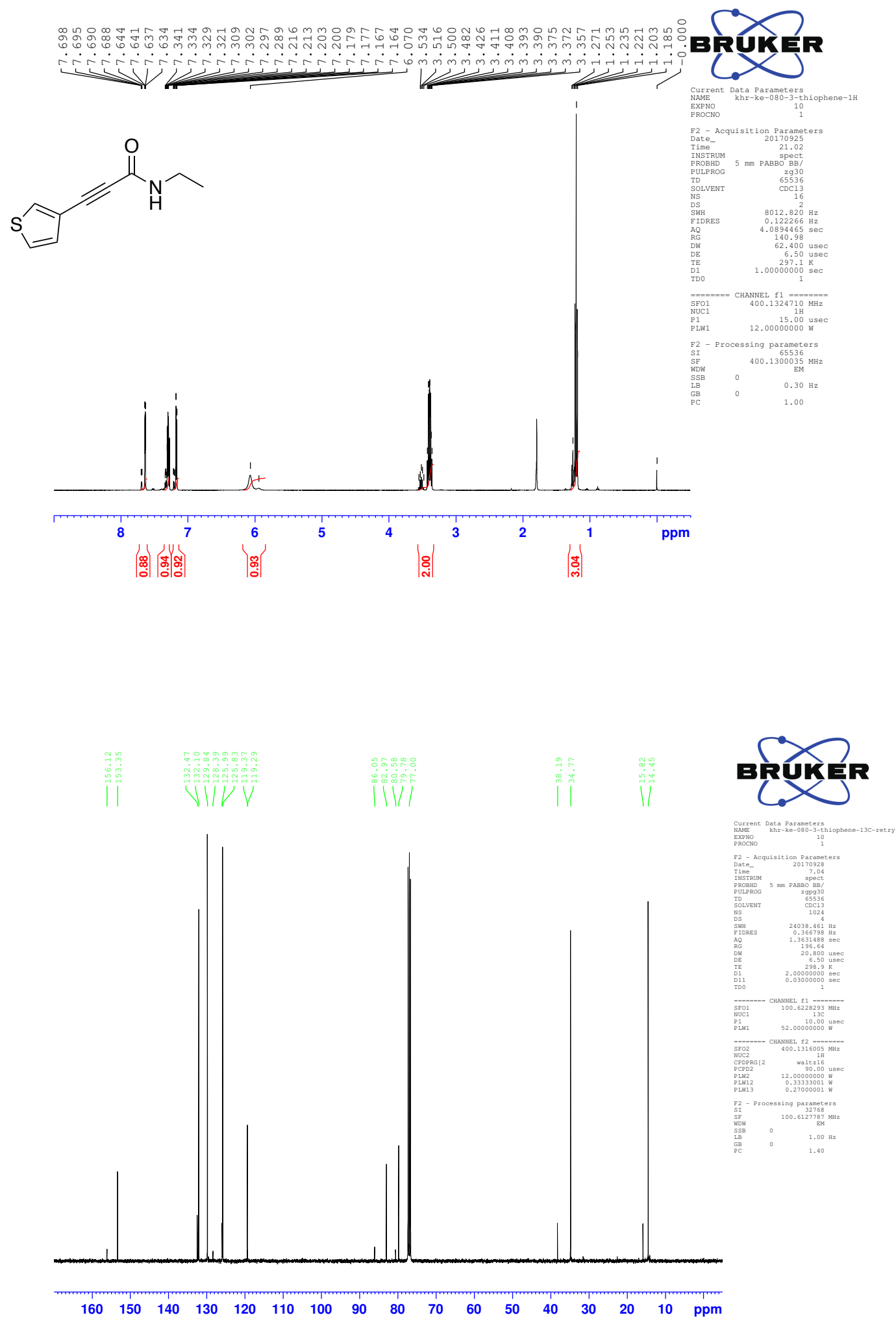

S-89 
3-(benzo $[b]$ thiophen-2-yl)- $N$-ethylpropiolamide (4o)

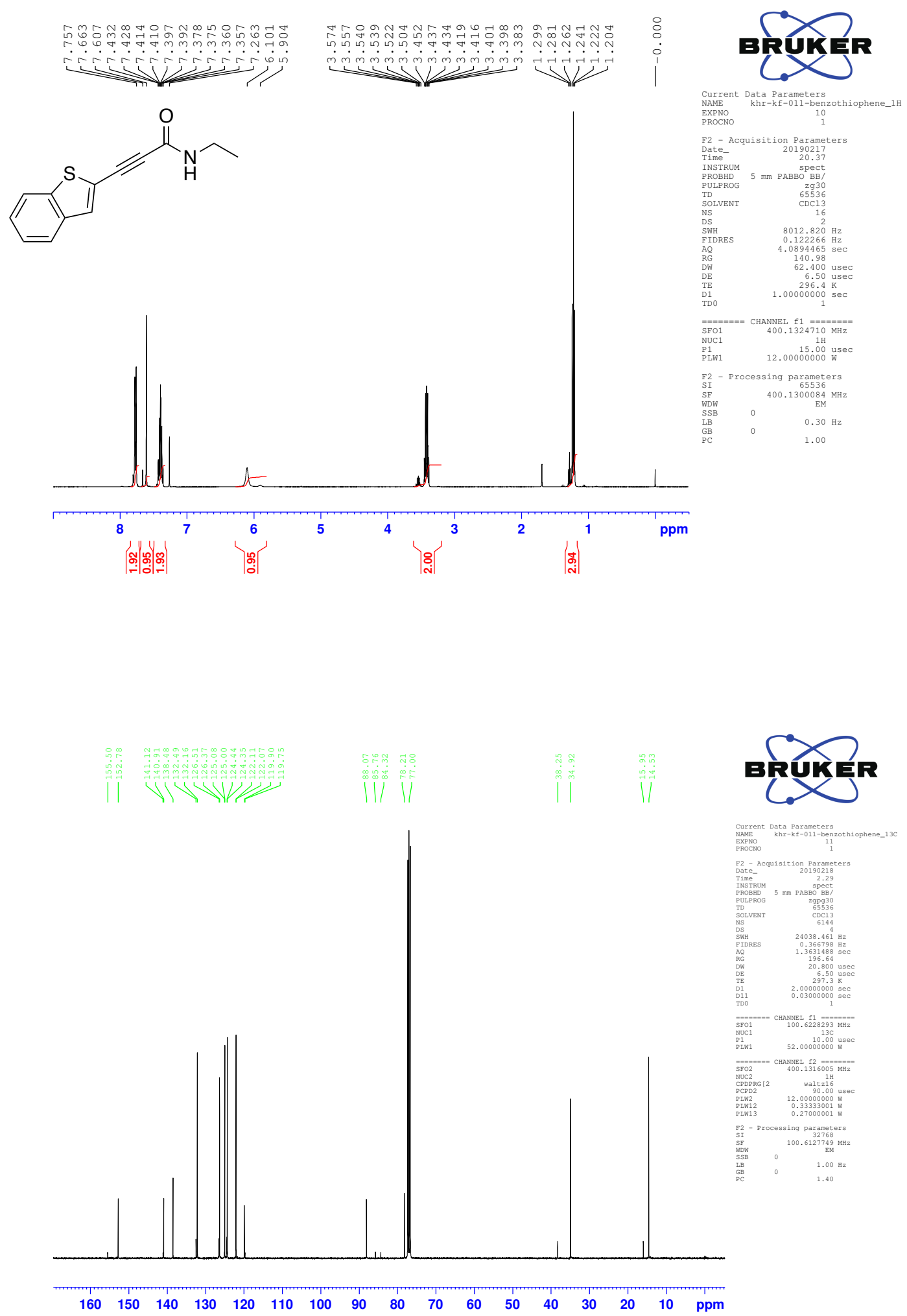


3-(dibenzo $[b, d]$ thiophen-2-yl)- $N$-ethylpropiolamide (4p)

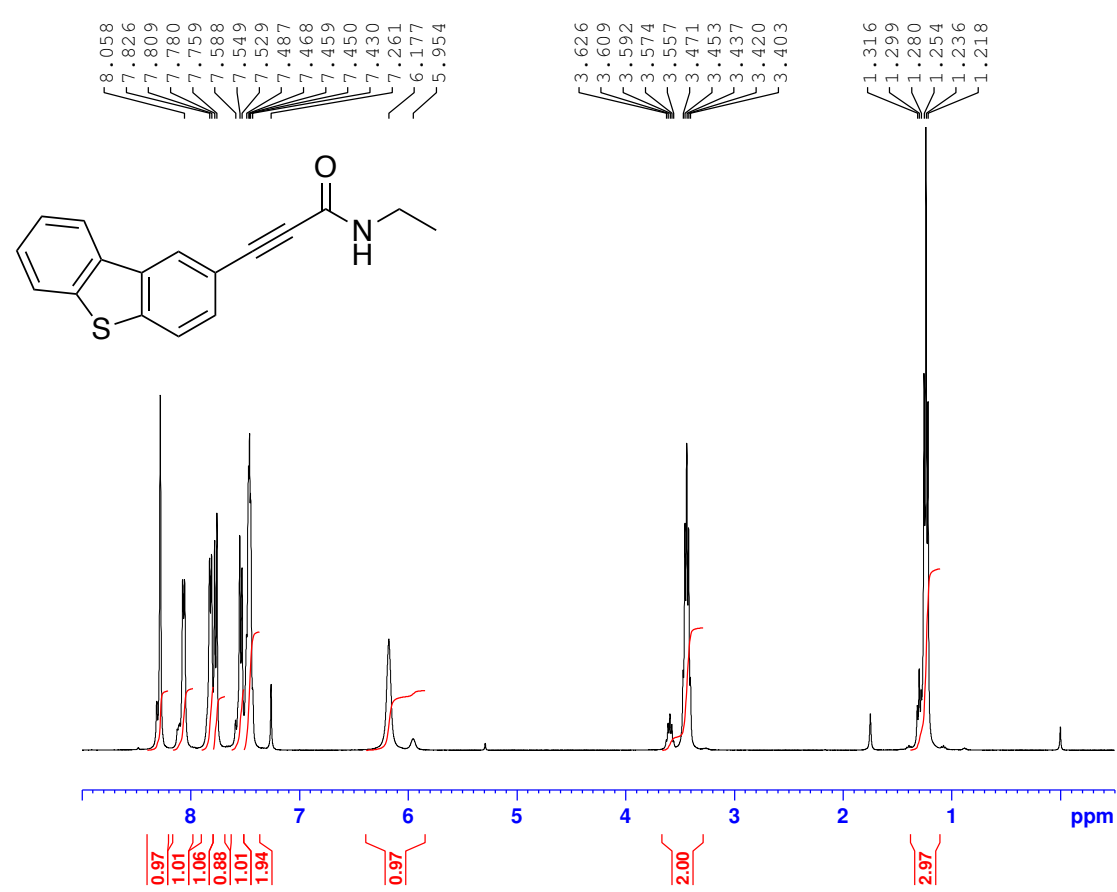

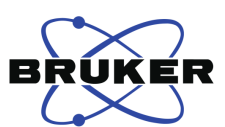

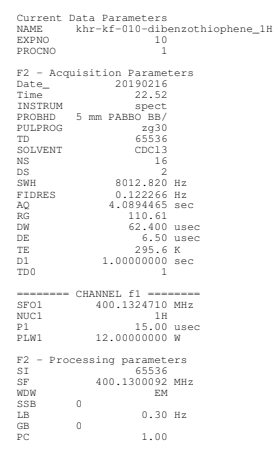

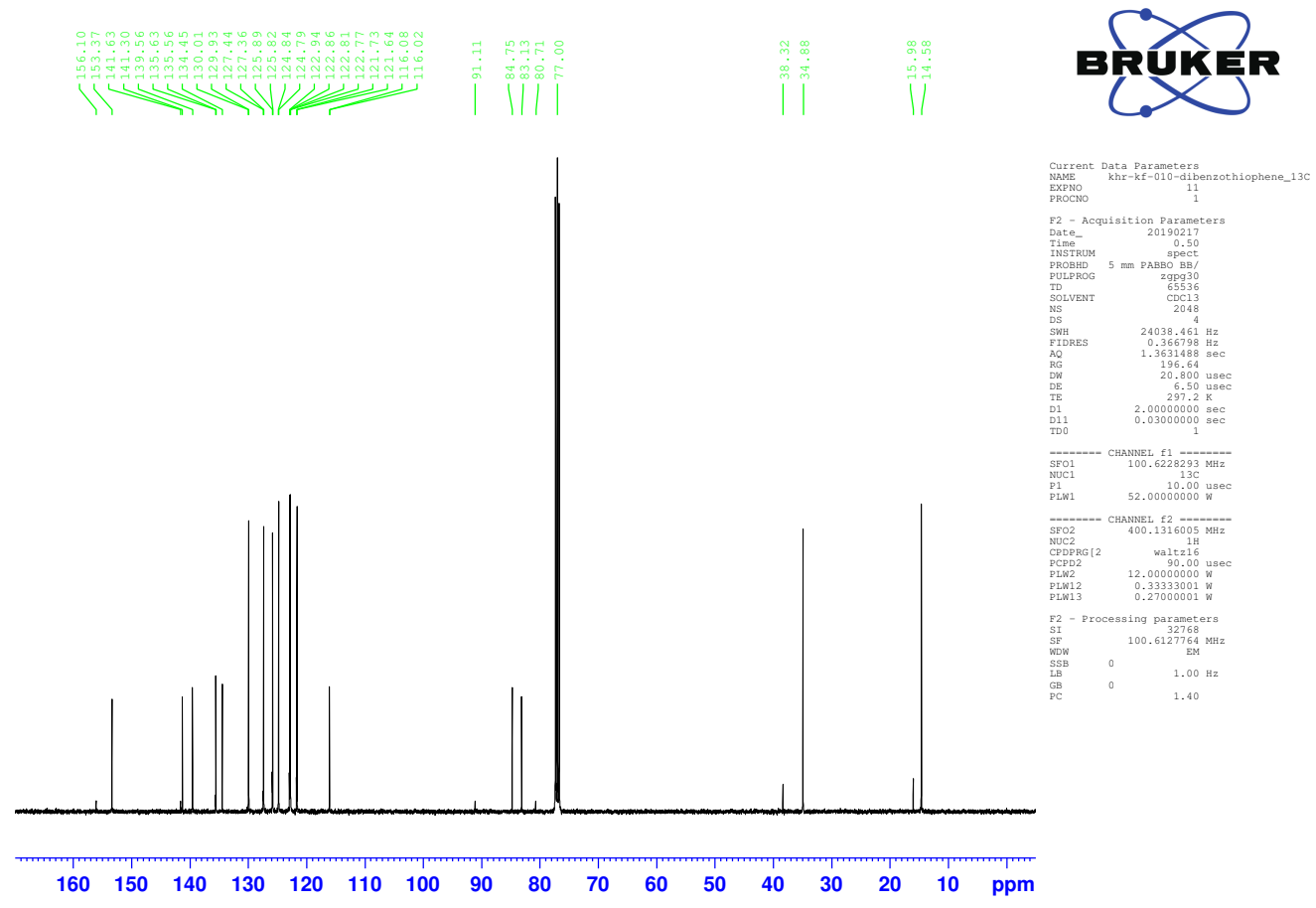

S-91 
3-(cyclohex-1-en-1-yl)- $N$-ethylpropiolamide (4r)
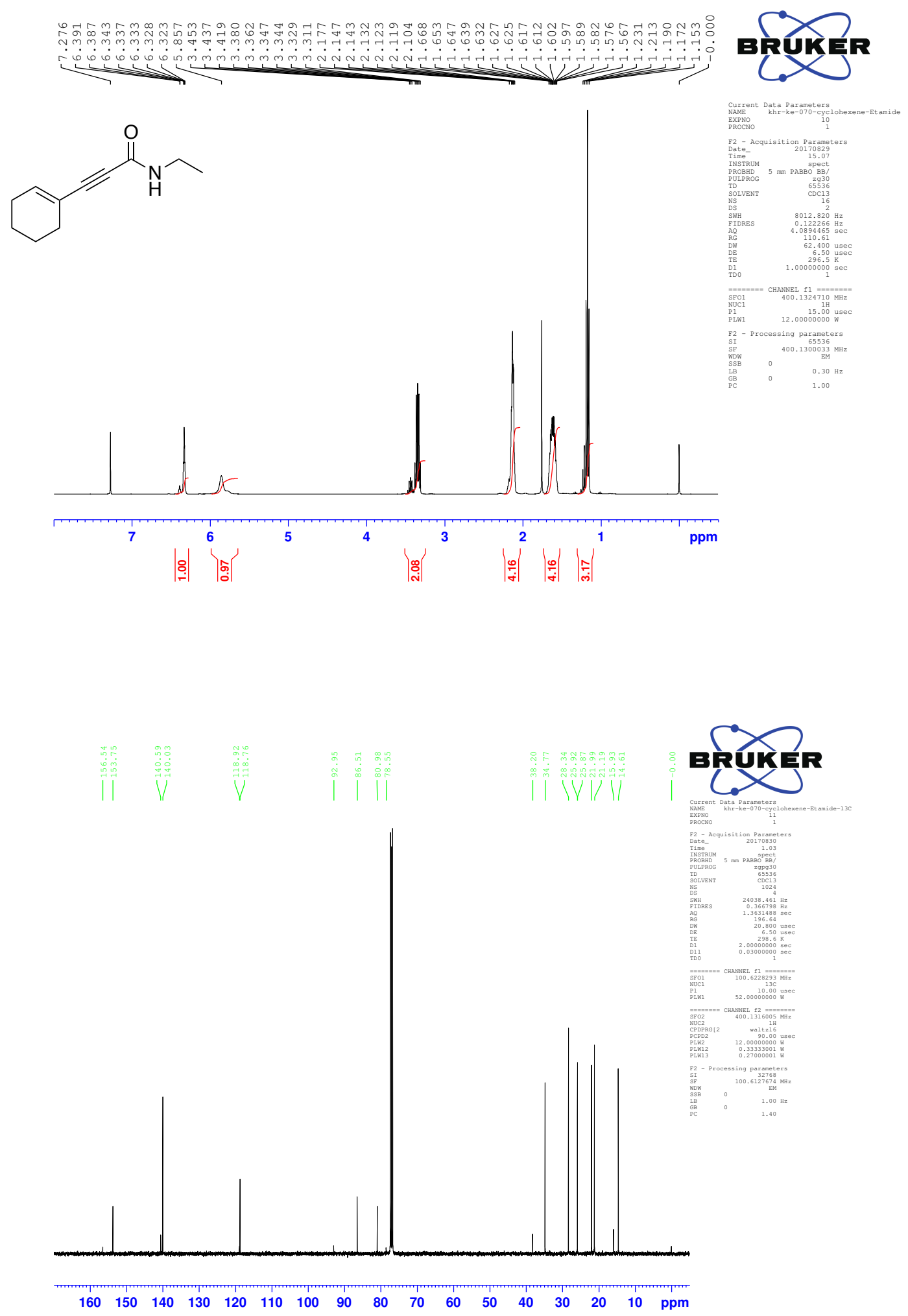

S-92 
(E)-N-ethyl-5-(4-(trifluoromethyl)phenyl)pent-4-en-2-ynamide (3s)
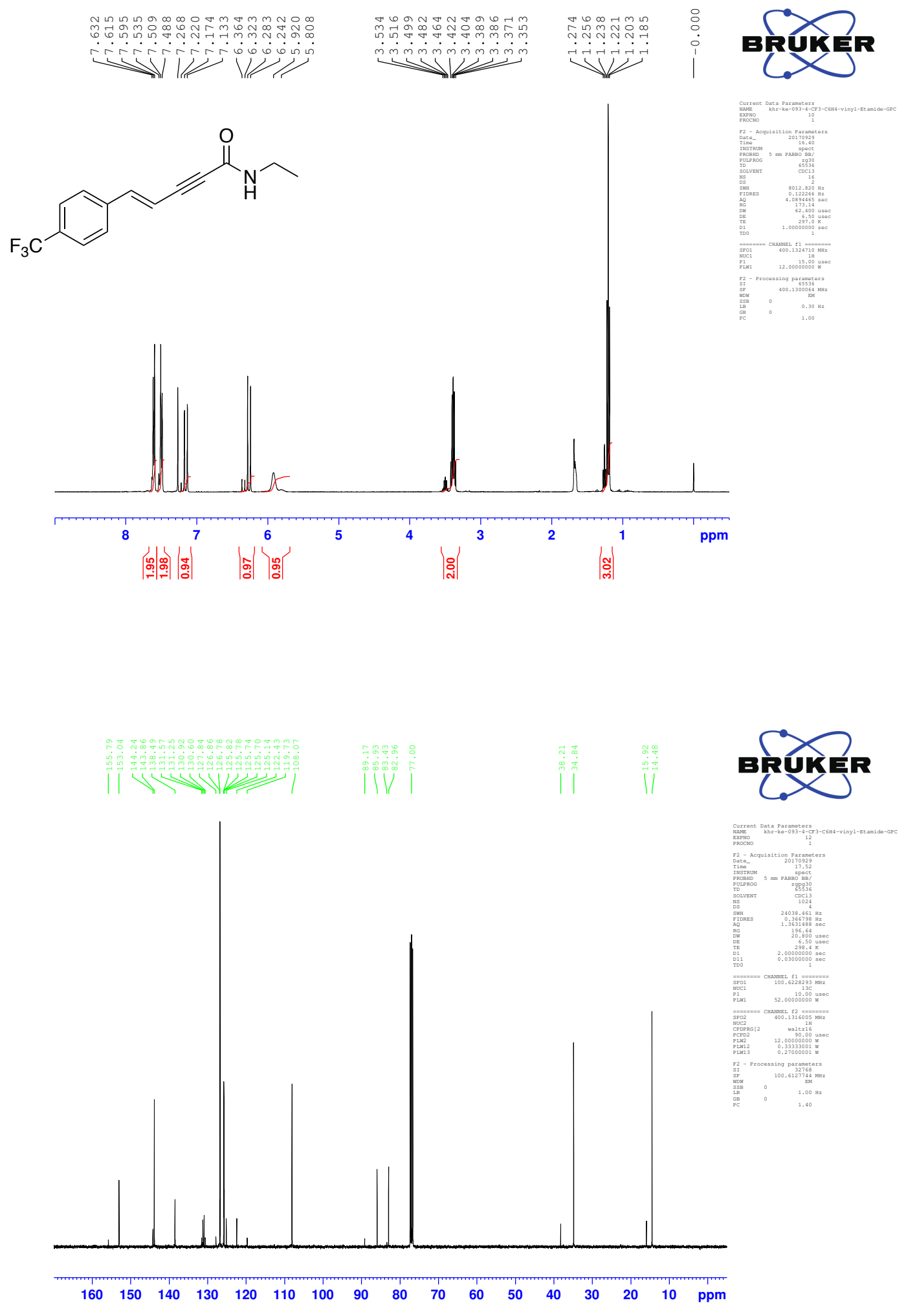
$\mathrm{N}$-ethyl-3-(triisopropylsilyl)propiolamide (4v)
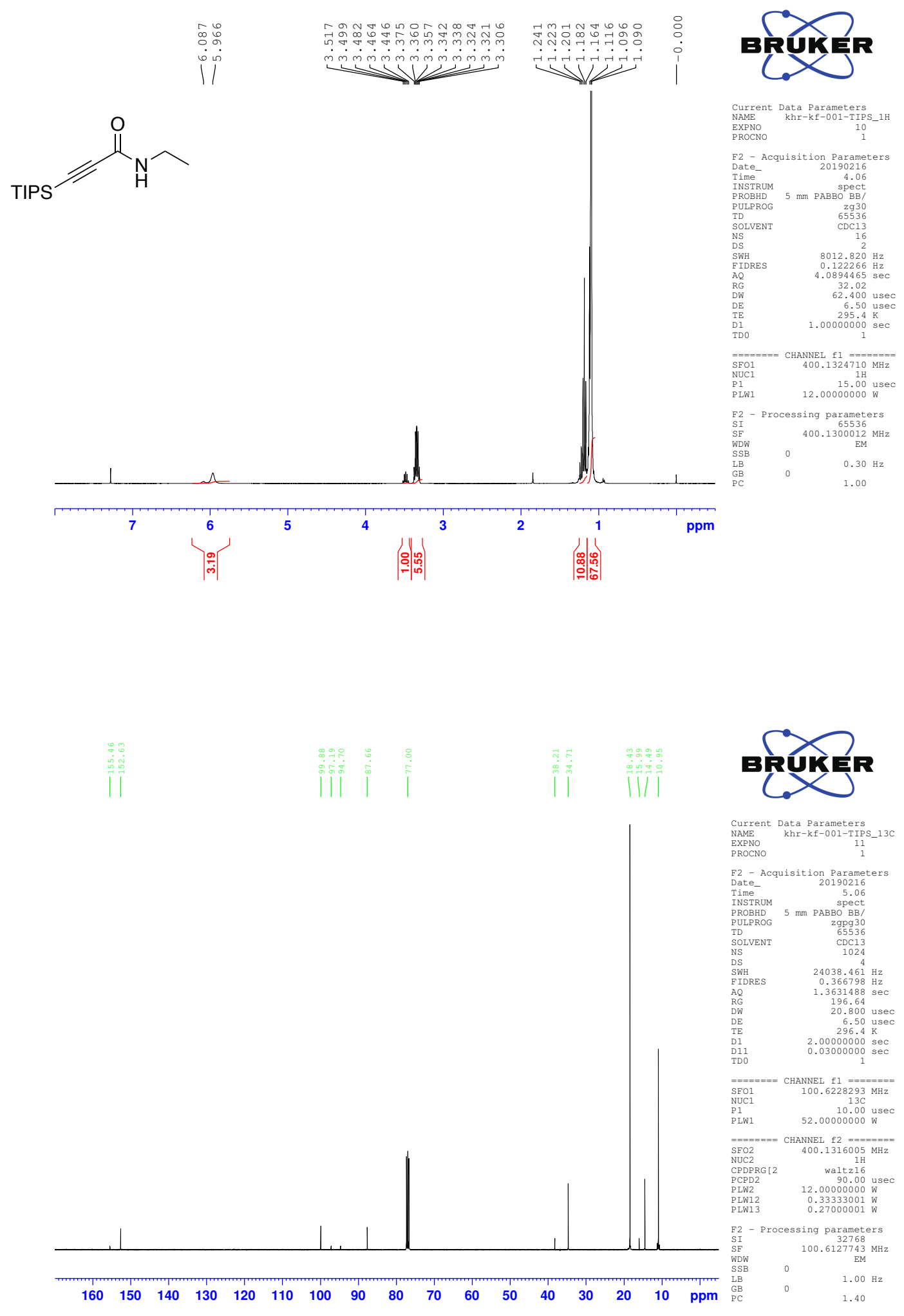